\title{
Finite symplectic matrix groups
}

Von der Fakultät für Mathematik, Informatik und Naturwissenschaften der RWTH Aachen University zur Erlangung des akademischen Grades eines Doktors der Naturwissenschaften genehmigte Dissertation

vorgelegt von

Diplom-Mathematiker

Markus Kirschmer

aus Giengen

Berichter: Universitätsprofessorin Dr. Gabriele Nebe Universitätsprofessor Dr. Wilhelm Plesken

Tag der mündlichen Prüfung: 24. März 2009

Diese Dissertation ist auf den Internetseiten der Hochschulbibliothek online verfügbar. 



\section{Contents}

$\begin{array}{lll}1 & \text { Introduction } & 5\end{array}$

$\begin{array}{lll}2 & \text { Methods } & 9\end{array}$

2.1 Definitions . . . . . . . . . . . . . . . . . . . . . . . . . . . . . . . . . . . . . . . 9

$2.1 .1 \quad$ Symplectic matrix groups . . . . . . . . . . . . . . 99 9

$2.1 .2 \quad$ Primitivity $\ldots \ldots \ldots \ldots \ldots \ldots$

$2.1 .3 \quad$ Generalized Bravais groups . . . . . . . . . . . . . . . . . 18

$2.1 .4 \quad$ General outline of the classification . . . . . . . . . . . . . . . . 20

2.2 Methods . . . . . . . . . . . . . . . . . . . . . . 21

$2.2 .1 \quad$ Normal subgroups of index $2^{k} \ldots \ldots \ldots$. . . . . . . . . 21

$2.2 .2 \quad$ Primitive, normalized and normal critical lattices . . . . . . . . 22

2.2.3 Fields as endomorphism rings (m-parameter argument) . . . . . 25

2.2 .4 Quaternion algebras as endomorphism rings . . . . . . . . . 28

2.3 Algorithms . . . . . . . . . . . . . . . . . . . . . . . . 31

2.4 Some notation $\ldots \ldots \ldots \ldots$

2.5 Some tables . . . . . . . . . . . . . . . . . . . . . . . . . . 37

$2.5 .1 \quad$ Candidates for the layer $\ldots \ldots \ldots$. . . . . . . . . . 37

2.5.2 Candidates for the Fitting subgroup. . . . . . . . . . . . . . . . 39

2.5 .3 Tables for number fields . . . . . . . . . . . . . . . . . . . 39

3 Some infinite s.i.m.f. families 43

$3.1 \quad$ Some subgroups of $\mathrm{Sp}_{p-1}(\mathbb{Q})$. . . . . . . . . . . . . . . . . . . . . . . . . . . 43

$3.2 \quad$ Some subgroups of $\mathrm{Sp}_{p+1}(\mathbb{Q})$

3.3 The group $Q D_{2^{n}} \quad \ldots \ldots \ldots \ldots \ldots$. . . . . . . . . . . . . . . . . . . 45

3.4 The group $2_{+}^{1+2 n} \ldots \ldots \ldots \ldots \ldots$. . . . . . . . . . . . . . . . . . . . . . . 45

3.5 The group $p_{+}^{1+2 n} \ldots \ldots \ldots \ldots \ldots$. . . . . . . . . . . . . . . . . . . 47

\begin{tabular}{lll}
\hline 4 & The classification & 49
\end{tabular}

4.1 Dimension $2 \mathrm{p} \ldots \ldots \ldots \ldots \ldots$

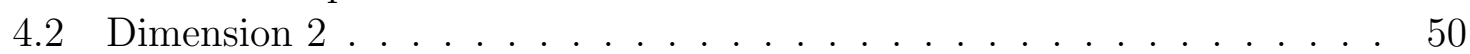

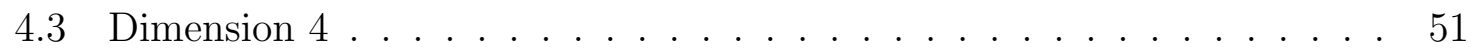

$4.3 .1 \quad$ Irreducible cyclic subgroups . . . . . . . . . . . . . . . . . 51

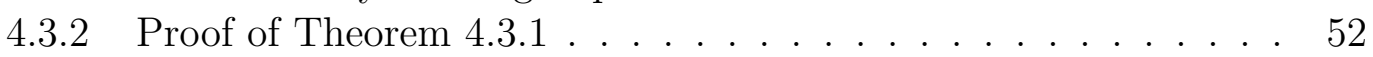

4.4 Dimension 6 $\ldots \ldots \ldots \ldots \ldots$

4.5 Dimension $8 \ldots \ldots \ldots$

$4.5 .1 \quad$ Irreducible cyclic subgroups . . . . . . . . . . . . . . . . . 55

$4.5 .2 \quad$ Proof of Theorem $4.5 .1 \ldots \ldots \ldots$. . . . . . . . . . . . . 57 
4.6 Dimension 10 . . . . . . . . . . . . . . . . . . . . . . . . . . . . . . . . . . . 64

4.7 Dimension $12 \ldots \ldots \ldots \ldots$

$4.7 .1 \quad$ Irreducible cyclic subgroups . . . . . . . . . . . . . . . 66

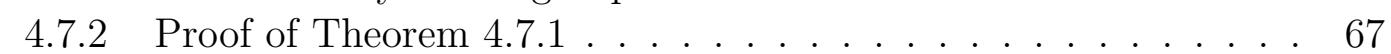

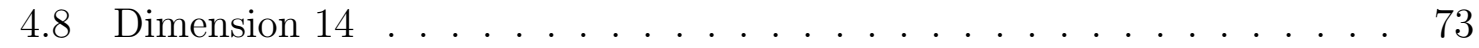

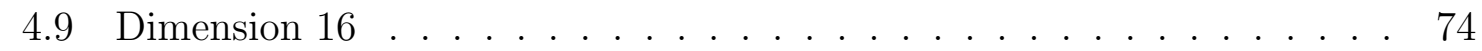

$4.9 .1 \quad$ Irreducible cyclic subgroups . . . . . . . . . . . . . . . . . . . . . 81

4.9.2 The case $O_{17}(G)=1$ and $O_{5}(G) \neq 1 \quad \ldots \ldots$. . . . . . . 87

4.9 .3 The case $O_{17}(G)=O_{5}(G)=1$ and $O_{3}(G) \neq 1$. . . . . . . . . . 92

4.9 .4 The case $O_{p}(G)=1$ for all odd primes $p$. . . . . . . . . . . 98

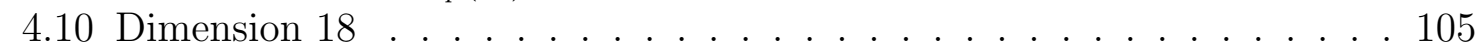

4.11 Dimension 20 . . . . . . . . . . . . . . . . . . . . . . . . . . . . . . . . . . . . . . . . . . 108

4.11 .1 Irreducible cyclic subgroups . . . . . . . . . . . . . . . . . 110

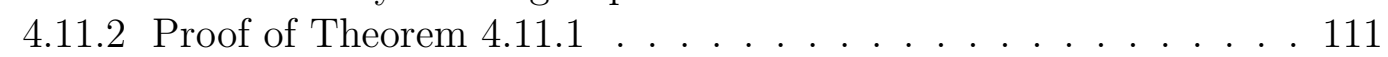

4.12 Dimension $22 \ldots \ldots \ldots \ldots$

\begin{tabular}{|rr}
\hline A Invariant Forms & 119
\end{tabular}

A.1 Dimension 2 . . . . . . . . . . . . . . . . . . . . . . . . . . . . . . . . . . . . . 120

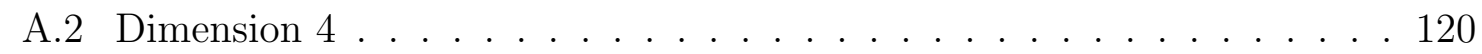

A.3 Dimension 6 . . . . . . . . . . . . . . . . . . . . . . . . . . . . 120

A.4 Dimension 8 . . . . . . . . . . . . . . . . . . . . . . . . . . 121

A.5 Dimension 10 . . . . . . . . . . . . . . . . . . . . . . . . . . . . . . . . . . . . 122

A.6 Dimension 12 . . . . . . . . . . . . . . . . . . . . . . . . . . . . . . . . . . . 122

A.7 Dimension 14 . . . . . . . . . . . . . . . . . . . . . . 125

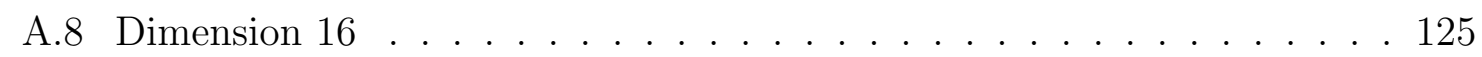

A.9 Dimension 18 . . . . . . . . . . . . . . . . . . . . . . . . . . . . 135

A.10 Dimension 20 . . . . . . . . . . . . . . . . . . . . . 136

A.11 Dimension 22 . . . . . . . . . . . . . . . . . . . . . . . . . . 142

\begin{tabular}{ll}
\hline Nomenclature & 145
\end{tabular}

\begin{tabular}{ll}
\hline References & 147
\end{tabular} 


\section{Chapter 1}

\section{Introduction}

The finite subgroups of $\mathrm{GL}_{m}(\mathbb{Q})$ are those subgroups that fix a full lattice in $\mathbb{Q}^{1 \times m}$ together with some positive definite symmetric form (see Chapter 2 for precise definitions).

A subgroup of $\mathrm{GL}_{m}(\mathbb{Q})$ is called symplectic, if it fixes a nondegenerate skewsymmetric form. Such groups only exist if $m$ is even. A symplectic subgroup of $G_{m}(\mathbb{Q})$ is called maximal finite symplectic if it is not contained in another finite symplectic subgroup of $\mathrm{GL}_{m}(\mathbb{Q})$.

This thesis classifies all conjugacy classes of maximal finite symplectic matrix groups in $\mathrm{GL}_{m}(\mathbb{Q})$ for $m \leq 22$.

Such classifications have a long tradition. Minkowski [Min87] gave upper bounds on the orders of finite subgroups of $\mathrm{GL}_{m}(\mathbb{Q})$ using the theory of quadratic forms. Later, Schur [Sch05] gave bounds on the orders of finite subgroups of $\mathrm{GL}_{m}(\mathbb{C})$ having rational traces using character theory. Serre extended this work to arbitrary fields (see [GTT07]). In [Bli17] Blichfeldt classified all finite subgroups of $\mathrm{PGL}_{2}(\mathbb{C})$ and $\mathrm{PGL}_{3}(\mathbb{C})$. The finite (quasiprimitive) subgroups of $\mathrm{GL}_{m}(\mathbb{C})$ for $m \leq 10$ have been determined by Blichfeld, Brauer, Lindsey, Wales and Feit (see [Fei76]). These results do not depend on the classification of all finite simple groups. Using the classification of all finite simple groups, the maximal finite subgroups of $\mathrm{GL}_{m}(\mathbb{Q})$ have been determined in [BBNZ77, Ple91, NP95, Neb95, Neb96] for $m \leq 31$. Similarly, the maximal finite subgroups of $\mathrm{GL}_{m}(\mathcal{Q})$ have been classified in Neb98 for all totally definite quaternion algebras $\mathcal{Q}$ with $m \cdot \operatorname{dim}_{\mathbb{Q}}(\mathcal{Q}) \leq 40$.

Each conjugacy class of symplectic matrix groups contains a representative $G$ in $\operatorname{Sp}_{2 n}(\mathbb{Q})=\left\{g \in \mathrm{GL}_{2 n}(\mathbb{Q}) \mid g J_{n} g^{\text {tr }}=J_{n}\right\}$ where $J_{n}=\left(\begin{array}{rr}0 & I_{n} \\ -I_{n} & 0\end{array}\right)$. So one might ask how the orbit $\left\{G^{x} \mid x \in \mathrm{GL}_{2 n}(\mathbb{Q})\right.$ such that $\left.G^{x}<\operatorname{Sp}_{2 n}(\mathbb{Q})\right\}$ decomposes into $\mathrm{Sp}_{2 n}(\mathbb{Q})$-conjugacy classes. If the commuting algebra of $G$ is a quadratic number field, then Lemma 2.1 .12 shows that there exists a parametrization of these classes using norm groups. In particular, it turns out that each orbit decomposes into infinitely many $\operatorname{Sp}_{2 n}(\mathbb{Q})$-conjugacy classes. Hence only $\mathrm{GL}_{2 n}(\mathbb{Q})$-conjugacy classes of symplectic matrix groups are considered in this thesis.

The natural representation of a maximal finite symplectic matrix group is a sum of pairwise nonisomorphic rationally irreducible representations that yield maximal finite 
symplectic matrix groups. Thus it suffices to classify only the (conjugacy classes of) symplectic irreducible maximal finite (s.i.m.f.) matrix groups.

Each s.i.m.f. group $G<\mathrm{GL}_{2 n}(\mathbb{Q})$ is contained in a rationally irreducible maximal finite (r.i.m.f.) group $H \leq \mathrm{GL}_{2 n}(\mathbb{Q})$. But in practice, this fact cannot be used to classify all s.i.m.f. groups by computing proper subgroups of such groups $H$ since the index $[H: G]$ can be very large. For example, the s.i.m.f. group $Q D_{64}<\mathrm{GL}_{16}(\mathbb{Q})$ is contained in the r.i.m.f. group $\operatorname{Aut}\left(B_{16}\right)<\mathrm{GL}_{16}(\mathbb{Q})$ with index $2^{10} \cdot 16$ !.

Hence one has to proceed as in the classification of the r.i.m.f. matrix groups. The r.i.m.f. or s.i.m.f. matrix groups are full automorphism groups of some lattices. Further, two such groups are conjugate if and only if certain lattices are isometric.

The concept of primitivity is the key ingredient to these classifications since it has some important consequences. A symplectic matrix group is called symplectic primitive if it is not contained (up to conjugacy) in a wreath product of some symplectic matrix group. The restriction of the natural character of a symplectic primitive irreducible maximal finite (s.p.i.m.f.) matrix group $G<\mathrm{GL}_{2 n}(\mathbb{Q})$ to a normal subgroup $N$ is a multiple of a single rationally irreducible character of $N$. Furthermore, if $G$ is s.p.i.m.f. then there exists a finite list of candidates (depending only on $n$ ) for the generalized Fitting subgroup $F^{*}(G)$ of $G$. The possible Fitting subgroups are given by a theorem of Hall. The possible layers (central products of quasisimple groups) can be taken from Hiss and Malle [HM01] which is based of the atlas of finite simple groups $\mathrm{CCN}^{+} 85$ ]. So the completeness of this list depends on the classification of the finite simple groups.

Then it remains to construct all possible extensions $G$ of $F^{*}(G)$ up to conjugacy. There are several shortcuts to find $G$ or at least a large (normal) subgroup of $G$. A very useful tool is the so-called generalized Bravais group. If $N$ is a normal subgroup of a s.p.i.m.f. matrix group $G<\mathrm{GL}_{2 n}(\mathbb{Q})$, then the generalized Bravais group $\mathcal{B}^{\circ}(N)$ contains $N$ and can be computed directly from $N$. Further, $N$ and $\mathcal{B}^{\circ}(N)$ have the same commuting algebras and $\mathcal{B}^{\circ}(N)$ is a normal subgroup of $G$.

It turns out that, like in the classification of r.i.m.f. matrix groups, the number of conjugacy classes of s.i.m.f. subgroups of $\mathrm{GL}_{2 n}(\mathbb{Q})$ varies greatly depending on whether $n$ is divisible by a large power of 2 or not. This is due to the fact that the list of possible Fitting subgroups is much larger in the first case. For example, there are 91 conjugacy classes of s.i.m.f. groups in $\mathrm{GL}_{16}(\mathbb{Q})$, but there are only 5 conjugacy classes in dimension 14 .

This classification relies on calculations (computations of automorphism groups and invariant forms, ideal arithmetic, ...) that require the use of a computer algebra system. All these calculations were performed in MAGMA [BCP97] since this system is extensible and it provides almost all necessary algorithms for lattices, group theory, number fields and (quaternion) algebras. In particular, MAGMA contains an implementation of the algorithm of Plesken and Souvignier [PS97] for computing automorphism groups and isometries of lattices.

This thesis is organized as follows. Section 2.1 starts with basic definitions and gives a first overview of symplectic matrix groups. It also recalls the definition of generalized Bravais groups and elaborates the general outline of the classification. Section 2.2 
explains the so-called " $m$-parameter argument" which allows us to construct all s.i.m.f. supergroups of a given irreducible matrix group $U$ whose commuting algebra is a field. We also give an algorithm which computes all s.i.m.f. supergroups $G$ of $U$ if the commuting algebra of $U$ is a quaternion algebra provided that $U \unlhd G$ and $[G: U]$ is a power of 2 . This section also contains some methods that can be used to rule out several candidates for normal subgroups. Section 2.3 explains the sublattice algorithm. Section 2.4 describes several constructions and notational conventions for maximal finite matrix groups. Finally, Section 2.5 contains a complete list of all possible generalized Fitting subgroups of s.p.i.m.f. matrix groups up to dimension $2 n=22$.

Chapter 3 describes some infinite families of s.i.m.f. matrix groups. In particular, all s.i.m.f. subgroups of $\mathrm{GL}_{p-1}(\mathbb{Q})$ and $\mathrm{GL}_{p+1}(\mathbb{Q})$ whose orders are divisible by a prime $p \geq 5$ are determined.

Chapter 4 deals with the classification of the s.i.m.f. subgroups of $\mathrm{GL}_{2 n}(\mathbb{Q})$ for $1 \leq n \leq 11$. For each dimension, the classification of the conjugacy classes of s.i.m.f. subgroups is given as a table. For each class, it contains a name that describes how a representative of that class can be constructed (from $p$-subgroups, quasisimple groups or smaller maximal finite matrix groups by taking generalized Bravais groups, tensor products, wreath products or group extensions). The table also contains the following invariants of each conjugacy class: group order, commuting algebra, number of isomorphism classes of invariant lattices and further information on certain invariant lattices. Together these invariants provide an easy method for recognizing the conjugacy class of a given s.i.m.f. matrix group.

For each conjugacy class, the appendix contains a symmetric positive definite and a skewsymmetric form such that the automorphism group of the standard lattice with respect to these two forms represents that particular class. These forms are also available in a MAGMA [BCP97] readable format from

$$
\text { http://www .math.rwth-aachen.de/ Markus.Kirschmer/symplectic/. }
$$

First of all, I would like to express my deepest gratitude to Prof. Dr. Gabriele Nebe. This thesis would have been impossible without her guidance and advice.

I want to thank the Deutsche Forschungsgesellschaft for my scholarship in the research training group "Hierarchie und Symmetrie in mathematischen Modellen".

Furthermore, I would like to thank my colleagues especially Dr. Matthias Künzer, Annika Günther, Elisabeth Nossek, Georg Deifuss, Kristina Schindelar and Moritz Schröer for numerous discussions and social events. 


\section{Chapter 2}

\section{Methods}

\subsection{Definitions}

\subsubsection{Symplectic matrix groups}

This thesis classifies the conjugacy classes of all maximal finite symplectic subgroups of $\mathrm{GL}_{m}(\mathbb{Q})$ for $1 \leq m \leq 22$. Two very important tools for the classification are the form spaces and the commuting algebras:

Definition 2.1.1 Let $G \leq \mathrm{GL}_{m}(\mathbb{Q})$.

(a) The $\mathbb{Q}$-space of $G$-invariant forms is given by

$$
\mathcal{F}(G):=\left\{F \in \mathbb{Q}^{m \times m} \mid g F g^{\text {tr }}=F \text { for all } g \in G\right\} .
$$

Further $\mathcal{F}_{\text {sym }}(G), \mathcal{F}_{>0}(G)$ and $\mathcal{F}_{\text {skew }}(G)$ denote the subset of symmetric, symmetric positive definite and skewsymmetric $G$-invariant forms respectively.

The group $G$ is called symplectic if $\mathcal{F}_{\text {skew }}(G)$ contains an invertible element and $G$ is said to be uniform if $\operatorname{dim}_{\mathbb{Q}}\left(\mathcal{F}_{\text {sym }}(G)\right)=1$.

(b) The enveloping algebra $\bar{G}$ of $G$ is the subspace of $\mathbb{Q}^{m \times m}$ generated by the matrices in $G$. Further

$$
\operatorname{End}(\bar{G}):=C_{\mathbb{Q}^{m \times m}}(G):=\left\{X \in \mathbb{Q}^{m \times m} \mid X g=g X \text { for all } g \in G\right\}
$$

is the endomorphism ring or commuting algebra of $G$.

Remark 2.1.2 Let $G<\mathrm{GL}_{m}(\mathbb{Q})$.

(a) If $F \in \mathcal{F}(G)$ is invertible, then $\operatorname{End}(\bar{G}) \rightarrow \mathcal{F}(G), e \mapsto e F$ is an isomorphism of $\mathbb{Q}$-spaces. Its inverse is given by $\mathcal{F}(G) \rightarrow \operatorname{End}(\bar{G}), F^{\prime} \mapsto F^{\prime} F^{-1}$.

(b) If $G$ is finite, then $\sum_{g \in G} g g^{\text {tr }} \in \mathcal{F}_{>0}(G)$. In particular, $\mathcal{F}(G) \simeq \operatorname{End}(\bar{G})$. 
Remark 2.1.3 Let $J_{n}:=\left(\begin{array}{cc}0 & I_{n} \\ -I_{n} & 0\end{array}\right) \in \mathrm{GL}_{2 n}(\mathbb{Q})$.

(a) If $G<\mathrm{GL}_{m}(\mathbb{Q})$ is symplectic, them $m$ is even.

(b) $\operatorname{Sp}_{2 n}(\mathbb{Q}):=\left\{g \in \mathrm{GL}_{2 n}(\mathbb{Q}) \mid g J_{n} g^{\text {tr }}=J_{n}\right\}$ is a subgroup of $\mathrm{SL}_{2 n}(\mathbb{Q})$.

(c) An invertible matrix $S \in \mathrm{GL}_{2 n}(\mathbb{Q})$ is skewsymmetric if and only if $S=J_{n}^{x}$ for some $x \in \mathrm{GL}_{2 n}(\mathbb{Q})$. In particular, a finite subgroup $G<\mathrm{GL}_{2 n}(\mathbb{Q})$ is symplectic if and only if there exists some $x \in \mathrm{GL}_{2 n}(\mathbb{Q})$ such that $G^{x}<\operatorname{Sp}_{2 n}(\mathbb{Q})$.

Proof: See for example [Art57, Theorems 3.7 and 3.25].

So any conjugacy class of (maximal) finite symplectic subgroups of $\mathrm{GL}_{2 n}(\mathbb{Q})$ has a representative in $\operatorname{Sp}_{2 n}(\mathbb{Q})$.

The most important computational tool for the enumeration of the maximal finite subgroups of $\mathrm{GL}_{m}(\mathbb{Q})$ are the $G$-invariant lattices and automorphism groups. They are defined as follows.

Definition 2.1.4 Let $R$ be a Dedekind ring such that its quotient field $K$ is a number field.

(a) An $R$-lattice is a finitely generated $R$-module in some vector space over $K$.

(b) An $R$-order is a subring of a finite dimensional $K$-algebra that is also an $R$-lattice.

(c) If $\Lambda$ is a $\mathbb{Z}$-order in $\mathbb{Q}^{m \times m}$ then

$$
\mathcal{Z}(\Lambda):=\left\{L \subset \mathbb{Q}^{1 \times m} \mid L \text { is } \mathbb{Z} \text {-lattice of rank } m \text { with } L x \subseteq L \text { for all } x \in \Lambda\right\}
$$

denotes the set of all $\Lambda$-invariant lattices.

Similarly if $G<\mathrm{GL}_{m}(\mathbb{Q})$, then

$$
\mathcal{Z}(G):=\left\{L \subset \mathbb{Q}^{1 \times m} \mid L \text { is a } \mathbb{Z} \text {-lattice of rank } m \text { with } L x \subseteq L \text { for all } x \in G\right\}
$$

is the set of all $G$-invariant lattices.

(d) For a $\mathbb{Z}$-lattice $L \subset \mathbb{Q}^{1 \times m}$ of rank $m$, a set $\mathcal{F} \subseteq \mathbb{Q}^{m \times m}$ and some subfield $K$ of $\mathbb{Q}^{m \times m}$ let

$\operatorname{Aut}_{K}(L, \mathcal{F})=\left\{g \in \mathrm{GL}_{m}(\mathbb{Q}) \mid L g=L, g F g^{\text {tr }}=F, g c=c g\right.$ for all $\left.F \in \mathcal{F}, c \in K\right\}$

be the group of $K$-linear automorphisms of $L$ with respect to $\mathcal{F}$. If $\mathcal{F}=\{F\}$ consists only of one form, we write $\operatorname{Aut}_{K}(L, F)$ instead of $\operatorname{Aut}_{K}(L,\{F\})$ and if $K \simeq \mathbb{Q}$, we will omit the subscript $K$.

Note that, if $\mathcal{F}$ contains a positive definite symmetric matrix, then

$$
\operatorname{Aut}_{K}(L, \mathcal{F})=\operatorname{Aut}(L,\{x F \mid x \in K, F \in \mathcal{F}\})
$$

and we will switch frequently between these two notations in the sequel. 
We are now ready to give a characterization of (maximal) finite rational matrix groups.

\section{Remark 2.1.5}

(a) Let $L \subseteq \mathbb{Q}^{1 \times m}$ be a $\mathbb{Z}$-lattice of rank $m$ and $F \in \mathbb{Q}^{m \times m}$ be symmetric and positive definite. Then $\operatorname{Aut}(L, F)$ is finite.

(b) A group $G<\mathrm{GL}_{m}(\mathbb{Q})$ is finite if and only if $\mathcal{F}_{>0}(G)$ and $\mathcal{Z}(G)$ are nonempty.

(c) If $G<\mathrm{GL}_{m}(\mathbb{Q})$ is finite then $S:=\left\{\operatorname{Aut}(L, F) \mid(L, F) \in \mathcal{Z}(G) \times \mathcal{F}_{>0}(G)\right\}$ contains all maximal finite supergroups of $G$.

In particular, $G$ is maximal finite if and only if $S=\{G\}$. The maximal finite subgroups of $\mathrm{GL}_{m}(\mathbb{Q})$ have been classified in [BBNZ77, Ple91, NP95, Neb95, Neb96] for all $m<32$.

Proof: (a) The norm induced by $F$ on $\mathbb{R}^{1 \times m}$ is equivalent to the maximum norm. So there exist only finitely many vectors in $L$ of a given length. Hence there exist only finitely many possible images for some fixed basis vectors of $L$ under an automorphism. (b) If $G$ is finite then $\sum_{g \in G} g g^{\operatorname{tr}} \in \mathcal{F}_{>0}(G)$ and $\sum_{g \in G} L g \in \mathcal{Z}(G)$ for any $\mathbb{Z}$-lattice $L$ of rank $m$. Conversely, if $(L, F) \in \mathcal{Z}(G) \times \mathcal{F}_{>0}(G)$ then $G \leq \operatorname{Aut}(L, F)$ is finite.

In the same spirit, we want to characterize the maximal finite symplectic subgroups of $\mathrm{GL}_{2 n}(\mathbb{Q})$. First we will give this characterization for rationally irreducible matrix groups, where irreducibility is defined as follows:

Definition 2.1.6 A matrix group $G<\mathrm{GL}_{m}(K)$ is called $K$-irreducible (or just irreducible) if the natural representation of $G$ is irreducible over $K$. In the case $K=\mathbb{Q}$ we also use the phrase "rationally irreducible".

As Remark 2.1.2 shows, there is a tight connection between the form space $\mathcal{F}(G)$ and the commuting algebra $\operatorname{End}(\bar{G})$. In particular, symplectic matrix groups can also be characterized by their endomorphism rings as Lemma 2.1.9 shows.

But before we state this lemma, we recall two well known facts.

Definition and Remark 2.1.7 Let $G<\mathrm{GL}_{m}(\mathbb{Q})$ be irreducible and finite. Then $E:=\operatorname{End}(\bar{G})$ is a skewfield of dimension $e:=\operatorname{dim}_{\mathbb{Q}}(E)$ say. Suppose that $S \subseteq E$ is a simple subalgebra with $s:=\operatorname{dim}_{\mathbb{Q}}(S)$. By the double centralizer property, we have a sequence of $\mathbb{Q}$-algebra monomorphisms

$$
\bar{G}=C_{\mathbb{Q}^{m \times m}}(E) \simeq\left(E^{o}\right)^{\frac{m}{e} \times \frac{m}{e}} \hookrightarrow\left(S^{o}\right)^{\frac{m}{s} \times \frac{m}{s}} \stackrel{\triangle^{\mathbb{Q}}}{\hookrightarrow} \mathbb{Q}^{m \times m}
$$

where the superscript ${ }^{o}$ denotes the opposite algebra. Let $\triangle_{S}$ be the composition of the first two morphisms. Then the character of $\triangle_{S}(G)<\mathrm{GL} \frac{m}{s}\left(S^{o}\right)$ is not uniquely determined by $G$, but the composition $\triangle^{\mathbb{Q}} \circ \triangle_{S}$ is conjugation by some $x \in \mathrm{GL}_{m}(\mathbb{Q})$ according to the Skolem-Noether theorem. In particular, $G$ and $\triangle^{\mathbb{Q}}\left(\triangle_{S}(G)\right)$ are conjugate and $\triangle_{S}(G)$ is irreducible. 
Remark 2.1.8 Let $K$ be a number field of degree $d=\operatorname{dim}_{\mathbb{Q}}(K)$. Further let $H_{1}, H_{2}<$ $\mathrm{GL}_{m}(K)$ be irreducible and finite. If $H_{1}$ and $H_{2}$ are conjugate in $\mathrm{GL}_{m}(K)$ then $\triangle^{\mathbb{Q}}\left(H_{1}\right)$ and $\triangle^{\mathbb{Q}}\left(H_{2}\right)$ are also conjugate in $\mathrm{GL}_{m d}(\mathbb{Q})$. Conversely, if $\triangle^{\mathbb{Q}}\left(H_{1}\right)$ and $\triangle^{\mathbb{Q}}\left(H_{2}\right)$ are conjugate then the natural characters of $H_{1}$ and $H_{2}$ must be algebraically conjugate.

Lemma 2.1.9 Let $G<\mathrm{GL}_{m}(\mathbb{Q})$ be irreducible and finite. Further let $E:=\operatorname{End}(\bar{G})$ and denote by $K$ the center of $E$.

(a) Let $F \in \mathcal{F}_{>0}(G)$ and $e \in E$. If eF $\in \mathcal{F}(G)$ is symmetric (skewsymmetric) then the subfield $\mathbb{Q}(e) \leq E$ is totally real (totally complex).

Conversely, if $\mathbb{Q}(e) \leq K$ is totally real, then eF is symmetric.

(b) The following statements are equivalent:

(1) $G$ is symplectic.

(2) E contains a (minimal) totally complex subfield.

(3) There exists a (minimal) totally complex number field $K^{\prime}$ of degree $d \mid m$ and some $H<\mathrm{GL}_{\frac{m}{d}}\left(K^{\prime}\right)$ such that $G$ is conjugate to $\triangle^{\mathbb{Q}}(H)$ in $\mathrm{GL}_{m}(\mathbb{Q})$.

In particular, $G$ is a symplectic irreducible maximal finite (s.i.m.f.) subgroup of $\mathrm{GL}_{m}(\mathbb{Q})$ if and only if $G=\operatorname{Aut}_{K^{\prime}}(L, F)$ for all $(L, F) \in \mathcal{Z}(G) \times \mathcal{F}_{>0}(G)$ and for all minimal totally complex subfields $K^{\prime}$ of $\operatorname{End}(\bar{G})$.

(c) Each $\tilde{F} \in \mathcal{F}_{>0}(G)$ induces involutions on $E, \bar{G}$ and $K$ via $x \mapsto x^{\circ}:=\tilde{F} x^{\operatorname{tr}} \tilde{F}^{-1}$.

The involutions on $\bar{G}$ and $K$ do not dependent on the form $\tilde{F}$ and the fixed field of ${ }^{\circ}: K \rightarrow K$ is the maximal totally real subfield $K^{+}$of $K$.

Further, $K$ is either totally real or a $C M$-field (i.e. $K$ is totally complex and $\left.\left[K: K^{+}\right]=2\right)$. In particular, ${ }^{\circ}$ is the (unique) complex conjugation on $K$.

Proof:

(a) If $e F$ is symmetric, then $e F=F e^{\text {tr }}$ shows that $e$ is a selfadjoint automorphism of the Euclidean space $\left(\mathbb{R}^{1 \times m}, F\right)$. So it generates a totally real field. A similar argument holds for skewsymmetric forms.

Suppose now $e \in K$ is totally real. Since $\mathcal{F}(G)$ is closed under taking transposes, it decomposes into $\mathcal{F}_{\text {sym }}(G) \oplus \mathcal{F}_{\text {skew }}(G)$. Hence $e F=e_{1} F+e_{2} F$ with $e_{1} F$ symmetric and $e_{2} F$ skewsymmetric. In particular $e_{1}$ is totally real and $e_{2}$ totally complex by the above. But $e_{2}=e-e_{1} \in \mathbb{Q}\left(e, e_{1}\right)$ is contained in a totally real field. So $e_{2}=0$.

(b) Part (a) shows (1) $\Rightarrow(2)$. For the converse fix $F \in \mathcal{F}_{>0}(G)$ and note that $E \otimes_{\mathbb{Q}} \mathbb{R}$ cannot be a sum of copies of $\mathbb{R}$. Thus $G$ fixes at least one real valued skewsymmetric form. Hence $\mathcal{F}_{\text {skew }}(G) \subset\{e F \mid e \in E\}$ contains a nonzero element, $S$ say. Since $E$ is a skewfield, the form $S$ is already invertible. So $G$ is symplectic. For $(2) \Rightarrow(3)$ one can choose $H:=\triangle_{K^{\prime}}(G)$ where $K^{\prime}$ is a minimal totally complex subfield of $E$. For the converse, note that $\operatorname{End}\left(\overline{\triangle^{\mathbb{Q}}(H)}\right) \simeq E$ has a subfield isomorphic to $K^{\prime}$. 
(c) It is clear that ${ }^{\circ}$ is an involution on $\bar{G}$ and $E$. Thus it is an automorphism on $E \cap \bar{G}=K$. Since any $\tilde{F} \in \mathcal{F}_{>0}(G)$ is of the form $e F$ for some $e \in E$, it follows that $^{\circ}: \bar{G} \rightarrow \bar{G}$ does not depend on $\tilde{F}$. By part (a) it also follows that $K^{+}$is the fixed field of ${ }^{\circ}: K \rightarrow K$.

The field $K$ is the character field of some complex constituent of the natural representation of $G$. So $K / \mathbb{Q}$ is Galois. In particular, if $K$ has an embedding into $\mathbb{R}$ then all embeddings $K \rightarrow \mathbb{C}$ would be real. So $K$ is either totally real or totally complex and the index $\left[K: K^{+}\right]$equals the order of ${ }^{\circ}: K \rightarrow K$.

From this result, we immediately obtain the following corollary. It shows that we only have to classify the conjugacy classes of s.i.m.f. matrix groups to get the classification of the conjugacy classes for all maximal finite symplectic matrix groups.

Corollary 2.1.10 If $G<\mathrm{GL}_{m}(\mathbb{Q})$ is maximal finite symplectic, then the natural representation $\triangle: G \rightarrow \mathrm{GL}_{m}(\mathbb{Q})$ splits into a sum of pairwise nonisomorphic irreducible representations $\triangle_{i}: G \rightarrow \mathrm{GL}_{m_{i}}(\mathbb{Q})$ and each group $\triangle_{i}(G)$ is s.i.m.f..

Proof: We have a decomposition $\triangle=\sum_{i=1}^{s} n_{i} \triangle_{i}$ into irreducible and pairwise nonisomorphic representations $\triangle_{i}: G \rightarrow \mathrm{GL}_{m_{i}}(\mathbb{Q})$. Hence we may assume that $G<$ $\left\{\operatorname{Diag}\left(x_{1}, \ldots, x_{m}\right) \mid x_{i} \in \mathrm{GL}_{n_{i} m_{i}}(\mathbb{Q})\right\}$. Hence $\operatorname{End}(\bar{G})$ and thus $\mathcal{F}(G)$ are given by block diagonal matrices and each group $n_{i} \triangle_{i}(G)$ is maximal finite symplectic since $G$ fixes an invertible skewsymmetric form $S$.

Suppose now $n_{i}>1$ for some $i$. If $n_{i}>2$ then $\left(n_{i}-2\right) \triangle_{i}(G)$ is symplectic. This is clearly true if $\triangle_{i}(G)$ fixes a skewsymmetric form. In the other case, $E_{i}:=\operatorname{End}(\overline{\triangle(G)})$ is a totally real field and $S$ is the tensor product of an invertible skewsymmetric matrix in $E_{i}^{n_{i} \times n_{i}}$ with some $F \in \mathcal{F}_{>0}\left(\triangle_{i}(G)\right)$. Thus $n_{i}$ is even and $\left(n_{i}-2\right) \triangle_{i}(G)$ is symplectic. So we may suppose that $n_{i}=2$. But then $2 \triangle_{i}(G)$ is properly contained in $H:=$ $\left\langle 2 \triangle_{i}(G),\left(\begin{array}{ll}0 & \zeta \\ 1 & 0\end{array}\right)\right\rangle$ where $\zeta \in E_{i}$ is a torsion unit of maximal order. One checks that $H$ is irreducible and thus symplectic by the previous lemma since its endomorphism ring contains a cyclotomic subfield.

Remark 2.1.11 Suppose $K$ is a minimal totally complex number field. Lemma 2.1.9 and Remark 2.1 .8 show that the classification of all s.i.m.f. $\operatorname{subgroups}$ of $\mathrm{GL}_{m \operatorname{dim}_{\mathbb{Q}}(K)}(\mathbb{Q})$ yields all conjugacy classes of maximal finite $K$-irreducible subgroups $H<\mathrm{GL}_{m}(K)$ satisfying $\operatorname{End}\left(\overline{\triangle^{\mathbb{Q}}(H)}\right) \simeq K$. If $K \not \neq \operatorname{End}\left(\overline{\triangle^{\mathbb{Q}}(H)}\right)$, then two problems may arise:

- If $H<\mathrm{GL}_{m}(K)$ is $K$-irreducible and maximal finite then $\triangle^{\mathbb{Q}}(H)$ might be reducible over $\mathbb{Q}$ as the example $H=\langle \pm 1\rangle<\mathrm{GL}_{1}(\mathbb{Q}(\sqrt{-d}))$ for every squarefree $d \in \mathbb{Z}_{>0} \backslash\{1,3\}$ shows.

- Even if $H<\mathrm{GL}_{m}(K)$ is $K$-irreducible and maximal finite such that $\triangle^{\mathbb{Q}}(H)$ is rationally irreducible, it might not be maximal finite symplectic, as the following example shows:

Let $\mathcal{Q}_{\infty, 2}$ be the quaternion algebra over $\mathbb{Q}$ that is only ramified at 2 and the infinite place. Denote by $\mathfrak{M}$ a maximal order (it is unique up to conjugacy). Then the torsion subgroup $\mathfrak{M}^{*, 1}$ is isomorphic to $\mathrm{SL}_{2}(3)$. We denote 
by $\infty, 2\left[\mathrm{SL}_{2}(3)\right]_{1}:=\triangle^{\mathbb{Q}}\left(\mathfrak{M}^{*, 1}\right)$ the corresponding subgroup of $\operatorname{Sp}_{4}(\mathbb{Q})$. By Theorem 4.3 .1 this group has (up to conjugacy) three s.i.m.f. supergroups namely ${ }_{i}\left[\left(D_{8} \otimes C_{4}\right) \cdot S_{3}\right]_{2}, \sqrt{-2}\left[\mathrm{GL}_{2}(3)\right]_{2}$ and ${ }_{\infty, 2}\left[\mathrm{SL}_{2}(3)\right]_{1} \circ C_{3}$. These groups have $\mathbb{Q}(i)$, $\mathbb{Q}(\sqrt{-2})$ and $\mathbb{Q}(\sqrt{-3})$ as commuting algebras respectively. Let $K=\mathbb{Q}(\sqrt{-d})$ be any splitting field of $\mathcal{Q}_{\infty, 2}$ such that $d \notin\{1,2,3\}$ (for example $K=\mathbb{Q}(\sqrt{-5})$ ). Then $H:=\triangle^{K}\left(\mathfrak{M}^{*, 1}\right)$ is a $K$-irreducible maximal finite subgroup of $\mathrm{GL}_{2}(K)$ but ${ }_{\infty, 2}\left[\mathrm{SL}_{2}(3)\right]_{1}=\triangle^{\mathbb{Q}}(H)$ has $\mathcal{Q}_{\infty, 2}$ as commuting algebra and this group is not s.i.m.f..

By Remark 2.1.3 any conjugacy class of maximal finite symplectic matrix groups contains a representative in $\operatorname{Sp}_{2 n}(\mathbb{Q})$ for some $n \in \mathbb{Z}$. So one might ask to find all (maximal) finite subgroups of $\operatorname{Sp}_{2 n}(\mathbb{Q})$ up to conjugacy in $\operatorname{Sp}_{2 n}(\mathbb{Q})$. The following remark shows that there are infinitely many of these classes:

Lemma 2.1.12 Let $G<\operatorname{Sp}_{2 n}(\mathbb{Q})$ be finite such that $E:=\operatorname{End}(\bar{G})$ is a field. Denote by $E^{+}$its maximal totally real subfield.

(a) Let $t_{1}, \ldots, t_{s}$ be representatives of $N_{\mathrm{GL}_{2 n}(\mathbb{Q})}(G) /\left\langle G, E^{*}\right\rangle \leq \operatorname{Out}(G)$. For $1 \leq i \leq$ $s$ let $e_{i}:=t_{i} J_{n} t_{i}{ }^{\operatorname{tr}} J_{n}^{-1} \in\left(E^{+}\right)^{*}$. Then $\mathcal{S}:=\bigcup_{i=1}^{s} e_{i} \mathrm{Nr}_{E / E^{+}}\left(E^{*}\right)$ is independent of the choice of the $t_{i}$.

(b) Let $H:=\left\{x \in \mathrm{GL}_{2 n}(\mathbb{Q}) \mid G^{x} \leq \mathrm{Sp}_{2 n}(\mathbb{Q})\right\}$. Then $\varphi: H \rightarrow\left(E^{+}\right)^{*}, x \mapsto x J_{n} x^{\text {tr }} J_{n}^{-1}$ is surjective.

(c) Let $x \in H$. Then $G$ and $G^{x}$ are conjugate in $\operatorname{Sp}_{2 n}(\mathbb{Q})$ if and only if $\varphi(x) \in \mathcal{S}$.

(d) Suppose $E$ is an imaginary quadratic number field. Then $H$ and $\mathcal{S}$ are groups and $\varphi$ is a homomorphism of groups. Let $x, y \in H$. Then $G^{x}$ and $G^{y}$ are conjugate in $\operatorname{Sp}_{2 n}(\mathbb{Q})$ if and only if $\varphi(x) \mathcal{S}=\varphi(y) \mathcal{S}$.

Moreover, the $\mathrm{GL}_{2 n}(\mathbb{Q})$ conjugacy class of $G$ intersected with $\mathrm{Sp}_{2 n}(\mathbb{Q})$ (i.e. the set $\left\{G^{x} \mid x \in H\right\}$ ) decomposes into infinitely many $\operatorname{Sp}_{2 n}(\mathbb{Q})$ conjugacy classes and there is a bijection between these classes and $\mathbb{Q}^{*} / \mathcal{S}$.

Proof:

(a) Each $t_{i}$ normalizes $G$, thus it acts on $\mathcal{F}_{\text {skew }}(G)=\left\{e J_{n} \mid e \in E^{+}\right\}$. Hence $e_{i} \in E^{+}$. Moreover if $t_{i}^{\prime}$ and $t_{i}$ represent the same coset, then $t_{i}^{\prime}=$ get $_{i}$ for some $g \in G$ and $e \in E$. In particular $t_{i}^{\prime} J_{n} t_{i}^{\text {tr }}=g e e_{i} J_{n} e^{\mathrm{tr}} g^{\mathrm{tr}}=e_{i} \mathrm{Nr}_{E / E^{+}}(e) J_{n}$.

(b) Let $x \in \mathrm{GL}_{2 n}(\mathbb{Q})$. Then $x \in H$ if and only if $J_{n} \in \mathcal{F}_{\text {skew }}\left(G^{x}\right)=\left\{x^{-1} e J_{n} x^{-\operatorname{tr}}\right.$ $\left.e \in E^{+}\right\}$. Thus $x \in H$ implies $\varphi(x) \in\left(E^{+}\right)^{*}$. So $\varphi$ is well defined. Finally, it follows from Remark 2.1.3(c) and the above that $\varphi$ is surjective.

(c) Suppose $G^{x}=G^{y}$ for some $y \in \operatorname{Sp}_{2 n}(\mathbb{Q})$. Then $x y^{-1}$ normalizes $G$. So $x y^{-1}=e g t_{i}$ for some $1 \leq i \leq s, e \in E$ and $g \in G$. Then $\varphi(x) J_{n}=x J_{n} x^{\text {tr }}=$ $e g t_{i} y J_{n} y^{\mathrm{tr}} t_{i}{ }^{\mathrm{tr}} g^{\mathrm{tr}} e^{\mathrm{tr}}=\mathrm{Nr}_{E / E^{+}}(e) e_{i} J_{n}$ shows that $\varphi(x) \in \mathcal{S}$.

Conversely, if $\varphi(x) \in \mathcal{S}$ then $\varphi(x)=\mathrm{Nr}_{E / E^{+}}(e) e_{i}$ for some $1 \leq i \leq s$ and $e \in E$. Hence $x J_{n} x^{\text {tr }}=e t_{i} J_{n} t_{i}^{\text {tr }} e^{\text {tr }}$ implies that $y:=t_{i}^{-1} e^{-1} x \in \operatorname{Sp}_{2 n}(\mathbb{Q})$. Further $G^{y}=\left(G^{t_{i}^{-1} e^{-1}}\right)^{x}=G^{x}$. 
(d) Since $E^{+}=\mathbb{Q}$ consists only of scalar matrices, one checks then $H$ and $\mathcal{S}$ are groups and $\varphi$ is a morphism. Further $G^{x}=\left(G^{y}\right)^{z}$ for some $z \in \operatorname{Sp}_{2 n}(\mathbb{Q})$ if and only if $\varphi\left(x z^{-1} y^{-1}\right) \in \mathcal{S}$. Since $\varphi(z)=1$ this is equivalent to $\varphi(x) \mathcal{S}=\varphi(y) \mathcal{S}$. It remains to show that $\mathbb{Q}^{*} / \mathcal{S}$ is infinite. This follows from $\left[\mathcal{S}: \operatorname{Nr}_{E / \mathbb{Q}}\left(E^{*}\right)\right] \leq$ $|\operatorname{Out}(G)|$ and the fact that $\mathbb{Q}^{*} / \mathrm{Nr}_{E / E^{+}}\left(E^{*}\right)$ is always an infinite group. A proof of this statement is given in [Ste89, pg. 208] and I would like to thank Hans Opolka for pointing out this reference.

From now on, conjugacy means conjugacy in $\mathrm{GL}_{2 n}(\mathbb{Q})$. Further, since we want to classify the conjugacy classes of maximal finite symplectic matrix groups, we may w.l.o.g. suppose that a given symplectic matrix group is contained in $\operatorname{Sp}_{2 n}(\mathbb{Q})$. I.e. we write $G<\operatorname{Sp}_{2 n}(\mathbb{Q})$ to indicate that $G<\mathrm{GL}_{2 n}(\mathbb{Q})$ is symplectic.

\subsubsection{Primitivity}

To classify all maximal finite symplectic matrix groups, it suffices to classify only s.i.m.f. matrix groups as Corollary 2.1.10 shows. In this section we will reduce the number of groups to consider even further.

Definition 2.1.13 Let $K$ be a number field. A $K$-irreducible subgroup $G<\mathrm{GL}_{m}(K)$ is called primitive, if $G$ is not conjugate to a subgroup of the wreath product

$$
\left.H 々 S_{k}:=\left\langle\operatorname{Diag}\left(h_{1}, \ldots, h_{k}\right), P \otimes I_{\frac{m}{k}}\right| h_{i} \in H, P \text { a } k \times k \text { permutation matrix }\right\rangle
$$

for some $H<\operatorname{GL}_{\frac{m}{k}}(K)$ where $k$ is a divisor of $m$.

Similarly, a $\mathbb{Q}$-irreducible symplectic subgroup $G<\mathrm{GL}_{2 n}(\mathbb{Q})$ is called symplectic primitive, if $G$ is not conjugate to a subgroup of $H<S_{k}$ for some $H<\operatorname{Sp}_{\frac{2 n}{k}}(\mathbb{Q})$ where $k \mid n$.

Remark 2.1.14 A rationally irreducible symplectic subgroup $G<\mathrm{GL}_{2 n}(\mathbb{Q})$ is symplectic primitive if and only if $\triangle_{K}(G)$ is primitive for all minimal totally complex subfields $K$ of $\operatorname{End}(\bar{G})$.

The concept of primitivity is a key ingredient in the determination of all irreducible finite matrix groups. It has some important consequences for normal subgroups.

Theorem 2.1.15 ([NP95, Lemma (III.1)]) Let $G<\mathrm{GL}_{m}(K)$ be a rationally irreducible primitive matrix group and $N \unlhd G$. Then $\langle N\rangle_{K} \leq K^{m \times m}$ is a simple algebra or equivalently, the natural $K N$-module $K^{1 \times m}$ splits into a direct sum of $k$ isomorphic $K N$-modules of dimension $\frac{m}{k}$. 
Proof: The group $G$ acts on $N$ by conjugation. Hence it also acts on the set of central primitive idempotents of $\langle N\rangle_{K}$. Thus $G$ permutes the homogeneous components of the natural $K N$-module $K^{1 \times m}$. But since $G$ is primitive, there can only be one such component.

Corollary 2.1.16 ([NP95, (III.1)-(III.3)]) Let $G<\mathrm{GL}_{2 n}(\mathbb{Q})$ be rationally irreducible and symplectic primitive. Further let $p$ be a prime divisor of $|G|$.

(a) If $N \unlhd G$ then $\bar{N} \leq \mathbb{Q}^{2 n \times 2 n}$ is a simple subalgebra.

(b) If $O_{p}(G) \neq 1$ then there exists some $k \geq 0$ such that $p^{k}(p-1)$ divides $2 n$.

(c) All abelian characteristic subgroups of $O_{p}(G)$ are cyclic.

Proof:

(a) Let $K<\operatorname{End}(\bar{G})$ be any minimal totally complex subfield. Let $\left\{f_{1}, \ldots, f_{r}\right\}$ and $\left\{e_{1}, \ldots, e_{s}\right\}$ be the central primitive idempotents of the enveloping algebras $\bar{N}$ and $\langle G\rangle_{K}$ respectively. Let $\chi_{i}$ denote the character corresponding to a simple $\langle G\rangle_{K} e_{i}$ module and let $L=\mathbb{Q}\left(\chi_{1}, \ldots, \chi_{s}\right) \subseteq K$ be their character field. Then $L / \mathbb{Q}$ is Galois and $e_{i}=\frac{\chi_{i}(1)}{|G|} \sum_{g \in G} \chi_{i}\left(g^{-1}\right) g \in\langle G\rangle_{L}$. Since $G$ is irreducible, $\left\{e_{1}, \ldots, e_{s}\right\}$ is a Galois orbit under $\operatorname{Gal}(L / \mathbb{Q})$. For any $1 \leq j \leq r$ there exists some $i$ such that $e_{i} f_{j} \neq 0$. Since $f_{j} \in \bar{N}$ is fixed under $\operatorname{Gal}(L / \mathbb{Q})$, we get that $e_{i} f_{j} \neq 0$ for all $i, j$. The enveloping algebra $\left\langle\triangle_{K}(G)\right\rangle_{K}$ is isomorphic to $\langle G\rangle_{K} e_{i}$ for some $i$. Now $\left\{e_{i} f_{1}, \ldots, e_{i} f_{r}\right\}$ is a set of central idempotents of $\langle N\rangle_{K} e_{i} \simeq\left\langle\triangle_{K}(N)\right\rangle_{K}$. But $\triangle_{K}(G)$ is primitive and therefore $\left\langle\triangle_{K}(N)\right\rangle_{K}$ is a simple algebra by Theorem 2.1.15. This shows $r=1$, since no $e_{i} f_{j}$ vanishes.

(b) By (a), $O_{p}(G)$ has a rationally irreducible representation of degree $d$ for some divisor $d$ of $2 n$. But for any $p$-group, $d$ is of the form $p^{k}(p-1)$ for some $k \geq 0$.

(c) Any characteristic subgroup $U$ of $O_{p}(G)$ is a normal subgroup of $G$. Thus by (a), the abelian group $U$ admits a faithful irreducible representation. Therefore $U$ is cyclic.

Suppose $N$ is a normal subgroup of an irreducible and symplectic primitive group $G<\mathrm{GL}_{2 n}(\mathbb{Q})$. Then the natural character $\chi$ of $N$ is sufficient to recover the conjugacy class of $N$. If $N$ has several $\mathbb{Q}$-irreducible faithfull representations, we will use the phrase " $G$ contains $N$ with character $\chi$ " to distinguish the conjugacy classes of matrix groups isomorphic to $N$.

If $\tilde{N}<\mathrm{GL}_{m}(\mathbb{Q})$ denotes an $\mathbb{Q}$-irreducible constituent of $N$, we will identify $N$ with $\tilde{N}$ since the precise notation $\tilde{N} \otimes I_{\frac{2 n}{m}}$ is not very handy.

The following theorem of Philip Hall classifies all finite $p$-groups whose abelian characteristic subgroups are cyclic. In particular, together with the above result, this classifies all possibible candidates for the Fitting subgroups of symplectic primitive irreducible maximal finite (s.p.i.m.f.) matrix groups. 
Theorem 2.1.17 (P. Hall) If $P$ is a finite p-group with no noncyclic abelian characteristic subgroups, then $P$ is the central product of subgroups $P_{1}$ and $P_{2}$ where

(a) $P_{1}$ is an extraspecial 2-group and $P_{2}$ is either a cyclic, dihedral, quasidihedral or generalized quaternion 2-group.

(b) $p$ is odd and $P_{1}$ is an extraspecial p-group of exponent $p$ and $P_{2}$ is cyclic.

Proof: See for example [Hup67, Satz 13.10, p. 357].

We close this section by showing that symplectic imprimitive matrix groups can easily be recognized. Further, the wreath products of symplectic primitive irreducible maximal finite (s.p.i.m.f.) matrix groups are usually again maximal finite symplectic. So we can restrict the classification to s.p.i.m.f. matrix groups.

Definition 2.1.18 Let $\mathcal{F}$ be a nonempty family of bilinear forms on $\mathbb{R}^{n}$. A lattice $L$ in $\mathbb{R}^{n}$ is called indecomposable w.r.t. $\mathcal{F}$, if $L$ cannot be written as a direct sum $L=L_{1} \oplus L_{2}$ where $b\left(L_{1}, L_{2}\right)=\{0\}$ for all $b \in \mathcal{F}$. A vector $x \in L$ is called indecomposable in $L$ w.r.t. $\mathcal{F}$ if it cannot be written as $x=y+z$ with $y, z \in L \backslash\{0\}$ and $b(y, z)=0$ for all $b \in \mathcal{F}$.

Theorem 2.1.19 Let $\mathcal{F}$ be a family of bilinear forms on $\mathbb{R}^{n}$ that contains at least one positive definite form $f$. Then each lattice $L$ in $\mathbb{R}^{n}$ admits a decomposition $L=\oplus_{i=1}^{k} L_{i}$ where each $L_{i}$ is indecomposable w.r.t. $\mathcal{F}$ and $b\left(L_{i}, L_{j}\right)=\{0\}$ for all $b \in \mathcal{F}$ and all $1 \leq i<j \leq k$. This decomposition is unique up to permutation of the $L_{i}$.

Proof: We adapt [Kne02, Satz (27.2)] slightly. Let $L=\oplus_{i=1}^{l} L_{i}^{\prime}$ be any decomposition such that $b\left(L_{i}^{\prime}, L_{j}^{\prime}\right)=0$ for all $b \in \mathcal{F}$ and all $i \neq j$. If $x \in L$ is indecomposable w.r.t. $\mathcal{F}$ then $x \in L_{i}^{\prime}$ for some $i$. Thus two indecomposable elements $x$ and $y$ with $b(x, y) \neq 0$ for some $b \in \mathcal{F}$ are in the same component $L_{i}^{\prime}$. Two indecomposable elements $x, y \in L$ are said to be equivalent if and only if there exists some indecomposable elements $x=x_{1}, \ldots, x_{r}=y \in L$ and some $b_{1}, \ldots, b_{r} \in \mathcal{F}$ such that $b_{i}\left(x_{i}, x_{i+1}\right) \neq 0$ for all $1 \leq i<r$. This defines an equivalence relation on the set of indecomposable elements of $L$. Since the equivalence classes give rise to a orthogonal decomposition of the Euclidean space $\left(\mathbb{R}^{n}, f\right)$ there are at most $n$ such classes $K_{1}, \ldots, K_{k}$ say. Denote by $L_{i}$ the sublattice of $L$ generated by $K_{i}$. For $1 \leq i<j \leq k$ we have $b\left(L_{i}, L_{j}\right)=\{0\}$ for all $b \in \mathcal{F}$ by construction. Further, every nonzero $x \in L$ can be written as a finite sum of indecomposable elements in $L$. If $x$ is decomposable, we find some $r, s \in L$ such that $x=r+s$ and $b(r, s)=0$ for all $b \in \mathcal{F}$. In particular $0<f(r, r), f(s, s)<f(x, x)$. Hence this decomposition procedure must end. Therefore $L=\oplus_{i=1}^{k} L_{i}$ is a decomposition of $L$ which has the desired properties. Each component $L_{i}$ is indecomposable and contained in $L_{j}^{\prime}$ for some $j$.

To proof the uniqueness, assume that all $L_{j}^{\prime}$ are also indecomposable. For $1 \leq j \leq l$ let $I_{j}=\left\{1 \leq i \leq k \mid L_{i} \subseteq L_{j}^{\prime}\right\}$ and set $M_{j}:=\oplus_{i \in I_{j}} L_{i} \subseteq L_{j}^{\prime}$. We are done if we can show $L_{j}^{\prime}=M_{j}$ for all $j$ since then $\left|I_{j}\right|=1$. Let $x \in L_{j}^{\prime}$. Write $x=\sum_{i=1}^{l} x_{i}$ with $x_{i} \in M_{i} \subseteq L_{i}^{\prime}$ for all $i$. Since $\oplus_{i=1}^{l} L_{i}^{\prime}=L$ this implies $x_{i}=0$ for all $i \neq j$. So $M_{j}=L_{j}^{\prime}$ as claimed. 
Remark 2.1.20 Let $G<\mathrm{GL}_{m}(\mathbb{Q})$ be finite and $L \in \mathcal{Z}(G)$. Then every automorphism in $\operatorname{Aut}(L, \mathcal{F}(G))$ permutes the components of the unique indecomposable orthogonal decomposition of $L$ wrt. $\mathcal{F}(G)$.

Hence a finite irreducible subgroup $G<\operatorname{Sp}_{2 n}(\mathbb{Q})$ is symplectic primitive if and only if each $L \in \mathcal{Z}(G)$ is indecomposable w.r.t. $\mathcal{F}(G)$.

Lemma 2.1.21 ([\le91, Proposition II.7]) Let $H<\mathrm{Sp}_{2 n}(\mathbb{Q})$ be s.p.i.m.f. such that $E:=\operatorname{End}(\bar{H})$ is a minimal totally complex number field. If the 2-modular trivial Brauer character is no constituent of the natural 2-modular character of $H$, then the wreath product $H<S_{k}<\operatorname{Sp}_{2 n k}(\mathbb{Q})$ is s.i.m.f. for all $k \geq 1$.

Proof: Since $-I_{n} \in H$ we have $\operatorname{End}\left(\overline{H \succ S_{k}}\right)=\left\{I_{k} \otimes c \mid c \in E\right\} \simeq E$ and $\mathcal{F}\left(H \curlyvee S_{k}\right)=$ $\left\{I_{k} \otimes F \mid F \in \mathcal{F}(H)\right\}$.

Let $L=L_{1} \oplus \cdots \oplus L_{k}$ for some $L_{i} \in \mathcal{Z}(G)$. View the $L_{i}$ as a $\tilde{H}$-module where $\tilde{H}$ is the direct product of $k$ copies of $H$. By our assumption $L_{i}$ and $L_{j}$ have no common $p$-modular constituent for $i \neq j$ as $\tilde{H}$-modules. By [Ple78, Theorem I.1] we get $\mathcal{Z}(\tilde{H})=\left\{\oplus_{i=1}^{k} L_{i} \mid L_{i} \in \mathcal{Z}(H)\right\}$. Hence $\mathcal{Z}\left(H<S_{k}\right)=\left\{\oplus_{i=1}^{k} L \mid L \in \mathcal{Z}(H)\right\}$. The result now follows since $E \simeq \operatorname{End}\left(\overline{H \succ S_{k}}\right)$ is minimal totally complex and $H \succ S_{k}=$ $\operatorname{Aut}\left(L, \mathcal{F}\left(H \prec S_{k}\right)\right)$ for all $L \in \mathcal{Z}\left(H \succ S_{k}\right)$.

The assumption on the 2-modular constituents is necessary. The group $H:=$ $\left\langle\left(\begin{array}{cc}0 & 1 \\ -1 & 0\end{array}\right)\right\rangle<\mathrm{Sp}_{2}(\mathbb{Q})$ is s.p.i.m.f. but $H \curlyvee S_{2} \leq \mathrm{Sp}_{4}(\mathbb{Q})$ is not maximal finite (see Theorem 4.3.1). In fact, this is the only example that we will encounter.

\subsubsection{Generalized Bravais groups}

If $N$ is a normal subgroup of a s.p.i.m.f. matrix group $G$ then

$$
\mathcal{B}^{o}(N):=\{x \in G \mid x \text { centralizes } \operatorname{End}(\bar{N})\}
$$

is also a normal subgroup of $G$ which contains $N$. We will show that $\mathcal{B}^{\circ}(N)$ can be computed from the group $N$ without knowledge of $G$.

The natural $\bar{N}$-module $\mathbb{Q}^{1 \times m}$ decomposes into a direct sum of copies of a single irreducible $\bar{N}$-module $V$ as we have seen in Corollary 2.1.16.

The $\mathbb{Z}$-span $\Lambda_{1}(N):=\langle N\rangle_{\mathbb{Z}}$ is invariant under conjugation by $G$ and we recursively define an ascending chain of orders in $\bar{N}$ having the same property. This is the so called radical idealizer process:

- If $\Lambda_{i}(N)$ has already been defined, then let $R_{i}$ be the arithmetic radical of $\Lambda_{i}(N)$ i.e. the intersection of all maximal ideals of $\Lambda_{i}(N)$ that contain the (reduced) discriminant of $\Lambda_{i}(N)$.

- Let $\Lambda_{i+1}(N)$ be the right order of $R_{i}$ in $\bar{N}$. 
If $\Lambda_{i}(N)$ is $G$-invariant then $R_{i}$ is also $G$-invariant by definition. So $g \in G$ and $x \in \Lambda_{i+1}(N)$ imply $R_{i}\left(g^{-1} x g\right)=g^{-1} R_{i} x g \subseteq R_{i}$. This shows that $\Lambda_{i+1}(N)$ is $G$ invariant as claimed.

Like any ascending chain of orders having full rank in $\bar{N}$, this chain stabilizes at some order $\Lambda_{\infty}(N)$ say. It follows from [Rei03, Theorems (39.11), (39.14) and (40.5)] that the above chain stabilizes at $\Lambda_{i}(N)$ if and only if $\Lambda_{i}(N)$ is hereditary. So in particular $\Lambda_{\infty}(N)$ is hereditary.

Since $\Lambda_{\infty}(N)$ is $G$-invariant, $G$ acts on $\mathcal{Z}\left(\Lambda_{\infty}(N)\right)$. Thus $G$ fixes at least one of the $\Lambda_{\infty}(N)$-lattices. This leads to the following definition.

Definition 2.1.22 With the above notation, let $F \in \mathcal{F}_{>0}(N)$. The generalized Bravais group of $N$ is

$$
\mathcal{B}^{o}(N):=\left\{g \in \bar{N} \mid L_{i} g=L_{i} \text { for all } 1 \leq i \leq s \text { and } g F g^{\text {tr }}=F\right\}
$$

where $\left\{L_{1}, \ldots, L_{s}\right\}$ represents the isomorphism classes of $\Lambda_{\infty}(N)$-lattices in $V$.

By construction $\mathcal{B}^{\circ}(N)$ is a finite subgroup of $\bar{N}^{*}$ containing $N$. Moreover $N$ and $\mathcal{B}^{\circ}(N)$ have the same commuting algebras and thus the same invariant forms. In particular, $\mathcal{B}^{\circ}(N)$ does not depend on the choice of $F$.

Lemma 2.1.23 Let $G<\operatorname{Sp}_{2 n}(\mathbb{Q})$ be s.p.i.m.f.. If $N \unlhd G$ then

(a) $N \unlhd \mathcal{B}^{o}(N) \unlhd G$

(b) If $X<\bar{N}^{*}$ is a finite subgroup such that $N \unlhd X$ then $X \leq \mathcal{B}^{\circ}(N)$.

(c) $\mathcal{B}^{o}(N)=\{x \in G \mid x$ centralizes $\operatorname{End}(\bar{N})\}$

Proof: The parts (a) and (b) are proven in [NP95, Proposition II.10, p. 82]. Part (c) follows from (a), (b) and the double centralizing property.

Part (b) can be used to find a large subgroup of $\mathcal{B}^{\circ}(N)$. Part (a) rules out some normal subgroups. For example, suppose $G<\mathrm{Sp}_{10 k}$ is s.p.i.m.f. such that $G$ contains a normal subgroup $N \simeq$ Alt $_{5}$ where the character of $N$ is a multiple of the 5 -dimensional irreducible character of $\mathrm{Alt}_{5}$. Then (by Table 2.5.1) $N \not \mathcal{B}^{\circ}(N) \simeq C_{2} \times \mathrm{Alt}_{6}$ gives a contradiction. 


\subsubsection{General outline of the classification}

We recall the definition of the generalized Fitting subgroup and its self-centralizing property. In this section, let $G$ be a finite group.

Definition 2.1.24 A finite perfect group $H$ is called quasisimple if $H / Z(H)$ is simple. A component of $G$ is a subnormal quasisimple subgroup of $G$. The subgroup generated by all components of $G$ is called the layer of $G$ and is denoted by $E(G)$. Finally, the generalized Fitting subgroup $F^{*}(G)$ of $G$ is the subgroup generated by the layer $E(G)$ and the Fitting subgroup $F(G)=\prod_{p \| G \mid} O_{p}(G)$.

Lemma 2.1.25 The layer $E(G)$ is a central product of its components. The generalized Fitting subgroup $F^{*}(G)$ is the central product of $F(G)$ and $E(G)$.

Proof: See for example [Asc00, 31.7 and 31.12].

Theorem 2.1.26 The generalized Fitting subgroup $F^{*}(G)$ is self-centralizing in $G$. In particular, $G / F^{*}(G)$ is isomorphic to a subgroup of $\operatorname{Out}\left(F^{*}(G)\right)$.

Proof: See for example [Asc00, 31.13].

Remark 2.1.27 The general outline of the classification of the s.i.m.f. subgroups of $\mathrm{Sp}_{2 n}(\mathbb{Q})$ is now as follows:

(a) The symplectic imprimitive matrix groups come from the classifications of $\operatorname{Sp}_{2 d}(\mathbb{Q})$ where $d$ runs through all divisors of $n$. These groups are usually s.i.m.f. (see Lemma 2.1.21). Suppose now $G<\mathrm{Sp}_{2 n}(\mathbb{Q})$ is s.p.i.m.f..

(b) There are only finitely many candidates for the Fitting subgroup $F(G)$ according to Theorem 2.1.17. These are listed in Table 2.5.2.

(c) There are only finitely many candidates for the layer $E(G)$. These are described in [HM01] which is based on the ATLAS [CCN ${ }^{+}$85] and listed in Table 2.5.1 up to $n=11$. (Note that this step depends on the completeness of the classification of all finite simple groups).

(d) For two such candidates $F(G)$ and $E(G)$ we know that $G / F^{*}(G)$ is isomorphic to a subgroup of $\operatorname{Out}\left(F^{*}(G)\right)$. So we have to find all such possible extensions of $F^{*}(G)$ up to conjugacy in $\mathrm{GL}_{2 n}(\mathbb{Q})$.

The last step is the crucial one. Although it is a cohomological task to find all abstract extensions $G$, we are interested in finding all matrix group extension of $F^{*}(G)$. Of course, one can always replace $F^{*}(G)$ by its generalized Bravais group.

In the next section, we will describe methods that construct $G$ from $F^{*}(G)$ under certain assumptions on $F^{*}(G)$. We will also give some more criteria that eliminate some candidates for $F^{*}(G)$. 


\subsection{Methods}

\subsubsection{Normal subgroups of index $2^{k}$}

For many s.i.m.f. matrix groups $G$ the quotient $G / \mathcal{B}^{\circ}\left(F^{*}(G)\right)$ is an elementary abelian 2-group as Tables 2.5.2 and 2.5.1 show. Thus we give two results that rule out some candidates for normal subgroups $N$ having index $2^{k}$ in $G$.

Part (c) of the next lemma is an analogon to [NP95, Corollary III.4]. It will be used frequently in the classification.

Lemma 2.2.1 Let $N \triangleleft G$ with $[G: N]=2$ where $G<\operatorname{Sp}_{2 n}(\mathbb{Q})$ is irreducible and symplectic primitive. Let $g \in G \backslash N$ and suppose that $N$ is reducible in $\mathrm{GL}_{2 n}(\mathbb{Q})$.

(a) The restriction $\left.\triangle\right|_{N}$ of the natural representation of $G$ onto $N$ splits into $\triangle_{1}+\triangle_{1}^{g}$ where $\triangle_{1}: N \rightarrow \mathrm{GL}_{n}(\mathbb{Q})$ is irreducible.

(b) $\operatorname{dim}_{\mathbb{Q}} \mathcal{F}_{\text {skew }}\left(\triangle_{1}(N)\right)=0$.

(c) $L:=\operatorname{End}\left(\overline{\triangle_{1}(N)}\right) \subseteq \mathbb{Q}^{n \times n}$ is a totally real number field.

Proof: (a) By Clifford theory.

(b) If $\operatorname{dim}_{\mathbb{Q}} \mathcal{F}_{\text {skew }}\left(\triangle_{1}(N)\right)>0$ then all nonzero elements in $\mathcal{F}_{\text {skew }}\left(\triangle_{1}(N)\right)$ are invertible, since $\triangle_{1}$ is irreducible. Thus $\triangle_{1}(N)$ is symplectic. The representation $\triangle$ is induced by $\triangle_{1}$, hence we may suppose that $\triangle(N)$ is given by blockdiagonal matrices and $\triangle(g)=\left(\begin{array}{cc}0 & I_{n} \\ \triangle_{1}\left(g^{2}\right) & 0\end{array}\right)$. But then $G \leq \triangle_{1}(N) \prec C_{2}$ is symplectic imprimitive.

(c) $\triangle_{1}$ can also be seen as a real representation $\delta: N \rightarrow \mathrm{GL}_{n}(\mathbb{R})$. Let $\delta$ decompose into $\mathbb{R}$-irreducible representations $\delta_{1}, \ldots, \delta_{s}$ say. Then $\operatorname{dim}_{\mathbb{R}} \mathcal{F}_{\text {skew }}(\delta(N))=$ $\operatorname{dim}_{\mathbb{Q}} \mathcal{F}_{\text {skew }}\left(\triangle_{1}(N)\right)=0$ and hence $\operatorname{dim}_{\mathbb{R}} \mathcal{F}_{\text {skew }}\left(\delta_{i}(N)\right)=0$ for all $i$.

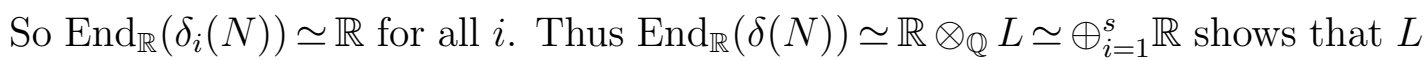
must be a ring direct sum of totally real number fields. Since $L$ is simple, the result follows.

Lemma 2.2.2 Let $A$ be a simple $\mathbb{Q}$-algebra. Suppose that $\alpha \in \operatorname{Aut}_{\mathbb{Q}}(A)$ is a $\mathbb{Q}$ algebra automorphism of order 2 . If $A^{+}:=\{x \in A \mid \alpha(x)=x\}$ is a simple ring, then $\operatorname{dim}_{\mathbb{Q}}(A)=2 \operatorname{dim}_{\mathbb{Q}}\left(A^{+}\right)$.

Proof: Let $A^{-}=\{x \in A \mid \alpha(x)=-x\}$. Then $A=A^{+} \oplus A^{-}$. The automorphism $\alpha$ maps the center $K$ of $A$ onto itself and hence induces an automorphism of $K$. If $\left.\alpha\right|_{K}$ is not trivial, there exists some $x \in K$ such that $x \neq \alpha(x)$. But then $a:=x-\alpha(x) \in$ $K^{*} \cap A^{-}$. In particular, $A^{+} \rightarrow A^{-}, x \mapsto a x$ is an isomorphism of $\mathbb{Q}$-spaces and the result follows.

So we may now suppose that $\alpha \in \operatorname{Aut}_{K}(A)$. In particular, $A^{+}$is a central simple $K$-algebra and by Skolem-Noether, $\alpha$ is conjugation by some invertible $g \in A^{+}$. The 
Wedderburn theorem allows us to identify $A$ with $\mathcal{Q}^{n \times n}$ for some skewfield $\mathcal{Q}$ with center $K$. Let $s:=g^{2} \in K$. If $s \in K^{2}$, then we may assume that $g^{2}=1$ thus $g$ is conjugate to $\operatorname{Diag}\left(I_{k},-I_{l}\right)$ in $K^{n \times n}$ for some $k+l=n$. But then $A^{+}=\mathcal{Q}^{k \times k} \oplus \mathcal{Q}^{l \times l}$ is simple if and only if $g$ is central. This contradicts the assumptions. So $\tilde{K}:=K(\sqrt{s})$ is a proper extension of $K$. Let $\tilde{g}:=\frac{1}{\sqrt{s}} g$ and $\tilde{A}:=A \otimes_{K} \tilde{K}$. Again, since $\tilde{A}$ is a central simple $\tilde{K}$-algebra, we may assume that $\tilde{A}=\tilde{Q}^{\tilde{n} \times \tilde{n}}$ for some skewfield $\tilde{Q}$ with center $\tilde{K}$. As above $\tilde{g}:=\frac{1}{\sqrt{s}} g$ is conjugate to $\operatorname{Diag}\left(I_{k},-I_{l}\right)$ in $\tilde{K}^{\tilde{n} \times \tilde{n}}$ with $k+l=\tilde{n}$. Thus $\tilde{A}^{+}=C_{\tilde{A}}(g)=\tilde{\mathcal{Q}}^{k \times k} \oplus \tilde{\mathcal{Q}}^{l \times l}$. But on the other hand, $e:=\frac{1}{2}(1+\tilde{g})$ and $f:=\frac{1}{2}(1-\tilde{g})$ are (the unique) central primitive idempotents of $\tilde{A}^{+}$. Let $\langle\sigma\rangle=\operatorname{Gal}(\tilde{K} / K) \simeq C_{2}$. By acting on the structure constants of $\tilde{A}^{+}, \sigma$ extends to an $K$-algebra automorphism of $\tilde{A}^{+}$such that $\sigma(\sqrt{s})=-\sqrt{s}$ and $\sigma(x)=x$ for all $x \in A^{+}$. In particular $\sigma(e)=f$. Thus $\sigma$ interchanges the ring direct summands $\tilde{\mathcal{Q}}^{k \times k}$ and $\tilde{\mathcal{Q}}^{l \times l}$. This shows $k=l=\frac{\tilde{n}}{2}$ and the result follows, since $\operatorname{dim}_{K}\left(A^{+}\right)=\operatorname{dim}_{\tilde{K}}\left(\tilde{A}^{+}\right)=\frac{\tilde{n}^{2}}{2} \cdot \operatorname{dim}_{\tilde{K}}(\tilde{Q})=\frac{1}{2} \operatorname{dim}_{\tilde{K}}(\tilde{A})=$ $\frac{1}{2} \operatorname{dim}_{K}(A)$.

If $n$ is not a power of 2 , the following corollary is used to rule out some candidates for normal subgroups. See [Neb95, (III.4)] for a similar result in $\mathrm{GL}_{n}(\mathbb{Q})$.

Corollary 2.2.3 Let $G<\operatorname{Sp}_{2 n}(\mathbb{Q})$ be irreducible and symplectic primitive. If $N \triangleleft G$ with $|G / N|=2^{k}$, then $\operatorname{dim}_{\mathbb{Q}}(\operatorname{End}(\bar{G}))=2^{l} \operatorname{dim}_{\mathbb{Q}}(\operatorname{End}(\bar{N}))$ for some $0 \leq l \leq k$.

Proof: Let $N=N_{1} \triangleleft N_{2} \triangleleft \ldots N_{r-1} \triangleleft N_{r}=G$ be a normal series of $G$ such that $N_{i+1} / N_{i} \simeq C_{2}$. By Corollary 2.1.16 all the commuting algebras $E_{i}:=\operatorname{End}\left(\overline{N_{i}}\right)$ are simple. Let $g \in N_{2} \backslash N_{1}$. Then $g$ induces an automorphism on $E_{2}$ of order at most 2 . So by the result above, $E_{2}=C_{E_{1}}(g)$ either equals $E_{2}$ or has dimension $\frac{1}{2} \operatorname{dim}_{\mathbb{Q}}\left(E_{1}\right)$. The result follows by induction.

\subsubsection{Primitive, normalized and normal critical lattices}

Suppose $G<\mathrm{GL}_{m}(\mathbb{Q})$. To find the r.i.m.f. or s.i.m.f. supergroups of $G$, one has to consider automorphism groups of $(L, F) \in \mathcal{Z}(G) \times \mathcal{F}(G)$. Since the number of such pairs $(L, F)$ is infinite, we have to reduce this number. In this section we start with some general results that do not depend on $\operatorname{End}(\bar{G})$.

The following definitions will be used frequently in the sequel.

Definition 2.2.4 Let $L^{\prime}, L \subset \mathbb{Q}^{1 \times m}$ be $\mathbb{Z}$-lattices of full rank and let $F^{\prime}, F \in \mathbb{Q}^{m \times m}$ be symmetric and positive definite.

(a) $L^{\#, F}:=\left\{x \in \mathbb{Q}^{1 \times m} \mid x F y^{\text {tr }} \in \mathbb{Z}\right.$ for all $\left.y \in L\right\}$ is the dual lattice of $L$ wrt. $F$.

(b) $F$ is said to be integral on $L$ if $L \subseteq L^{\#, F}$. 
(c) $F$ is said to be primitive on $L$ if $L \subseteq L^{\#, F}$ but $L \nsubseteq k L^{\#, F}$ for all $k \in \mathbb{Z}_{>1}$.

(d) $(L, F)$ is normalized if $L \subseteq L^{\#, F}$ and the abelian group $L^{\#, F} / L$ is of squarefree exponent with rank at most $\frac{m}{2}$.

(e) The pairs $(L, F)$ and $\left(L^{\prime}, F^{\prime}\right)$ are said to be isometric if there exists some $x \in$ $\mathrm{GL}_{m}(\mathbb{Q})$ such that $L^{\prime}=L x$ and $F^{\prime}=x^{-1} F x^{-\operatorname{tr}}$. In this case $x$ is called an isometry between $L$ and $L^{\prime}$.

The next remark shows that every finite matrix group $G$ fixes a normalized pair $(L, F)$.

Remark 2.2.5 Let $G<\mathrm{GL}_{m}(\mathbb{Q})$ be finite.

(a) The set $\mathcal{Z}(G)$ is closed under the following operations

(1) $\mathcal{Z}(G) \times \mathcal{F}_{>0}(G) \rightarrow \mathcal{Z}(G),(L, F) \mapsto L^{\#, F}$

(2) $\mathcal{Z}(G) \times \operatorname{End}(\bar{G})^{*} \rightarrow \mathcal{Z}(G),(L, c) \mapsto L c$

(3) $\mathcal{Z}(G) \times \mathcal{Z}(G) \rightarrow \mathcal{Z}(G),\left(L, L^{\prime}\right) \mapsto L+L^{\prime}$

(4) $\mathcal{Z}(G) \times \mathcal{Z}(G) \rightarrow \mathcal{Z}(G),\left(L, L^{\prime}\right) \mapsto L \cap L^{\prime}$

(5) $\mathcal{Z}(G) \times N_{\mathrm{GL}_{m}(\mathbb{Q})}(G) \rightarrow \mathcal{Z}(G),(L, h) \mapsto L h$

(b) Let $F \in \mathcal{F}_{>0}(G)$ be integral on $L \in \mathcal{Z}(G)$. If $(L, F)$ is not normalized, then there exist some prime divisor $p$ of $\operatorname{det}(L, F)$ such that $\left(L \cap p L^{\#, F}, \frac{1}{p} F\right)$ is an integral lattice of smaller determinant. In particular, iterating this process results in some normalized $\left(L^{\prime}, \frac{1}{d} F\right) \in \mathcal{Z}(G) \times \mathcal{F}_{>0}(G)$ with $d \mid \operatorname{det}(L, F)$.

Now we want to reduce the number of lattices $L$ that we have to consider.

Remark 2.2.6 If $\Lambda$ is a $\mathbb{Z}$-order in $\mathbb{Q}^{m \times m}$ then $L, L^{\prime} \in \mathcal{Z}(\Lambda)$ are isomorphic as $\Lambda$ right modules if and only if there exists some $x \in C_{\mathbb{Q}^{m \times m}}(\Lambda)$ such that $L x=L^{\prime}$. The number of isomorphism classes is finite by the Jordan-Zassenhaus theorem (see [Rei03, Chapter 26]).

Let $G<\mathrm{GL}_{m}(\mathbb{Q})$ be finite. Then $(L, F) \in \mathcal{Z}(G) \times \mathcal{F}(G)$ is isometric to $\left(L x, x^{-1} F x^{- \text {tr }}\right)$ for all $x \in \operatorname{End}(\bar{G})^{*}$. Since isometric pairs have conjugate automorphism groups, it suffices to consider pairs $(L, F)$ where $L$ runs through a (finite) system of representatives of the isomorphism classes of $\mathcal{Z}(G)$.

If one wants to find all r.i.m.f. or s.p.i.m.f. groups that contain $G$ as a normal subgroup, one can usually reduce the number of lattices $L$ that one has to consider even further.

Definition 2.2.7 Let $G<\mathrm{GL}_{m}(\mathbb{Q})$ be finite. A finite subset $S \subset \mathcal{Z}(G)$ is called $G$-normal critical, if for every finite supergroup $H<\mathrm{GL}_{m}(\mathbb{Q})$ with $G \unlhd H$ there exists some $x \in \mathrm{GL}_{m}(\mathbb{Q})$ such that $\mathcal{Z}\left(H^{x}\right) \cap S \neq \emptyset$. If $S=\{L\}$, then $L$ is called $G$-normal critical. 
Remark 2.2.8 Let $G<\mathrm{GL}_{m}(\mathbb{Q})$ be finite such that $E:=\operatorname{End}(\bar{G})$ is a field. When one wants to find a set of $G$-normal critical lattices, one usually has to deal with one of the following situations:

Let $\Lambda:=\left\langle G, \mathbb{Z}_{E}\right\rangle_{\mathbb{Z}}$. The fractional $\mathbb{Z}_{E}$-ideals act on $\mathcal{Z}(\Lambda)$. Let $L_{1}, \ldots, L_{s}$ represent the orbits.

(a) If there exists some $1 \leq i \leq s$ such that every $h \in N_{\mathrm{GL}_{m}(\mathbb{Q})}(G)$ of finite order fixes the set $\mathcal{L}_{i}:=\left\{L_{i} \mathfrak{a} \mid \mathfrak{a}\right.$ a fractional $\mathbb{Z}_{E^{-}}$-ideal $\}$then $\mathcal{S}=\left\{L_{i} \mathfrak{a} \mid[\mathfrak{a}] \in \mathcal{C} l\left(\mathbb{Z}_{E}\right)\right\}$ is $G$-normal critical.

This situation can arise as follows:

(1) Suppose there exists some $1 \leq i \leq s$ such that for all $j \neq i$ and all fractional ideals $\mathfrak{a}$ of $\mathbb{Z}_{E}$ the determinant of a base change matrix from $L_{i}$ to $L_{j} \mathfrak{a}$ does not equal \pm 1 . Then $\mathcal{L}_{i}$ has the above property.

(2) Choose one of the following options to definite $m_{i}$ and $M_{i}$ for $1 \leq i \leq s$ :

- Let $\Lambda^{\prime}$ be either $\Lambda$ or $\langle G\rangle_{\mathbb{Z}}$. Let $m_{i}$ be the number of minimal $\Lambda^{\prime}$ invariant sublattices of $L_{i}$ whose index in $L_{i}$ has only prime divisors in a given fixed set.

- Let $m_{i}=\mid\left\{L \in \mathcal{Z}(G) \backslash \mathcal{Z}(\Lambda) \mid L\right.$ a minimal sublattice of $\left.L_{i}\right\} \mid$.

Similarly one defines $M_{1}, \ldots, M_{s}$ by taking superlattices in the definitions above. If there exists some $1 \leq i \leq s$ such that $\left(m_{i}, M_{i}\right) \neq\left(m_{j}, M_{j}\right)$ for all $j \neq i$ then $\mathcal{L}_{i}$ has the above property.

(b) Suppose that $s=4$ and suppose that the $L_{i}$ can be chosen such that $L_{1}=L_{2}+L_{3}$, $L_{4}=L_{2} \cap L_{3}$ and there are no elements of $\mathcal{Z}(\Lambda)$ between $L_{1} / L_{i}, L_{i} / L_{4}$ for $i=2,3$. Then $\mathcal{S}=\left\{L_{i} \mathfrak{a} \mid i \in\{1,2\},[\mathfrak{a}] \in \mathcal{C} l\left(\mathbb{Z}_{E}\right)\right\}$ is a $G$-normal critical set.

Proof: Let $H<N_{\mathrm{GL}_{m}(\mathbb{Q})}(G)$ be finite.

(a) Let $O:=L_{i} \cdot H$ be the orbit of $L_{i}$ under the action of $H$. Then $L:=\sum_{L^{\prime} \in O} L^{\prime} \in$ $\mathcal{Z}(\Lambda)$. By the assumption, $O \subset \mathcal{L}_{i}$. So for each $L^{\prime} \in O$ there exists a fractional $\mathbb{Z}_{E^{\text {-ideal }}} \mathfrak{a}_{L^{\prime}}$ such that $L^{\prime}=L_{i} \mathfrak{a}_{L^{\prime}}$. But then $L=L_{i} \mathfrak{a}$ for $\mathfrak{a}=\sum_{L^{\prime} \in O} \mathfrak{a}_{L^{\prime}}$. So $\mathcal{S}$ is $G$-normal critical.

Part (a1) is obvious since $\operatorname{det}(h) \in\{ \pm 1\}$ for all $h \in H$ and (a2) follows from the fact that the action of $H$ on $\mathcal{Z}(G)$ preserves inclusions, endomorphism rings and the index of sublattices.

(b) Summing over $H$ shows that $H$ fixes $L_{i} \mathfrak{a}$ for some fractional $\mathbb{Z}_{E}$-ideal $\mathfrak{a}$ and some $1 \leq i \leq 4$. If $i=4$ then $H$ fixes $L_{1} \mathfrak{a}=L_{2} \mathfrak{a}+L_{3} \mathfrak{a}$ since $L_{2} \mathfrak{a}$ and $L_{3} \mathfrak{a}$ are the unique minimal superlattices of $L_{4} \mathfrak{a}$ in $\mathcal{Z}(\Lambda)$ which are not of the form $L_{4} \mathfrak{a}^{\prime}$ for

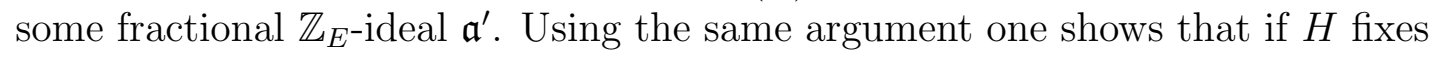
$L_{3} \mathfrak{a}$ then it also fixes $L_{2} \mathfrak{a}$. 


\subsubsection{Fields as endomorphism rings (m-parameter argument)}

In this section, we give an algorithm (the so called $m$-parameter argument) that constructs all r.i.m.f. or s.i.m.f. supergroups $G$ of an irreducible matrix group $U$ if $\operatorname{End}(\bar{U})$ is a field. In particular, this includes irreducible cyclic matrix groups $U$.

By the previous section, the problem is to reduce the number of forms that one has to consider. To do so, we need all possible prime divisors of $|G|$. If we have no other assumptions on $G$, we can always fall back on the Minkowski bound:

Lemma 2.2.9 (Minkowski's bound, [Min87]) The least common multiple of the orders of all finite subgroups of $\mathrm{GL}_{n}(\mathbb{Q})$ is given by

$$
\prod_{p} p^{\left\lfloor\frac{n}{(p-1)}\right\rfloor+\left\lfloor\frac{n}{p(p-1)}\right\rfloor+\left\lfloor\frac{n}{p^{2}(p-1)}\right\rfloor+\ldots}
$$

where the product is taken over all primes $p \leq n+1$.

Further, the $m$-parameter argument needs a set of primes $\tilde{\Pi}(\operatorname{End}(\bar{U}),|G|)$ depending on $\operatorname{End}(\bar{U})$ and $|G|$ as follows:

Definition 2.2.10 Let $K$ be an algebraic number field.

(a) Let $\sigma_{i}: K \rightarrow \mathbb{R} \quad(1 \leq i \leq d)$ be the real embeddings of $K$. If we fix the order of the $\sigma_{i}$, we get a group homomorphism

$$
s: K^{*} \rightarrow \mathbb{F}_{2}^{m}, \quad x \mapsto\left(x_{1}, \ldots, x_{d}\right) \text { where } x_{i}=\left\{\begin{array}{ll}
0 & \text { if } \sigma_{i}(x)>0, \\
1 & \text { if } \sigma_{i}(x)<0
\end{array} .\right.
$$

(b) For $k \in \mathbb{Z}$ define $\Pi(k)$ to be the set of all primes dividing $k$.

(c) We define a finite set of primes $\Pi(K)$ such that

(1) In each class of $\mathcal{C l}\left(\mathbb{Z}_{K}\right)$ there exists an integral ideal which contains $\prod_{p \in \Pi(K)} p^{a_{p}}$ with some $a_{p} \in \mathbb{N}_{0}$.

(2) The group $s\left(K^{*}\right)$ is generated by $s\left(x_{1}\right), \ldots, s\left(x_{\ell}\right)$ for some $x_{i} \in \mathbb{Z}_{K}$ satisfying $\Pi\left(\mathrm{Nr}_{K / \mathbb{Q}}\left(x_{i}\right)\right) \subseteq \Pi(K)$.

(d) For $k \in \mathbb{Z}$ set $\tilde{\Pi}(K, k):=\Pi(k) \cup \bigcup_{F \leq K} \Pi(F)$ where the union is taken over all subfields $F$ of $K$. Note that this set is not unique.

We follow [Neb95, Satz III.2, p. 16] to give an algorithm which constructs all r.i.m.f. or s.i.m.f. supergroups of a given irreducible matrix group of the same dimension. 
Theorem 2.2.11 Let $G<\mathrm{GL}_{n}(\mathbb{Q})$ be finite and irreducible. Let $L$ be a $\mathbb{Z} G$-lattice. Assume that $C:=C_{\mathbb{Q}^{n \times n}}(G)$ is either commutative or a positive definite quaternion algebra. Then there exists a $F \in \mathcal{F}_{>0}(G)$ such that $F$ is primitive on $L$ and $\Pi(\operatorname{det}(L, F)) \subseteq \Pi(K) \cup \Pi(|G|)$ where $K$ denotes the maximal real subfield of $Z(C)$.

Proof: If $C$ is commutative, a proof is given in [Neb95, Satz III.2, p. 16]. So we may assume that $C$ is a positive definite quaternion algebra. Choose any $F \in \mathcal{F}_{>0}(G)$ which is primitive on $L$. Suppose that there exists some prime $p \notin \Pi(K)$ such that $p \mid \operatorname{det}(L, F)$ but $p \nmid|G|$. It suffices to show that there exists some $c \in K$ such that $c F$ is integral on $L$ and the primes dividing $\operatorname{det}(L, c F)$ are contained in $(\Pi(|G|$. $\operatorname{det}(L, F)) \cup \Pi(K))) \backslash\{p\}$.

Let $\mathfrak{p}_{1}, \ldots, \mathfrak{p}_{\ell}$ be the prime ideals of $\mathbb{Z}_{K}$ over $p$. Then $K_{p}:=K \otimes_{\mathbb{Q}} \mathbb{Q}_{p}=\bigoplus_{i=1}^{\ell} K_{\mathfrak{p}_{i}}$. Denote by $\varepsilon_{1}, \ldots, \varepsilon_{\ell}$ the primitive Idempotents of $K_{p}$ such that $K_{p} \varepsilon_{i}=K_{\mathfrak{p}_{i}}$.

Let $L_{p}:=L \otimes_{\mathbb{Z}} \mathbb{Z}_{p}$ and $C_{p}:=C \otimes_{\mathbb{Q}} \mathbb{Q}_{p}$. Since $p \nmid|G|, p$ is not ramified in $\bar{G}$ and the order $\Lambda_{p}:=\langle G\rangle_{\mathbb{Z}_{p}}$ is maximal in $(\bar{G})_{p}$ (see [Rei03, Theorems 41.1 and 41.7]). Therefore $\operatorname{End}_{\Lambda_{p}}\left(L_{p}\right)$ is maximal in $C_{p}$ and $p$ is not ramified in $C_{p}$. Hence $C_{p} \simeq \bigoplus_{i=1}^{\ell} K_{\mathfrak{p}_{i}}^{2 \times 2}$.

So each $\Lambda_{p}$-lattice $L_{p} \varepsilon_{i}$ decomposes into two irreducible $\Lambda_{p}$-lattices $X_{i, 1} \oplus X_{i, 2}$. From $\varepsilon_{i} \in K \subseteq Z(C)$ we get $\varepsilon_{i} F\left(1-\varepsilon_{i}\right)^{t r}=0$ which shows that the lattices $L_{p} \varepsilon_{i}$ are orthogonal to each other.

Since $\operatorname{End}_{K_{\mathfrak{p}_{i}} G}\left(X_{i, 1}\right) \simeq K_{\mathfrak{p}_{i}}$, there exists no symmetric $G$-invariant nonzero form on $X_{i, 1}$. Hence $F$ induces an embedding $\varphi_{i}: X_{i, 1} \hookrightarrow X_{i, 2}{ }^{*}, x \mapsto F x^{t r}$ for each $i$. So there exists some $k_{i} \in \mathbb{Z}$ such that $\varphi_{i}\left(X_{i, 1}\right)=p^{k_{i}} \cdot X_{i, 2}{ }^{*}$. Therefore the Gram matrix of $\left(L_{p}, F\right)$ is of the form $\operatorname{diag}\left(p^{k_{1}} G_{1}, \ldots, p^{k_{\ell}} G_{\ell}\right)$ for some $G_{i} \in \mathrm{GL}_{n_{i}}\left(\mathbb{Z}_{p}\right)$ (wrt. a proper choice of a basis).

By property (1) of the definition of $\Pi(K)$, there exists some $\mathbb{Z}_{K}$-ideal $\mathfrak{a}_{i}$ whose norm is only divisible by primes in $\Pi(K)$ and some $y_{i} \in K$ such that $\mathfrak{p}_{i} \cdot \mathfrak{a}_{i}=y_{i} \mathbb{Z}_{K}$. Then $\mathfrak{p}_{i}=\left\langle p, y_{i}\right\rangle$ since this identity holds locally everywhere. In particular $y_{i} \in \mathbb{Z}_{K}$ and $\Pi\left(\mathrm{Nr}_{K / \mathbb{Q}}\left(y_{i}\right)\right) \subseteq\{p\} \cup \Pi(K)$. By property (2) of the definition of $\Pi(K)$, there exists some $x \in K$ such that $\operatorname{Nr}_{K / \mathbb{Q}}(x) \in \Pi(K)$ and $y:=x \cdot \prod_{i=1}^{\ell} y_{i}^{-k_{i}}$ is totally positive.

Let $F^{\prime}:=y \cdot F$. Then $y_{i} \varepsilon_{i}$ and $p \varepsilon_{i}$ are both primitive elements of $K_{\mathfrak{p}_{i}}$. Hence $\left(L_{p}, F^{\prime}\right)$ is self-dual. It might happen that $F^{\prime}$ is no longer integral on $L$. But then there exists some $k \in \mathbb{N}$ such that $k F^{\prime}$ is primitive on $L$ and the primes dividing $k$ are divisors of $|G|$.

From this theorem we finally obtain

Corollary 2.2.12 (m-parameter argument, [Neb95, Korollar III.3, p. 17]) Let $U \leq G<\mathrm{GL}_{n}(\mathbb{Q})$ be finite subgroups such that $C:=\operatorname{End}(\bar{U})$ is a either a field or a positive definite quaternion algebra. Suppose $L \in \mathcal{Z}(G) \subseteq \mathcal{Z}(U)$. Then there exists some $F \in \mathcal{F}_{>0}(G)$ that is primitive on $L$ with $\Pi(\operatorname{det}(L, F)) \subseteq \tilde{\Pi}(K,|G|)$ where $K$ denotes the maximal real subfield of the center of $C$.

Proof: The maximal totally real subfield $K^{\prime}$ of the center of $\operatorname{End}(\bar{G})$ is contained in $K$. By the theorem above, there exists some $F \in \mathcal{F}_{>0}(G)$ such that $F$ is primitive on $L$ and $\Pi(\operatorname{det}(L, F)) \subseteq \Pi\left(K^{\prime},|G|\right) \subseteq \tilde{\Pi}(K,|G|)$. 
The following rather technical remark shows how this corollary will be used later.

Remark 2.2.13 Let $U<\mathrm{GL}_{m}(\mathbb{Q})$ be finite such that $C:=\operatorname{End}(\bar{U})$ is a field. Denote by $K$ the maximal totally real subfield of $C$. Further suppose that $L_{1}, \ldots, L_{s}$ represent the isomorphism classes of $U$-invariant lattices. Finally fix $F_{i} \in \mathcal{F}_{>0}(U)$ and let $R_{i}=$ $\operatorname{End}_{\mathbb{Z} U}\left(L_{i}\right) \subseteq \mathbb{Z}_{C}$ (in most cases $R_{i}=\mathbb{Z}_{C}$ and there exists at least one $i$ where equality holds). Denote by $R_{i}^{+}:=R_{i} \cap K$. The following algorithm finds (up to conjugacy) all finite supergroups $G$ of $U$ of order dividing a given $\ell \in \mathbb{N}$ :

For $1 \leq i \leq s$ let

$$
\begin{aligned}
N_{i} & :=\left\{x \in N_{\mathrm{GL}_{m}(\mathbb{Q})}(U) \mid L_{i} x=L_{i} \text { and } x F_{i} x^{\text {tr }} F_{i}^{-1} \in R_{i}^{*}\right\} \text { and } \\
P_{i} & :=\left\{a R_{i}^{+} \mid a \in K_{>0},\left(L_{i}, a F_{i}\right) \text { is normalized and } \Pi\left(\operatorname{det}\left(L_{i}, a F_{i}\right)\right) \subseteq \tilde{\Pi}(K, \ell)\right\} .
\end{aligned}
$$

Then the group $N_{i}$ acts on $C, K$ and $R_{i}$ via conjugation. Moreover, $P_{i}$ consists of full orbits under this action. Let $S_{i}$ be a set of representatives of these orbits.

Finally let $U_{i}$ be a coset of $\left(R_{i}^{+}\right)_{>0}^{*} / \mathrm{Nr}_{C / K}\left(R_{i}^{*}\right)$ and let

$$
\mathcal{S}:=\left\{\left(L_{i}, u a F_{i}\right) \mid u \in U_{i}, a R_{i}^{+} \in S_{i}, a \in K_{>0}, 1 \leq i \leq s\right\} .
$$

Then every finite supergroup of $U$ of order dividing $\ell$ is conjugate in $N_{\mathrm{GL}_{m}(\mathbb{Q})}(U)$ to some group that fixes one of the lattices in the finite set $\mathcal{S}$.

In particular, the r.i.m.f. supergroups of $U$ are elements of $\{\operatorname{Aut}(L, F) \mid(L, F) \in \mathcal{S}\}$ and the s.i.m.f. supergroups of $U$ are elements of $\left\{\operatorname{Aut}_{K_{j}}(L, F) \mid(L, F) \in \mathcal{S}, 1 \leq j \leq r\right\}$ where $K_{1}, \ldots, K_{r}$ denote the minimal totally complex subfields of $C$.

Proof: Let $G<\mathrm{GL}_{m}(\mathbb{Q})$ be a finite supergroup of $U$ with $|G|$ dividing $\ell$. By Corollary 2.2.12, the group $G$ fixes some $\left(L^{\prime}, F^{\prime}\right) \in \mathcal{Z}(U) \times \mathcal{F}_{>0}(U)$ such that $F^{\prime}$ is integral on $L^{\prime}$ and $\Pi\left(\operatorname{det}\left(L^{\prime}, F^{\prime}\right)\right) \subseteq \tilde{\Pi}(K, \ell)$. Applying the process described in Definition 2.2.4 yields a normalized lattice $\left(\tilde{L}^{\prime}, \tilde{F}^{\prime}\right)$ with $\Pi\left(\operatorname{det}\left(\tilde{L}^{\prime}, \tilde{F}^{\prime}\right)\right) \subseteq \tilde{\Pi}(K, \ell)$.

Now $\tilde{L}^{\prime}=L_{i} c$ for some $1 \leq i \leq s$ and $c \in C$. After replacing $G$ by $G^{c^{-1}}, G$ fixes $\left(L_{i}, F\right)$ where $F:=c \tilde{F}^{\prime} c^{\text {tr }}$. So there exists some $a R_{i}^{+} \in P_{i}$ such that $F=a F_{i}$ (and thus $\left.a \in K_{>0}\right)$. By definition, there exists some $x \in N_{i}$ such that $a^{x} R_{i}^{+} \in S_{i}$. After replacing $G$ by $G^{x^{-1}}$, it fixes $L_{i} x^{-1}=L_{i}$ and $x a F_{i} x^{\text {tr }}=a^{x^{-1}}\left(x F_{i} x^{\text {tr }} F_{i}^{-1}\right) F_{i}=\tilde{a} F_{i}$ for some $\tilde{a} \in K_{>0}$ with $a R_{i}^{+}=\tilde{a} R_{i}^{+}$. Now $a$ or $\tilde{a}$ are defined by the ideal $a R_{i}^{+}$only up to some element of $\left(R_{i}^{+}\right)_{>0}^{*}$. For $y \in R_{i}^{*}$ it follows from Lemma 2.1.9 that $G^{y^{-1}}$ fixes $\left(L_{i} y^{-1}, y \tilde{a} F_{i} y^{\mathrm{tr}}\right)=\left(L_{i}, \operatorname{Nr}_{C / K}(y) \tilde{a} F_{i}\right)$.

So we have shown that $G$ is conjugate (in $N_{\mathrm{GL}_{m}(\mathbb{Q})}(U)$ ) to some group that fixes a lattice in the set $\mathcal{S}$.

The result now follows, if we can show that $\mathcal{S}$ is finite. The set $U_{i}$ is finite by Dirichlet's unit theorem (note that if $K \neq C$ then $\left(R_{i}^{+}\right)^{2} \leq \operatorname{Nr}_{C / K}\left(R_{i}^{*}\right)$ ). The number of isomorphism classes of $\mathbb{Z} U$-invariant lattices is finite by the Jordan-Zassenhaus theorem.

So it remains to prove that $P_{i}$ is finite: Fix $b R_{i}^{+} \in P_{i}$ and let $J_{i}:=\operatorname{Ann}_{R_{i}^{+}}\left(L_{i}^{\#, b F_{i}} / L_{i}\right)$. If $x \in K_{>0}$ such that $x b F_{i}$ is integral on $L_{i}$, then $L_{i} x J_{i} \subseteq L_{i}^{\#, b F_{i}} J_{i} \subseteq L_{i}$. Thus $x \in J^{-1}$. 
To simplify the definition of the $N_{i}$ in the previous remark, one can use the following

Remark 2.2.14 Assume the situation of the previous remark.

(a) If $F_{i}$ is integral on $L_{i}$ and $\operatorname{det}\left(L_{i}, F_{i}\right)=1$ then

$$
\left\{F \in \mathcal{F}_{>0}(U) \mid\left(L_{i}, F\right) \text { is integral }\right\}=\left\{c F_{i} \mid c \in R_{i} \cap K_{>0}\right\} .
$$

(b) If $\left\{F \in \mathcal{F}_{>0}(U) \mid\left(L_{i}, F\right)\right.$ is integral $\}=\left\{c F_{i} \mid c \in R_{i} \cap K_{>0}\right\}$ then

$$
N_{i}=N_{\mathrm{GL}_{m}(\mathbb{Q})}(U) \cap \mathrm{GL}(L)=\left\{x \in N_{\mathrm{GL}_{m}(\mathbb{Q})}(U) \mid L_{i} x=L_{i}\right\} .
$$

Proof:

(a) Suppose $c \in K_{>0}$ such that $c F_{i}$ is integral on $L_{i}$. Then $x c F_{i} y^{\operatorname{tr}} \in \mathbb{Z}$ for all $x, y \in L_{i}$. Hence $L_{i} c \subseteq L_{i}^{\#, F_{i}}=L_{i}$ and therefore $c \in R_{i}$.

(b) Let $x \in N_{\mathrm{GL}_{m}(\mathbb{Q})}(U) \cap \mathrm{GL}(L)$. Then $x F_{i} x^{\text {tr }} \in \mathcal{F}_{>0}(U)$. Hence there exists some $c \in K_{>0}$ such that $x F_{i} x^{\mathrm{tr}}=c F_{i}$. Now $x F x^{\mathrm{tr}}$ is integral on $L_{i} x^{-1}=L_{i}$. This shows $c \in R_{i}^{+}$. From $\operatorname{det}(c)=1$ it follows that $\mathrm{Nr}_{K / \mathbb{Q}}(c)=1$ and thus $c \in\left(R_{i}^{+}\right)^{*}$. This proves $x \in N_{i}$.

Note that there does not always exist some $F_{i}$ such that the condition of (b) holds.

\subsubsection{Quaternion algebras as endomorphism rings}

We now turn to the case where a s.i.m.f. matrix group $G$ contains an irreducible normal subgroup $N$ such that $\operatorname{End}(\bar{N})$ is a quaternion algebra $\mathcal{Q}$ with center $K$.

If $N=F^{*}(G)$, then $\mathcal{Q}$ will frequently be a totally definite quaternion algebra (i.e. $\mathcal{Q}$ is ramified at all infinite places of $K)$. In this case the structure of $G$ is rather limited.

Theorem 2.2.15 Let $G<\operatorname{Sp}_{2 n}(\mathbb{Q})$ be s.p.i.m.f.. Suppose that $E:=\operatorname{End}\left(\overline{F^{*}(G)}\right)$ is a totally definite quaternion algebra with center $K$. Then $G$ acts on $E$ and $K$ by conjugation. Let

$$
\begin{aligned}
& S:=\{g \in G \mid g x=x g \text { for all } x \text { in } K\} \text { and } \\
& B:=\mathcal{B}^{o}\left(F^{*}(G)\right)=\{g \in G \mid g x=x g \text { for all } x \text { in } E\}
\end{aligned}
$$

be the kernels of these actions. Then

$$
1 \unlhd F^{*}(G) \unlhd B \unlhd S \unlhd G .
$$

Further $G / S$ is isomorphic to a subgroup of $\operatorname{Gal}(K / \mathbb{Q})$ and $S / B$ has exponent 1 or 2 (and is abelian). 
Proof: Only the claim that $S / B$ has exponent 1 or 2 is not obvious. The proof given in [MGH98, Theorem 4] applies mutatis mutandis.

With the notation from above, the next lemma can be used to extend $B$ by some element from $S$.

Lemma 2.2.16 Let $N=\mathcal{B}^{\circ}(N)<\mathrm{GL}_{m}(\mathbb{Q})$ be finite such that $Q:=\operatorname{End}(\bar{N})$ is a quaternion algebra with center $K$. Suppose $x, y \in \mathrm{GL}_{m}(\mathbb{Q})$ induce the same outer automorphism on $N$ and $x^{2}, y^{2} \in N$. If $x$ commutes with $K$ then

(a) $\operatorname{End}(\overline{\langle N, x\rangle})=C_{Q}(x) \simeq K[X] /\left(X^{2}-d\right)$ for some $d \in K$.

(b) There exist some $g \in N$ and $c \in Q$ such that $y=g c x$. In particular, $y$ commutes with $K$. Then $\operatorname{End}(\overline{\langle N, y\rangle}) \simeq K[X] /\left(X^{2}-\operatorname{nr}_{Q / K}(c) d\right)$ where $\operatorname{nr}_{Q / K}$ denotes the reduced norm on $Q$. Further $\operatorname{nr}_{Q / K}(c)$ is a root of unity in $\mathbb{Z}_{K}$.

(c) If $C_{Q}(x)$ is a field and $\operatorname{nr}_{Q / K}(c) \in\left(\mathbb{Z}_{K}^{*}\right)^{2}$ then $\langle N, x\rangle$ and $\langle N, y\rangle$ are conjugate.

Proof:

(a) For any $z \in Q$ we have $z^{x} \in \operatorname{End}\left(\overline{N^{x}}\right)=\operatorname{End}(\bar{N})=Q$. Hence $x$ induces a $K$ automorphism on $Q$. By the Skolem-Noether theorem there exists some $a \in Q^{*}$ such that $z^{a}=z^{x}$ for all $z \in Q$. It follows from $x \notin C_{\mathrm{GL}_{n} \mathbb{Q}}(Q)$ that

$$
K \subsetneq K[a] \subseteq C_{Q}(a)=C_{Q}(x) \subsetneq Q
$$

Thus $K[a]=C_{Q}(x)$. Since $x^{2} \in N$, it induces the identity on $Q$. Hence $a^{2} \in K$ and therefore $K[a] \cong K[X] /\left(X^{2}-d\right)$ where $d:=-\operatorname{nr}_{Q / K}(a) \in K$.

(b) Since $y x^{-1}$ induces an inner automorphism on $N$, it is contained in $N Q^{*}$. It follows from part (a) that

$$
\operatorname{End}(\overline{\langle N, y\rangle})=\operatorname{End}(\overline{\langle N, c x\rangle}) \simeq K[X] /\left(X^{2}+\operatorname{nr}_{Q / K}(c a)\right)
$$

as claimed.

Further $c x c x^{-1} \in\left(g^{-1} y\right)^{2} x^{-2} \in N$ has finite order. Hence the reduced norm $\operatorname{nr}_{Q / K}(c)^{2}=\operatorname{nr}_{Q / K}\left(c \cdot\left(x c x^{-1}\right)\right) \in K^{*}$ also has finite order.

(c) Let $u \in \mathbb{Z}_{K}^{*}$ such that $\operatorname{nr}_{Q / K}(c)=u^{2}$. Thus $u \in \mathbb{Z}_{K}^{*}$ has finite order which implies $u \in \mathcal{B}^{\circ}(N)=N$. By the above, both $a$ and $u^{-1} c a$ have vanishing traces. So $\mathrm{nr}_{Q / K}\left(u^{-1} c\right)=1$ implies that $a$ and $u^{-1} c a$ have the same minimal polynomial (over $K$ ). It follows from the Skolem-Noether theorem that there exists some $t \in Q^{*}$ such that $a^{t}=u^{-1} c a$. Finally

$$
x^{t}=t^{-1} x t x^{-1} x=t^{-1} a t a^{-1} x=u^{-1} c_{a a^{-1}} x=(g u)^{-1} y
$$

shows $\langle N, x\rangle^{t}=\left\langle N,(g u)^{-1} y\right\rangle=\langle N, y\rangle$. 
In most cases, we will apply this result to the following situation.

Remark 2.2.17 Let $N<\operatorname{Sp}_{2 n}(\mathbb{Q})$ be finite such that $Q:=\operatorname{End}(\bar{N})$ is a quaternion skewfield with center $K$. If $\mathcal{B}^{o}(N)$ is not s.p.i.m.f., then the above lemma allows us to construct (up to conjugacy) all s.p.i.m.f. supergroups $G \triangleright N$ satisfying one of the following conditions:

- $C_{G}(N) \nsubseteq \mathcal{B}^{o}(N)$

- $\mathcal{B}^{o}(N)$ has even index in $\{g \in G \mid g x=x g$ for all $x \in K\}$.

(Note that this condition holds for example if $N=F^{*}(G)$ is assumed and $Q$ is a totally definite quaternion algebra over $\mathbb{Q}$ as Theorem 2.2 .15 shows).

More precisely, one can use the following algorithm:

(a) Set $X:=\emptyset$.

(b) If $N=F^{*}(G)$ is assumed skip this step. Otherwise let $\mathfrak{M}_{1}, \ldots, \mathfrak{M}_{r}$ be representatives of the conjugacy classes of maximal $\mathbb{Z}_{K}$-orders in $Q$. Further let $\mathfrak{M}_{i}^{* .1}=\left\{x \in \mathfrak{M}_{i} \mid \operatorname{nr}_{Q / K}(x)=1\right\}$ be the torsion subgroup of $\mathfrak{M}_{i}^{*}$. (See [KV] for algorithms to compute these objects).

Include to the set $X$ all elements $x \in \mathfrak{M}_{i}^{*, 1}$ whose order is a prime power greater than 2, provided that $X$ does not already have an element of the same order.

(c) Let $U$ be the torsion subgroup of $\mathbb{Z}_{K}^{*} \cap \operatorname{nr}_{Q / K}\left(Q^{*}\right)$. For any $u U^{2}$ in $U / U^{2}$ find some $c_{u}$ with $u=\operatorname{nr}_{Q / K}(c)$. Let $C$ be the set of the $c_{u}$. (In most cases, $Q$ will be a totally definite quaternion algebra, so one can choose $C=\{1\}$ ).

(d) For each class of outer automorphisms in $\operatorname{Out}(N)$ that is not realized in $\mathcal{B}^{\circ}(N)$ but its square is, compute one $x \in \mathrm{GL}_{2 n}(\mathbb{Q})$ that realizes this automorphism.

If such an $x$ exists, then include $\{c x \mid c \in C\}$ to $X$.

(e) Each group $G$ satisfying the hypothesis contains (up to conjugacy) a subgroup $\langle N, x\rangle$ for some $x \in X$.

Since $\operatorname{End}\left(\overline{\left\langle\mathcal{B}^{\circ}(N), x\right\rangle}\right)$ is a field, one can use the m-parameter argument (Corollary 2.2.12 to construct a representative of the conjugacy class of $G$.

Proof: We have to show that each $G$ satisfying the hypothesis contains a subgroup conjugate to $\left\langle\mathcal{B}^{o}(N), x\right\rangle$ for some $x \in X$.

Suppose first that there exists some $g \in C_{G}(N)=C_{G}\left(\mathcal{B}^{o}(N)\right)$ such that $g \notin \mathcal{B}^{o}(N)$. Taking appropriate powers, we can assume that the order of $g$ is a prime power different from 2 since $-I_{2 n} \in \mathcal{B}^{o}(N)$. So $g$ is conjugate (in $Q$ ) to some $x \in X$. Hence $\left\langle\mathcal{B}^{o}(N), g\right\rangle$ and $\left\langle\mathcal{B}^{\circ}(N), x\right\rangle$ are conjugate in $Q$.

Suppose now $C_{G}(N)=C_{G}\left(\mathcal{B}^{o}(N)\right) \subseteq \mathcal{B}^{o}(N)$. Then by the assumption, there exists some $g \in G \backslash \mathcal{B}^{o}(N)$ that commutes with $K$ and $g^{2} \in \mathcal{B}^{o}(N)$. So the claim follows from Lemma 2.2.16. 


\subsection{Algorithms}

Let $G<\mathrm{GL}_{m}(\mathbb{Q})$ be finite. After a change of bases, we may suppose that $G<\mathrm{GL}_{m}(\mathbb{Z})$. We first explain how to compute representatives for the isomorphism classes of $G$ invariant lattices. Clearly $\mathcal{Z}(G)$ is closed under taking sums and intersections.

Suppose $L^{\prime} \subseteq L \in \mathcal{Z}(G)$. Let $M_{1}, \ldots, M_{s}$ be the nontrivial $p$-Sylow groups of $L / L^{\prime}$. Then $G$ acts on $L / L^{\prime}$ as automorphisms of groups. In particular $L^{\prime}=\bigcap_{i=1}^{s} L_{i}$ is the intersection of the $G$-invariant lattices $L_{i}=\oplus_{j \neq i}\left(L^{\prime}+M_{j}\right)$ whose index in $L$ is a prime power. Moreover, this intersection is unique.

Algorithm 2.3.1 ([PH84]) Let $\Lambda \subseteq \mathbb{Z}^{m \times m}$ be a $\mathbb{Z}$-order in a simple subalgebra $A$ of $\mathbb{Q}^{m \times m}$. Further let $L=\mathbb{Z}^{1 \times m}$ be the natural $\Lambda$-lattice. This algorithm returns all $\Lambda$-invariant sublattices of $L$ that contain $L p^{k}$ for some $k \geq 1$ and a fixed prime $p$.

Input: Generators of the natural representation of $\Lambda$ and a prime $p$.

(a) Using the meataxe (see [Par84]), find all $p$-modular constituents of the natural representation of $\Lambda$ and the corresponding simple $\Lambda / p \Lambda$-modules $S_{1}, \ldots, S_{k}$.

(b) For each $\Lambda$ - invariant lattice $M$ found so far, compute all maximal $\Lambda$ - invariant sublattices of $M$ as kernels of $\Lambda / p \Lambda$-epimorphisms $M \rightarrow S_{i}$ for some $1 \leq i \leq s$.

(c) One continues the algorithm with these newly constructed lattices, provided they are not a scalar multiple of a lattice computed before.

Remark 2.3.2 ([Neb95, III.11]) In general, the above algorithm does not terminate. One has to specify some additional stopping conditions as follows.

Let $\Lambda:=\langle G\rangle_{\mathbb{Z}}$ for some finite rationally irreducible subgroup $G<\mathrm{GL}_{m}(\mathbb{Z})$ where $E:=\operatorname{End}(\bar{G})$ is a field. Since $\Lambda^{\prime}:=\left\langle\Lambda, \mathbb{Z}_{E}\right\rangle_{\mathbb{Z}}$ is an order, there exists some $L \in \mathcal{Z}(G)$ such that $\operatorname{End}_{\Lambda}(L)=\mathbb{Z}_{K}$. So without loss of generality $L=\mathbb{Z}^{1 \times m}$.

Denote by $p_{1}, \ldots, p_{s}$ the prime divisors of $|G|$ and let $\mathfrak{p}_{i, 1}, \ldots, \mathfrak{p}_{i, n_{i}}$ be the prime ideals over $p_{i} \mathbb{Z}_{K}$. These prime ideals act on the $\Lambda^{\prime}$-invariant sublattices of $L$ whose index is a power of $p_{i}$. Using the above algorithm, we find representatives $L_{i, 1}, \ldots, L_{i, m_{i}}$ of the corresponding orbits.

Let $\mathfrak{a}_{1}, \ldots, \mathfrak{a}_{h}$ be representatives of $\mathcal{C l}\left(\mathbb{Z}_{K}\right)$. Then

$$
\mathcal{S}^{\prime}:=\left\{\left(\bigcap_{i=1}^{s} L_{i, j_{i}}\right) \mathfrak{a}_{j} \mid 1 \leq j \leq h, 1 \leq i_{j} \leq m_{i}\right\}
$$

is finite and it contains a system of representatives for the isomorphism classes of $\Lambda^{\prime}$-invariant lattices.

Using the above algorithm again, for each $L^{\prime} \in \mathcal{S}^{\prime}$ one computes the finite set

$$
\mathcal{Z}_{o}\left(L^{\prime}\right):=\left\{M=\bigcap_{i=1}^{s} M_{i} \mid M_{i} \in \mathcal{Z}(G), M_{i} \leq L^{\prime}, L^{\prime} / M_{i} \text { a } p_{i} \text {-group and } M \mathbb{Z}_{E}=L^{\prime}\right\} .
$$

Then $\mathcal{S}:=\bigcup_{L^{\prime} \in \mathcal{S}^{\prime}} \mathcal{Z}_{o}\left(L^{\prime}\right)$ contains a representative of each isomorphism class of $\mathcal{Z}(G)$. 
Proof: By the Jordan-Zassenhaus theorem ([Rei03, Chapter 26]) the number of isomorphism classes of $\Lambda^{\prime}$-invariant lattices is finite. So $\mathcal{S}^{\prime}$ is finite. Suppose $L^{\prime} \leq L$ is $\Lambda^{\prime}$-invariant, then [Rei03, Theorems 41.1 and 41.7] show that each prime $p$ not dividing $|G|$, does not split $\bar{G}$ and the completion $\Lambda_{p}^{\prime}:=\Lambda^{\prime} \otimes_{\mathbb{Z}} \mathbb{Z}_{p}$ is a maximal order. Hence the completions $L^{\prime} \otimes_{\mathbb{Z}} \mathbb{Z}_{p}$ and $L \otimes_{\mathbb{Z}} \mathbb{Z}_{p}$ are locally isomorphic. So there exists some fractional ideal $\mathfrak{a}$ of $\mathbb{Z}_{E}$ such that $L^{\prime} \mathfrak{a} \leq L$ and $\left|L / L^{\prime} \mathfrak{a}\right|$ is a product of the $p_{i}$. Then it follows from the definition of $\mathcal{S}^{\prime}$ that $L^{\prime} \mathfrak{a}$ is isomorphic to some lattice in $\mathcal{S}^{\prime}$.

Suppose now $L^{\prime} \in \mathcal{S}^{\prime}$ and $M \in \mathcal{Z}_{o}\left(L^{\prime}\right)$. Then there are only finitely many isomorphism classes of such lattices $M$. Further $x \in E$ satisfies $M x \in \mathcal{Z}_{o}\left(L^{\prime}\right)$ if and only if $x \in \mathbb{Z}_{E}^{*}$. Since $\left[\mathbb{Z}_{E}^{*}: \operatorname{End}_{\Lambda}(M)^{*}\right]$ is finite, it follows that $\mathcal{Z}_{o}\left(L^{\prime}\right)$ is finite.

Suppose now $L^{\prime} \in \mathcal{Z}(G)$. Then there exists some $x \in E$ such that $L^{\prime} \mathbb{Z}_{E} x \in \mathcal{S}^{\prime}$. Furthermore $\left|L^{\prime} \mathbb{Z}_{E} x / L^{\prime} x\right|$ is a product of the $p_{i}$ since $\operatorname{End}_{\Lambda_{p}}\left(L_{p}^{\prime}\right)$ is maximal for all primes $p$ not dividing $|G|$. Hence $L^{\prime} x \in \mathcal{Z}_{o}\left(L^{\prime} \mathbb{Z}_{E} x\right) \subseteq \mathcal{S}$ as claimed.

In [PS97, Plesken and Souvignier describe an algorithm which computes automorphism groups of lattices. Together with the Minkowski bound (see Lemma 2.2.9), the m-parameter argument (see Remark 2.2.13) and the above algorithm we can thus find (up to conjugacy) all r.i.m.f. or s.i.m.f. supergroups of any finite matrix group $G$ where $\operatorname{End}(\bar{G})$ is a field. Provided that we can test whether two given groups are conjugate:

Algorithm 2.3.3 The following algorithm tests whether two given s.i.m.f. groups $G_{1}, G_{2}<\operatorname{Sp}_{2 n}(\mathbb{Q})$ are conjugate. If so, it returns some $x \in \mathrm{GL}_{2 n}(\mathbb{Q})$ such that $G_{2}=G_{1}^{x}$. For $i=1,2$ let $E_{i}:=\operatorname{End}\left(\overline{G_{i}}\right)$.

- Let $d:=\min \left\{\operatorname{det}(L, F) \mid F \in \mathcal{F}_{>0}\left(G_{1}\right)\right.$ is primitive on $\left.L \in \mathcal{Z}\left(G_{1}\right)\right\}$ and fix some primitive pair $(L, F) \in \mathcal{Z}\left(G_{1}\right) \times \mathcal{F}_{>0}\left(G_{1}\right)$ such that $\operatorname{det}(L, F)=d$.

- Let $L_{1}^{\prime}, \ldots, L_{s}^{\prime}$ be representatives the isomorphism classes of $\mathcal{Z}\left(G_{2}\right)$.

- Two elements in

$$
\mathcal{L}^{\prime}:=\bigcup_{1=1}^{s}\left\{\left(L_{i}^{\prime}, F^{\prime}\right) \mid F^{\prime} \in \mathcal{F}_{>0}\left(G_{2}\right) \text { is primitive on } L_{i}^{\prime} \text { and } \operatorname{det}\left(L_{i}^{\prime}, F^{\prime}\right)=d\right\}
$$

are said to be equivalent if there exists some isometry $t$ between them such that $E_{2}^{t}=E_{2}$. Let $S$ be a set of representatives of the equivalence classes.

- If there exists some isometry $x$ between $(L, F)$ and some element of $S$ such that $E_{1}^{x}=E_{2}$ then return $x$ otherwise return false.

An algorithm for the required isometry tests is also given in [PS97].

Proof: It follows from the proof of Remark 2.2.13 that $\mathcal{L}^{\prime}$ is finite. Thus the algorithm terminates. Suppose first that there exists some $x \in G_{2 n}(\mathbb{Q})$ such that $\left(L x, x^{-1} F x^{-\mathrm{tr}}\right) \in S$ and $E_{1}^{x}=E_{2}$. Then by maximality

$$
G_{1}^{x}=\left(\operatorname{Aut}_{E_{1}}(L, F)\right)^{x}=\operatorname{Aut}_{E_{1}^{x}}\left(L x, x^{-1} F x^{-\operatorname{tr}}\right)=G_{2} .
$$


Conversely suppose that $G_{2}=G_{1}^{y}$ for some $y \in \mathrm{GL}_{2 n}(\mathbb{Q})$. Then $\left(L y, y^{-1} F y^{-\mathrm{tr}}\right) \in$ $\mathcal{Z}\left(G_{2}\right) \times \mathcal{F}_{>0}\left(G_{2}\right)$ is primitive and has determinant $d$. So there exists some $e \in E_{2}$ such that $\left(\right.$ Lye, $\left.(y e)^{-1} F(y e)^{- \text {tr }}\right) \in \mathcal{L}^{\prime}$. By definition of $S$, there exists some $t \in \mathrm{GL}_{2 n}(\mathbb{Q})$ such that $E_{2}^{t}=E_{2}$ and (Lyet, $\left.(\text { yet })^{-1} F(\text { yet })^{-\operatorname{tr}}\right) \in S$. So $x:=$ yet furnishes an isometry between $(L, F)$ and some element of $S$ satisfying $E_{1}^{x}=E_{2}$ as claimed.

\subsection{Some notation}

Definition and Remark 2.4.1 For $i=1,2$ let $G_{i}<\mathrm{GL}_{m_{i}}(\mathbb{Q})$ be finite irreducible matrix groups and let $E_{i}:=\operatorname{End}\left(\overline{G_{i}}\right)$. The tensor product

$$
G_{1} \otimes G_{2}:=\left\{g_{1} \otimes g_{2} \mid g_{i} \in G_{i}\right\}<\mathrm{GL}_{m_{1} m_{2}}(\mathbb{Q})
$$

is isomorphic to some central product $G_{1} Y_{C} G_{2}$. This matrix group is usually not irreducible, since $E_{1} \otimes E_{2}$ might not be a skewfield. The following definition is used to construct a (usually irreducible) direct summand of $G_{1} \otimes G_{2}$.

Let $Q$ be a maximal common subalgebra of $E_{1}$ and $E_{2}$ of dimension $d=\operatorname{dim}_{\mathbb{Q}}(Q)$. Then $G_{1} \underset{Q}{\otimes} G_{2}:=\triangle^{\mathbb{Q}}\left(\triangle_{Q}\left(G_{1}\right) \otimes \triangle_{Q}\left(G_{2}\right)\right)<\operatorname{GL} \frac{m_{1} m_{2}}{d}(\mathbb{Q})$ is isomorphic to $G_{1} Y_{C} G_{2}$ with $C=G_{1} \cap Q \cap G_{2}$. Moreover $G_{1} \underset{Q}{\otimes} G_{2}$ contains a normal subgroup $H_{i}$ isomorphic to $G_{i}$ where the restriction of the natural character onto $H_{i}$ is a multiple of the natural character of $G_{i}$. To simplify the notation, we use the following conventions: If $Q \simeq \mathbb{Q}(\alpha)$ is a field, we write $\underset{\alpha}{\otimes}$ instead of $\underset{\mathbb{Q}(\alpha)}{\otimes}$. If $Q \simeq \mathcal{Q}_{\alpha, P_{1}, \ldots, P_{r}}$ is a quaternion algebra with center $K$ ramified only at the (finite or infinite) places $P_{i}$, we write $\underset{P_{1}, \ldots, P_{r}}{\otimes}$ if $K=\mathbb{Q}$ and we write $\underset{\alpha, P_{1}, \ldots, P_{r}}{\otimes}$ if $K \simeq \mathbb{Q}(\alpha)$. Finally, if $d=m_{2}$, then $G_{2}$ embeds into $E_{1}^{*}$. In this case, we use $\circ$ instead of $\underset{Q}{\otimes}$.

Note that this construction does not always give irreducible matrix groups. We will only encounter the following examples (in dimension 16) where this is not the case: In these cases (after exchanging the $G_{i}$ ) we have $G_{1} \simeq C_{10}, m_{1}=4$ and $E_{1} \simeq \mathbb{Q}\left(\zeta_{10}\right)$. Further $E_{2}$ is a totally definite quaternion algebra which is split by $E_{1}$. Thus the maximal common subalgebra of $E_{1}$ and $E_{2}$ equals $\mathbb{Q}$ and $G_{1} \otimes G_{2}$ is reducible. In these cases, we make a slight abuse of notation and denote by $G_{1} \otimes G_{2}$ an irreducible direct summand of $G_{1} \otimes G_{2}$ (this was first introduced in [NP95, page 91]). This notation has the advantage, that in all our examples the dimension formula still holds, that is: $G_{1} \underset{\sqrt{5}^{\prime}}{\otimes} G_{2}$ denotes a subgroup of $\mathrm{GL}_{k}(\mathbb{Q})$ where $k=2 m_{2}=\frac{m_{1} m_{2}}{\operatorname{dim}_{\mathbb{Q}}(\mathbb{Q}(\sqrt{5}))}$. 
Definition and Remark 2.4.2 We will use the following conventions for the names (of the conjugacy classes) of rational/symplectic/quaternionic matrix groups.

- If $G<\mathrm{GL}_{m}(Q)$ for some skewfield $Q$, then $\pm G:=\left\langle G,-I_{m}\right\rangle<\mathrm{GL}_{m}(Q)$.

- The symbols $A_{n}, B_{n}, F_{4}=D_{4}, E_{6}, E_{7}, E_{8}$ denote (the automorphism groups of) the corresponding root lattices.

- If a maximal finite irreducible rational/symplectic/quaternionic primitive matrix group $G<\mathrm{GL}_{m}(\mathbb{Q})$ is not a tensor product of rational, symplectic or quaternionic matrix groups of smaller dimension, it is denoted by ${ }_{E}[\mathrm{Con}]_{k}$ where $E \simeq \operatorname{End}(\bar{G})$ and $m=k \cdot \operatorname{dim}_{\mathbb{Q}}(E)$ since $G$ can be identified with a subgroup of $\mathrm{GL}_{k}(E)$. Further, Con describes some construction of $G$ from building blocks $\left(O_{p}\right.$ and components; see Tables 2.5.2 and 2.5.1) or smaller matrix groups by taking generalized Bravais groups, tensor products or group extensions.

Again, if $E \simeq \mathbb{Q}$ we omit the subscript $E$. If $E \simeq \mathbb{Q}(\sqrt{\alpha})$ is a field, we write ${ }_{\alpha}[\mathrm{Con}]_{k}$ and if $E \simeq \mathcal{Q}_{\alpha, P_{1}, \ldots, P_{r}}$ is a quaternion algebra over $K$ ramified only at the places $P_{i}$ we write $P_{1}, \ldots, P_{r}[C o n]_{k}$ if $K=\mathbb{Q}$ and ${ }_{\alpha, P_{1}, \ldots, P_{r}}[C o n]_{k}$ if $K \simeq \mathbb{Q}(\alpha)$.

- If $G$ is symplectic imprimitive, then it is conjugate to the wreath product of some s.p.i.m.f. subgroup $H<\operatorname{Sp}_{\frac{2 n}{k}}(\mathbb{Q})$ with $S_{k}$. In this case, we write $H^{k}$.

These conventions are consistent with the ones of [Ple91, NP95, Neb95, Neb96, Neb98]. Moreover, for matrix groups described in loc. cit. we use the names given there.

\section{Example 2.4.3}

- $C_{30}$ denotes the torsion subgroup of $\mathbb{Q}\left(\zeta_{30}\right)^{*}$ which gives rise to a subgroup of $\mathrm{GL}_{8}(\mathbb{Q})$ with $\mathbb{Q}\left(\zeta_{30}\right)$ as commuting algebra. Clearly, there exists only one split extension of this matrix group by $C_{4}$ that fixes $\mathbb{Q}(\sqrt{-15})$. One finds that this group is s.p.i.m.f. and we denote it by $\sqrt{-15}\left[C_{30}: C_{4}\right]_{4}<\operatorname{Sp}_{8}(\mathbb{Q})$.

- The group $D_{8} \otimes C_{4}$ is a subgroup of $\mathrm{GL}_{4}(\mathbb{Q})$ and it is one of the building blocks described in Table 2.5.2. Taking its generalized Bravais group, one obtains a s.p.i.m.f. subgroup of $\mathrm{Sp}_{4}(\mathbb{Q})$ denoted by its isomorphism type ${ }_{i}\left[\left(D_{8} \otimes C_{4}\right) \cdot S_{3}\right]_{2}$. Tensoring this group with $\operatorname{Aut}\left(A_{2}\right)<\mathrm{GL}_{2}(\mathbb{Q})$ gives a s.p.i.m.f. subgroup of $\mathrm{Sp}_{8}(\mathbb{Q})$ which we call ${ }_{i}\left[\left(D_{8} \otimes C_{4}\right) \cdot S_{3}\right]_{2} \otimes A_{2}$.

Lemma 2.1.21 shows that taking wreath products of these two s.p.i.m.f. groups also yields maximal finite groups denoted by ${ }_{i}\left[\left(D_{8} \otimes C_{4}\right) \cdot S_{3}\right]_{2}^{k}<\operatorname{Sp}_{4 k}(\mathbb{Q})$ and $\left({ }_{i}\left[\left(D_{8} \otimes C_{4}\right) \cdot S_{3}\right]_{2} \otimes A_{2}\right)^{\ell}<\operatorname{Sp}_{8 \ell}(\mathbb{Q})$.

As in the classification of the maximal finite rational and quaternionic matrix groups, we frequently have to construct a matrix group $H$ that contains an irreducible subgroup $G_{1} \otimes G_{Q}$ of index 2. Most of these extensions come from one of the following three constructions: 
Definition and Remark 2.4.4 ([NP95, (II.4) Proposition]) For $i=1,2$ let $G_{i}<\mathrm{GL}_{m_{i}}(\mathbb{Q})$ be two finite irreducible matrix groups and let $E_{i}:=\operatorname{End}\left(\overline{G_{i}}\right)$. Further let $Q$ be a maximal common subalgebra of $E_{1}$ and $E_{2}$ such that $G_{1} \underset{Q}{\otimes} G_{2}<\mathrm{GL}_{m}(\mathbb{Q})$ is irreducible. We view $G_{i}$ as a subgroup of $G_{1} \underset{Q}{\otimes G_{2}}$ and $\overline{G_{i}}$ as a subalgebra of $\overline{G_{1} \otimes G_{2}}$.

(a) Suppose there exist some units $a_{i} \in \overline{G_{i}} \backslash G_{i}$ and a positive squarefree integer $p$ such that $G_{i}^{a_{1}}=G_{i}$ and $p^{-1} a_{i}^{2} \in G_{i}$. Then

$$
G_{1}^{2(p)} \underset{Q}{\otimes} G_{2}:=\left\langle\underset{Q}{\left.G_{1} \underset{Q}{\otimes} G_{2}, p^{-1} a_{1} a_{2}\right\rangle}\right.
$$

is an irreducible subgroup of $\mathrm{GL}_{m}(\mathbb{Q})$ that contains $G_{1} \underset{Q}{\otimes G_{2}}$ with index 2 .

(b) Suppose $G_{1} \underset{Q}{\otimes} G_{2} \subseteq A \subset B \subseteq \mathbb{Q}^{m \times m}$ is a chain of simple algebras with $B=A \oplus x A$ for some $x \in B$ such that $x^{2}= \pm 1, x A=A x$ and $x \overline{G_{i}}=\overline{G_{i}} x$. If there exist some units $a_{i} \in \overline{G_{i}}$ and a positive squarefree integer $p$ such that $G_{i}^{a_{i} x}=G_{i}$ and $p^{-1}\left(a_{i} x\right)^{2} \in G_{i}$ then

$$
G_{1} \underset{Q}{\stackrel{2}{\otimes}} G_{2}:=\left\langle\underset{Q}{G_{1}} \underset{Q}{\otimes} G_{2}, p^{-1} a_{1} a_{2} x\right\rangle
$$

is an irreducible subgroup of $\mathrm{GL}_{m}(\mathbb{Q})$ that contains $\underset{Q}{G_{1}} \underset{Q}{\otimes} G_{2}$ with index 2 .

(c) Suppose $A \subseteq C_{\mathbb{Q}^{m \times m}}\left(\overline{G_{2}}\right)$ is a simple subalgebra that contains $\overline{G_{1}}$. If there exist units $a_{1} \in \bar{A}, a_{2} \in \overline{G_{2}}$ and a positive squarefree integer $p$ such that $G_{i}^{a_{i}}=G_{i}$ and $p^{-1} a_{i}^{2} \in G_{i}$, then

$$
G_{1} \underset{Q}{\stackrel{2(p)}{\otimes}} G_{2}:=\left\langle G_{1} \underset{Q}{\otimes} G_{2}, p^{-1} a_{1} a_{2}\right\rangle
$$

is an irreducible subgroup of $\mathrm{GL}_{m}(\mathbb{Q})$ that contains $\underset{\mathbb{Q}}{G_{1}} \underset{Q}{\otimes} G_{2}$ with index 2 .

In addition, the symbols are simplified as follows:

- If $m_{2}=\operatorname{dim}_{\mathbb{Q}}(Q)$, we write $\stackrel{2(p)}{\circ}, \stackrel{2(p)}{\square}$ and $\stackrel{2(p)}{\square}$ instead of $\underset{Q}{\stackrel{2(p)}{\otimes}} \underset{Q}{\stackrel{2(p)}{\otimes}}$ and $\underset{Q}{\stackrel{2(p)}{\otimes}}$ respectively.

- If $Q \simeq \mathbb{Q}$, the subscript $Q$ is completely omitted. If $Q \simeq \mathbb{Q}(\alpha)$ is a field, we write $\alpha$ instead of $Q$. If $Q \simeq \mathcal{Q}_{\alpha, P_{1}, \ldots, P_{s}}$ is a quaternion algebra with center $K$ ramified only at the places $P_{1}, \ldots, P_{r}$, we replace the subscript $Q$ by $P_{1}, \ldots, P_{s}$ if $K=\mathbb{Q}$ or by $\alpha, P_{1}, \ldots, P_{s}$ if $K \simeq \mathbb{Q}(\alpha)$.

- If $p=1$, we omit $(p)$ in the above symbols.

In most cases, two different extensions of $G_{1} \underset{Q}{\otimes G_{2}}$ by $C_{2}$ can be distinguished by these symbols together with their commuting algebras. Note however, this is not always the case due to the solvability of certain relative norm equations: 
Example 2.4.5 Let $G_{1}={ }_{\sqrt{5}, \infty}\left[\mathrm{SL}_{2}(5)\right]_{1}$ be the torsion subgroup of any maximal order of the quaternion algebra $\mathcal{Q}_{\sqrt{5}, \infty}$ with center $\mathbb{Q}(\sqrt{5})$ ramified only at the two infinite places. Since its commuting algebra is another copy of $\mathcal{Q}_{\sqrt{5}, \infty}$, we find some $x \in E_{1}$ of order 4 . Let $G_{2}$ be the subgroup generated by $x$. Thus $G:=G_{1} \circ G_{2}$ denotes an irreducible subgroup of $\mathrm{GL}_{8}(\mathbb{Q})$ with commuting algebra $E \simeq \mathbb{Q}(i, \sqrt{5})$. Since $\operatorname{Out}\left(G_{1}\right) \simeq \operatorname{Out}\left(G_{2}\right) \simeq C_{2}$ there exists only one class of outer automorphism that acts nontrivially on $G_{1}$ and $G_{2}$. It can be represented by an automorphism of order 2 , thus there exists only two possible such extensions.

Construction (b) can be used to find some $\alpha=a_{1} a_{2} x$ that acts like the nontrivial outer automorphism on the $G_{i}$. Clearly $H_{1}:=\langle G, \alpha\rangle$ has commuting algebra $\mathbb{Q}(\sqrt{-5})$. But then $u:=\frac{1+\sqrt{5}}{2} \in E$ satisfies $\operatorname{Nr}_{E / \mathbb{Q}(\sqrt{-5})}(u)=u \cdot u^{\alpha}=-1$. In particular $H_{2}:=\langle G, u \alpha\rangle$ is an extension of $G$, not isomorphic to $H_{1}$ but with the same commuting algebra.

Remark 2.4.6 If (like in the previous example) there exist only two s.i.m.f. extensions one split and one not, we write $2_{+}(p)$ for the split and $2_{-}(p)$ for the nonsplit extension. Thus the groups $H_{i}$ from above are labeled $\sqrt{5}, \infty\left[\mathrm{SL}_{2}(5)\right]_{1}{ }_{\square+} C_{4}$ and $\sqrt{5, \infty}\left[\mathrm{SL}_{2}(5)\right]_{1}{ }_{\square}-C_{4}$ (see Theorem 4.5.1).

If there are several such extensions yielding s.i.m.f. groups that cannot be distinguished by the above, we describe the automorphisms induced by $p^{-1} a_{1} a_{2}$ or $p^{-1} a_{1} a_{2} x$ explicitly when the groups are introduced for the first time.

Frequently, one has some s.p.i.m.f. matrix group $G$ that contains an irreducible subgroup $N:=G_{1} \otimes_{Q} G_{2}$ of index 2 and one wants to know whether $G$ can be obtained from $N$ by one of the constructions above. We want to discuss one example, to give some idea how this can be done. Clearly (b) is the most difficult construction, since it requires to find 4 parameters $p, a_{1}, a_{2}, x$ whereas the others only require 3 .

Example 2.4.7 Suppose $G<\mathrm{Sp}_{8}(\mathbb{Q})$ is s.p.i.m.f. and it contains a normal subgroup $N:=\sqrt{-2}\left[\mathrm{GL}_{2}(3)\right]_{2} \otimes D_{10}$ with index 2 and $\operatorname{End}(\bar{G}) \simeq \mathbb{Q}(\sqrt{-10})$. Then $E:=$ $\operatorname{End}(\bar{N}) \simeq \mathbb{Q}(\sqrt{-2}, \sqrt{5})$ and every $g \in G \backslash N$ acts on $E$ by $\sqrt{-2}^{g}=-\sqrt{-2}$ and $\sqrt{5}^{g}=-\sqrt{5}$. In particular, this rules out constructions (a) and (c) from Definition 2.4 .4 .

Let $\varphi: G_{1}:=\sqrt{-2}\left[\mathrm{GL}_{2}(3)\right]_{2} \stackrel{\sim}{\rightarrow}\left\langle\mathrm{SL}_{2}(3),\left(\begin{array}{cc}1 & 0 \\ 0 & -1\end{array}\right)\right\rangle \subset \mathbb{F}_{3}^{2 \times 2}$ and set $z:=\varphi^{-1}\left(\left(\begin{array}{cc}1 & 0 \\ 0 & -1\end{array}\right)\right)$. Further let $G_{2}:=D_{10}$ be generated by some $a, b \in N$ that satisfy $a^{5}=b^{2}=(a b)^{2}=1$.

Since $\operatorname{Out}\left(G_{i}\right) \simeq C_{2}$ we find some $g \in G$ which satisfies $a^{g}=g^{2}=b, z^{g}=-z$ and $g \in C_{G}\left(\mathcal{B}^{o}\left(O_{2}(G)\right)\right)$ where $\mathcal{B}^{o}\left(O_{2}(G)\right) \simeq \mathrm{SL}_{2}(3)$. (Note that we might have to exchange $b$ by $z b)$.

Now suppose $g=p^{-1} a_{1} a_{2} x$ as described in Definition 2.4.4 (b). Then

$$
b=g^{2}=p^{-2} a_{1} a_{2} x^{2} a_{1}^{x} a_{2}^{x}= \pm p^{-2}\left(a_{1} a_{1}^{x}\right)\left(a_{2} a_{2}^{x}\right)
$$

since the $a_{i} \in \overline{G_{i}}$ commute and $x^{2}= \pm 1$ by our assumption. The action of $x$ on $\overline{G_{2}}$ is explicitly known and one finds (using Groebner bases in MAGMA) that $a_{2}:=$ $\left(1+a^{2}+a^{3}\right)+b$ satisfies $a_{2} a_{2}^{x}=b$. In particular, if we set $p=a_{1}=1$ and $x:=a_{2}^{-1} \cdot g$, then $x^{2}=1$. One easily checks that these elements meet all requirements and thus $G \simeq \sqrt{-10}\left[\sqrt{-2}\left[\mathrm{GL}_{2}(3)\right]_{2} \stackrel{2}{\otimes} D_{10}\right]_{8}$ (see Theorem 4.9 .1 . 


\subsection{Some tables}

\subsubsection{Candidates for the layer}

Table 2.5.1 This table lists all quasisimple finite rational irreducible matrix groups $N$ in $\mathrm{GL}_{m}(\mathbb{Q})$ for $2 \leq m \leq 22$.

The representations with $m \geq 12$ and Schur index + are omitted since they can not be a subgroup of $E(G)$ for some s.p.i.m.f. subgroup $G \leq \operatorname{Sp}_{2 n}(\mathbb{Q})$ for $1 \leq n \leq 11$.

\begin{tabular}{|c|c|c|c|c|c|}
\hline$N$ & $\mathcal{B}^{o}(N)$ & $m$ & character & $\overline{\operatorname{End}(\bar{N})}$ & omit \\
\hline $\mathrm{Alt}_{5}$ & $\pm S_{5}=A_{4}$ & 4 & $\chi_{4}$ & $\mathbb{Q}$ & - \\
\hline $\mathrm{Alt}_{5}$ & $\pm S_{6}=A_{5}$ & 5 & $\chi_{5}$ & $\mathbb{Q}$ & + \\
\hline $\mathrm{Alt}_{6}$ & $\pm S_{6}=A_{5}$ & 5 & $\chi_{5 a}$ or $\chi_{5 b}$ & $\mathbb{Q}$ & - \\
\hline $\mathrm{Alt}_{5}$ & $\pm \mathrm{Alt}_{5}$ & 6 & $\chi_{3 a b}$ & $\mathbb{Q}(\sqrt{5})$ & - \\
\hline $\mathrm{L}_{2}(7)$ & $\pm \mathrm{L}_{2}(7)$ & 6 & $\chi_{3 a b}$ & $\mathbb{Q}(\sqrt{-7})$ & - \\
\hline $\mathrm{L}_{2}(7)$ & $\pm \mathrm{L}_{2}(7)$ & 6 & $\chi_{6}$ & $\mathbb{Q}$ & - \\
\hline $\mathrm{Alt}_{7}$ & $\pm S_{7}=A_{6}$ & 6 & $\chi_{6}$ & $\mathbb{Q}$ & - \\
\hline$U_{4}(2)$ & $\pm U_{4}(2): 2=E_{6}$ & 6 & $\chi_{6}$ & $\mathbb{Q}$ & - \\
\hline Alt $_{8}$ & $\pm S_{8}=A_{7}$ & 7 & $\chi_{7}$ & $\mathbb{Q}$ & - \\
\hline$U_{3}(3)$ & $\pm S_{6}(2)=E_{7}$ & 7 & $\chi_{7}$ & $\mathbb{Q}$ & + \\
\hline $\mathrm{L}_{2}(7)$ & $\pm S_{6}(2)=E_{7}$ & 7 & $\chi_{7}$ & $\mathbb{Q}$ & + \\
\hline $\mathrm{L}_{2}(8)$ & $\pm S_{6}(2)=E_{7}$ & 7 & $\chi_{7 a}$ & $\mathbb{Q}$ & + \\
\hline$S_{6}(2)$ & $\pm S_{6}(2)=E_{7}$ & 7 & $\chi_{7}$ & $\mathbb{Q}$ & - \\
\hline $\mathrm{SL}_{2}(5)$ & $\mathrm{SL}_{2}(5)$ & 8 & $2 \chi_{2 a b}$ & $\mathcal{Q}_{\sqrt{5}, \infty}$ & - \\
\hline $\mathrm{SL}_{2}(5)$ & $\mathrm{SL}_{2}(9)$ & 8 & $2 \chi_{4}$ & $\mathcal{Q}_{\infty, 3}$ & + \\
\hline $\mathrm{L}_{2}(7)$ & $\pm \mathrm{L}_{2}(7) .2=M_{8,3}$ & 8 & $\chi_{8}$ & $\mathbb{Q}$ & - \\
\hline $\mathrm{SL}_{2}(7)$ & $\mathrm{SL}_{2}(7)$ & 8 & $\chi_{4 a b}$ & $\mathbb{Q}(\sqrt{-7})$ & - \\
\hline $\mathrm{SL}_{2}(9)$ & $\mathrm{SL}_{2}(9)$ & 8 & $2 \chi_{4 a}$ or $2 \chi_{4 b}$ & $\mathcal{Q}_{\infty, 3}$ & - \\
\hline $\mathrm{L}_{2}(8)$ & $2 . O_{8}^{+}(2) .2=E_{8}$ & 8 & $\chi_{8}$ & $\mathbb{Q}$ & + \\
\hline $2 . \mathrm{Alt}_{7}$ & 2.Alt & 8 & $\chi_{4 a b}$ & $\mathbb{Q}(\sqrt{-7})$ & - \\
\hline 2. Alt $_{8}$ & $2 . O_{8}^{+}(2) .2=E_{8}$ & 8 & $\chi_{8}$ & $\mathbb{Q}$ & + \\
\hline $\mathrm{Sp}_{4}(3)$ & $\mathrm{Sp}_{4}(3) \circ C_{3}$ & 8 & $\chi_{4 a b}$ & $\mathbb{Q}(\sqrt{-3})$ & - \\
\hline Alt $_{9}$ & $2 . O_{8}^{+}(2) .2=E_{8}$ & 8 & $\chi_{8}$ & $\mathbb{Q}$ & + \\
\hline 2. Alt $_{9}$ & $2 . O_{8}^{+}(2) .2=E_{8}$ & 8 & $\chi_{8}$ & $\mathbb{Q}$ & + \\
\hline 2. $\mathrm{Sp}_{6}(2)$ & $2 . O_{8}^{+}(2) .2=E_{8}$ & 8 & $\chi_{8}$ & $\mathbb{Q}$ & + \\
\hline $2 . O_{8}^{+}(2)$ & $2 . O_{8}^{+}(2) .2=E_{8}$ & 8 & $\chi_{8}$ & $\mathbb{Q}$ & - \\
\hline $\mathrm{Alt}_{6}$ & $\pm S_{10}=A_{9}$ & 9 & $\chi_{9}$ & $\mathbb{Q}$ & + \\
\hline $\mathrm{Alt}_{10}$ & $\pm S_{10}=A_{9}$ & 9 & $\chi_{9}$ & $\mathbb{Q}$ & - \\
\hline $\mathrm{L}_{2}(11)$ & $\pm \mathrm{L}_{2}(11)$ & 10 & $\chi_{5 a b}$ & $\mathbb{Q}(\sqrt{-11})$ & - \\
\hline$U_{4}(2)=S_{4}(3)$ & $\pm S_{4}(3) \circ C_{3}$ & 10 & $\chi_{5 a b}$ & $\mathbb{Q}(\sqrt{-3})$ & - \\
\hline $\mathrm{L}_{2}(11)$ & $\pm \mathrm{L}_{2}(11): 2=A_{10}^{(2)}$ & 10 & $\chi_{10 a}$ & $\mathbb{Q}$ & - \\
\hline $\mathrm{L}_{2}(11)$ & $\pm \mathrm{L}_{2}(11)$ & 10 & $\chi_{10 b}$ & $\mathbb{Q}$ & - \\
\hline Alt $_{6}$ & $\pm S_{6}$ & 10 & $\chi_{10}$ & $\mathbb{Q}$ & - \\
\hline $\mathrm{Alt}_{11}$ & $\pm S_{11}=A_{10}$ & 10 & $\chi_{10}$ & $\mathbb{Q}$ & - \\
\hline$M_{11}$ & $\pm S_{11}=A_{10}$ & 10 & $\chi_{10 a}$ & $\mathbb{Q}$ & + \\
\hline
\end{tabular}




\begin{tabular}{|c|c|c|c|c|c|}
\hline $\mathrm{Alt}_{12}$ & $\pm S_{12}=A_{11}$ & 11 & $\chi_{11}$ & $\mathbb{Q}$ & - \\
\hline$M_{11}$ & $\pm M_{11}$ & 11 & $\chi_{11}$ & $\mathbb{Q}$ & - \\
\hline $\mathrm{L}_{2}(11)$ & $\pm S_{12}=A_{11}$ & 11 & $\chi_{11}$ & $\mathbb{Q}$ & + \\
\hline$M_{12}$ & $\pm S_{12}=A_{11}$ & 11 & $\chi_{11 a}$ or $\chi_{11 b}$ & $\mathbb{Q}$ & + \\
\hline $\mathrm{SL}_{2}(5)$ & $\mathrm{SL}_{2}(5)$ & 12 & $2 \chi_{6}$ & $\mathcal{Q}_{\infty, 2}$ & - \\
\hline $6 . U_{4}(3)$ & $6 . U_{4}(3) .2$ & 12 & $\chi_{12 a b}$ & $\mathbb{Q}(\sqrt{-3})$ & - \\
\hline 3. Alt $_{6}$ & \pm 3. Alt $_{6}$ & 12 & $\chi_{3 a b}+\chi_{3 a b}^{\prime}$ & $\mathbb{Q}(\sqrt{-3}, \sqrt{5})$ & - \\
\hline 3. Alt $_{6}$ & \pm 3. Alt $_{6}$ & 12 & $\chi_{6}+\chi_{6}^{\prime}$ & $\mathbb{Q}(\sqrt{-3})$ & - \\
\hline $\mathrm{SL}_{2}(11)$ & $\mathrm{SL}_{2}(11)$ & 12 & $\chi_{6 a b}$ & $\mathbb{Q}(\sqrt{-11})$ & - \\
\hline $3 . \mathrm{Alt}_{7}$ & $6 . U_{4}(3) .2$ & 12 & $\chi_{6}+\chi_{6}^{\prime}$ & $\mathbb{Q}(\sqrt{-3})$ & + \\
\hline$U_{3}(3)$ & $\pm U_{3}(3)$ & 12 & $2 \chi_{6}$ & $\mathcal{Q}_{\infty, 3}$ & - \\
\hline $6 . L_{4}(3)$ & $6 . L_{4}(3)$ & 12 & $\chi_{6}+\chi_{6}^{\prime}$ & $\mathbb{Q}(\sqrt{-3})$ & - \\
\hline$U_{3}(3)$ & $U_{3}(3) \circ C_{4}$ & 14 & $\chi_{7 a b}$ & $\mathbb{Q}(i)$ & - \\
\hline $\mathrm{SL}_{2}(7)$ & $\mathrm{SL}_{2}(7)$ & 16 & $2 \chi_{8}$ & $\mathcal{Q}_{\infty, 3}$ & - \\
\hline $\mathrm{L}_{2}(17)$ & $\pm \mathrm{L}_{2}(17) .2$ & 16 & $\chi_{16}$ & $\mathbb{Q}$ & - \\
\hline $\mathrm{L}_{2}(16)$ & $\pm S_{17}$ & 16 & $\chi_{16}$ & $\mathbb{Q}$ & + \\
\hline 2. Alt $_{10}$ & 2. Alt $_{10}$ & 16 & $\chi_{16}$ & $\mathbb{Q}$ & - \\
\hline $\mathrm{Alt}_{17}$ & $\pm S_{17}$ & 16 & $\chi_{16}$ & $\mathbb{Q}$ & - \\
\hline 3. Alt $_{6}$ & $\pm 3 . M_{10}$ & 18 & $\chi_{9 a b}$ & $\mathbb{Q}(\sqrt{-3})$ & - \\
\hline $\mathrm{L}_{2}(19)$ & $\pm \mathrm{L}_{2}(19)$ & 18 & $\chi_{9 a b}$ & $\mathbb{Q}(\sqrt{-19})$ & - \\
\hline $\mathrm{SL}_{2}(11)$ & $\mathrm{SL}_{2}(11)$ & 20 & $2 \chi_{10}$ & $\mathcal{Q}_{\infty, 2}$ & - \\
\hline $\mathrm{Alt}_{7}$ & $\pm \mathrm{Alt}_{7}$ & 20 & $\chi_{10 a b}$ & $\mathbb{Q}(\sqrt{-7})$ & - \\
\hline$M_{11}$ & $2 . M_{12}: 2$ & 20 & $\chi_{10 b c}$ & $\mathbb{Q}(\sqrt{-2})$ & + \\
\hline $2 . M_{12}$ & $2 . M_{12}: 2$ & 20 & $\chi_{10 a b}$ & $\mathbb{Q}(\sqrt{-2})$ & - \\
\hline $2 . M_{22}$ & $2 . M_{22}: 2$ & 20 & $\chi_{10 a b}$ & $\mathbb{Q}(\sqrt{-7})$ & - \\
\hline$U_{4}(2)$ & $\pm U_{4}(2) \circ C_{3}$ & 20 & $\chi_{10 a b}$ & $\mathbb{Q}(\sqrt{-3})$ & - \\
\hline$U_{5}(2)$ & $\pm U_{5}(2)$ & 20 & $2 \chi_{10}$ & $\mathcal{Q}_{\infty, 2}$ & - \\
\hline $2 . L_{3}(4)$ & 2. $L_{3}(4): 2_{2}$ & 20 & $\chi_{10 a b}$ & $\mathbb{Q}(\sqrt{-7})$ & - \\
\hline $\mathrm{SL}_{2}(19)$ & $\mathrm{SL}_{2}(19)$ & 20 & $\chi_{10 a b}$ & $\mathbb{Q}(\sqrt{-19})$ & - \\
\hline$U_{5}(2)$ & $\pm U_{5}(2) \circ C_{3}$ & 22 & $\chi_{11 a b}$ & $\mathbb{Q}(\sqrt{-3})$ & - \\
\hline $\mathrm{L}_{2}(23)$ & $\pm \mathrm{L}_{2}(23)$ & 22 & $\chi_{11 a b}$ & $\mathbb{Q}(\sqrt{-23})$ & - \\
\hline
\end{tabular}

If $N$ is not normal in $\mathcal{B}^{\circ}(N)$, then $N$ is not a normal subgroup of any s.p.i.m.f. matrix group (see Lemma 2.1.23). These cases are indicated in the last row with $a+$.

Note also that the above characters are indexed by their degrees and not the numbers given in the ATLAS [CCN $\left.{ }^{+} 85\right]$.

Proof: This table can be taken from [Neb98, Table 9.1]. Alternatively, the representations can be taken from [Nic06] which is based on [HM01]. For the generalized Bravais groups, one computes the order $\Lambda_{\infty}(N) \subset \mathbb{Q}^{m \times m}$ (cf. Section 2.1.3) using linear algebra over $\mathbb{Z}$. Then one computes some $\Lambda_{\infty}(N)$-invariant lattices and takes the intersection $A$ of their automorphism groups wrt. the full form space $\mathcal{F}(N)$. If $N \unlhd A$ then $\mathcal{B}^{o}(N)=A$. Otherwise, one computes $\mathcal{B}^{\circ}(N)$ using the isomorphism classes of $\mathcal{Z}\left(\Lambda_{\infty}(N)\right)$ using Remark 2.3.2. This method works for all the groups $N$ from above except $\mathrm{SL}_{2}(5)$ with character $2 \chi_{4}$ since here $N \nsubseteq \mathcal{B}^{\circ}(N)$ and $\operatorname{End}(\bar{N})$ is not a field. 
In this case, one can use the algorithm [Neb95, III.11] to compute the isomorphism classes of $\Lambda_{\infty}(N)$-invariant lattices.

\subsubsection{Candidates for the Fitting subgroup}

Table 2.5.2 All candidates for $N:=O_{p}(G)$ of an irreducible symplectic primitive matrix group $G$ are given by

\begin{tabular}{|l|c|c|c|}
\hline$N$ & $\mathcal{B}^{o}(N)$ & $\operatorname{dim}_{\mathbb{Q}}(\bar{N})$ & $\operatorname{End}(\bar{N})$ \\
\hline$C_{p^{m}}$ & $\pm N$ & $p^{m-1}(p-1)$ & $\mathbb{Q}\left(\zeta_{p^{m}}\right)$ \\
\hline$p_{+}^{1+2 n}, \quad(p>2)$ & $\pm N . \operatorname{Sp}_{2 n}(p)$ & $p^{n}(p-1)$ & $\mathbb{Q}\left(\zeta_{p}\right)$ \\
\hline $2_{+}^{1+2 n}$ & $N . O_{2 n}^{+}(2)$ & $2^{n}$ & $\mathbb{Q}$ \\
\hline $2_{-}^{1+2 n}$ & $N . O_{2 n}^{-}(2)$ & $2^{n+1}$ & $\mathcal{Q}_{\infty, 2}$ \\
\hline$p_{+}^{1+2 n} Y C_{p^{m}}, \quad(m>1)$ & $\pm N . \operatorname{Sp}_{2 n}(p)$ & $p^{m+n-1}(p-1)$ & $\mathbb{Q}\left(\zeta_{p}\right)$ \\
\hline $2_{+}^{1+2 n} \curlyvee D_{2^{m}}, \quad(m>3)$ & $N . \operatorname{Sp}_{2 n}(2)$ & $2^{n+m-2}$ & $\mathbb{Q}\left(\theta_{2^{m-1}}\right)$ \\
\hline $2_{+}^{1+2 n} \curlyvee Q_{2^{m}}, \quad(m>3)$ & $N . \operatorname{Sp}_{2 n}(2)$ & $2^{n+m-1}$ & $\mathcal{Q}_{2_{2^{m-1}}, \infty}$ \\
\hline $2_{+}^{1+2 n} Y Q D_{2^{m}}, \quad(m>3)$ & $N . \operatorname{Sp}_{2 n}(2)$ & $2^{n+m-2}$ & $\mathbb{Q}\left(\zeta_{2^{m-1}}-\zeta_{2^{m-1}}^{-1}\right)$ \\
\hline
\end{tabular}

In the last three rows $n=0$ is allowed. In these cases $N$ is $D_{2^{m}}, Q_{2^{m}}$ or $Q D_{2^{m}}$ respectively.

Note that $\mathcal{B}^{\circ}(N)$ is only correct under the assumption that $N \unlhd G$.

Proof: All abelian characteristic subgroups of $N$ are cyclic by Corollary 2.1.16. Thus $N$ must be isomorphic to a subgroup from above by a theorem of $\mathrm{Ph}$. Hall (see Theorem 2.1.17). These groups have only one faithfull rational irreducible representation. The generalized Bravais groups $\mathcal{B}^{\circ}(N)$ of these representations have been computed in [Neb98, Chapter 8] (under the assumption that $N \unlhd G$ ).

\subsubsection{Tables for number fields}

Table 2.5.3 This table contains information on cyclotomic number fields that is needed to construct the s.i.m.f. supergroups of some irreducible cyclic subgroups of order $m$.

The second column contains the set $\mathcal{S}_{m}:=\tilde{\Pi}\left(\mathbb{Q}\left(\theta_{m}\right), m\right) \backslash \Pi(m)$ of "additional primes"; if not empty. The third column contains the decomposition of $p \mathbb{Z}\left[\theta_{m}\right]$ into prime ideals for various primes $p$. The last column contains generators for all minimal totally complex subfields of $\mathbb{Q}\left(\zeta_{m}\right)$.

The fourth column contains the narrow class group $\mathcal{C} l^{+}\left(\mathbb{Z}\left[\theta_{m}\right]\right)$; if not trivial. It is given as follows: Let $\psi_{m}: \mathcal{C} l^{+}\left(\mathbb{Z}\left[\theta_{m}\right]\right) \stackrel{\sim}{\rightarrow} \bigoplus_{i} \mathbb{Z} / a_{m, i} \mathbb{Z}$ be an isomorphism where the $a_{m, i}$ are elementary divisors. The first row lists the $a_{m, i}$. The following rows list the nonzero images of the prime ideals from above under $\psi_{m}$. The superscript $(*)$ means all ideals of a given norm. 


\begin{tabular}{|c|c|c|c|c|c|}
\hline$m$ & $\mathcal{S}_{m}$ & prime decomposition in $\mathbb{Z}\left[\theta_{m}\right]$ & $\mathcal{C} l^{+}\left(\mathbb{Z}\left[\theta_{r}\right.\right.$ & & $\begin{array}{l}\text { minimal totally complex } \\
\text { subfields of } \mathbb{Q}\left(\zeta_{m}\right)\end{array}$ \\
\hline 8 & & $(2)=\mathfrak{p}_{2}^{2},(3),(5)$ & & & $i, \sqrt{-2}$ \\
\hline \multirow{2}{*}{12} & & \multirow{2}{*}{$(2)=\mathfrak{p}_{2}^{2},(3)=\mathfrak{p}_{3}^{2},(5)$} & & 2 & \multirow{2}{*}{$i, \sqrt{-3}$} \\
\hline & & & \multirow{2}{*}{\multicolumn{2}{|c|}{$\mathfrak{p}_{2}, \mathfrak{p}_{3}$}} & \\
\hline 14 & & $(2),(3),(5),(7)=\mathfrak{p}_{7}^{3}$ & & & $\sqrt{-7}$ \\
\hline 16 & & $(2)=\mathfrak{p}_{2}^{4},(3),(5)$ & & & $i, \sqrt{-2}, \zeta_{16}-\zeta_{16}^{-1}$ \\
\hline 18 & & $(2),(3)=\mathfrak{p}_{3}^{3},(5),(7)$ & & & $\sqrt{-3}$ \\
\hline \multirow[t]{2}{*}{20} & & \multirow{2}{*}{$(2)=\mathfrak{p}_{2}^{2},(3),(5)=\mathfrak{p}_{5}^{4}$} & & 2 & \multirow{2}{*}{$i, \sqrt{-5}, \zeta_{10}$} \\
\hline & & & $\mathfrak{p}_{2}$ & 1 & \\
\hline \multirow[t]{2}{*}{24} & & \multirow{2}{*}{$(2)=\mathfrak{p}_{2}^{4},(3)=\mathfrak{p}_{3}^{2},(5)=\mathfrak{p}_{5} \mathfrak{p}_{5}^{\prime}$} & & \multirow{2}{*}{$\frac{2}{1} i$} & $i, \sqrt{-2}, \sqrt{-3}, \sqrt{-6}$ \\
\hline & & & $\mathfrak{p}_{2}$ & & \\
\hline \multirow[t]{2}{*}{28} & & \multirow{2}{*}{$(2)=\mathfrak{p}_{2}^{2},(3)=\mathfrak{p}_{3} \mathfrak{p}_{3}^{\prime},(5),(7)=\mathfrak{p}_{7}^{6}$} & & \multirow{2}{*}{\multicolumn{2}{|c|}{$\frac{2}{1} i, \sqrt{-7}$}} \\
\hline & & & $\mathfrak{p}_{3}, \mathfrak{p}_{7}$ & 1 & \\
\hline 30 & & $(2),(3)=\mathfrak{p}_{3}^{2},(5)=\mathfrak{p}_{5}^{4}$ & & \multirow{3}{*}{$\begin{array}{l}2 \\
1\end{array}$} & $\sqrt{-3}, \sqrt{-15}, \zeta_{10}$ \\
\hline 32 & & & $p_{3}, \uparrow_{5}$ & & $i \sqrt{-2} \zeta c-C^{-1}$ \\
\hline 32 & & $\begin{array}{l}(7)=\mathfrak{p}_{2},(13),(13) \\
(7)\end{array}$ & & & $\begin{array}{l}\zeta_{32}-\zeta_{32}^{-1} \\
\end{array}$ \\
\hline \multirow[t]{2}{*}{36} & & \multirow{2}{*}{$(2)=\mathfrak{p}_{2}^{2},(3)=\mathfrak{p}_{3}^{6},(5),(7)$} & & \multirow{2}{*}{\multicolumn{2}{|c|}{\begin{tabular}{l|l}
2 & $i, \sqrt{-3}$
\end{tabular}}} \\
\hline & & & $\mathfrak{p}_{2}, \mathfrak{p}_{3}$ & & \\
\hline \multirow{2}{*}{40} & & \multirow{2}{*}{$(2)=\mathfrak{p}_{2}^{4},(3)=\mathfrak{p}_{3} \mathfrak{p}_{3}^{\prime},(5)=\mathfrak{p}_{5}^{4}$} & & \multirow{2}{*}{\multicolumn{2}{|c|}{$\begin{array}{l}i, \sqrt{-2}, \sqrt{-5}, \sqrt{-10} \\
\zeta_{10}, \sqrt{2} \cdot\left(\zeta_{10}-\zeta_{10}^{-1}\right)\end{array}$}} \\
\hline & & & $\mathfrak{p}_{2}$ & 1 & \\
\hline \multirow{2}{*}{42} & & \multirow{2}{*}{$(2),(3)=\mathfrak{p}_{3}^{2},(5)=\mathfrak{p}_{5} \mathfrak{p}_{5}^{\prime},(7)=\mathfrak{p}_{7}^{6}$} & & \multirow{2}{*}{\multicolumn{2}{|c|}{$\sqrt{-3}, \sqrt{-7}$}} \\
\hline & & & $\mathfrak{p}_{3}, \mathfrak{p}_{5}^{(*)}$ & 1 & \\
\hline \multirow{2}{*}{44} & & \multirow{2}{*}{$\begin{array}{l}(2)=\mathfrak{p}_{2}^{2},(3),(5)=\mathfrak{p}_{5} \mathfrak{p}_{5}^{\prime} \\
(11)=\mathfrak{p}_{11}^{10}\end{array}$} & & \multirow{2}{*}{\multicolumn{2}{|c|}{$i, \sqrt{-11}$}} \\
\hline & & & $\mathfrak{p}_{2}, \mathfrak{p}_{11}$ & 1 & \\
\hline \multirow{2}{*}{48} & & \multirow{2}{*}{$(2)=\mathfrak{p}_{2}^{8},(3)=\mathfrak{p}_{3}^{2}$} & & 2 & $i, \sqrt{-2}, \sqrt{-3}, \sqrt{-6}$ \\
\hline & & & $\mathfrak{p}_{2}$ & 1 & $\sqrt{3} \cdot\left(\zeta_{16}-\zeta_{16}^{-1}\right), \zeta_{16}-\zeta_{16}^{-1}$ \\
\hline 50 & & $(2),(3),(5)=\mathfrak{p}_{5}^{10}$ & & & $\zeta_{10}$ \\
\hline 54 & & $(2),(3)=\mathfrak{p}_{3}^{9},(5),(7),(11),(13)$ & & & $\sqrt{-3}$ \\
\hline 60 & $\{1150\}$ & $(2)=\mathfrak{p}_{2}^{2},(3)=\mathfrak{p}_{3}^{2},(5)=\mathfrak{p}_{5}^{4}$ & & 2 & $i, \sqrt{-3}, \sqrt{-5}, \sqrt{-15}$ \\
\hline 00 & $\{11,59\}$ & $(11)=\mathfrak{p}_{11} \mathfrak{p}_{11}^{\prime} \mathfrak{p}_{11}^{\prime \prime} \mathfrak{p}_{11}^{\prime \prime \prime}$ & - & 1 & $\zeta_{10}, \sqrt{3} \cdot\left(\zeta_{10}-\zeta_{10}^{-1}\right)$ \\
\hline 66 & & $(2)=\mathfrak{p}_{2} \mathfrak{p}_{2}^{\prime},(3)=\mathfrak{p}_{3}^{2},(5),(7)$, & & 2 & $3, \sqrt{-}$ \\
\hline 00 & & $(11)=\mathfrak{p}_{11}^{10}$ & $\mathfrak{p}_{2}^{(*)}, \mathfrak{p}_{11}$ & 1 & \\
\hline
\end{tabular}

All cyclotomic fields $\mathbb{Q}\left(\zeta_{m}\right)$ from above have class number 1 . This implies that their maximal totally real subfields $\mathbb{Q}\left(\theta_{m}\right)$ also have class number 1 (see Was96. Theorem 4.10]). Moreover $\operatorname{Nr}_{\mathbb{Q}\left(\zeta_{m}\right) / \mathbb{Q}\left(\theta_{m}\right)}\left(\mathbb{Z}\left[\zeta_{m}\right]^{*}\right)=\mathbb{Z}\left[\theta_{m}\right]_{>0}^{*}$ in all cases. So one only has to consider a single class of totally positive units in the $m$-parameter argument (see Remark 2.2.13).

Proof: Follows from explicit calculations with MAGMA [BCP97]. 
Table 2.5.4 The following table contains information on some totally real number fields $K$ needed for the m-parameter argument in the sequel. The second column contains the set $\tilde{\Pi}(K, 2) \backslash\{2\}$. The fourth column describes the narrow class group $\mathcal{C l}^{+}\left(\mathbb{Z}_{K}\right)$ in the notation of the previous table.

\begin{tabular}{|c|c|c|c|c|}
\hline$K$ & & prime decomposition in $\mathbb{Z}_{K}$ & $\mathcal{C} l^{+}\left(\mathbb{Z}_{K}\right.$ & $\mathbb{Z}_{K,>0}^{*} /\left(\mathbb{Z}_{K}^{*}\right)^{2}$ \\
\hline $\mathbb{Q}(\sqrt{2})$ & $\emptyset$ & $(2)=\mathfrak{p}_{2}^{2},(3),(5)$ & 1 & 1 \\
\hline \multirow{2}{*}{$\mathbb{Q}(\sqrt{3})$} & \multirow{2}{*}{$\emptyset$} & \multirow{2}{*}{$(2)=\mathfrak{p}_{2}^{2},(3)=\mathfrak{p}_{3}^{2},(5),(7)$} & & \multirow{2}{*}{$C_{2}$} \\
\hline & & & $\mathfrak{p}_{2}, \mathfrak{p}_{3}$ & \\
\hline $\mathbb{Q}(\sqrt{5})$ & $\emptyset$ & $(2),(3),(5)=\mathfrak{p}_{5}^{2}$ & 1 & 1 \\
\hline \multirow{2}{*}{$\mathbb{Q}(\sqrt{6})$} & \multirow{2}{*}{$\emptyset$} & \multirow{2}{*}{$(2)=\mathfrak{p}_{2}^{2},(3)=\mathfrak{p}_{3}^{2},(5)=\mathfrak{p}_{5} \mathfrak{p}^{\prime}{ }_{5}$} & & \multirow{2}{*}{$C_{2}$} \\
\hline & & & $\mathfrak{p}_{2}, \mathfrak{p}_{5}^{(*)}$ & \\
\hline \multirow{2}{*}{$\mathbb{Q}(\sqrt{7})$} & \multirow{2}{*}{3} & \multirow{2}{*}{$(2)=\mathfrak{p}_{2}^{2},(3)=\mathfrak{p}_{3} \mathfrak{p}_{3}^{\prime},(5),(7)=\mathfrak{p}_{7}^{2}$} & & \multirow{2}{*}{$C_{2}$} \\
\hline & & & $\mathfrak{p}_{2}, \mathfrak{p}_{3}^{(*)}$ & \\
\hline \multirow{2}{*}{$\mathbb{Q}(\sqrt{21})$} & \multirow{2}{*}{3} & \multirow{2}{*}{$(2),(3)=\mathfrak{p}_{3}^{2},(5)=\mathfrak{p}_{5} \mathfrak{p}_{5}^{\prime},(7)=\mathfrak{p}_{7}^{2}$} & & \multirow{2}{*}{$C_{2}$} \\
\hline & & & $\mathfrak{p}_{3}, \mathfrak{p}_{5}^{(*)}$ & \\
\hline \multirow{2}{*}{$\mathbb{Q}\left(\sqrt{-2} \cdot\left(\zeta_{10}-\zeta_{10}^{-1}\right)\right)$} & \multirow{2}{*}{$\emptyset$} & \multirow{2}{*}{$(2)=\mathfrak{p}_{2}^{2},(3),(5)=\mathfrak{p}_{5}^{4}$} & & \multirow{2}{*}{$C_{2}$} \\
\hline & & & $\mathfrak{p}_{2}, \mathfrak{p}_{5}$ & \\
\hline $\mathbb{Q}(\sqrt{2}, \sqrt{5})$ & $\emptyset$ & $(2)=\mathfrak{p}_{2}^{2},(3)=\mathfrak{p}_{3} \mathfrak{p}_{3}^{\prime},(5)=\mathfrak{p}_{5}^{2}$ & 1 & 1 \\
\hline \multirow{2}{*}{$\mathbb{Q}(\sqrt{3}, \sqrt{5})$} & \multirow[t]{2}{*}{11} & \multirow{2}{*}{$(2)=\mathfrak{p}_{2}^{2},(3)=\mathfrak{p}_{3}^{2},(5)=\mathfrak{p}_{5}^{2}$} & & \multirow{2}{*}{$C_{2}$} \\
\hline & & & & \\
\hline
\end{tabular}

Proof: Follows from explicit calculations with MAGMA [BCP97]. 


\section{Chapter 3}

\section{Some infinite s.i.m.f. families}

\subsection{Some subgroups of $\operatorname{Sp}_{p-1}(\mathbb{Q})$}

Let $p \geq 5$ be a prime and write $p-1=2^{a} \cdot o$ with $o$ odd. In the spirit of [NP95, chapter V] we describe all s.i.m.f. supergroups $G$ of $C_{p}$ in dimension $p-1$ where $C_{p}$ denotes the (up to conjugacy) unique cyclic matrix group of order $p$ in $\mathrm{GL}_{p-1}(\mathbb{Q})$.

Clearly, one possibility is that $G$ contains a normal subgroup conjugate to $C_{p}$. Since the commuting algebra of $C_{p}$ is isomorphic to $\mathbb{Q}\left(\zeta_{p}\right)$ we have $G / \pm C_{p} \leq C_{2^{a}} \times C_{o}$. By Galois theory, $G$ is symplectic if and only if $G / \pm C_{p} \leq C_{o}$ and therefore $G \simeq \pm C_{p}: C_{o}$ by maximality. (The group $\pm C_{p}: C_{o}$ has only one irreducible rational representation of degree $p-1$ and we identify the group with this representation.)

Another class of candidates are extensions of $\mathrm{L}_{2}(p)$. The smallest faithful irreducible complex representations of $\mathrm{L}_{2}(p)$ are of degree $\frac{p-1}{2}$ and algebraically conjugate. The corresponding character field is $\mathbb{Q}(\sqrt{ \pm p})$ with the - sign if and only if $p \equiv_{4}-1$ (see [Dor71, §38]).

If $p \equiv_{4}-1$ then $\mathrm{L}_{2}(p)$ contains a subgroup $U$ isomorphic to $C_{p}: C_{\frac{p-1}{2}}$. The restriction of the natural representation of $\mathrm{L}_{2}(p)$ on $U$ is irreducible and has the same character field $\mathbb{Q}(\sqrt{-p})([$ Dor71, $\S 38])$. By [Lor71, Satz 1.2.1, p. 67], the Schur index of $\mathrm{L}_{2}(p)$ is equal to the Schur index of $U$ which is 1 . Thus $C_{2} \times \mathrm{L}_{2}(p)$ has a unique $p-1$ dimensional rationally irreducible representation (denoted by $\sqrt{-p}\left[ \pm \mathrm{L}_{2}(p)\right]_{\frac{p-1}{2}}$ in the sequel) with commuting algebra $\mathbb{Q}(\sqrt{-p})$.

The next result shows that there are no further possibilites. More precisely:

Theorem 3.1.1 Let $p \geq 11$ be prime and $G<\operatorname{Sp}_{p-1}(\mathbb{Q})$ such that $p$ divides $|G|$. Write $p-1=2^{a} \cdot$ o with o odd.

Then $G$ is s.i.m.f. if and only if $G$ is conjugate to $\left\{\begin{array}{ll} \pm C_{p}: C_{o} & \text { if } p \equiv_{4}+1 \\ \sqrt{-p}\left[ \pm \mathrm{L}_{2}(p)\right]_{\frac{p-1}{2}} & \text { if } p \equiv_{4}-1\end{array}\right.$.

Proof: Let $\pm I_{p-1}<G<\operatorname{Sp}_{p-1}(\mathbb{Q})$ such that $p$ divides $|G|$. Further let $P \in \operatorname{Syl}_{p}(G)$. Then by Minkowski's bound, $|P|=p$. So the commuting algebra $E$ of $P$ is isomorphic to $\mathbb{Q}\left(\zeta_{p}\right)$. Thus $\pm I_{p-1} \leq Z(G) \leq C_{G}(P)= \pm P$. Since $G$ must have a faithful 
irreducible complex character of degree $\leq \frac{p-1}{2}$ it follows from a theorem of $\mathrm{H}$. Blau [Fei82, VIII Theorem 7.2, p. 365] that either $P \unlhd G$ or $G / Z(G) \simeq \mathrm{L}_{2}(p)$. In the first case, $G$ is conjugate to a subgroup of $\pm C_{p}: C_{o}$. In the second case, $Z(G)= \pm P$ would imply that $G= \pm P$ since $\pm P$ is self-centralizing in $G$. So $Z(G)= \pm I_{p-1}$. Since $\mathrm{L}_{2}(p)$ is perfect, $G$ is either isomorphic to $\pm \mathrm{L}_{2}(p)$ or $\mathrm{SL}_{2}(p)$. So by [Dor71, Th. 38.1] this implies $p \equiv_{4}-1$. But if $p \equiv_{4}-1$ the real Schur indices of the $\frac{p-1}{2}$ dimensional complex characters of $\mathrm{SL}_{2}(p)$ are 2 (which can be computed from the character table of $\left.\mathrm{SL}_{2}(p)\right)$. Thus $G$ is conjugate to $\sqrt{-p}\left[ \pm \mathrm{L}_{2}(p)\right]_{\frac{p-1}{2}}$. Since $\sqrt{-p}\left[ \pm \mathrm{L}_{2}(p)\right]_{\frac{p-1}{2}}$ contains a subgroup conjugate to $\pm C_{p}: C_{o}$ the result follows.

Note that the above result is also true for $p=5$ and 7 as the explicit calculations in Lemmas 4.3 .3 and 4.4 .2 show.

\subsection{Some subgroups of $\operatorname{Sp}_{p+1}(\mathbb{Q})$}

Let $p \geq 5$ be a prime. If $p \equiv_{4}-1$ then $G:=\mathrm{SL}_{2}(p)$ has only two algebraically conjugate complex representations of degree $\frac{p+1}{2}$ as the generic character table [Dor71, Th. 38.1] shows. Let $\chi$ denote one of the corresponding characters and let $P \in \operatorname{Syl}_{p}(G)$. An explicit calculation shows $\left(1_{P}^{G}, \chi\right)_{G}=\left(1_{P},\left.\chi\right|_{P}\right)_{P}=1$. Thus (by Isa94, Corollary (10.2) part (c)]) $\chi$ is realizable over its character field, which is $\mathbb{Q}(\sqrt{-p})$. So $\chi$ gives rise to a subgroup of $\mathrm{Sp}_{p+1}(\mathbb{Q})$ denoted by $\sqrt{-p}\left[\mathrm{SL}_{2}(p)\right]_{\frac{p+1}{2}}$.

Theorem 3.2.1 Let $p \geq 11$ be prime and $G<\operatorname{Sp}_{p+1}(\mathbb{Q})$ such that $p$ divides $|G|$. Then $G$ is s.i.m.f. if and only if $p \equiv_{4}-1$ and $G$ is conjugate to $\sqrt{-p}\left[\mathrm{SL}_{2}(p)\right]_{\frac{p+1}{2}}$.

Proof: Let $P \in \operatorname{Syl}_{p}(G)$. Then again, by Minkowski's bound, $|P|=p$. Thus the natural representation of $P$ splits into twice the trivial one and the $\mathbb{Q}$-irreducible of degree $p-1$. Since there exists an embedding $\delta: G \rightarrow \mathrm{GL}_{m}(K)$ for some totally complex number field $K$ with $m \cdot[K: \mathbb{Q}]=p+1$ there is only the possibility $K=$ $\mathbb{Q}(\sqrt{-p})$ and $m=\frac{p+1}{2}$. So the commuting algebra of $\delta(P)$ in $K^{m \times m}$ is isomorphic to $\mathbb{Q}\left(\zeta_{p}\right) \times \mathbb{Q}(\sqrt{-p})$. In particular, $Z(G)$ is isomorphic to a subgroup of $C_{2 p} \times C_{2}$. By Corollary 2.1.16 we know that $P \notin G$ and therefore $Z(G) \leq C_{2} \times C_{2}$. Since $G$ cannot be imprimitive by Minkowski's bound, $Z(G)$ is not isomorphic to $C_{2} \times C_{2}$. This shows $Z(G)= \pm I_{p+1}$. From Blau's theorem [Fei82, VIII Theorem 7.2, p. 365] it follows that $G$ must be isomorphic to $\pm \mathrm{L}_{2}(p)$ or $\mathrm{SL}_{2}(p)$. Since $K \simeq \mathbb{Q}(\sqrt{-p})$ it follows from the character table of $\mathrm{SL}_{2}(p)$ [Dor71, Th. 38.1] that $p \equiv_{4}-1$ and that $\pm \mathrm{L}_{2}(p)$ has no faithful complex character of degree $\frac{p+1}{2}$. So $G$ must be conjugate to $\sqrt{-p}\left[\mathrm{SL}_{2}(p)\right]_{\frac{p+1}{2}}$ and the result follows.

Remark 3.2.2 Theorem 4.4.1 shows that the above result also holds for $p=5$. But according to Theorem 4.5.1, the unique s.i.m.f. subgroup of $\operatorname{Sp}_{8}(\mathbb{Q})$ whose order is divisible by 7 is $\sqrt{-7}\left[2 . \mathrm{Alt}_{7}\right]_{4}$ (which contains a subgroup conjugate to $\mathrm{SL}_{2}(7)$ ). 


\subsection{The group $Q D_{2^{n}}$}

Let $n \geq 4$. The group $Q D_{2^{n}}=\left\langle x, y \mid x^{2^{n-1}}, y^{2}, x^{y}=x^{2^{n-2}-1}\right\rangle$ has one rationally irreducible representation of degree $2^{n-2}$. This representation has $\mathbb{Q}\left(\zeta_{2^{n-1}}-\zeta_{2^{n-1}}^{-1}\right)$ as commuting algebra, so we denote it by $\zeta_{2^{n-1}}-\zeta_{2^{n-1}}^{-1}\left[Q D_{2^{n}}\right]_{2}$.

Lemma 3.3.1 If $n \geq 5$ then $\zeta_{2^{n-1}-\zeta_{2^{n-1}}^{-1}}\left[Q D_{2^{n}}\right]_{2}$ is a s.i.m.f. subggroup of $\mathrm{Sp}_{2^{n-2}}(\mathbb{Q})$.

Proof: The commuting algebra of $H:=\zeta_{2^{n-1}-\zeta_{2^{n-1}}^{-1}}\left[Q D_{2^{n}}\right]_{2}$ is isomorphic to $K:=$ $\mathbb{Q}\left(\zeta_{2^{n-1}}-\zeta_{2^{n-1}}^{-1}\right)$. This is the fixed field of the automorphism of $\mathbb{Q}\left(\zeta_{2^{n-1}}\right)$ induced by $\zeta_{2^{n-1}} \mapsto-\zeta_{2^{n-1}}^{-1}=\zeta_{2^{n-1}}^{2^{n-2}-1}$. The subfields of $K$ are linearly ordered by Galois theory and the maximal subfield is the fixed-field of complex conjugation i.e. totally real. Thus each s.i.m.f. supergroup $G$ of $H$ embeds into $G_{2}(K)$. Therefore $\bar{G}:=G / Z(G)=$ $G /\left\langle \pm I_{2^{n-2}}\right\rangle$ embeds into $\mathrm{PGL}_{2}(K)$. Since $n \geq 5$ is assumed we get $\bar{G}$ is a dihedral group (of order $2^{n-1}$ ) according to Blichfeldt's classification [Bli17]. This shows $G=H$ as claimed.

\subsection{The group $2_{+}^{1+2 n}$}

In this section, which is heavily based on Section 5 of [NRS01], let $T_{n} \simeq 2_{+}^{1+2 n}$ be the $n$-fold tensor product of $\left\langle\left(\begin{array}{ll}0 & 1 \\ 1 & 0\end{array}\right),\left(\begin{array}{cc}1 & 0 \\ 0 & -1\end{array}\right)\right\rangle \simeq D_{8}$. We describe the construction of $\mathcal{B}^{\circ}\left(T_{n}\right)$ and define a maximal finite subgroup of $\mathrm{GL}_{2^{n}}(\mathbb{Q}(\sqrt{-2}))$.

Let $\left(b_{0}, \ldots, b_{2^{n}-1}\right)$ be the standard basis of $\mathbb{Q}^{1 \times 2^{n}}$. We identify $v \in \mathbb{F}_{2}^{n}$ with $j=$ $\sum_{i} v_{i} 2^{i-1}$ and thereby we index the basis vectors $b_{j}$ with elements of $\mathbb{F}_{2}^{n}$. For an affine subspace $U$ of $\mathbb{F}_{2}^{n}$ let $\chi_{U}=\sum_{u \in U} b_{u}$. Then $L_{n}$ and $L_{n}^{\prime}$ are the $\mathbb{Z}$-lattices in $\mathbb{Q}^{1 \times 2^{n}}$ spanned by

$$
\left\{2^{\lfloor(n-\operatorname{dim}(U)+\delta) / 2\rfloor} \chi_{U} \mid U \text { an affine subspace of } \mathbb{F}_{2}^{n}\right\}
$$

where $\delta=0$ for $L_{n}$ and $\delta=1$ for $L_{n}^{\prime}$.

In [Wal62, Theorem 3.2] it is shown that $H_{n}:=\operatorname{Aut}\left(L_{n}, I_{2^{n}}\right) \cap \operatorname{Aut}\left(L_{n}^{\prime}, I_{2^{n}}\right)$ is isomorphic to $2_{+}^{1+2 n} \cdot O_{2 n}^{+}(2)$ and further $O_{2}\left(H_{n}\right)$ is conjugate to $T_{n}$.

By [Win72] we have $\operatorname{Out}\left(2_{+}^{1+2 n}\right) \simeq O_{2 n}^{+}(2): 2 \simeq G O_{2 n}^{+}(2)$ the full orthogonal group of a quadratic form of Witt defect 0 . Conjugation by $h_{n}:=\left(\begin{array}{cc}1 & 1 \\ 1 & -1\end{array}\right) \otimes I_{2^{n-1}}$ induces an outer automorphism on $T_{n}$ which is not realized by $H_{n}$. Since $\operatorname{End}\left(\bar{T}_{n}\right) \simeq \mathbb{Q}$ and $h_{n}^{2}=2 I_{2^{n}}$, there exists no element in $\mathrm{GL}_{2^{n}}(\mathbb{Q})$ of finite order which induces the same automorphism on $T_{n}$. Thus $H_{n}$ is conjugate to $\mathcal{B}^{o}\left(T_{n}\right)$. Moreover $\frac{1}{\sqrt{-2}} h_{n}$ normalizes $T_{n}$ and therefore $\mathcal{B}^{o}\left(T_{n}\right)$. Hence $\mathcal{H}_{n}:=\left\langle H_{n}, \frac{1}{\sqrt{-2}} h_{n}\right\rangle$ is the unique extension of $H_{n}$ in $\mathrm{GL}_{2^{n}}(\mathbb{Q}(\sqrt{-2}))$. The group $\mathcal{H}_{n}$ gives rise to a finite symplectic matrix group in $\mathrm{Sp}_{2^{n+1}}(\mathbb{Q})$ which will be denoted by $\sqrt{-2}\left[2_{+}^{1+2 n} \cdot\left(O_{2 n}^{+}(2): 2\right)\right]_{2^{n}}$ in the sequel. 
Finally, by extending scalars $M_{n}:=\sqrt{-2} L_{n}^{\prime}+L_{n}$ is a $\mathbb{Z}[\sqrt{-2}]$-lattice generated by

$$
\left\{\sqrt{-2}^{n-\operatorname{dim}(U)} \chi_{U} \mid U \text { an affine subspace of } \mathbb{F}_{2}^{n}\right\}
$$

Lemma 3.4.1 The lattice $M_{n}$ is the $n$-fold tensor product $M_{1} \underset{\mathbb{Z}[\sqrt{-2}]}{\otimes} \ldots \underset{\mathbb{Z}[\sqrt{-2}]}{\otimes} M_{1}$.

Proof: We use induction on $n$. Let $V_{n-1}=\left\langle e_{1}, \ldots, e_{n-1}\right\rangle$ and $V_{1}=\left\langle e_{n}\right\rangle$. One checks that $b_{x} \otimes b_{y}=b_{x+y}$ for all $x \in V_{n-1}, y \in V_{1}$.

Let $U$ be a $d$-dimensional affine subspace of $V_{n-1}$. For $y$ in $V_{1}$ it follows that $\sqrt{-2}^{n-1-d} \chi_{U} \otimes \sqrt{-2}_{y}=\sqrt{-2}^{n-d} \chi_{y+U} \in M_{n}$. Similarly, since $U+V_{1}$ has dimension $d+1$, we get $\sqrt{-2}^{n-1-d} \chi_{U} \otimes \chi_{V_{1}}=\sqrt{-2}^{n-(d+1)} \chi_{U+V_{1}} \in M_{n}$. Thus $M_{n-1} \otimes M_{1} \subseteq M_{n}$. Conversely, suppose $U$ is a $d$-dimensional affine subspace of $\mathbb{F}_{2}^{n}$. Write $U=x+y+U_{0}$ where $U_{0}$ is a subspace of $\mathbb{F}_{2}^{n}$ and $x \in V_{n-1}, y \in V_{1}$.

If $U_{0} \leq V_{n-1}$ then $\sqrt{-2}^{n-d} \chi_{U}=\sqrt{-2}^{n-1-d} \chi_{x+U} \otimes \sqrt{-2} b_{y} \in M_{n-1} \otimes M_{1}$. Otherwise $U_{n-1}:=U_{0} \cap V_{n-1}$ is a $(d-1)$-dimensional subspace and $U_{0}=U_{n-1} \cup\left(z+e_{n}+U_{n-1}\right)$ for some $z \in V_{n-1}$.

If $z \in U_{n-1}$, then $\sqrt{-2}^{n-d} \chi_{U}=\sqrt{-2}^{n-1-(d-1)} \chi_{x+U_{n-1}} \otimes \chi_{V_{1}}$ otherwise we have

$$
\begin{aligned}
\sqrt{-2}^{n-d} \chi_{U} & =\sqrt{-2}^{n-1-d} \chi_{x+U_{n-1}+\langle z\rangle} \otimes \sqrt{-2} b_{y} \\
& +\sqrt{-2}^{n-1-(d-1)} \chi_{x+z+U_{n-1}} \otimes \chi_{V_{1}} \\
& \left.-\sqrt{-2}^{n-1} \sqrt{-1-(d-1)}^{n-1)} \chi_{x+z+U_{n-1}} \otimes \sqrt{-2} b_{y}\right)
\end{aligned}
$$

This shows $M_{n} \subseteq M_{n-1} \otimes M_{1}$.

Lemma 3.4.2 The group $\mathcal{H}_{n}$ is conjugate to the Hermitian automorphism group $\operatorname{Aut}_{\mathbb{Q}(\sqrt{-2})}\left(M_{n}\right):=\operatorname{Stab}_{U_{2^{n}}(\mathbb{Q}(\sqrt{-2}))}\left(M_{n}\right)$ in $\mathrm{GL}_{2^{n}}(\mathbb{Q}(\sqrt{-2}))$.

Proof: For $n=3$ the result can be checked explicitly. So we may assume that $n \neq$ 3. Let $\left(v_{1}, \ldots, v_{2^{n}}\right)$ be a $\mathbb{Z}$-basis of $L_{n}^{\prime}$ such that $\left(2 v_{1}, \ldots, 2 v_{2^{n-1}}, v_{2^{n-1}+1}, \ldots, v_{2^{n}}\right)$ is a $\mathbb{Z}$-basis of $L_{n}$. Then $\left(\sqrt{-2} v_{1}, \ldots, \sqrt{-2} v_{2^{n}}, 2 v_{1}, \ldots, 2 v_{2^{n-1}}, v_{2^{n-1}+1}, \ldots, v_{2^{n}}\right)$ is a $\mathbb{Z}$-basis of $M_{n}=\sqrt{-2} L_{n}^{\prime} \oplus L_{n}$. In particular, the $\mathbb{Z}$-lattices $L_{n}$ and $\sqrt{-2} L_{n}^{\prime}$ are perpendicular with respect to the scalar product $(x, y) \mapsto \frac{1}{2} \operatorname{Tr}_{\mathbb{Q}(\sqrt{-2}) / \mathbb{Q}}\left(x \bar{y}^{\operatorname{tr}}\right)$. Thus the group $\operatorname{Aut}_{\mathbb{Q}(\sqrt{-2})}\left(M_{n}\right)$ is the subgroup of $\operatorname{Aut}\left(\sqrt{-2} L_{n}^{\prime} \perp L_{n}, I_{2^{n+1}}\right)$ which commutes with $\sqrt{-2}$. Since $n \neq 3$ is assumed, the automorphism groups of $L_{n}$ and $L_{n}^{\prime}$ equal $H_{n}$ [Wal62, Theorem 3.2]. Hence using appropriate bases, $\operatorname{Aut}_{\mathbb{Q}(\sqrt{-2})}\left(M_{n}\right)$ contains a subgroup $G_{n}$ of index at most two where

$$
G_{n}=\left\{\left(\begin{array}{cc}
g_{1} & 0 \\
0 & g_{2}
\end{array}\right) \mid g_{1}, g_{2} \in H_{n}\right\} \cap \operatorname{Aut}_{\mathbb{Q}(\sqrt{-2})}\left(M_{n}\right)
$$

Now $\sqrt{-2}$ interchanges $\sqrt{-2} L_{n}^{\prime}$ and $L_{n}$, i.e. it operates as a block matrix $\left(\begin{array}{cc}0 & w \\ -2 w^{-1} & 0\end{array}\right)$ for some $w \in \mathrm{GL}_{2^{n}}(\mathbb{Q})$. So $\left(\begin{array}{cc}g_{1} & 0 \\ 0 & g_{2}\end{array}\right) \in G_{n}$ if and only if $g_{2}=g_{1}^{w}$ and therefore $G_{n} \simeq H_{n}$. 
On the other hand, $\frac{1}{\sqrt{-2}} h_{n}$ acts on $M_{1} \otimes M_{n-1}=M_{n}$. So $\operatorname{Aut}_{\mathbb{Q}(\sqrt{-2})}\left(M_{n}\right)$ is isomorphic to $\mathcal{H}_{n}$. Both groups have conjugate Fitting subgroups and therefore their generalized Bravais groups must be conjugate as well. But then the whole groups must be conjugate, since $\mathcal{H}_{n}$ is the unique extension of $H_{n}$ in $\mathrm{GL}_{2^{n}}(\mathbb{Q}(\sqrt{-2}))$ by the extra automorphism induced by $h_{n}$.

Lemma 3.4.3 If $n \geq 2$, then $\sqrt{-2}\left[2_{+}^{1+2 n} \cdot\left(O_{2 n}^{+}(2): 2\right)\right]_{2^{n}}$ is the (up to conjugacy) unique s.i.m.f. subgroup of $\mathrm{Sp}_{2^{n+1}}(\mathbb{Q})$ with Fitting group $2_{+}^{1+2 n}$.

Proof: Since $\mathbb{Z}[\sqrt{-2}]$ is a PID, $M_{n}$ has a $\mathbb{Z}[\sqrt{-2}]$-basis. With respect to this basis, its automorphism group $G_{n}$ is a finite subgroup of $\mathrm{GL}_{2^{n}}(\mathbb{Z}[\sqrt{-2}])$. By explicit calculations in MAGMA one checks that for $n \in\{2,3\}$ the $\mathbb{Z}$-span $\left\langle G_{n}\right\rangle_{\mathbb{Z}}$ equals $\mathbb{Z}[\sqrt{-2}]^{2^{n} \times 2^{n}}$. For $n \geq 4$ it follows from $M_{n}=M_{n-2} \underset{\mathbb{Z}[\sqrt{-2}]}{\otimes} M_{2}$ that $G_{2} \otimes G_{n-2} \subset G_{n}$ (using appropriate bases). By induction, we get

$$
\mathbb{Z}[\sqrt{-2}]^{2^{n} \times 2^{n}}=\left\langle G_{n-2}\right\rangle_{\mathbb{Z}} \underset{\mathbb{Z}[\sqrt{-2}]}{\otimes}\left\langle G_{2}\right\rangle_{\mathbb{Z}} \subseteq\left\langle G_{n}\right\rangle_{\mathbb{Z}} \subseteq \mathbb{Z}[\sqrt{-2}]^{2^{n} \times 2^{n}}
$$

In particular, each $G_{n}$-invariant $\mathbb{Z}[\sqrt{-2}]$-lattice is a multiple of $M_{n}$, since $\mathbb{Z}[\sqrt{-2}]$ has class number 1 . Thus $G_{n}$ is a maximal finite subgroup of $\mathrm{GL}_{2^{n}}(\mathbb{Q}(\sqrt{-2}))$. But any finite symplectic supergroup of ${ }_{\sqrt{-2}}\left[2_{+}^{1+2 n} .\left(O_{2 n}^{+}(2): 2\right)\right]_{2^{n}}$ comes from a finite supergroup of $\mathcal{H}_{n} \simeq G_{n}<\mathrm{GL}_{2^{n}}(\mathbb{Q}(\sqrt{-2}))$. Thus $\sqrt{-2}_{-2}\left[2_{+}^{1+2 n} \cdot\left(O_{2 n}^{+}(2): 2\right)\right]_{2^{n}}$ is s.i.m.f.. The second statement follows from the construction of $\mathcal{H}_{n}$ and Remark 2.2.17.

\subsection{The group $p_{+}^{1+2 n}$}

In this section, let $p$ be an odd prime. We will describe a family of irreducible symplectic matrix groups in dimension $p^{n}(p-1)$ which will be maximal finite in the case that $p$ is a Fermat prime, i.e. $p-1$ is a power of two.

Let $T_{n}^{(p)} \simeq p_{+}^{1+2 n}$ be the $n$-fold tensor product of $T_{1}^{(p)}$ where $T_{1}^{(p)}$ is the subgroup of $\mathrm{GL}_{p}\left(\mathbb{Q}\left(\zeta_{p}\right)\right)$ generated by the diagonal matrix $\operatorname{Diag}\left(1, \zeta_{p}, \ldots, \zeta_{p}^{p-1}\right)$ and the permutation matrix corresponding to the $p$-cycle $(1, \ldots, p)$. Further let $H_{n}^{(p)}=$ $N_{U_{p^{n}}\left(\mathbb{Q}\left(\zeta_{p}\right)\right)}\left(T_{n}^{(p)}\right)$. By [Win72] $H_{n}^{(p)}$ is isomorphic to a subgroup of $C_{2} \times p_{+}^{1+2 n} \cdot \operatorname{Sp}_{2 n}(p)$, since the group of outer automorphisms which act trivially on the center of $T_{n}^{(p)}$ is isomorphic to $\operatorname{Sp}_{2 n}(p)$.

In Wal62, Section 4] Wall constructs a $(p-1) p^{n}$ dimensional rational lattice on which $C_{2} \times p_{+}^{1+2 n} \cdot \operatorname{Sp}_{2 n}(p)$ acts. Thus $H_{n}^{(p)}=\mathcal{B}^{o}\left(T_{n}^{(p)}\right) \simeq C_{2} \times p_{+}^{1+2 n} \cdot \operatorname{Sp}_{2 n}(p)$ (if we assume that $\left.T_{n}^{(p)} \unlhd \mathcal{B}^{o}\left(T_{n}^{(p)}\right)\right)$. Now $H_{n}^{(p)}$ gives rise to a finite subgroup of $\operatorname{Sp}_{p^{n}(p-1)}(\mathbb{Q})$ which will be denoted by $\sqrt{-p}\left[ \pm p_{+}^{1+2 n} \cdot \operatorname{Sp}_{2 n}(p)\right]_{p^{n}}$. 
Theorem 3.5.1 If $p$ is a Fermat prime, then $\sqrt{-p}\left[ \pm p_{+}^{1+2 n} \cdot \operatorname{Sp}_{2 n}(p)\right]_{p^{n}}$ is a s.i.m.f. subgroup of $\operatorname{Sp}_{p^{n}(p-1)}(\mathbb{Q})$.

Proof: The commuting algebra $C$ of $\sqrt{-p}\left[ \pm p_{+}^{1+2 n} \cdot \operatorname{Sp}_{2 n}(p)\right]_{p^{n}}$ is isomorphic to $\mathbb{Q}\left(\zeta_{p}\right)$. Since $p$ is a Fermat prime, $C$ has only one maximal subfield, which must be totally real. Thus any symplectic supergroup must come from a finite subgroup of $\mathrm{GL}_{p^{n}}\left(\mathbb{Q}\left(\zeta_{p}\right)\right)$. But $H_{n}^{(p)}$ is maximal finite in $\mathrm{GL}_{p^{n}}\left(\mathbb{Q}\left(\zeta_{p}\right)\right)$ according to [NRS01, Theorem 7.3]. 


\section{Chapter 4}

\section{The classification}

In this final chapter, we classify all s.i.m.f. subgroups of $\mathrm{GL}_{2 n}(\mathbb{Q})$ for $1 \leq n \leq 11$.

The first section handles the generic case $n>3$ prime. The subsequent sections each deal with one particular dimension $2 n$. The s.i.m.f. subgroups $G<\mathrm{GL}_{2 n}(\mathbb{Q})$ are listed in tables as follows:

The s.i.m.f. groups are grouped together by their commuting algebras. Groups having the same commuting algebras are sorted by group order (descending) and minimal determinant $d$ (ascending). The invariant $d$ is explained below.

The first column contains a number for the group for easy referencing. The primitive groups are numbered consecutively. For the imprimitive groups we use triples $[i, j, k]$ with $j k=2 n$ which stands for the wreath product of the $i$-th s.p.i.m.f. matrix group in dimension $j$ with $S_{k}$.

The second column contains the name of $G$ using the conventions from Definition 2.4.2. The next two columns list the group order $|G|$ and the number of isomorphism classes of $G$-invariant lattices. These two numbers and the endomorphism ring (which can be read off the second column) are invariants of the conjugacy class of $G$. But usually this does not identify the class of $G$ uniquely.

Thus the fifth column (labeled $L_{\text {min }}$ ) contains some more invariants: Let

$$
\begin{aligned}
S & :=\left\{(L, F) \in \mathcal{Z}(G) \times \mathcal{F}_{>0}(G) \mid F \text { integral on } L\right\} \quad \text { and } \\
d & :=\min \{\operatorname{det}(L, F) \mid(L, F) \in S\} .
\end{aligned}
$$

Then $\{(L, F) \in S \mid \operatorname{det}(L, F)=d\}$ partitions into several isometry classes. If $\left(L_{1}, F_{1}\right), \ldots,\left(L_{s}, F_{s}\right)$ represent these classes, then the third column lists the distinct triples $\left[d, \min \left(L_{i}, F_{i}\right),\left|\mathrm{SV}\left(L_{i}, F_{i}\right)\right|\right]$ for $(1 \leq i \leq s)$. Here $\min (L, F)$ and $\operatorname{SV}(L, F)$ denote the minimum and the set of shortest vectors of $(L, F)$ respectively.

It turns out that in almost all cases, these invariants determine the conjugacy class of $G$ uniquely. (In fact, the only exceptions are $\left(\sqrt{5}, \infty_{\infty}\left[\mathrm{SL}_{2}(5)\right]_{1} \circ C_{5}\right) \otimes A_{2}$ and $\zeta_{10}\left[C_{10}\right]_{1} \underset{\sqrt{5}^{\prime}}{\otimes_{3}, 3}\left[\mathrm{SL}_{2}(9)\right]_{2}<\mathrm{Sp}_{16}(\mathbb{Q})$. But they can easily be distinguised by their Fitting subgroups.)

Finally the last column lists the r.i.m.f. supergroups of $G$ (up to conjugacy). 


\subsection{Dimension $2 \mathrm{p}$}

Let $G$ be a s.p.i.m.f. matrix group of degree $2 p$ for some prime $p>3$.

Lemma 4.1.1 The Fitting subgroup $F(G)$ is cyclic of order 2,4 or 6 .

Proof: By Corollary 2.1.16 $O_{l}(G)=1$ for all primes $\ell$ with $\ell-1 \nmid 2 p$. Suppose first $q:=2 p+1$ is prime and $q|| G \mid$. Then $q \geq 11$ and $q \equiv_{4}-1$. So Theorem 3.1.1 shows that $G \simeq \sqrt{-q}\left[ \pm \mathrm{L}_{2}(q)\right]_{p}$ and thus $F(G) \simeq C_{2}$.

So we may now assume that $F(G)=O_{2}(G) O_{3}(G)$. In particular $G$ cannot be cyclic since otherwise $G$ is either reducible or $O_{q}(G) \neq 1$. Thus $G$ embeds irreducibly into $\mathrm{GL}_{p}(K)$ for some imaginary quadratic number field $K$. So the result follows from Table 2.5.2, since $D_{8}$ does not have such an embedding.

Corollary 4.1.2 The group $G$ is not soluble. Moreover, $K:=\operatorname{End}(\bar{G})$ is an imaginary quadratic number field such that $\triangle_{K}(E(G))<\mathrm{GL}_{p}(K)$ is absolutely irreducible.

Thus $E(G)$ is either reducible with $\mathbb{Q}^{2 \times 2}$ as commuting algebra or it is irreducible and its commuting algebra is an imaginary quadratic number field. In both cases, $G$ can easily be recovered from $F^{*}(G)$ (cf. Sections 2.2.3 and 2.2.4).

\subsection{Dimension 2}

Theorem 4.2.1 The s.i.m.f. subgroups $G$ of $\mathrm{Sp}_{2}(\mathbb{Q})$ are

\begin{tabular}{|c|c|c|c|c|c|}
\hline$\#$ & $G$ & $|G|$ & $|\mathcal{Z}(G)|$ & $L_{\min }$ & r.i.m.f. supergroups \\
\hline \hline 1 & ${ }_{i}\left[C_{4}\right]_{1}$ & $2^{2}$ & 1 & {$[1,1,4]$} & $B_{2}$ \\
\hline \hline 2 & ${ }_{\sqrt{-3}}\left[C_{6}\right]_{1}$ & $2 \cdot 3$ & 1 & {$[3,2,6]$} & $A_{2}$ \\
\hline
\end{tabular}

Proof: Every finite subgroup of $\mathrm{Sp}_{2}(\mathbb{Q})$ is cyclic since it admits a faithful representation of degree 1 over some imaginary quadratic number field. 


\subsection{Dimension 4}

Theorem 4.3.1 The s.i.m.f. subgroups $G$ of $\mathrm{Sp}_{4}(\mathbb{Q})$ are

\begin{tabular}{|c|c|c|c|c|c|}
\hline$\#$ & $G$ & $|G|$ & $|\mathcal{Z}(G)|$ & $L_{\text {min }}$ & r.i.m.f. supergroups \\
\hline \hline 1 & ${ }_{i}\left[\left(D_{8} \otimes C_{4}\right) . S_{3}\right]_{2}$ & $2^{5} \cdot 3$ & 1 & {$\left[2^{2}, 2,24\right]$} & $F_{4}$ \\
\hline 2 & ${ }_{i}\left[C_{4}\right]_{1} \otimes A_{2}$ & $2^{3} \cdot 3$ & 2 & {$\left[3^{2}, 2,12\right]$} & $A_{2}^{2}$ \\
\hline \hline 3 & $\sqrt{-2}\left[\mathrm{GL}_{2}(3)\right]_{2}$ & $2^{4} \cdot 3$ & 1 & {$\left[2^{2}, 2,24\right]$} & $F_{4}$ \\
\hline \hline 4 & $\infty, 2\left[\mathrm{SL}_{2}(3)\right]_{1} \circ C_{3}$ & $2^{3} \cdot 3^{2}$ & 2 & {$\left[2^{2}, 2,24\right]$} & $F_{4}$ \\
\hline$[2,2,2]$ & ${ }_{\sqrt{-3}}\left[C_{6}\right]_{1}^{2}$ & $2^{3} \cdot 3^{2}$ & 1 & {$\left[3^{2}, 2,12\right]$} & $A_{2}^{2}$ \\
\hline \hline 5 & $\zeta_{10}\left[C_{10}\right]_{1}$ & $2 \cdot 5$ & 1 & {$[5,2,20]$} & $A_{4}$ \\
\hline
\end{tabular}

Proof: It follows from explicit calculations, that the above table is correct and yields s.i.m.f. groups. The r.i.m.f. supergroups are easily constructed, since all these groups except $\zeta_{10}\left[C_{10}\right]_{1}$ are uniform. Further $\zeta_{10}\left[C_{10}\right]_{1}$ can only be contained in $\operatorname{Aut}\left(A_{4}\right)$ by comparison of orders. The imprimitive s.i.m.f. matrix groups can only be conjugate to ${ }_{\sqrt{-3}}\left[C_{6}\right]_{1}^{2}$ or ${ }_{i}\left[C_{4}\right]_{1}^{2}$. The first group if s.i.m.f. by Lemma 2.1.21. The group $H:={ }_{i}\left[C_{4}\right]_{1}^{2}$ has $\mathbb{Q}(i)$ as commuting algebra. Further it fixes two lattices $L_{1}, L_{2}$. One checks that one of the groups $\operatorname{Aut}\left(L_{j}, \mathcal{F}(H)\right)(j=1,2)$ is conjugate to ${ }_{i}\left[\left(D_{8} \otimes C_{4}\right) . S_{3}\right]_{2}$. Thus $H$ is not maximal finite.

So it remains to prove that each s.p.i.m.f. subgroup of $\operatorname{Sp}_{4}(\mathbb{Q})$ is conjugate to one of the groups from above. This is done in Section 4.3.2.

\subsubsection{Irreducible cyclic subgroups}

Before we prove the completeness, we classify all finite subgroups $G<\operatorname{Sp}_{4}(\mathbb{Q})$ that contain an irreducible cyclic subgroup $U$. Then $| \pm U| \in\{8,10,12\}$.

Lemma 4.3.2 If $|U|=8$ then $G$ is conjugate to ${ }_{\sqrt{-2}}\left[\mathrm{GL}_{2}(3)\right]_{2}$ or ${ }_{i}\left[\left(D_{8} \otimes C_{4}\right) . S_{3}\right]_{2}$.

Proof: It follows from Minkowski's bound that $\Pi(G) \subseteq\{2,3,5\}$. The commuting algebra of $U$ is isomorphic to $\mathbb{Q}\left(\zeta_{8}\right)$ and has class number 1 . So $U$ fixes only one lattice $L$. One finds some $F \in \mathcal{F}_{>0}(U)$ that is integral on $L$ such that $\operatorname{det}(L, F)=1$. By Table 2.5.3 $G$ must be conjugate to $\operatorname{Aut}_{\mathbb{Q}(\sqrt{-d})}\left(L, \mathfrak{p}_{2}^{a} F\right)$ for some $a \in\{0,1\}$ and $d \in\{1,2\}$. This leaves the candidates:

\begin{tabular}{|c|c|c|}
\hline & $\mathbb{Q}(i)$ & $\mathbb{Q}(\sqrt{-2})$ \\
\hline \hline$F$ & ${ }_{i}\left[\left(D_{8} \otimes C_{4}\right) \cdot S_{3}\right]_{2}$ & $\subsetneq \sqrt{-2}\left[\mathrm{GL}_{2}(3)\right]_{2}$ \\
\hline $\mathfrak{p}_{2} F$ & ${ }_{i}\left[\left(D_{8} \otimes C_{4}\right) \cdot S_{3}\right]_{2}$ & $\sqrt{-2}\left[\mathrm{GL}_{2}(3)\right]_{2}$ \\
\hline
\end{tabular}

Lemma 4.3.3 If $\pm U$ is of order 10 then $G \simeq \zeta_{10}\left[C_{10}\right]_{1}$. 
Proof: The commuting algebra $\operatorname{End}(\bar{U}) \simeq \mathbb{Q}\left(\zeta_{10}\right)$ has only one proper subfield, which is $\mathbb{Q}(\sqrt{5})$. So $\operatorname{End}(\bar{U})=\operatorname{End}(\bar{G})$. Further $U$ fixes up to isomorphism only one lattice $L$. Let $F \in \mathcal{F}_{>0}(U)$, then $\operatorname{Aut}_{\mathbb{Q}\left(\zeta_{10}\right)}(L, F)= \pm U$ shows that $\pm U$ is s.i.m.f..

Lemma 4.3.4 If $U$ is of order 12 then $G$ is conjugate to ${ }_{i}\left[\left(D_{8} \otimes C_{4}\right) . S_{3}\right]_{2},{ }_{i}\left[C_{4}\right]_{1} \otimes A_{2}$, $\infty, 2\left[\mathrm{SL}_{2}(3)\right]_{1} \circ C_{3}$ or $\sqrt{-3}\left[C_{6}\right]_{1}^{2}$.

Proof: Again by Minkowski's bound $\Pi(G) \subseteq\{2,3,5\}$. The commuting algebra of $U$ is isomorphic to $\mathbb{Q}\left(\zeta_{12}\right)$ and has class number 1 . So $U$ fixes only one lattice $L$. Further, one finds some $F \in \mathcal{F}_{>0}(U)$ that is integral on $L$ such that $\operatorname{det}(L, F)=4$. By Table 2.5.3. $G$ must be conjugate to $\operatorname{Aut}_{\mathbb{Q}(\sqrt{-d})}\left(L, \mathfrak{p}_{2}^{-a} \mathfrak{p}_{3}^{a} F\right)$ for some $a \in\{0,1\}$ and $d \in\{1,3\}$. This leaves the four candidates:

\begin{tabular}{|c|c|c|}
\hline & $\mathbb{Q}(i)$ & $\mathbb{Q}(\sqrt{-3})$ \\
\hline \hline$F$ & ${ }_{i}\left[\left(D_{8} \otimes C_{4}\right) . S_{3}\right]_{2}$ & $\infty, 2\left[\mathrm{SL}_{2}(3)\right]_{1} \circ C_{3}$ \\
\hline $\mathfrak{p}_{2}^{-1} \mathfrak{p}_{3} F$ & ${ }_{i}\left[C_{4}\right]_{1} \otimes A_{2}$ & $\sqrt{-3}\left[C_{6}\right]_{1}^{2}$ \\
\hline
\end{tabular}

So the result follows.

\subsubsection{Proof of Theorem 4.3.1}

Let $G<\operatorname{Sp}_{4}(\mathbb{Q})$ be s.p.i.m.f.. Then $E(G)=1$ according to Table 2.5.1. Thus $F(G)$ is selfcentralizing.

By Corollary 2.1.16 we know that $O_{p}(G)=1$ for all $p \geq 7$. The case $O_{5}(G) \neq 1$ is handled in Lemma 4.3.3. The remaining cases are handled in the two subsequent lemmas.

Lemma 4.3.5 If $O_{3}(G) \neq 1$ then $G$ is conjugate to ${ }_{i}\left[C_{4}\right]_{1} \otimes A_{2}$ or $\infty_{\infty, 2}\left[\mathrm{SL}_{2}(3)\right]_{1} \circ C_{3}$.

Proof: By Table 2.5.2 we get $O_{3}(G)=C_{3}$ and $O_{2}(G)$ is one of $C_{2}, C_{4}, D_{8}$ or $Q_{8}$. If $\mathrm{O}_{2}(G)=C_{2}$ then $\left[G: C_{6}\right] \leq\left|\operatorname{Out}\left(C_{6}\right)\right|=2$ contradicts Lemma 2.2.1. In all other cases $G$ contains an irreducible cyclic subgroup of order 12 , so the result follows from Lemma 4.3.4.

Lemma 4.3.6 If $F(G)$ is a 2-group, then $G$ is conjugate to ${ }_{i}\left[\left(D_{8} \otimes C_{4}\right) . S_{3}\right]_{2}$ or $\sqrt{-2}\left[\mathrm{GL}_{2}(3)\right]_{2}$.

Proof: We already know that $\mathcal{B}^{\circ}\left(D_{8} \otimes C_{4}\right)={ }_{i}\left[\left(D_{8} \otimes C_{4}\right) . S_{3}\right]_{2}$ is maximal finite. Assume now that $O_{2}(G)$ is cyclic or isomorphic to $D_{8}$, then $G=O_{2}(G)$ since $E(G)=1$ and $\operatorname{Out}(F(G)))$ is a 2-group. Thus $G=O_{2}(G)$ is either reducible or not maximal. If $O_{2}(G) \simeq Q D_{16}$ then $G$ contains an irreducible cyclic subgroup of order 8 . This contradicts Lemma 4.3.2. So there remains the case that $O_{2}(G) \simeq Q_{8}$. Then $B:=\mathcal{B}^{o}\left(O_{2}(G)\right) \simeq{ }_{\infty, 2}\left[\mathrm{SL}_{2}(3)\right]_{1}$ and $[G: B]=2$ since $B$ is not maximal. By Remark 2.2.17 there is only one such extension, which is $\sqrt{-2}\left[\mathrm{GL}_{2}(3)\right]_{2}$. 


\subsection{Dimension 6}

Theorem 4.4.1 The s.i.m.f. subgroups $G$ of $\operatorname{Sp}_{6}(\mathbb{Q})$ are

\begin{tabular}{|c|c|c|c|c|c|}
\hline$\#$ & $G$ & $|G|$ & $|\mathcal{Z}(G)|$ & $L_{\text {min }}$ & r.i.m.f. supergroups \\
\hline \hline$[2,1,3]$ & ${ }_{i}\left[C_{4}\right]_{1}^{3}$ & $2^{7} \cdot 3$ & 3 & {$[1,1,12]$} & $B_{6}$ \\
\hline \hline 1 & $\sqrt{-3}\left[ \pm 3_{+}^{1+2}: \mathrm{SL}_{2}(3)\right]_{3}$ & $2^{4} \cdot 3^{4}$ & 2 & {$[3,2,72]$} & $E_{6}$ \\
\hline$[2,2,3]$ & $\sqrt{-3}\left[C_{6}\right]_{1}^{3}$ & $2^{4} \cdot 3^{4}$ & 1 & {$\left[3^{3}, 2,18\right]$} & $A_{2}^{3}$ \\
\hline \hline 2 & $\sqrt{-7}\left[ \pm \mathrm{L}_{2}(7)\right]_{3}$ & $2^{4} \cdot 3 \cdot 7$ & 1 & {$\left[7^{3}, 4,42\right]$} & $A_{6}^{(2)}$ \\
\hline
\end{tabular}

We need two lemmas for the proof. The first lemma shows that Theorem 3.1.1 also holds for $p=7$.

Lemma 4.4.2 Let $G<\operatorname{Sp}_{6}(\mathbb{Q})$ be s.i.m.f. such that $|G|$ is divisible by 7. Then $G$ is conjugate to $\sqrt{-7}\left[ \pm \mathrm{L}_{2}(7)\right]_{3}$.

Proof: As in the proof of Theorem 3.1.1, it follows that $G$ is symplectic primitive and contains an irreducible subgroup $U$ isomorphic to $C_{7}$. By Minkowski's bound we get $\Pi(|G|) \subseteq\{2,3,5,7\}$. The group $U$ fixes only one lattice $L$ since $\operatorname{End}(\bar{U}) \simeq \mathbb{Q}\left(\zeta_{14}\right)$ has class number 1. Further there exists some $F \in \mathcal{F}_{>0}(U)$ that is integral on $L$ with $\operatorname{det}(L, F)=7$. Table 2.5.3 shows that $G$ is conjugate to $\operatorname{Aut}_{\mathbb{Q}(\sqrt{-7})}\left(L, \mathfrak{p}_{7}^{k} F\right)$ for some $k \in\{0,1\}$. So the result is easily verified.

Lemma 4.4.3 Let $G<\operatorname{Sp}_{6}(\mathbb{Q})$ be s.p.i.m.f. such that $\left|O_{3}(G)\right|>3$ or $G$ contains an irreducible subgroup $U \simeq C_{18}$. Then $O_{3}(G) \simeq 3_{+}^{1+2}$ and $G \simeq \sqrt{-3}\left[ \pm 3_{+}^{1+2}: \mathrm{SL}_{2}(3)\right]_{3}$.

Proof: If $\left|O_{3}(G)\right|>3$ then, by Table 2.5.2, $O_{3}(G)$ is either cyclic of order 9 or isomorphic to $3_{+}^{1+2}$. In the latter case $\mathcal{B}^{o}\left(O_{3}(G)\right) \simeq \sqrt{-3}\left[ \pm 3_{+}^{1+2}: \mathrm{SL}_{2}(3)\right]_{3}$ and this group is s.i.m.f. by Theorem 3.5.1. So we may assume that $G$ contains an irreducible subgroup $U \simeq C_{18}$. The commuting algebra of $U$ is isomorphic to $\mathbb{Q}\left(\zeta_{18}\right)$ and has class number 1 . Thus $U$ fixes only one lattice $L$. One finds some $F \in \mathcal{F}_{>0}(U)$ that is integral on $L$ such that $\operatorname{det}(L, F)=3$. Table 2.5 .3 shows that $G$ is conjugate to $\operatorname{Aut}_{\sqrt{-3}}\left(L, \mathfrak{p}_{3}^{k} F\right)$ for some $k \in\{0,1\}$. These two automorphism groups are conjugate to $\sqrt{-3}\left[ \pm 3_{+}^{1+2}: \mathrm{SL}_{2}(3)\right]_{3}$ and $\sqrt{-3}\left[C_{6}\right]_{1}^{3}$ respectively.

Proof (of Theorem 4.4.1): One checks explicitely that the table given in Theorem 4.4.1 is correct. (Note that the r.i.m.f. supergroups are easily constructed sind all s.i.m.f. groups are uniform.) The group $\sqrt{-3}\left[C_{6}\right]_{1}^{3}$ is s.i.m.f. by Lemma 2.1.21. The group ${ }_{i}\left[C_{4}\right]_{1}^{3}$ fixes three lattices and has $\mathbb{Q}(i)$ as commuting algebra. So one checks that it is maximal. Thus it remains to show that each s.p.i.m.f. group $G<\mathrm{Sp}_{6}(\mathbb{Q})$ is conjugate to $\sqrt{-3}\left[ \pm 3_{+}^{1+2}: \mathrm{SL}_{2}(3)\right]_{3}$ or ${ }_{\sqrt{-7}}\left[ \pm \mathrm{L}_{2}(7)\right]_{3}$. By Table 2.5.2 and the preceding lemmas we may assume that $O_{3}(G) \leq C_{3}$ and $O_{p}(G)=1$ for all primes $p>3$. If $E(G)=1$ then $F(G) \in\left\{ \pm I_{6}, C_{4}, \pm C_{3}, D_{8}\right\}$. In any case $[G: F(G)] \leq 2$, which contradicts Lemma 2.2.1. Hence $E(G) \neq 1$ and from Table 2.5.1 it follows that $E(G) \simeq \mathrm{L}_{2}(7)$. But then $G \simeq \sqrt{-7}\left[ \pm \mathrm{L}_{2}(7)\right]_{3}$ by the above. 


\subsection{Dimension 8}

Theorem 4.5.1 The s.i.m.f. subgroups $G$ of $\operatorname{Sp}_{8}(\mathbb{Q})$ are:

\begin{tabular}{|c|c|c|c|c|c|}
\hline$\#$ & $G$ & $|G|$ & $|\mathcal{Z}(G)|$ & $L_{\min }$ & $\begin{array}{c}\text { r.i.m.f. } \\
\text { supergroups }\end{array}$ \\
\hline $\bar{~} 1$ & ${ }_{\bar{i} i[}\left[\left(2_{+}^{1+4} \otimes C_{4}\right) \cdot S_{6}\right]_{4}$ & $2^{10} \cdot 3^{2} \cdot 5$ & 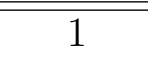 & $\overline{[1,2,240]}$ & $\overline{\bar{E}}$ \\
\hline$[4,1,2]$ & ${ }_{i}\left[\left(D_{8} \otimes C_{4}\right) \cdot S_{3}\right]_{2}^{2}$ & $2^{11} \cdot 3^{2}$ & 1 & {$\left[2^{4}, 2,48\right]$} & $F_{4}^{2}$ \\
\hline$[2,1,4]$ & ${ }_{i}\left[C_{4}\right]_{1}^{4}$ & $2^{11} \cdot 3$ & 3 & {$[1,1,16]$} & $B_{8}$ \\
\hline$[4,2,2]$ & $\left({ }_{i}\left[C_{4}\right]_{1} \otimes A_{2}\right)^{2}$ & $2^{7} \cdot 3^{2}$ & 2 & {$\left[3^{4}, 2,24\right]$} & $A_{2}^{4}$ \\
\hline 2 & ${ }_{i}\left[\left(D_{8} \otimes C_{4}\right) \cdot S_{3}\right]_{2} \otimes A_{2}$ & $2^{6} \cdot 3^{2}$ & 2 & {$\left[2^{4} \cdot 3^{4}, 4,72\right]$} & $A_{2} \otimes F_{4}$ \\
\hline 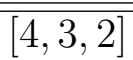 & $\frac{\sqrt{-2}\left[\mathrm{GL}_{2}(3)\right]_{2}^{2}}{}$ & $22^{9} \cdot 3^{2}$ & 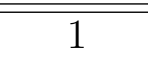 & {$\left[2^{4}, 2,48\right]$} & $\overline{F_{4}^{2}}$ \\
\hline 3 & $\sqrt{-2}\left[\infty, 2\left[2_{-}^{1+4} \cdot \text { Alt }_{5}\right]_{2}: 2\right]_{4}$ & $2^{8} \cdot 3 \cdot 5$ & 1 & {$[1,2,240]$} & $E_{8}$ \\
\hline 4 & $\begin{array}{c}\sqrt{-2}\left[2_{+}^{1+4} \cdot\left(O_{4}^{+}(2): 2\right)\right]_{4} \\
=\sqrt{-2}\left[F_{4}: 2\right]_{4}\end{array}$ & $2^{8} \cdot 3^{2}$ & 1 & {$\left[2^{4}, 2,48\right]$} & $F_{4}^{2}$ \\
\hline 5 & $\sqrt{-3}\left[\operatorname{Sp}_{4}(3) \circ C_{3}\right]_{4}$ & $2^{7} \cdot 3^{5} \cdot 5$ & 1 & {$[1,2,240]$} & $E_{8}$ \\
\hline$[2,2,4]$ & $\sqrt{-3}\left[C_{6}\right]_{1}^{4}$ & $2^{7} \cdot 3^{5}$ & 1 & {$\left[3^{4}, 2,24\right]$} & $A_{2}^{4}$ \\
\hline$[4,4,2]$ & $\left(\infty, 2\left[\mathrm{SL}_{2}(3)\right]_{1} \circ C_{3}\right)^{2}$ & $2^{7} \cdot 3^{4}$ & 2 & {$\left[2^{4}, 2,48\right]$} & $F_{4}^{2}$ \\
\hline 6 & $\sqrt{-3}\left[C_{6}\right]_{1} \otimes F_{4}$ & $2^{7} \cdot 3^{3}$ & 2 & {$\left[2^{4} \cdot 3^{4}, 4,72\right]$} & $A_{2} \otimes F_{4}$ \\
\hline 7 & $\infty, 5\left[\mathrm{SL}_{2}(5): 2\right]_{2} \circ C_{3}$ & $2^{4} \cdot 3^{2} \cdot 5$ & 2 & {$\left[5^{4}, 4,120\right]$} & $S$ \\
\hline 8 & $\sqrt{-3}\left[C_{6}\right]_{1} \otimes A_{4}$ & $2^{4} \cdot 3^{2} \cdot 5$ & 2 & {$\left[3^{4} \cdot 5^{2}, 4,60\right]$} & $A_{2} \otimes A_{4}$ \\
\hline 9 & $\sqrt{-5}\left[\sqrt{5}, \infty\left[\mathrm{SL}_{2}(5)\right]_{1}^{2-} \square C_{4}\right]_{4}$ & $2^{5} \cdot 3 \cdot 5$ & 2 & {$[1,2,240]$} & $E_{8}$ \\
\hline 10 & $\sqrt{-5}\left[\sqrt{5}, \infty\left[\mathrm{SL}_{2}(5)\right]_{1}{ }^{2+} C_{4}\right]_{4}$ & $2^{5} \cdot 3 \cdot 5$ & 2 & {$\left[5^{4}, 4,120\right]$} & $S$ \\
\hline 11 & $\sqrt{-5}\left[C_{20}: C_{4}\right]_{4}$ & $2^{4} \cdot 5$ & 4 & {$\left[5^{2}, 2,40\right]$} & $A_{4}^{2}$ \\
\hline 12 & $\sqrt{-6}\left[\sqrt{2}, \infty\left[\tilde{S}_{4}\right]_{1}^{2} \square C_{3}\right]_{4}$ & $2^{5} \cdot 3^{2}$ & 2 & {$[1,2,240]$} & $E_{8}$ \\
\hline 13 & $\sqrt{-6}\left[\sqrt{2}, \infty\left[\tilde{S}_{4}\right]_{1}{ }^{2+} C_{3}\right]_{4}$ & $2^{5} \cdot 3^{2}$ & 2 & {$\left[2^{4}, 2,48\right]$} & $F_{4}^{2}$ \\
\hline 14 & $\sqrt{-6}\left[D_{16} \stackrel{2}{\otimes}_{\sqrt{-3}}\left[C_{6}\right]_{1}\right]_{4}$ & $2^{5} \cdot 3$ & 4 & {$\left[3^{4}, 2,24\right]$} & $A_{2}^{4}, A_{2} \otimes F_{4}$ \\
\hline 15 & $\sqrt{-7}\left[2 . \mathrm{Alt}_{7}\right]_{4}$ & $2^{4} \cdot 3^{2} \cdot 5 \cdot 7$ & 1 & {$[1,2,240]$} & $\overline{E_{8}}$ \\
\hline 16 & $\sqrt{-15}\left[\sqrt{5}, \infty\left[\mathrm{SL}_{2}(5)\right]_{1}^{2} \square C_{3}\right]_{4}$ & $2^{4} \cdot 3^{2} \cdot 5$ & 2 & {$[1,2,240]$} & $E_{8}$ \\
\hline 17 & $\sqrt{-15}\left[\sqrt{5}, \infty\left[\mathrm{SL}_{2}(5)\right]_{1}^{2+} C_{3}\right]_{4}$ & $2^{4} \cdot 3^{2} \cdot 5$ & 2 & {$\left[5^{4}, 4,120\right]$} & $S$ \\
\hline 18 & $\sqrt{-15}\left[C_{30}: C_{4}\right]_{4}$ & $2^{3} \cdot 3 \cdot 5$ & 4 & {$\left[3^{4} \cdot 5^{2}, 4,60\right]$} & $A_{2} \otimes A_{4}$ \\
\hline 19 & $\sqrt{5}, \infty\left[\mathrm{SL}_{2}(5)\right]_{1} \circ C_{5}$ & $2^{3} \cdot 3 \cdot 5^{2}$ & 1 & {$[1,2,240]$} & $E_{8}, S$ \\
\hline$[4,5,2]$ & $\zeta_{10}\left[C_{10}\right]_{1}^{2}$ & $2^{3} \cdot 5^{2}$ & 1 & {$\left[5^{2}, 2,40\right]$} & $A_{4}^{2}$ \\
\hline 20 & $\zeta_{10}\left[C_{10}\right]_{1} \otimes A_{2}$ & $2^{2} \cdot 3 \cdot 5$ & 2 & {$\left[3^{4} \cdot 5^{2}, 4,60\right]$} & $A_{2} \otimes A_{4}$ \\
\hline 21 & $\zeta_{16-\zeta_{16}^{-1}}\left[Q D_{32}\right]_{2}$ & $2^{5}$ & 2 & {$[1,1,16]$} & $B_{8}, F_{4}^{2}$ \\
\hline
\end{tabular}

where $S$ denotes the r.i.m.f. matrix group $\left[\left(\mathrm{SL}_{2}(5) \stackrel{2}{\square} \mathrm{SL}_{2}(5)\right): 2\right]_{8}$.

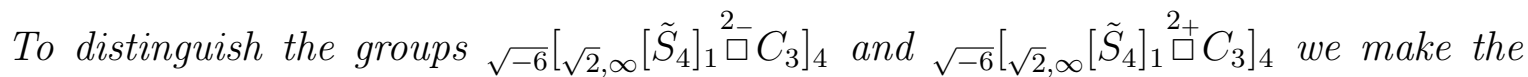
following convention. Both groups are generated by $N:={ }_{\sqrt{2}, \infty}\left[\tilde{S}_{4}\right]_{1} \circ C_{3}$ and some $\alpha \in$ $G$ with $\alpha^{2} \in N$ that centralizes $\mathcal{B}^{\circ}\left(O_{2}(G)\right) \simeq{ }_{\infty, 2}\left[\mathrm{SL}_{2}(3)\right]_{1}$ and induces the nontrivial 
outer automorphisms on $\tilde{S}_{4}$ and $C_{3}$. Up to isomorphism there are two choices for $\alpha^{2}$. The name $\sqrt{-6}\left[\sqrt{2}, \infty\left[\tilde{S}_{4}\right]_{1} \stackrel{2_{+}}{\square} C_{3}\right]_{4}$ stands for $\alpha^{2}=I_{8}$ and we write $\sqrt{-6}\left[\sqrt{2}, \infty\left[\tilde{S}_{4}\right]_{1}^{2-} C_{3}\right]_{4}$ if $\alpha^{2}=-I_{8}$.

The proof of the theorem is given in Section 4.5.2.

\subsubsection{Irreducible cyclic subgroups}

If $G<\operatorname{Sp}_{8}(\mathbb{Q})$ is s.i.m.f. and contains an irreducible cyclic subgroup $U$ then $| \pm U| \in$ $\{16,20,24,30\}$. In this section, we construct all such groups $G$.

Lemma 4.5.2 Let $G<\operatorname{Sp}_{8}(\mathbb{Q})$ be s.i.m.f. with an irreducible subgroup $U \simeq C_{16}$. Suppose that $\Pi(|G|) \subseteq\{2,3,5\}$ or that there exists some $(L, F) \in \mathcal{Z}(G) \times \mathcal{F}_{>0}(G)$ such that $F$ is integral on $L$ and $\Pi(\operatorname{det}(L, F)) \subseteq\{2,3,5\}$. Then $G$ is conjugate to one of the following groups:

$$
\zeta_{16-\zeta_{16}^{-1}}\left[Q D_{32}\right]_{2},{ }_{i}\left[C_{4}\right]_{1}^{4}, \sqrt{-2}\left[\mathrm{GL}_{2}(3)\right]_{2}^{2},{ }_{i}\left[\left(D_{8} \otimes C_{4}\right) \cdot S_{3}\right]_{2}^{2} .
$$

Proof: The commuting algebra of $U$ is isomorphic to $\mathbb{Q}\left(\zeta_{16}\right)$ and has class number 1 . Thus $U$ fixes only one lattice $L$. One finds some $F \in \mathcal{F}_{>0}(U)$ such that $F$ is integral on $L$ and $\operatorname{det}(L, F)=1$. By Table 2.5.3, we have to consider the $\operatorname{groups} \operatorname{Aut}_{K}\left(L, \mathfrak{p}_{2}^{k} F\right)$ for some $0 \leq k \leq 2$ and $K \in\left\{\mathbb{Q}(i), \mathbb{Q}(\sqrt{-2}), \mathbb{Q}\left(\zeta_{16}-\zeta_{16}^{-1}\right)\right\}$.

\begin{tabular}{|c|c|c|c|}
\hline form & $\mathbb{Q}(i)$ & $\mathbb{Q}(\sqrt{-2})$ & $\mathbb{Q}\left(\zeta_{16}-\zeta_{16}^{-1}\right)$ \\
\hline \hline$F$ & ${ }_{i}\left[C_{4}\right]_{1}^{4}$ & $\lessgtr \sqrt{-2}\left[\mathrm{GL}_{2}(3)\right]_{2}^{2}$ & $\zeta_{16}-\zeta_{16}^{-1}\left[Q D_{32}\right]_{2}$ \\
\hline $\mathfrak{p}_{2} F$ & ${ }_{i}\left[C_{4}\right]_{1}^{4}$ & $\lesseqgtr \sqrt{-2}\left[\mathrm{GL}_{2}(3)\right]_{2}^{2}$ & $\zeta_{16}-\zeta_{16}^{-1}\left[Q D_{32}\right]_{2}$ \\
\hline $\mathfrak{p}_{2}^{2} F$ & ${ }_{i}\left[\left(D_{8} \otimes C_{4}\right) \cdot S_{3}\right]_{2}^{2}$ & $\sqrt{-2}\left[\mathrm{GL}_{2}(3)\right]_{2}^{2}$ & $\zeta_{16-\zeta_{16}^{-1}}\left[Q D_{32}\right]_{2}$ \\
\hline
\end{tabular}

So the result follows.

Lemma 4.5.3 Let $G<\operatorname{Sp}_{8}(\mathbb{Q})$ be s.i.m.f. with an irreducible subgroup $U \simeq C_{20}$. Suppose that $\Pi(|G|) \subseteq\{2,3,5\}$ or that there exists some $(L, F) \in \mathcal{Z}(G) \times \mathcal{F}_{>0}(G)$ such that $F$ is integral on $L$ and $\Pi(\operatorname{det}(L, F)) \subseteq\{2,3,5\}$. Then $G$ is conjugate to $\zeta_{10}\left[C_{10}\right]_{1}^{2}$, $\sqrt{-5}\left[C_{20}: 4\right]_{4}, \sqrt{5}, \infty\left[\mathrm{SL}_{2}(5)\right]_{1} \circ C_{5}, \sqrt{-5}\left[\sqrt{5}, \infty\left[\mathrm{SL}_{2}(5)\right]_{1} \stackrel{2_{+}}{\square} C_{4}\right]_{4}, \sqrt{-5}\left[\sqrt{5}, \infty\left[\mathrm{SL}_{2}(5)\right]_{1}^{2-} C_{4}\right]_{4}$ or ${ }_{i}\left[\left(2_{+}^{1+4} \otimes C_{4}\right) \cdot S_{6}\right]_{4}$.

Proof: The commuting algebra of $U$ is isomorphic to $\mathbb{Q}\left(\zeta_{20}\right)$ and has class number 1 . Thus $U$ fixes only one lattice $L$. One finds some $F \in \mathcal{F}_{>0}(U)$ such that $F$ is integral on $L$ and $\operatorname{det}(L, F)=1$. By Table 2.5.3, we have to consider the $\operatorname{groups} \operatorname{Aut}_{K}\left(L, \mathfrak{p}_{5}^{k} F\right)$ for some $0 \leq k \leq 2$ and $K \in\left\{\mathbb{Q}(i), \mathbb{Q}(\sqrt{-5}), \mathbb{Q}\left(\zeta_{10}\right)\right\}$.

\begin{tabular}{|c|c|c|c|}
\hline form & $\mathbb{Q}(i)$ & $\mathbb{Q}(\sqrt{-5})$ & $\mathbb{Q}\left(\zeta_{10}\right)$ \\
\hline \hline$F$ & ${ }_{i}\left[\left(2_{+}^{1+4} \otimes C_{4}\right) \cdot S_{6}\right]_{4}$ & $\sqrt{-5}\left[\sqrt{5}, \infty\left[\mathrm{SL}_{2}(5)\right]_{1}{ }^{2-} C_{4}\right]_{4}$ & $\sqrt{5, \infty}\left[\mathrm{SL}_{2}(5)\right]_{1} \circ C_{5}$ \\
\hline $\mathfrak{p}_{5} F$ & $\Varangle_{i}\left[\left(2_{+}^{1+4} \otimes C_{4}\right) \cdot S_{6}\right]_{4}$ & $\sqrt{-5}\left[C_{20}: 4\right]_{4}$ & $\zeta_{10}\left[C_{10}\right]_{1}^{2}$ \\
\hline $\mathfrak{p}_{5}^{2} F$ & $\Varangle_{i}\left[\left(2_{+}^{1+4} \otimes C_{4}\right) \cdot S_{6}\right]_{4}$ & $\sqrt{-5}\left[\sqrt{5}, \infty\left[\mathrm{SL}_{2}(5)\right]_{1}{ }^{2+} C_{4}\right]_{4}$ & ${ }_{\sqrt{5}, \infty}\left[\mathrm{SL}_{2}(5)\right]_{1} \circ C_{5}$ \\
\hline
\end{tabular}

This proves the claim. 
Lemma 4.5.4 Let $G<\operatorname{Sp}_{8}(\mathbb{Q})$ be s.i.m.f. with an irreducible subgroup $U \simeq C_{24}$. Suppose that $\Pi(|G|) \subseteq\{2,3,5\}$ or that there exists some $(L, F) \in \mathcal{Z}(G) \times \mathcal{F}_{>0}(G)$ such that $F$ is integral on $L$ and $\Pi(\operatorname{det}(L, F)) \subseteq\{2,3,5\}$. Then $G$ is conjugate to one of:

$$
\begin{aligned}
& \sqrt{-6}\left[\sqrt{2}, \infty\left[\tilde{S}_{4}\right]_{1} \stackrel{2_{+}}{\square} C_{3}\right]_{4}, \sqrt{-6}\left[\sqrt{2}, \infty\left[\tilde{S}_{4}\right]_{1}^{2-} C_{3}\right]_{4},{ }_{i}\left[\left(\left(D_{8} \otimes C_{4}\right) . S_{3}\right)\right]_{2} \otimes A_{2}, \\
& \sqrt{-2}\left[\infty, 2\left[2_{-}^{1+4} \cdot \mathrm{Alt}_{5}\right]_{2}: 2\right]_{4},{ }_{i}\left[\left(2_{+}^{1+4} \otimes C_{4}\right) \cdot S_{6}\right]_{4}, \sqrt{-3}\left[\operatorname{Sp}_{4}(3) \circ C_{3}\right]_{4},\left({ }_{i}\left[C_{4}\right]_{1} \otimes A_{2}\right)^{2} \text {, } \\
& \sqrt{-3}\left[C_{6}\right]_{1}^{4},\left(\infty, 2\left[\mathrm{SL}_{2}(3)\right]_{1} \circ C_{3}\right)^{2},{ }_{i}\left[\left(\left(D_{8} \otimes C_{4}\right) . S_{3}\right)\right]_{2}^{2}, \sqrt{-3}\left[C_{6}\right]_{1} \otimes F_{4} \text {, } \\
& \infty, 5\left[\mathrm{SL}_{2}(5): 2\right]_{2} \circ C_{3}, \sqrt{-6}\left[D_{16} \stackrel{2}{\otimes}_{\sqrt{-3}}\left[C_{6}\right]_{1}\right]_{4}, \sqrt{-2}\left[F_{4}: 2\right]_{4} \text {. }
\end{aligned}
$$

Proof: The commuting algebra $\operatorname{End}(\bar{U}) \simeq \mathbb{Q}\left(\zeta_{30}\right)$ has class number 1 , so $U$ fixes up to isomorphism one lattice $L$. One finds some $F \in \mathcal{F}_{>0}(U)$ such that $F$ is integral on $L$ and $\operatorname{det}(L, F)=1$. By Table 2.5.3 we have to consider the groups $\operatorname{Aut}_{\mathbb{Q}(\sqrt{-d})}\left(L, \mathfrak{p}_{2}^{2 a} \mathfrak{p}_{3}^{b} \mathfrak{p}_{5}^{c} \mathfrak{p}_{5}^{\prime c^{\prime}} F\right)$ for $a, b, c, c^{\prime} \in\{0,1\}$ and $d \in\{1,2,3,6\}$.

\begin{tabular}{|c|c|c|}
\hline form & $\mathbb{Q}(i)$ & $\mathbb{Q}(\sqrt{-2})$ \\
\hline$\overline{F F}$ & $\bar{i} \bar{i}\left[\left(2_{+}^{1+4} \otimes C_{4}\right) \cdot S_{6}\right]_{4}$ & $\bar{i}_{i}\left[\infty, 2\left[2_{-}^{1+4} \cdot \mathrm{Alt}_{5}\right]_{2}: 2\right]_{4}$ \\
\hline $\mathfrak{p}_{2}^{2} F$ & ${ }_{i}\left[\left(D_{8} \otimes C_{4}\right) \cdot S_{3}\right]_{2}^{2}$ & $\sqrt{-2}\left[F_{4}: 2\right]_{4}$ \\
\hline $\mathfrak{p}_{3} F$ & $\left({ }_{i}\left[C_{4}\right]_{1} \otimes A_{2}\right)^{2}$ & $\succ_{i}\left[\infty, 2\left[2_{-}^{1+4} \cdot \mathrm{Alt}_{5}\right]_{2}: 2\right]_{4}$ \\
\hline $\mathfrak{p}_{2}^{2} \mathfrak{p}_{3} F$ & ${ }_{i}\left[\left(D_{8} \otimes C_{4}\right) \cdot S_{3}\right]_{2} \otimes A_{2}$ & $\lesseqgtr \sqrt{-2}\left[F_{4}: 2\right]_{4}$ \\
\hline $\mathfrak{p}_{5} F$ & $\succ_{i}\left[\left(2_{+}^{1+4} \otimes C_{4}\right) \cdot S_{6}\right]_{4}$ & 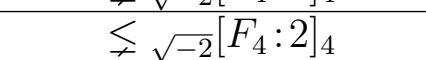 \\
\hline $\mathfrak{p}_{2}^{2} \mathfrak{p}_{5} F$ & $\lesseqgtr\left({ }_{i}\left[C_{4}\right]_{1} \otimes A_{2}\right)^{2}$ & $\left.\lesseqgtr_{i[\infty, 2}\left[2_{-}^{1+4} \cdot \mathrm{Alt}_{5}\right]_{2}: 2\right]_{4}$ \\
\hline $\mathfrak{p}_{3} \mathfrak{p}_{5} F$ & ${ }_{i}\left[\left(2_{+}^{1+4} \otimes C_{4}\right) \cdot S_{6}\right]_{4}$ & $\underbrace{}_{\sqrt{-2}}\left[F_{4}: 2\right]_{4}$ \\
\hline $\mathfrak{p}_{2}^{2} \mathfrak{p}_{3} \mathfrak{p}_{5} F$ & $\lesseqgtr\left({ }_{i}\left[C_{4}\right]_{1} \otimes A_{2}\right)^{2}$ & $\lesseqgtr_{i}\left[\infty, 2\left[2_{-}^{1+4} \cdot \mathrm{Alt}_{5}\right]_{2}: 2\right]_{4}$ \\
\hline form & $\mathbb{Q}(\sqrt{-3})$ & $\mathbb{Q}(\sqrt{-6})$ \\
\hline$F$ & $\sqrt{-3}\left[\operatorname{Sp}_{4}(3) \circ C_{3}\right]_{4}$ & $\sqrt{-6}\left[\sqrt{2}, \infty\left[\tilde{S}_{4}\right]_{1}^{2} \square C_{3}\right]_{4}$ \\
\hline $\mathfrak{p}_{2}^{2} F$ & $\left(\infty, 2\left[\mathrm{SL}_{2}(3)\right]_{1} \circ C_{3}\right)^{2}$ & $\sqrt{-6}\left[\sqrt{2}, \infty\left[\tilde{S}_{4}\right]_{1}{ }^{2+} \square C_{3}\right]_{4}$ \\
\hline $\mathfrak{p}_{3} F$ & $\sqrt{-3}\left[C_{6}\right]_{1}^{4}$ & $\sqrt{-6}\left[D_{16} \stackrel{2}{\otimes}_{\sqrt{-3}}\left[C_{6}\right]_{1}\right]_{4}$ \\
\hline $\mathfrak{p}_{2}^{2} \mathfrak{p}_{3} F$ & $\sqrt{-3}\left[C_{6}\right]_{1} \otimes F_{4}$ & $\sqrt{-6}\left[D_{16} \stackrel{2}{\otimes}_{\sqrt{-3}}\left[C_{6}\right]_{1}\right]_{4}$ \\
\hline $\mathfrak{p}_{5} F$ & $\infty, 5\left[\mathrm{SL}_{2}(5): 2\right]_{2} \circ C_{3}$ & $\lessgtr \sqrt{-6}\left[\sqrt{2}, \infty\left[\tilde{S}_{4}\right]_{1}^{2-} C_{3}\right]_{4}$ \\
\hline $\mathfrak{p}_{2}^{2} \mathfrak{p}_{5} F$ & $\underbrace{}_{-3}\left[\operatorname{Sp}_{4}(3) \circ C_{3}\right]_{4}$ & $\lesseqgtr \sqrt{-6}\left[D_{16} \otimes_{\sqrt{-3}}\left[C_{6}\right]_{1}\right]_{4}$ \\
\hline $\mathfrak{p}_{3} \mathfrak{p}_{5} F$ & $\lessgtr \infty, 5\left[\mathrm{SL}_{2}(5): 2\right]_{2} \circ C_{3}$ & $\lesseqgtr \sqrt{-6}\left[\sqrt{2}, \infty\left[\tilde{S}_{4}\right]_{1}{ }^{2-} C_{3}\right]_{4}$ \\
\hline $\mathfrak{p}_{2}^{2} \mathfrak{p}_{3} \mathfrak{p}_{5} F$ & $\lesseqgtr \sqrt{-3}\left[\operatorname{Sp}_{4}(3) \circ C_{3}\right]_{4}$ & $\lessgtr \sqrt{-6}\left[D_{16} \stackrel{2}{\otimes}_{\sqrt{-3}}\left[C_{6}\right]_{1}\right]_{4}$ \\
\hline
\end{tabular}

Since there exists some $x \in N_{\mathrm{GL}_{8}(\mathbb{Q})}(U) \cap \mathrm{GL}(L)$ such that $\mathfrak{p}_{5}^{x}=\mathfrak{p}_{5}^{\prime}$ (and necessarily $\left.\mathfrak{p}_{2}^{x}=\mathfrak{p}_{2}, \mathfrak{p}_{3}^{x}=\mathfrak{p}_{3}\right)$ we may assume that $c^{\prime} \leq c$. Since $\mathfrak{p}_{5} \mathfrak{p}_{5}^{\prime}=5 \mathbb{Z}\left[\theta_{24}\right]$ we may even assume that $c^{\prime}=0$. So we have 32 possibilities:

This proves the claim. 
Lemma 4.5.5 Let $G<\operatorname{Sp}_{8}(\mathbb{Q})$ be s.i.m.f. with an irreducible subgroup $U \simeq C_{30}$. Suppose that $\Pi(|G|) \subseteq\{2,3,5\}$ or that there exists some $(L, F) \in \mathcal{Z}(G) \times \mathcal{F}_{>0}(G)$ such that $F$ is integral on $L$ and $\Pi(\operatorname{det}(L, F)) \subseteq\{2,3,5\}$. Then $G$ is conjugate to one of the following groups:

$$
\begin{gathered}
\sqrt{-3}\left[\mathrm{Sp}_{4}(3) \circ C_{3}\right]_{4}, \zeta_{10}\left[C_{10}\right]_{1} \otimes A_{2}, \sqrt{-15}\left[C_{30}: 4\right]_{4}, \sqrt{-3}\left[C_{6}\right]_{1} \otimes A_{4}, \infty, 5\left[\mathrm{SL}_{2}(5): 2\right]_{2} \circ C_{3}, \\
\sqrt{-15}\left[\sqrt{5}, \infty\left[\mathrm{SL}_{2}(5)\right]_{1}{ }^{2+} C_{3}\right]_{4}, \sqrt{-15}\left[\sqrt{5}, \infty\left[\mathrm{SL}_{2}(5)\right]_{1}^{2-} C_{3}\right]_{4}, \sqrt{5}, \infty\left[\mathrm{SL}_{2}(5)\right]_{1} \circ C_{5} .
\end{gathered}
$$

Proof: The commuting algebra $\operatorname{End}(\bar{U}) \simeq \mathbb{Q}\left(\zeta_{30}\right)$ has class number 1 , so $U$ fixes up to isomorphism one lattice $L$. Further there exists some $F \in \mathcal{F}_{>0}(U)$ such that $F$ is integral on $L$ and $\operatorname{det}(L, F)=1$. By Table 2.5.3 we have to consider the groups $\operatorname{Aut}_{K}\left(L, \mathfrak{p}_{3}^{a} \mathfrak{p}_{5}^{b} F\right)$ where $a \in\{0,1\}, b \in\{0,1,2\}$ with $a \equiv_{2} b$ and $K \in\left\{\mathbb{Q}(\sqrt{-3}), \mathbb{Q}(\sqrt{-15}), \mathbb{Q}\left(\zeta_{10}\right)\right\}$. So we have the following 9 automorphism groups to check:

\begin{tabular}{|c|c|c|c|}
\hline form & $\mathbb{Q}(\sqrt{-3})$ & $\mathbb{Q}(\sqrt{-15})$ & $\mathbb{Q}\left(\zeta_{10}\right)$ \\
\hline \hline$F$ & $\sqrt{-3}\left[\mathrm{Sp}_{4}(3) \circ C_{3}\right]_{4}$ & $\sqrt{-15}\left[\sqrt{5}, \infty\left[\mathrm{SL}_{2}(5)\right]_{1}^{2} \square C_{3}\right]_{4}$ & $\sqrt{5, \infty}\left[\mathrm{SL}_{2}(5)\right]_{1} \circ C_{5}$ \\
\hline $\mathfrak{p}_{3} \mathfrak{p}_{5} F$ & $\sqrt{-3}\left[C_{6}\right]_{1} \otimes A_{4}$ & $\sqrt{-15}\left[C_{30}: 4\right]_{4}$ & $\zeta_{10}\left[C_{10}\right]_{1} \otimes A_{2}$ \\
\hline $\mathfrak{p}_{5}^{2} F$ & $\infty, 5\left[\mathrm{SL}_{2}(5): 2\right]_{2} \circ C_{3}$ & $\sqrt{-15}\left[\sqrt{5}, \infty\left[\mathrm{SL}_{2}(5)\right]_{1}{ }^{2} C_{3}\right]_{4}$ & $\sqrt{5, \infty}\left[\mathrm{SL}_{2}(5)\right]_{1} \circ C_{5}$ \\
\hline
\end{tabular}

These groups are all maximal finite.

\subsubsection{Proof of Theorem 4.5.1}

We have to prove the completeness of the list given in Theorem 4.5.1. The candidates for the maximal finite symplectic imprimitive groups come from the classification of the s.p.i.m.f. subgroups of $\operatorname{Sp}_{2}(\mathbb{Q})$ and $\operatorname{Sp}_{4}(\mathbb{Q})$. By Lemma 2.1.21, we only have to check the group ${ }_{i}\left[C_{4}\right]_{1}^{4}$. It fixes up to isomorphism three lattices and has $\mathbb{Q}(i)$ as commuting algebra. One verifies that it is s.i.m.f..

So it remains to prove the completeness for the s.p.i.m.f. subgroups of $\operatorname{Sp}_{8}(\mathbb{Q})$. Thus, for the remainder of this section let $G<\operatorname{Sp}_{8}(\mathbb{Q})$ be s.p.i.m.f..

First we handle the case that $G$ contains a quasisimple normal subgroup $N$. By

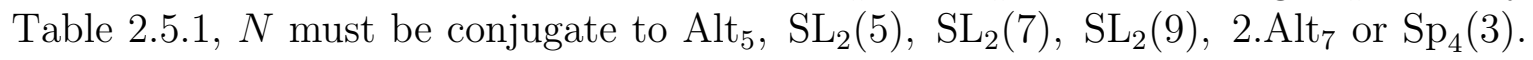
These cases are handled in the next two lemmas.

Lemma 4.5.6 Let $N$ be a normal subgroup of $G$.

(a) If $N$ is conjugate to $\mathrm{Alt}_{5}$ then $G \simeq \sqrt{-3}\left[C_{6}\right]_{1} \otimes A_{4}$.

(b) If $N$ is conjugate to $2 . \mathrm{Alt}_{7}$, then $G=N$.

(c) If $N$ is conjugate to $\operatorname{Sp}_{4}(3)$, then $G=\mathcal{B}^{\circ}(N) \simeq \sqrt{-3}\left[\operatorname{Sp}_{4}(3) \circ C_{3}\right]_{4}$. 
(d) $N$ is not conjugate to $\mathrm{SL}_{2}(7)$.

(e) $N$ is not conjugate to $\mathrm{SL}_{2}(9)$.

Proof:

(a) Let $H:=\mathcal{B}^{\circ}(N) \simeq \pm S_{5} \simeq \operatorname{Aut}\left(A_{4}\right)$. So $H$ cannot be self centralizing since $\operatorname{Out}(H)$ is trivial and $\operatorname{End}(\bar{H}) \simeq \mathbb{Q}^{2 \times 2}$. Thus $G$ must contain a subgroup conjugate to $C_{k} \otimes A_{4}$ with $k \in\{6,4\}$. These groups have $\mathbb{Q}(\sqrt{-3})$ and $\mathbb{Q}(i)$ as commuting algebras and they fix 2 and 4 lattices respectively. One easily checks that $\sqrt{-3}\left[C_{6}\right]_{1} \otimes A_{4}$ is s.i.m.f. whereas $C_{4} \otimes A_{4}$ is properly contained in ${ }_{i}\left[\left(2_{+}^{1+4} \otimes C_{4}\right) \cdot S_{6}\right]_{4}$.

(b) Up to isomorphism, $N$ fixes only one lattice $L$ and it has $\mathbb{Q}(\sqrt{-7})$ as commuting algebra. So the claim is easily verified.

(c) Again, $\mathcal{B}^{\circ}(N) \simeq{ }_{\sqrt{-3}}\left[\operatorname{Sp}_{4}(3) \circ C_{3}\right]_{4}$ fixes only one lattice $L$ and has $\mathbb{Q}(\sqrt{-3})$ as commuting algebra. The result follows as above.

(d) $N$ fixes 5 lattices and has $\mathbb{Q}(\sqrt{-7})$ as commuting algebra. Let $F \in \mathcal{F}_{>0}(N)$. One checks that $\operatorname{Aut}_{\mathbb{Q}(\sqrt{-7})}(L, F)$ is either conjugate to $N$ or $\sqrt{-7}\left[2 . \mathrm{Alt}_{7}\right]_{4}$ for all $L \in \mathcal{Z}(N)$.

(e) The commuting algebra of $N$ is isomorphic to $\mathcal{Q}_{\infty, 3}$ and $\operatorname{Out}(N) \simeq C_{2} \times C_{2}$ but only one class of outer automorphisms can be realized in $\mathrm{GL}_{8}(\mathbb{Q})$. Using Remark 2.2.17, one finds that $G$ contains a subgroup conjugate to $N \circ C_{4}, N \circ C_{3}$ or N.2. The first group fixes 2 lattices and is only contained in ${ }_{i}\left[\left(2_{+}^{1+4} \otimes C_{4}\right) \cdot S_{6}\right]_{4}$ the other two groups fix only one lattice and they are only contained in $\sqrt{-3}\left[\mathrm{Sp}_{4}(3) \circ C_{3}\right]_{4}$. So the result follows.

Lemma 4.5.7 If $G$ contains a normal subgroup $N$ conjugate to $\sqrt{5}, \infty\left[\mathrm{SL}_{2}(5)\right]_{1}$, then $G$ is conjugate to one of

$$
\begin{aligned}
& \sqrt{-15}\left[\sqrt{5}, \infty\left[\mathrm{SL}_{2}(5)\right]_{1} \stackrel{2_{-}}{\square} C_{3}\right]_{4}, \sqrt{-15}\left[\sqrt{5}, \infty\left[\mathrm{SL}_{2}(5)\right]_{1} \stackrel{2_{+}}{\square} C_{3}\right]_{4}, \quad \infty, 5\left[\mathrm{SL}_{2}(5): 2\right]_{2} \circ C_{3}, \\
& \sqrt{-5}\left[\sqrt{5}, \infty\left[\mathrm{SL}_{2}(5)\right]_{1} \stackrel{2_{-}}{\square} C_{4}\right]_{4}, \sqrt{-5}\left[\sqrt{5}, \infty\left[\mathrm{SL}_{2}(5)\right]_{1}{ }^{+} C_{4}\right]_{4} \text { or } \sqrt{5, \infty}\left[\mathrm{SL}_{2}(5)\right]_{1} \circ C_{5} .
\end{aligned}
$$

Proof: Let $Q:=\operatorname{End}(\bar{N}) \simeq \mathcal{Q}_{\sqrt{5}, \infty}$ and let $K \simeq \mathbb{Q}(\sqrt{5})$ be its center. Further denote by $\mathfrak{M}$ a maximal $\mathbb{Z}_{K}$-order of $Q$. If $C_{G}(N) \subset N$, then $[G: N]=|\operatorname{Out}(N)|=2$. The outer automorphism of $N$ does not centralize $K$. So $\operatorname{End}(\bar{G})<Q$ is a totally definite quaternion algebra with center $\mathbb{Q}$ ramified at a subset of $\Pi(|G|)=\{2,3,5\}$. Hence it contains nontrivial torsion units which contradicts the assumption $C_{G}(N) \subset N$.

Thus there exists some $g \in C_{G}(N) \backslash N$. We may assume that $g$ is contained in the torsion group $\mathfrak{M}^{*, 1} \simeq \mathrm{SL}_{2}(5)$, since all maximal $\mathbb{Z}_{K}$-orders of $Q$ are conjugate. This leaves three cases and in any case $\Pi(|G|)=\{2,3,5\}$. 
- $U:=N \circ C_{3} \leq G$. Then $K$ is the maximal totally real subfield of $\operatorname{End}(\bar{U}) \simeq \mathbb{Q}(\sqrt{-3}, \sqrt{5})$. Further $U$ fixes only one lattice $L$ and there exists some $F \in \mathcal{F}_{>0}(U)$ which is integral on $L$ with $\operatorname{det}(L, F)=1$. By the 2-parameter argument (see Table 2.5.4), $G$ must either fix $(L, F)$ or $\left(L, \mathfrak{p}_{5} F\right)$. The minimal totally complex subfields of $K$ are isomorphic to $\mathbb{Q}(\sqrt{-3})$ and $\mathbb{Q}(\sqrt{-15})$, this gives rise to four candidates:

\begin{tabular}{|c|c|c|}
\hline form & $\mathbb{Q}(\sqrt{-3})$ & $\mathbb{Q}(\sqrt{-15})$ \\
\hline \hline$F$ & $\sqrt{-3}\left[\mathrm{Sp}_{4}(3) \circ C_{3}\right]_{4}$ & $\sqrt{-15}\left[\sqrt{5, \infty}\left[\mathrm{SL}_{2}(5)\right]_{1}^{2-} C_{3}\right]_{4}$ \\
\hline $\mathfrak{p}_{5} F$ & $\infty, 5\left[\mathrm{SL}_{2}(5): 2\right]_{2} \circ C_{3}$ & $\sqrt{-15}\left[\sqrt{5, \infty}\left[\mathrm{SL}_{2}(5)\right]_{1}^{2} \square C_{3}\right]_{4}$ \\
\hline
\end{tabular}

- $U:=N \circ C_{4} \leq G$. Then $\operatorname{End}(\bar{U}) \simeq \mathbb{Q}(i, \sqrt{5})$. As above, $U$ fixes only one lattice $L$ and there exists some $F \in \mathcal{F}_{>0}(U)$ which is integral on $L$ and $\operatorname{det}(L, F)=1$. Again we have four candidates

\begin{tabular}{|c|c|c|}
\hline form & $\mathbb{Q}(i)$ & $\mathbb{Q}(\sqrt{-5})$ \\
\hline \hline$F$ & ${ }_{i}\left[\left(2_{+}^{1+4} \otimes C_{4}\right) \cdot S_{6}\right]_{4}$ & $\sqrt{-5}\left[\sqrt{5}, \infty\left[\mathrm{SL}_{2}(5)\right]_{1}{ }^{2} \square C_{4}\right]_{4}$ \\
\hline $\mathfrak{p}_{5} F$ & $\leq_{i}\left[\left(2_{+}^{1+4} \otimes C_{4}\right) \cdot S_{6}\right]_{4}$ & $\sqrt{-5}\left[\sqrt{5}, \infty\left[\mathrm{SL}_{2}(5)\right]_{1}{ }^{2} \square C_{4}\right]_{4}$ \\
\hline
\end{tabular}

- $U:=N \circ C_{5} \leq G$. Then $\operatorname{End}(\bar{U}) \simeq \mathbb{Q}\left(\zeta_{10}\right)$ is minimal totally complex. Further $U$ fixes only one lattice $L$. One checks that $U$ is already s.i.m.f..

After omitting the groups which do not contain a normal subgroup conjugate to $N$, one gets the claimed result.

We now turn to a case to case discussion of the various Fitting subgroups.

Lemma 4.5.8 If $O_{5}(G) \neq 1$ then $G$ is conjugate to one of the following groups:

$$
\zeta_{10}\left[C_{10}\right]_{1} \otimes A_{2}, \sqrt{-15}\left[C_{30}: C_{4}\right]_{4}, \sqrt{-4}\left[C_{20}: C_{4}\right]_{4} \text { or } \sqrt{5}, \infty\left[\mathrm{SL}_{2}(5)\right]_{1} \circ C_{5} .
$$

Proof: Table 2.5.2 shows $O_{5}(G) \simeq C_{5}$. If $O_{3}(G) \simeq C_{3}$ then $G$ contains an irreducible cyclic normal subgroup of order 30. Since $\left|\operatorname{Aut}\left(C_{30}\right)\right|=8$, it follows from Lemma 4.5.5 that $G \simeq \zeta_{10}\left[C_{10}\right]_{1} \otimes A_{2}$ or $\sqrt{-15}\left[C_{30}: C_{4}\right]_{4}$.

Suppose now $O_{3}(G)=1$. By Table 2.5.2, $O_{2}(G)$ must be conjugate to one of $C_{2}, C_{4}, D_{8}$ or $Q_{8}$ (note that $\mathbb{Q}\left(\zeta_{10}\right)$ splits $\mathcal{Q}_{\infty, 2}$ ). In the latter three cases, $G$ contains an irreducible cyclic subgroup of order 20 and $\Pi(|G|) \subseteq\{2,3,5\}$. Hence $G \simeq \sqrt{-4}\left[C_{20}: C_{4}\right]_{4}$ by Lemma 4.5.3.

If $O_{2}(G) \simeq C_{2}$, then $C_{G}(F(G))$ embeds into $\mathrm{GL}_{2}\left(\mathbb{Q}\left(\zeta_{10}\right)\right)$. If $E(G) \neq 1$, then by Table 2.5.1, $E(G)$ is conjugate to ${ }_{\sqrt{5}, \infty}\left[\mathrm{SL}_{2}(5)\right]_{1}$. In this case, Lemma 4.5 .7 shows that $G \simeq{ }_{\sqrt{5}, \infty}\left[\mathrm{SL}_{2}(5)\right]_{1} \circ C_{5}$. It remains the case that $F^{*}(G) \simeq C_{10}$. Then $G / F^{*}(G) \simeq \operatorname{Aut}\left(C_{10}\right) \simeq C_{4}$ by Lemma 2.2.1. There are two such extensions. The group $C_{10}: C_{4}$ is reducible, whereas $C_{10} \cdot C_{4}$ has $\mathcal{Q}_{\infty, 5}$ as commuting algebra. This group cannot be maximal finite since $\mathcal{Q}_{\infty, 5}$ contains nontrivial torsion units. 
$O_{3}(G) \neq 1$ and $O_{5}(G)=1$

We now suppose that $O_{5}(G)=1$ and $O_{3}(G) \neq 1$. By Table 2.5.2 we have $O_{3}(G) \simeq C_{3}$ and $O_{2}(G) \in\left\{C_{2}, C_{4}, C_{8}, D_{8}, Q_{8}, 2_{+}^{1+4}, 2_{-}^{1+4}, D_{8} \otimes C_{4}, D_{16}, Q D_{16}, Q_{16}\right\}$.

These cases are handled in the following lemmas.

\section{Lemma 4.5.9}

(a) If $O_{2}(G) \simeq D_{16}$ then $G \simeq{ }_{\sqrt{-6}}\left[D_{16} \stackrel{2}{\otimes}_{\sqrt{-3}}\left[C_{6}\right]_{1}\right]_{4}$.

(b) $\mathrm{O}_{2}(G)$ is not conjugate to $C_{8}, Q D_{16}$ or $Q_{16}$.

Proof: In all these cases $G$ contains an irreducible cyclic subgroup of order 24 and $\Pi(|G|)=\{2,3,5\}$. So the result follows from Lemma 4.5.4.

Lemma 4.5.10 If $O_{2}(G) \simeq 2_{+}^{1+4}$, then $G \simeq{ }_{\sqrt{-3}}\left[C_{6}\right]_{1} \otimes F_{4}$.

Proof: $\mathcal{B}^{\circ}(F(G))$ is conjugate to $\sqrt{-3}\left[C_{6}\right]_{1} \otimes F_{4}$ and fixes only one lattice. Its commuting algebra is isomorphic to $\mathbb{Q}(\sqrt{-3})$. Hence the claim is easily verified.

Lemma 4.5.11 $O_{2}(G) \not 2_{-}^{1+4}$.

Proof: Suppose $O_{2}(G) \simeq 2_{-}^{1+4}$. Then $G$ contains the normal subgroup $N:=\mathcal{B}^{\circ}(F(G))$ conjugate to $\infty_{\infty, 2}\left[2_{-}^{1+4} \text {. Alt }\right]_{2} \circ C_{3}$. The group $N$ fixes 2 lattices and has $\mathbb{Q}(\sqrt{-3})$ as commuting algebra. One easily checks that it is only contained in $\sqrt{-3}\left[\operatorname{Sp}_{4}(3) \circ C_{3}\right]_{4}$ (which has the wrong Fitting subgroup).

Lemma 4.5.12 If $O_{2}(G) \simeq D_{8} \otimes C_{4}$, then $G \simeq{ }_{i}\left[\left(D_{8} \otimes C_{4}\right) . S_{3}\right]_{2} \otimes A_{2}$.

Proof: The subgroup $N:=\mathcal{B}^{\circ}(F(G)) \simeq{ }_{i}\left[\left(D_{8} \otimes C_{4}\right) . S_{3}\right]_{2} \otimes C_{3}$ of $G$ contains an irreducible cyclic subgroup of order 24 and $\Pi(|G|)=\{2,3\}$. So the result follows from Lemma 4.5.4.

Lemma 4.5.13 $O_{2}(G) \not D_{8}$ and $O_{2}(G) \not C_{4}$.

Proof: Suppose $O_{2}(G)$ is one of these groups. In either case $G$ contains an irreducible normal subgroup $N \leq F(G)$ which is isomorphic to $C_{12}$. Then $C:=C_{G}(N)$ embeds into $\mathrm{GL}_{2}\left(\mathbb{Q}\left(\zeta_{12}\right)\right)$ and contains $N$. Thus $E(G)=1$ and $G / F(G) \leq \operatorname{Out}(F(G)) \simeq C_{2} \times$ $C_{2}$. If $O_{3}(G) \nsubseteq Z(G)$ then there exists some $g \in G$ such that $g$ induces the nontrivial outer automorphism on $O_{3}(G)$ and $g^{2} \in O_{2}(G)$. By maximality we get $g \in O_{2}(G)$ which contradicts $O_{2}(G) \subset C_{G}\left(O_{3}(G)\right)$. So $G / F(G) \leq C_{2}$. But this contradicts Lemma 2.2.1. 
Lemma 4.5.14 If $\mathrm{O}_{2}(G) \simeq C_{2}$ then $G$ is conjugate to $\sqrt{-3}\left[C_{6}\right]_{1} \otimes A_{4}, \sqrt{-3}\left[\operatorname{Sp}_{4}(3) \circ C_{3}\right]_{4}$, $\infty, 5\left[\mathrm{SL}_{2}(5): 2\right]_{2} \circ C_{3}, \sqrt{-15}\left[\sqrt{5}, \infty\left[\mathrm{SL}_{2}(5)\right]_{1}^{\left.\stackrel{2}{\square} C_{3}\right]_{4} \text { or } \sqrt{-15}[\sqrt{5}, \infty}\left[\mathrm{SL}_{2}(5)\right]_{1} \stackrel{2_{+}}{\square} C_{3}\right]_{4}$.

Proof: If $E(G)=1$, then $G / F(G) \leq \operatorname{Out}\left(C_{6}\right) \simeq C_{2}$ contradicts Lemma 2.2.1. So $E(G) \neq 1$. But all these cases have already been handled in Lemmas 4.5.6 and 4.5.7.

Lemma 4.5.15 If $O_{2}(G) \simeq Q_{8}$ then $G$ is either conjugate to $\sqrt{-6}\left[\sqrt{2}, \infty\left[\tilde{S}_{4}\right]_{1} \stackrel{2_{+}}{\square} C_{3}\right]_{4}$ or $\sqrt{-6}\left[\sqrt{2}, \infty\left[\tilde{S}_{4}\right]_{1} \stackrel{2-}{\square} C_{3}\right]_{4}$.

Proof: Let $N:=\mathcal{B}^{\circ}(F(G)) \simeq_{\infty, 2}\left[\mathrm{SL}_{2}(3)\right]_{1} \circ C_{3}$. Then $C:=C_{G}(N)$ embeds into $\mathrm{GL}_{2}(\mathbb{Q}(\sqrt{-3}))$ which implies that $C$ is soluble. Therefore $G / N \leq \operatorname{Out}(N) \simeq C_{2} \times C_{2}$. By Lemma 2.2.1. we know that $[G: N]=4$. So there is some $\alpha \in G \backslash N$ such that $\alpha$ induces the outer automorphism on $\mathrm{SL}_{2}(3)$ and acts trivially on $O_{3}(G)$. Hence $\alpha$ commutes with the center of $E:=\operatorname{End}(\bar{N}) \simeq \mathbb{Q}(\sqrt{-3})^{2 \times 2}$. By Lemma 2.2.16, $H:=\langle N, \alpha\rangle$ is (up to conjugacy) uniquely determined by the isomorphism type of $K:=C_{E}(\alpha)$. There are two possibilities, namely $K \simeq \mathbb{Q}(\sqrt{-3}, \sqrt{2})$ or $K \simeq \mathbb{Q}(\sqrt{-3}, \sqrt{-2})$.

- $K \simeq \mathbb{Q}(\sqrt{-3}, \sqrt{-2})$ : The maximal totally real subfield of $K$ is isomorphic to $\mathbb{Q}(\sqrt{6})$. Further $H$ fixes only one lattice $L$ and there exists some $F \in \mathcal{F}_{>0}(H)$ that is integral on $L$ such that $\operatorname{det}(L, F)=16$. Since $\operatorname{Nr}_{K / \mathbb{Q}(\sqrt{6})}\left(\mathbb{Z}_{K}^{*}\right)=\mathbb{Z}[\sqrt{6}]_{>0}^{*}$ we may restrict ourselves to one class of totally positive units. By Table 2.5.4, this leaves the following four candidates.

\begin{tabular}{|c|c|c|}
\hline form & $\mathbb{Q}(\sqrt{-3})$ & $\mathbb{Q}(\sqrt{-2})$ \\
\hline \hline$F$ & $\left({ }_{\infty, 2}\left[\mathrm{SL}_{2}(3)\right]_{1} \circ C_{3}\right)^{2}$ & $\sqrt{-2}\left[F_{4}: 2\right]_{2}$ \\
\hline $\mathfrak{p}_{3} F$ & $\sqrt{-3}\left[C_{6}\right]_{1} \otimes F_{4}$ & $\subsetneq \sqrt{-2}\left[F_{4}: 2\right]_{2}$ \\
\hline
\end{tabular}

But none of these groups has the correct Fitting subgroup.

- $K \simeq \mathbb{Q}(\sqrt{-3}, \sqrt{2})$ : The maximal totally real subfield of $K$ is isomorphic to $\mathbb{Q}(\sqrt{2})$. Further $H$ fixes only one lattice $L$ and there exists some $F \in \mathcal{F}_{>0}(H)$ which is integral on $L$ such that $\operatorname{det}(L, F)=1$. By Table 2.5.4, there are four candidates:

\begin{tabular}{|c|c|c|}
\hline form & $\mathbb{Q}(\sqrt{-3})$ & $\mathbb{Q}(\sqrt{-6})$ \\
\hline \hline$F$ & $\sqrt{-3}\left[\operatorname{Sp}_{4}(3) \circ C_{3}\right]_{4}$ & $\sqrt{-6}\left[\sqrt{2}, \infty\left[\tilde{S}_{4}\right]_{1}{ }^{2} \square C_{3}\right]_{4}$ \\
\hline $\mathfrak{p}_{2} F$ & $\left({ }_{\infty, 2}\left[\mathrm{SL}_{2}(3)\right]_{1} \circ C_{3}\right)^{2}$ & $\sqrt{-6}\left[\sqrt{2}, \infty\left[\tilde{S}_{4}\right]_{1}{ }^{2+} C_{3}\right]_{4}$ \\
\hline
\end{tabular}

The result follows if one checks the Fitting subgroups of these candidates. 
$O_{p}(G)=1$ for all odd primes $p$

In this last section, suppose that $F(G)=O_{2}(G)$. By Table 2.5.2, $O_{2}(G)$ is isomorphic to one of $C_{2}, C_{4}, C_{8}, C_{16}, D_{8}, Q_{8}, 2_{+}^{1+4}, 2_{-}^{1+4}, D_{8} \otimes C_{4}, D_{8} \otimes C_{8}, D_{8} \otimes Q D_{16}, 2_{+}^{1+4} \otimes C_{4}$, $D_{16}, Q D_{16}, Q_{16}$ or $Q D_{32}$. These cases are handled below. This concludes the classification of the s.i.m.f. matrix groups of degree 8 .

Lemma 4.5.16 If $F(G) \simeq 2_{+}^{1+4} \otimes C_{4}$, then $G=\mathcal{B}^{o}(F(G)) \simeq{ }_{i}\left[\left(2_{+}^{1+4} \otimes C_{4}\right) . S_{6}\right]_{4}$.

Proof: The group $\mathcal{B}^{o}(F(G))$ fixes up to isomorphism only one lattice $L$ and has $\mathbb{Q}(i)$ as commuting algebra. Hence the claim is easily verified.

Lemma 4.5.17 If $F(G) \simeq Q D_{32}$, then $G=F(G)$.

Proof: Follows from Lemma 3.3.1.

Lemma 4.5.18 $F(G)$ is not isomorphic to $C_{8}, D_{16}, Q D_{16}$ or $Q_{16}$.

Proof: In all these cases $G$ would contain a normal cyclic subgroup $N$ of order 8 . Then $C:=C_{G}(N)$ embeds into $\mathrm{GL}_{2}\left(\mathbb{Q}\left(\zeta_{8}\right)\right)$. Hence $E(G)=1$ and this implies that $G / F(G) \leq \operatorname{Out}(F(G))$ is a 2-group. So $G=F(G)$ is reducible.

Lemma 4.5.19 If $F(G) \simeq 2_{-}^{1+4}$, then $G$ is conjugate to $\sqrt{-2}\left[\infty, 2\left[2_{-}^{1+4} \text {. Alt }_{5}\right]_{2}: 2\right]_{4}$.

Proof: The normal subgroup $N:=\mathcal{B}^{\circ}(F(G)) \simeq{ }_{\infty, 2}\left[2_{-}^{1+4} \text {. Alt }\right]_{5}$ is self centralizing in $G$ and $\operatorname{End}(\bar{N}) \simeq Q_{\infty, 2}$. Hence $[G: N] \leq 2$. Thus by Remark 2.2.17, $G$ must contain a subgroup $U$ conjugate to $N .2$. Since $U$ fixes up to isomorphism a unique lattice and has $\mathbb{Q}(\sqrt{-2})$ as commuting algebra, the claim is easily verified.

Lemma 4.5.20 If $F(G) \simeq 2_{+}^{1+4}$, then $G$ is conjugate to $\sqrt{-2}\left[F_{4}: 2\right]_{4}$.

Proof: Let $N:=\mathcal{B}^{\circ}(F(G)) \simeq F_{4}$. Then $C:=C_{G}(N)$ embeds into $\mathbb{Q}^{2 \times 2}$. So $C$ and $G$ are soluble. Thus again $[G: N] \leq 2$ and by Remark 2.2.17 we conclude that $G \simeq \sqrt{-2}\left[F_{4}: 2\right]_{4}$. Finally one checks that this group is s.i.m.f..

Lemma 4.5.21 $F(G) \not C_{16}$.

Proof: Follows from Lemma 4.5.2. 
Lemma 4.5.22 $F(G)$ is neither isomorphic to $D_{8} \otimes C_{8}$ nor $D_{8} \otimes Q D_{16}$.

Proof: In both cases, $G$ would contain an irreducible normal subgroup $N \simeq D_{8} \otimes C_{8}$. Then $B:=\mathcal{B}^{\circ}(N) \simeq N . S_{3}$ fixes only one lattice $L$ and has $\mathbb{Q}\left(\zeta_{8}\right)$ as commuting algebra. One finds some $F \in \mathcal{F}_{>0}(B)$ that is integral on $L$ such that $\operatorname{det}(L, F)=1$. Since $\Pi(|G|)=\{2,3\}$ it follows from Table 2.5.4 that we have to check the following four candidates:

\begin{tabular}{|c|c|c|}
\hline & $\mathbb{Q}(i)$ & $\mathbb{Q}(\sqrt{-2})$ \\
\hline \hline$F$ & ${ }_{i}\left[\left(2_{+}^{1+4} \otimes C_{4}\right) \cdot S_{6}\right]_{4}$ & $\sqrt{-2}\left[\infty, 2\left[2_{-}^{1+4} \cdot \mathrm{Alt}_{5}\right]_{2}: 2\right]_{4}$ \\
\hline $\mathfrak{p}_{2} F$ & ${ }_{i}\left[\left(D_{8} \otimes C_{4}\right) \cdot S_{3}\right]_{2}^{2}$ & $\sqrt{-2}\left[F_{4}: 2\right]_{4}$ \\
\hline
\end{tabular}

None of these groups has the correct Fitting subgroup.

Lemma 4.5.23 $F(G) \not ॅ D_{8} \otimes C_{4}$.

Proof: Suppose $F(G) \simeq D_{8} \otimes C_{4}$. Then $N:=\mathcal{B}^{o}(F(G)) \simeq\left(D_{8} \otimes C_{4}\right) . S_{3}$ has $\mathbb{Q}(i)^{2 \times 2}$ as commuting algebra. Thus $E(G)=1$. But then $[G: N] \leq 2$ contradicts Lemma 2.2.1 $\square$

Lemma 4.5.24 $F(G) \not Q_{8}$.

Proof: Suppose $F(G) \simeq Q_{8}$. Then $N:=\mathcal{B}^{\circ}(F(G)) \simeq{ }_{\infty, 2}\left[\mathrm{SL}_{2}(3)\right]_{1}$ and $C_{G}(N)$ embeds into $\mathrm{GL}_{2}\left(\mathcal{Q}_{\infty, 2}\right)$. Hence it follows from Table 2.5.1 that $E(G)=1$. Thus $G / N \leq$ $\operatorname{Out}\left(\mathrm{SL}_{2}(3)\right) \simeq C_{2}$ contradicts Lemma 2.2.1.

\section{Lemma 4.5.25}

(a) If $F(G)$ is isomorphic to $C_{4}$, then $G$ is conjugate to $\sqrt{-5}\left[\sqrt{5}, \infty\left[\mathrm{SL}_{2}(5)\right]_{1} \stackrel{2_{+}}{\square} C_{4}\right]_{4}$ or $\sqrt{-5}\left[\sqrt{5}, \infty\left[\mathrm{SL}_{2}(5)\right]_{1}{ }^{2-} C_{4}\right]_{4}$.

(b) The Fitting group $F(G)$ is not isomorphic to $D_{8}$.

Proof: If $E(G)=1$ then $[G: F(G)] \leq 2$ contradicts Lemma 2.2.1. So by Table 2.5.1, $E(G)$ must be conjugate to $\mathrm{Alt}_{5}$ or $\mathrm{SL}_{2}(5)$. Thus the result follows from Lemmas 4.5.6 and 4.5.7.

Lemma 4.5.26 If $F(G) \simeq C_{2}$, then $G$ is conjugate to ${ }_{\sqrt{-7}}\left[2 . \mathrm{Alt}_{7}\right]_{4}$.

Proof: By Table 2.5.1, $E(G)$ is conjugate to one of $\mathrm{Alt}_{5}, \mathrm{SL}_{2}(5), \mathrm{SL}_{2}(7), \mathrm{SL}_{2}(9)$ or 2. Alt $_{7}$ (note that $\left.O_{3}\left(\mathcal{B}^{\circ}\left(\operatorname{Sp}_{4}(3)\right)\right) \simeq C_{3}\right)$. We have already classified these groups $G$ in Lemmas 4.5.6 and 4.5.7. 


\subsection{Dimension 10}

Theorem 4.6.1 The s.i.m.f. subgroups $G$ of $\operatorname{Sp}_{10}(\mathbb{Q})$ are

\begin{tabular}{|c|c|c|c|c|c|}
\hline$\#$ & $G$ & $|G|$ & $|\mathcal{Z}(G)|$ & $L_{\text {min }}$ & r.i.m.f. supergroups \\
\hline \hline$[2,1,5]$ & ${ }_{i}\left[C_{4}\right]_{1}^{5}$ & $2^{13} \cdot 3 \cdot 5$ & 3 & {$[1,1,20]$} & $B_{10}$ \\
\hline 1 & ${ }_{i}\left[C_{4}\right]_{1} \otimes A_{5}$ & $2^{6} \cdot 3^{2} \cdot 5$ & 6 & {$\left[3^{2}, 2,60\right]$} & $A_{5}^{2}$ \\
\hline \hline$[2,2,5]$ & ${ }_{-3}\left[C_{6}\right]_{1}^{5}$ & $2^{8} \cdot 3^{6} \cdot 5$ & 1 & {$\left[3^{5}, 2,30\right]$} & $A_{2}^{5}$ \\
\hline 2 & $\sqrt{-3}\left[ \pm S_{4}(3) \circ C_{3}\right]_{5}$ & $2^{7} \cdot 3^{5} \cdot 5$ & 2 & {$\left[2^{2} \cdot 3^{5}, 4,270\right]$} & {$\left[\left(C_{6} \times S_{4}(3)\right) .2\right]_{10}$} \\
\hline \hline 3 & $\sqrt{-11}\left[ \pm \mathrm{L}_{2}(11)\right]_{5}$ & $2^{3} \cdot 3 \cdot 5 \cdot 11$ & 1 & {$\left[11^{5}, 6,110\right]$} & $A_{10}^{(3)}$ \\
\hline
\end{tabular}

Proof: We know that that $\sqrt{-3}\left[C_{6}\right]_{1}^{5}$ is maximal finite by Lemma 2.1.21. The group ${ }_{i}\left[C_{4}\right]_{1}^{5}$ fixes three lattices and has $\mathbb{Q}(i)$ as commuting algebra. One checks that it is also maximal finite. So we may now suppose that $G$ is s.p.i.m.f..

Then $G$ cannot be soluble according to Corollary 4.1.2. So Table 2.5.1 shows that $E(G)$ is isomorphic to $\mathrm{Alt}_{6}, \mathrm{~L}_{2}(11)$ or $S_{4}(3)$.

The group $\sqrt{-11}\left[ \pm \mathrm{L}_{2}(11)\right]_{5}$ is s.i.m.f. by Theorem 3.1.1.

If $E(G) \simeq S_{4}(3)$, then $\operatorname{End}(\bar{N}) \simeq \mathbb{Q}(\sqrt{-3})$ and $\mathcal{B}^{\circ}(E(G)) \simeq \sqrt{-3}\left[ \pm S_{4}(3) \circ C_{3}\right]_{5}$ fixes up to isomorphism one lattice $L$. One immediately verifies that it is maximal finite.

Suppose now $E(G) \simeq \operatorname{Alt}_{6}$. Then $N:=\mathcal{B}^{\circ}(E(G)) \simeq \operatorname{Aut}\left(A_{5}\right) \simeq \pm S_{6}$. If $F(G)= \pm I_{10}$ then $G=N$ is reducible, since the exceptional outer automorphism of $S_{6}$ cannot be realized in $\mathrm{GL}_{5}(\mathbb{C})$. So $F(G)$ is cyclic of order 6 or 4 by Lemma 4.1.1. In the first case $G$ would be properly contained in $\sqrt{-3}\left[ \pm S_{4}(3) \circ C_{3}\right]_{5}$. In the latter case $G={ }_{i}\left[C_{4}\right]_{1} \otimes A_{5}$ which one easily verifies to be maximal finite by computing the automorphism groups of all six $G$-invariant lattices.

The r.i.m.f. supergroups are easily constructed since $G$ is uniform in any case. 


\subsection{Dimension 12}

Theorem 4.7.1 The s.i.m.f. subgroups $G$ of $\operatorname{Sp}_{12}(\mathbb{Q})$ are

\begin{tabular}{|c|c|c|c|c|c|}
\hline$\#$ & $G$ & $|G|$ & $|\mathcal{Z}(G)|$ & $L_{\min }$ & $\begin{array}{c}\text { r.i.m.f. } \\
\text { supergroups }\end{array}$ \\
\hline $\mid[4,1,3]$ & $\bar{c}{ }_{i}\left[\left(D_{8} \otimes C_{4}\right) \cdot S_{3}\right]_{2}^{3}$ & $22^{16} \cdot 3^{4}$ & 1 & $\overline{\left[2^{6}, 2,72\right]}$ & $\overline{F_{4}^{3}}$ \\
\hline$[2,1,6]$ & ${ }_{i}\left[C_{4}\right]_{1}^{6}$ & $2^{16} \cdot 3^{2} \cdot 5$ & 3 & {$[1,1,24]$} & $B_{12}$ \\
\hline 1 & ${ }_{i}\left[C_{4}\right]_{1} \otimes E_{6}$ & $2^{9} \cdot 3^{4} \cdot 5$ & 2 & {$\left[3^{2}, 2,144\right]$} & $E_{6}^{2}$ \\
\hline$[4,2,3]$ & $\left({ }_{i}\left[C_{4}\right]_{1} \otimes A_{2}\right)^{3}$ & $2^{10} \cdot 3^{4}$ & 2 & {$\left[3^{6}, 2,36\right]$} & $A_{2}^{6}$ \\
\hline 2 & $\infty, 3\left[ \pm U_{3}(3)\right]_{3} \circ C_{4}$ & $2^{7} \cdot 3^{3} \cdot 7$ & 2 & {$\left[3^{6}, 4,756\right]$} & {$\left[6 . U_{4}(3) .2^{2}\right]_{12}$} \\
\hline 3 & ${ }_{i}\left[C_{4}\right]_{1} \otimes A_{6}$ & $2^{6} \cdot 3^{2} \cdot 5 \cdot 7$ & 2 & {$\left[7^{2}, 2,84\right]$} & $A_{6}^{2}$ \\
\hline 4 & ${ }_{i}\left[S^{2(2)}{ }_{i}\left[C_{4}\right]_{1}\right]_{6}$ & $2^{6} \cdot 3^{4}$ & 4 & {$\left[2^{6} \cdot 3^{4}, 4,216\right]$} & {$\left[S \underset{\sqrt{-3}}{\stackrel{2}{\bigotimes}} \mathrm{SL}_{2}(3)\right]_{12}$} \\
\hline 5 & ${ }_{i}\left[\mathrm{~L}_{2}(7)^{2(2)} \otimes_{i}\left[C_{4}\right]_{1}\right]_{6}$ & $2^{6} \cdot 3 \cdot 7$ & 2 & {$\left[2^{6} \cdot 7^{2}, 4,336\right]$} & {$\left[\mathrm{L}_{2}(7)^{2(2)} \otimes D_{8}\right]_{12}$} \\
\hline 6 & ${ }_{i}\left[C_{4}\right]_{1} \otimes A_{6}^{(2)}$ & $2^{6} \cdot 3 \cdot 7$ & 2 & {$\left[7^{6}, 4,84\right]$} & $\left(A_{6}^{(2)}\right)^{2}$ \\
\hline 7 & $i\left[\sqrt{-7}\left[ \pm \mathrm{L}_{2}(7)\right]_{3}^{2(2)} \otimes_{i}\left[C_{4}\right]_{1}\right]_{6}$ & $2^{6} \cdot 3 \cdot 7$ & 2 & {$\left[2^{6} \cdot 7^{6}, 8,336\right]$} & {$\left[\mathrm{L}_{2}(7) \stackrel{2(2)}{\otimes} D_{8}\right]_{12}$} \\
\hline$[4,3,3]$ & $\overline{\sqrt{-2}}\left[\mathrm{GL}_{2}(3)\right]_{2}^{3}$ & $2^{13} \cdot 3^{4}$ & 1 & $\overline{\left[2^{6}, 2,72\right]}$ & $\overline{\bar{F}} \bar{F}_{4}^{3}$ \\
\hline 8 & $\sqrt{-2}\left[ \pm \mathrm{L}_{2}(7) \cdot 2\right]_{6}$ & $2^{5} \cdot 3 \cdot 7$ & 2 & {$\left[7^{2}, 2,84\right]$} & $A_{6}^{2}$ \\
\hline 9 & $\sqrt{-2}\left[\infty, 2\left[\mathrm{SL}_{2}(5)\right]_{3}: 2\right]_{6}$ & $2^{4} \cdot 3 \cdot 5$ & 6 & {$\left[2^{2} \cdot 5,3,80\right]$} & {$\left[\mathrm{SL}_{2}(5)^{2(2)} \circ \mathrm{SL}_{2}(3)\right]_{12}$} \\
\hline 10 & $\sqrt{-3}\left[6 . U_{4}(3) \cdot 2\right]_{6}$ & $2^{9} \cdot 3^{7} \cdot 5 \cdot 7$ & 1 & {$\left[3^{6}, 4,756\right]$} & {$\left[6 . U_{4}(3) .2^{2}\right]_{12}$} \\
\hline$[2,2,6]$ & $\sqrt{-3}\left[C_{6}\right]_{1}^{6}$ & $2^{10} \cdot 3^{8} \cdot 5$ & 1 & {$\left[3^{6}, 2,36\right]$} & $A_{2}^{6}$ \\
\hline$[6,1,2]$ & $\sqrt{-3}\left[ \pm 3_{+}^{1+2}: \mathrm{SL}_{2}(3)\right]_{3}^{2}$ & $2^{9} \cdot 3^{8}$ & 2 & {$\left[3^{2}, 2,144\right]$} & $E_{6}^{2}$ \\
\hline$[4,4,3]$ & $\left(\infty, 2\left[\mathrm{SL}_{2}(3)\right]_{1} \circ C_{3}\right)^{3}$ & $2^{10} \cdot 3^{7}$ & 2 & {$\left[2^{6}, 2,72\right]$} & $F_{4}^{3}$ \\
\hline 11 & $S \underset{\sqrt{-3}}{\otimes} \infty, 2\left[\mathrm{SL}_{2}(3)\right]_{1}$ & $2^{6} \cdot 3^{5}$ & 4 & {$\left[2^{6} \cdot 3^{4}, 4,216\right]$} & {$\left[S \underset{\sqrt{-3}}{\stackrel{2}{\otimes}} \mathrm{SL}_{2}(3)\right]_{12}$} \\
\hline 12 & $\sqrt{-3}\left[ \pm 3 . M_{10}\right]_{6}$ & $2^{5} \cdot 3^{3} \cdot 5$ & 2 & {$\left[3^{6} \cdot 5^{6}, 8,270\right]$} & {$\left[ \pm 3 . \mathrm{Alt}_{12} \cdot 2^{2}\right]_{12}$} \\
\hline 13 & $\sqrt{-3}\left[C_{6}\right]_{1} \otimes M_{6,2}$ & $2^{4} \cdot 3^{2} \cdot 5$ & 6 & {$\left[3^{6} \cdot 5^{6}, 6,60\right]$} & $A_{2} \otimes M_{6,2}$ \\
\hline 14 & $\infty, 2\left[\mathrm{SL}_{2}(5)\right]_{3} \circ C_{3}$ & $2^{3} \cdot 3^{2} \cdot 5$ & 8 & {$\left[2^{2} \cdot 5^{4}, 4,360\right]$} & {$\left[\mathrm{SL}_{2}(5)^{2(2)} \circ \mathrm{SL}_{2}(3)\right]_{12}$} \\
\hline 15 & $\sqrt{-5}\left[{ }_{i}\left[C_{4}\right]_{1} \stackrel{2_{-}}{\otimes} \mathrm{Alt}_{5}\right]_{6}$ & $2^{5} \cdot 3 \cdot 5$ & 14 & $\begin{array}{c}{[1,1,24]} \\
{[1,2,264]}\end{array}$ & $B_{12}$ \\
\hline 16 & $\sqrt{-5}\left[i\left[C_{4}\right]_{1} \stackrel{2_{+}}{\otimes} \mathrm{Alt}_{5}\right]_{6}$ & $2^{5} \cdot 3 \cdot 5$ & 14 & $\begin{array}{l}{\left[5^{6}, 4,60\right]} \\
{\left[5^{6}, 3,40\right]}\end{array}$ & $M_{6,2}^{2}$ \\
\hline$[6,2,2]$ & $\sqrt{-7}\left[ \pm \mathrm{L}_{2}(7)\right]_{3}^{2}$ & $2^{9} \cdot 3^{2} \cdot 7^{2}$ & 1 & {$\left[7^{2}, 4,84\right]$} & $\left(A_{6}^{(2)}\right)^{2}$ \\
\hline 17 & $\sqrt{-7}\left[ \pm \mathrm{L}_{2}(7)\right]_{3} \underset{\sqrt{-7}}{\otimes} \infty, 3\left[\tilde{S}_{3}\right]_{1}$ & $2^{5} \cdot 3^{2} \cdot 7$ & 2 & {$\left[3^{6}, 4,756\right]$} & {$\left[6 . U_{4}(3) .2^{2}\right]_{12}$} \\
\hline 18 & $\sqrt{-7}\left[ \pm \mathrm{L}_{2}(7)\right]_{3} \otimes A_{2}$ & $2^{5} \cdot 3^{2} \cdot 7$ & 2 & {$\left[3^{6} \cdot 7^{6}, 8,126\right]$} & $A_{2} \otimes A_{6}^{(2)}$ \\
\hline 19 & $\sqrt{-11}\left[\mathrm{SL}_{2}(11)\right]_{6}$ & $2^{3} \cdot 3 \cdot 5 \cdot 11$ & 5 & $\begin{array}{c}{[1,1,24]} \\
{[1,2,264]}\end{array}$ & $B_{12}$ \\
\hline 20 & $\sqrt{-15}\left[ \pm 3 . \mathrm{Alt}_{6} \cdot 2_{1}\right]_{6}$ & $2^{5} \cdot 3^{3} \cdot 5$ & 2 & {$\left[3^{6}, 4,756\right]$} & {$\left[6 . U_{4}(3) .2^{2}\right]_{12}$} \\
\hline 21 & $\sqrt{-15}\left[ \pm 3 . \text { Alt }_{6}: 2_{1}\right]_{6}$ & $2^{5} \cdot 3^{3} \cdot 5$ & 2 & {$\left[3^{6} \cdot 5^{6}, 8,270\right]$} & {$\left[ \pm 3 . \text { Alt }_{12} \cdot 2^{2}\right]_{12}$} \\
\hline$[4,5,3]$ & $\zeta_{10}\left[C_{10}\right]_{1}^{3}$ & $2^{4} \cdot 3 \cdot 5^{3}$ & $\overline{1}$ & {$\left[5^{3}, 2,60\right]$} & $\overline{A_{4}^{3}}$ \\
\hline
\end{tabular}




\begin{tabular}{|c|c|c|c|c|c|}
\hline 22 & $\zeta_{10}\left[C_{10}\right]_{1} \otimes \mathrm{Alt}_{5}$ & $2^{3} \cdot 3 \cdot 5^{2}$ & 2 & {$\left[2^{4} \cdot 5^{3}, 4,420\right]$} & {$\left[D_{20} \underset{\sqrt{5}}{\stackrel{2}{\otimes}} \mathrm{Alt}_{5}\right]_{12}$} \\
\hline \hline 23 & $\zeta_{26}+\zeta_{26}^{3}+\zeta_{26}^{9}\left[C_{26}: C_{3}\right]_{3}$ & $2 \cdot 3 \cdot 13$ & 9 & {$[13,2,156]$} & $A_{12}$ \\
\hline
\end{tabular}

Here $S$ denotes the s.p.i.m.f. subgroup $\sqrt{-3}\left[ \pm 3_{+}^{1+2}: \mathrm{SL}_{2}(3)\right]_{3}<\mathrm{Sp}_{6}(\mathbb{Q})$. A proof of this theorem is given in Section 4.7.2.

\subsubsection{Irreducible cyclic subgroups}

Let $G<\operatorname{Sp}_{12}(\mathbb{Q})$ be s.i.m.f. such that $G$ contains an irreducible cyclic subgroup $U$. Then $| \pm U| \in\{26,28,36,42\}$. These groups are classified below.

Lemma 4.7.2 If $\pm U$ has order 26 then $G$ is conjugate to $\zeta_{26}+\zeta_{26}^{3}+\zeta_{26}^{9}\left[C_{26}: C_{3}\right]_{3}$.

Proof: Follows from Theorem 3.1.1.

Lemma 4.7.3 If $U$ has order 28 , then $G$ is conjugate to ${ }_{i}\left[C_{4}\right]_{1} \otimes A_{6},{ }_{i}\left[C_{4}\right]_{1} \otimes A_{6}^{(2)}$, ${ }_{i}\left[\mathrm{~L}_{2}(7)_{6} \stackrel{2(2)}{\otimes}_{i}\left[C_{4}\right]_{1}\right]_{6},{ }_{i}\left[\sqrt{-7}\left[ \pm \mathrm{L}_{2}(7)\right]_{3} \stackrel{2(2)}{\otimes}{ }_{i}\left[C_{4}\right]_{1}\right]_{6}$ or ${ }_{\sqrt{-7}}\left[ \pm \mathrm{L}_{2}(7)\right]_{3}^{2}$.

Proof: The commuting algebra of $U$ is isomorphic to $\mathbb{Q}\left(\zeta_{28}\right)$ and has class number 1. Thus $U$ fixes only one lattice $L$. Further there exists some $F \in \mathcal{F}_{>0}(U)$ such that $F$ is integral on $L$ and $\operatorname{det}(L, F)=7^{2}$. It follows from Minkowski's bound that $\Pi(|G|)=\{2,3,5,7,11,13\}$. The cases that $|G|$ is divisible by 11 or 13 are handled in Theorems 3.2.1 and 3.1.1. So we have $\Pi(|G|)=\{2,3,5,7\}$. By Table 2.5.3. $G$ is conjugate to $\operatorname{Aut}_{\mathbb{Q}(\sqrt{-d})}\left(L, \mathfrak{p}_{2}^{k} \mathfrak{p}_{7}^{2 l} F\right)$ for some $k, l \in\{0,1\}$ and $d \in\{1,7\}$. These automorphism groups are

\begin{tabular}{|c|c|c|}
\hline form & $\mathbb{Q}(i)$ & $\mathbb{Q}(\sqrt{-7})$ \\
\hline \hline$F$ & ${ }_{i}\left[C_{4}\right]_{1} \otimes A_{6}$ & $\subsetneq \sqrt{-7}\left[ \pm \mathrm{L}_{2}(7)\right]_{3}^{2}$ \\
\hline $\mathfrak{p}_{2} F$ & ${ }_{i}\left[\mathrm{~L}_{2}(7)_{6}{ }^{2(2)} \otimes_{i}\left[C_{4}\right]_{1}\right]_{6}$ & $\subsetneq \sqrt{-7}\left[ \pm \mathrm{L}_{2}(7)\right]_{3}^{2}$ \\
\hline $\mathfrak{p}_{7}^{2} F$ & ${ }_{i}\left[C_{4}\right]_{1} \otimes A_{6}^{(2)}$ & $\sqrt{-7}\left[ \pm \mathrm{L}_{2}(7)\right]_{3}^{2}$ \\
\hline $\mathfrak{p}_{2} \mathfrak{p}_{7}^{2} F$ & ${ }_{i}\left[{ }_{\sqrt{-7}}\left[ \pm \mathrm{L}_{2}(7)\right]_{3}{ }^{2(2)}{ }_{i}\left[C_{4}\right]_{1}\right]_{6}$ & $\subsetneq \sqrt{-7}\left[ \pm \mathrm{L}_{2}(7)\right]_{3}^{2}$ \\
\hline
\end{tabular}

So the result follows.

Lemma 4.7.4 If $U$ has order 36 , then $G$ is conjugate to one of:

$$
\begin{gathered}
{ }_{i}\left[C_{4}\right]_{1} \otimes E_{6}, \sqrt{-3}\left[ \pm 3_{+}^{1+2}: \mathrm{SL}_{2}(3)\right]_{3}^{2},{ }_{i}\left[{ }_{\sqrt{-3}}\left[ \pm 3_{+}^{1+2}: \mathrm{SL}_{2}(3)\right]_{3} \stackrel{2(2)}{\otimes}{ }_{i}\left[C_{4}\right]_{1}\right]_{6}, \\
\sqrt{-3}\left[ \pm 3_{+}^{1+2}: \mathrm{SL}_{2}(3)\right]_{3} \otimes \infty, 2\left[\mathrm{SL}_{2}(3)\right]_{1}, \quad\left({ }_{i}\left[C_{4}\right]_{1} \otimes A_{2}\right)^{2}, \sqrt{-3}\left[C_{6}\right]_{1}^{6} .
\end{gathered}
$$


Proof: The commuting algebra of $U$ is isomorphic to $\mathbb{Q}\left(\zeta_{36}\right)$ and has class number 1 . Thus $U$ fixes only one lattice $L$. Further there exists some $F \in \mathcal{F}_{>0}(U)$ such that $F$ is integral on $L$ and $\operatorname{det}(L, F)=9$. As above, we have $\Pi(|G|) \subseteq\{2,3,5,7\}$. Restricting to normalized lattices (see Definition 2.2.4), Table 2.5.3 shows that $G$ is conjugate to $\operatorname{Aut}_{\mathbb{Q}(\sqrt{-d})}\left(L, \mathfrak{p}_{2}^{k} \mathfrak{p}_{3}^{l} F\right)$ for some $(k, l) \in\{(0,0),(1,1),(0,2)\}$ and $d \in\{1,3\}$. These groups are given below.

\begin{tabular}{|c|c|c|}
\hline form & $\mathbb{Q}(i)$ & $\mathbb{Q}(\sqrt{-3})$ \\
\hline \hline$F$ & ${ }_{i}\left[C_{4}\right]_{1} \otimes E_{6}$ & $\sqrt{-3}\left[ \pm 3_{+}^{1+2}: \mathrm{SL}_{2}(3)\right]_{3}^{2}$ \\
\hline $\mathfrak{p}_{2} \mathfrak{p}_{3} F$ & ${ }_{i}\left[{ }_{\sqrt{-3}}\left[ \pm 3_{+}^{1+2}: \mathrm{SL}_{2}(3)\right]_{3} \otimes{ }_{i}\left[C_{4}\right]_{1}\right]_{6}$ & $\sqrt{-3}\left[ \pm 3_{+}^{1+2}: \mathrm{SL}_{2}(3)\right]_{3} \otimes \otimes_{\infty, 2}\left[\mathrm{SL}_{2}(3)\right]_{1}$ \\
\hline $\mathfrak{p}_{3}^{2} F$ & $\left({ }_{i}\left[C_{4}\right]_{1} \otimes A_{2}\right)^{2}$ & $\sqrt{-3}\left[C_{6}\right]_{1}^{6}$ \\
\hline
\end{tabular}

This proves the result.

Lemma 4.7.5 If $\pm U$ has order 42 , then $G$ is conjugate to one of

$$
\sqrt{-3}\left[6 . U_{4}(3) .2\right]_{6}, \sqrt{-7}\left[ \pm \mathrm{L}_{2}(7)\right]_{3} \otimes A_{2}, \sqrt{-7}\left[ \pm \mathrm{L}_{2}(7)\right]_{3} \underset{\sqrt{-7}}{\otimes} \infty\left[3\left[\tilde{S}_{3}\right]_{1}\right.
$$

Proof: The commuting algebra of $U$ is isomorphic to $\mathbb{Q}\left(\zeta_{42}\right)$ and has class number 1 . So $U$ fixes only one lattice $L$. Further one finds some $F \in \mathcal{F}_{>0}(U)$ such that $F$ is integral on $L$ and $\operatorname{det}(L, F)=3^{6}$. As above we have $\Pi(|G|)=\{2,3,5,7\}$. By Table 2.5.3, $G$ is conjugate to $\operatorname{Aut}_{\mathbb{Q}(\sqrt{-d})}\left(L, \mathfrak{p}_{3}^{-k} \mathfrak{p}_{5}^{k} \mathfrak{p}_{7}^{l} F\right)$ or $\operatorname{Aut}_{\mathbb{Q}(\sqrt{-d})}\left(L, \mathfrak{p}_{3}^{-k} \mathfrak{p}_{5}^{\prime k} \mathfrak{p}_{7}^{l} F\right)$ for some $k \in\{0,1\}$, $0 \leq l \leq 3$ and $d \in\{3,7\}$. Since $\operatorname{Aut}_{\mathbb{Q}(\sqrt{-d})}\left(L, \mathfrak{p}_{3}^{-1} \mathfrak{p}_{5}^{\prime} \mathfrak{p}_{7}^{l} F\right)=\operatorname{Aut}_{\mathbb{Q}(\sqrt{-d})}\left(L, \mathfrak{p}_{3}^{-1} \mathfrak{p}_{5} \mathfrak{p}_{7}^{l} F\right)=$ $\operatorname{Aut}_{\mathbb{Q}(\sqrt{-3})}\left(L, \mathfrak{p}_{7}^{l} F\right) \cap \operatorname{Aut}_{\mathbb{Q}(\sqrt{-7})}\left(L, \mathfrak{p}_{7}^{l} F\right)$ for all $l$ and $d$ we have eight groups to check.

\begin{tabular}{|c|c|c|}
\hline form & $\mathbb{Q}(\sqrt{-3})$ & $\mathbb{Q}(\sqrt{-7})$ \\
\hline$F$ & $\sqrt{-3}\left[6 \cdot U_{4}(3) \cdot 2\right]_{6}$ & $\sqrt{-7}\left[ \pm \mathrm{L}_{2}(7)\right]_{3} \underset{\sqrt{-7}}{\otimes} \infty, 3\left[\tilde{S}_{3}\right]_{1}$ \\
\hline $\mathfrak{p}_{7} F$ & $\lesseqgtr \sqrt{-3}\left[6 . U_{4}(3) .2\right]_{6}$ & $\lesseqgtr \sqrt{-7}\left[ \pm \mathrm{L}_{2}(7)\right]_{3} \otimes A_{2}$ \\
\hline $\mathfrak{p}_{7}^{2} F$ & $\lesseqgtr \sqrt{-3}\left[6 . U_{4}(3) \cdot 2\right]_{6}$ & $\lesseqgtr \sqrt{-7}\left[ \pm \mathrm{L}_{2}(7)\right]_{3} \underset{\sqrt{-7}}{\otimes} \infty, 3\left[\tilde{S}_{3}\right]_{1}$ \\
\hline $\mathfrak{p}_{7}^{3} F$ & $\lesseqgtr_{\sqrt{-3}}\left[6 . U_{4}(3) \cdot 2\right]_{6}$ & $\left.-7 \pm \mathrm{L}_{2}(7)\right]_{3} \otimes A_{2}$ \\
\hline
\end{tabular}

So the lemma is proven.

\subsubsection{Proof of Theorem 4.7.1}

We have to prove the completeness of the list given in Theorem 4.7.1 (the correctness follows from explicit calculations). The candidates for the maximal finite symplectic imprimitive groups come from the classification of the s.p.i.m.f. subgroups of $\mathrm{Sp}_{2}(\mathbb{Q}), \mathrm{Sp}_{4}(\mathbb{Q})$ and $\mathrm{Sp}_{6}(\mathbb{Q})$. By Lemma 2.1.21, we only have to check the group ${ }_{i}\left[C_{4}\right]_{1}^{6}$. It fixes up to isomorphism three lattices and has $\mathbb{Q}(i)$ as commuting algebra. One verifies that it is s.i.m.f..

So it remains to prove the completeness for the s.p.i.m.f. matrix groups. Thus, for the remainder of this section let $G<\operatorname{Sp}_{12}(\mathbb{Q})$ be s.p.i.m.f.. 
The case that $O_{13}(G) \neq 1$ is handled in Lemma 4.7.2. Hence we may assume that $F(G)=O_{2}(G) O_{3}(G) O_{5}(G)$. So Theorem 4.7 .1 follows by discussing all possible candidates for the Fitting subgroup of $G$. This is done in the subsequent lemmas.

Again, we handle the irreducible quasisimple normal subgroups first.

Lemma 4.7.6 Suppose $G<\operatorname{Sp}_{12}(\mathbb{Q})$ is s.p.i.m.f. and $G$ contains an irreducible quasisimple normal subgroup $N$. Then one of the following holds.

(a) If $N \simeq 6 . U_{4}(3)$ then $G=\mathcal{B}^{\circ}(N) \simeq \sqrt{-3}\left[6 \cdot U_{4}(3) \cdot 2\right]_{6}$.

(b) If $N \simeq U_{3}(3)$ then $G \simeq \infty, 3\left[ \pm U_{3}(3)\right]_{3} \circ C_{4}$.

(c) If $N$ is conjugate to 3 . Alt ${ }_{6}$ with character $\chi_{3 a}+\chi_{3 a}^{\prime}+\chi_{3 b}+\chi_{3 b}^{\prime}$, then $G$ is conjugate to $\sqrt{-15}\left[ \pm 3 . \mathrm{Alt}_{6} \cdot 2\right]_{6}, \sqrt{-15}\left[ \pm 3 . \mathrm{Alt}_{6}: 2\right]_{6}$ or $\sqrt{-3}\left[ \pm 3 . M_{10}\right]_{6}$.

(d) If $N \simeq \mathrm{SL}_{2}(11)$ then $G=N$.

(e) If $N$ is conjugate to $\infty_{, 2}\left[\mathrm{SL}_{2}(5)\right]_{3}$, then $G$ is conjugate to $\sqrt{-2}\left[\infty, 2\left[\mathrm{SL}_{2}(5)\right]_{3}: 2\right]_{6}$ or $\infty, 2\left[\mathrm{SL}_{2}(5)\right]_{3} \circ C_{3}$.

Proof: Suppose first that we are in one of the cases mentioned above.

(a) $\mathcal{B}^{\circ}(N)$ fixes only one lattice and has $\mathbb{Q}(\sqrt{-3})$ as commuting algebra. So the claim is easily verified.

(b) The commuting algebra of $N$ is isomorphic to $\mathcal{Q}_{\infty, 3}$ and $\operatorname{Out}(N) \simeq C_{2}$. Thus by Remark 2.2.17, $G$ contains $N \circ C_{4}, N \circ C_{3}$ or $\pm N .2$ as a subgroup. The first group is already s.i.m.f.. The other two groups fix 1 or 4 lattices respectively. One checks that they are only contained in ${ }_{\sqrt{-3}}\left[6 . U_{4}(3) .2\right]_{6}$.

(c) The commuting algebra $C$ of $N$ is isomorphic to $\mathbb{Q}(\sqrt{-3}, \sqrt{5})$ with $\mathbb{Q}(\sqrt{5})$ as maximal totally real subfield. Further, $N$ fixes only one lattice $L$ and one finds some $F \in \mathcal{F}_{>0}(G)$ that is integral on $L$ with $\operatorname{det}(L, F)=3^{6}$. Since $\Pi(|G|)=$ $\{2,3,5\}$ this leaves the following 4 candidates (see Table 2.5.4).

\begin{tabular}{|c|c|c|}
\hline form & $\mathbb{Q}(\sqrt{-3})$ & $\mathbb{Q}(\sqrt{-15})$ \\
\hline \hline$F$ & $\sqrt{-3}\left[6 . U_{4}(3) \cdot 2\right]_{6}$ & $\sqrt{-3}\left[ \pm 3 . \mathrm{Alt}_{6} \cdot 2\right]_{6}$ \\
\hline $\mathfrak{p}_{5} F$ & $\sqrt{-3}\left[ \pm 3 . M_{10}\right]_{6}$ & $\sqrt{-3}\left[ \pm 3 . \mathrm{Alt}_{6}: 2\right]_{6}$ \\
\hline
\end{tabular}

So the result follows.

(d) Follows from Theorem 3.2.1.

(e) The centralizing algebra of $N$ is isomorphic to $Q_{\infty, 2}$ and $\operatorname{Out}(N) \simeq C_{2}$. Hence, by Remark 2.2.17, $G$ contains a subgroup conjugate to: $N \circ C_{3}, N .2$ and $N \circ C_{4}$. These fix 8,6 and 20 lattices respectively. The first two groups are maximal finite. The remaining group is only contained in $G={ }_{i}\left[C_{4}\right]_{1}^{6}$. 
If $N$ is not conjugate to one of these groups, it follows from Table 2.5.1, that $N$ must be conjugate to $6 . L_{3}(4)$ or $3 . \mathrm{Alt}_{6}$ with character $\chi_{6}+\chi_{6}^{\prime}$.

The group $6 . L_{3}(4)$ fixes two lattices and has $\mathbb{Q}(\sqrt{-3})$ as commuting algebra. One checks that it is only contained in $\sqrt{-3}\left[6 . U_{4}(3) .2\right]_{6}$.

In the remaining case, $N$ fixes 12 lattices and has $\mathbb{Q}(\sqrt{-3})$ as commuting algebra. One checks that $N$ has the maximal finite symplectic supergroups $\sqrt{-3}\left[6 . U_{4}(3) .2\right]_{6}$ and $\sqrt{-3}\left[C_{6}\right]_{1}^{6}$ which both do not normalize $N$.

Lemma 4.7.7 If $O_{5} \neq 1$ then $G$ is conjugate to $\zeta_{10}\left[C_{10}\right]_{1} \underset{\sqrt{5}}{\otimes \mathrm{Alt}_{5}}$.

Proof: By Table 2.5.2, $O_{5}(G) \simeq C_{5}$. Thus $C_{G}\left(O_{5}(G)\right)$ embeds into $\mathrm{GL}_{3}\left(\mathbb{Q}\left(\zeta_{10}\right)\right)$. In particular, by loc. cit. $F(G) \simeq C_{10}$. But then $E(G)=1$ contradicts Corollary 2.2.3. So Table 2.5.1 shows that $F^{*}(G) \simeq{ }_{\zeta_{10}}\left[C_{10}\right]_{1} \otimes{ }_{\sqrt{5}} \operatorname{Alt}_{5}$. But then $F^{*}(G)$ fixes 2 lattices and has $\mathbb{Q}\left(\zeta_{10}\right)$ as commuting algebra. One checks that it is already s.i.m.f. by computing their automorphism group (with respect to the full form space).

Lemma 4.7.8 If $\mathrm{O}_{3}(G)=3_{+}^{1+2}$, then $G$ is conjugate to one of

$$
\sqrt{-3}\left[ \pm 3_{+}^{1+2}: \mathrm{SL}_{2}(3)\right]_{3} \underset{\sqrt{-3}}{\otimes} \infty, 2\left[\mathrm{SL}_{2}(3)\right]_{1} \quad \text { or } \quad i\left[\sqrt{-3}\left[ \pm 3_{+}^{1+2}: \mathrm{SL}_{2}(3)\right]_{3} \stackrel{2(2)}{\bigotimes}{ }_{i}\left[C_{4}\right]_{1}\right]_{6}
$$

Proof: By Table 2.5.1 we have $E(G)=1$. If $F(G)= \pm O_{3}(G)$ then $G / \mathcal{B}^{\circ}(F(G)) \leq C_{2}$ contradicts Lemma 2.2.1. So by Table 2.5.2 we have $F(G) \simeq 3_{+}^{1+2} \otimes H$ with $H=C_{4}, D_{8}$ or $Q_{8}$. If $O_{2}(G) \simeq Q_{8}$, then $G=\mathcal{B}^{\circ}(\overline{F(G)}) \simeq \sqrt{-3}\left[ \pm 3_{+}^{1+2}: \mathrm{SL}_{2}(3)\right]_{3} \underset{\sqrt{-3}}{\otimes} \infty_{2}\left[\mathrm{SL}_{2}(3)\right]_{1}$ is already s.i.m.f..

So we may now assume that $G$ contains a normal subgroup conjugate to $N:=$ $B\left(O_{3}(G)\right) \otimes C_{4} \simeq \sqrt{-3}\left[3_{+}^{1+2}: \mathrm{SL}_{2}(3)\right]_{3} \otimes C_{4}$ of index at most 4 . In particular $\Pi(|G|)=$ $\{2,3\}$. The commuting algebra $C$ of $U$ is isomorphic to $\mathbb{Q}(i, \sqrt{3})$ and has $K \simeq \mathbb{Q}(\sqrt{3})$ as maximal totally real subfield. Up to isomorphism, $N$ fixes two lattices, which are both $N$-normal critical. Further we find some $L \in \mathcal{Z}(N)$ and a form $F \in \mathcal{F}_{>0}(U)$ that is integral on $L$ such that $\operatorname{det}(L, F)=3^{2}$. Since $\mathbb{Z}_{K,>0}^{*}=\mathrm{Nr}_{C / K}\left(\mathbb{Z}_{C}^{*}\right)$ we may restrict ourselves to one class of totally positive units. By Table 2.5.4, this leaves the following cases:

\begin{tabular}{|c|c|c|}
\hline form & $\mathbb{Q}(i)$ & $\mathbb{Q}(\sqrt{-3})$ \\
\hline \hline$F$ & ${ }_{i}\left[C_{4}\right]_{1} \otimes E_{6}$ & $\sqrt{-3}\left[ \pm 3_{+}^{1+2}: \mathrm{SL}_{2}(3)\right]^{2}$ \\
\hline $\mathfrak{p}_{2} \mathfrak{p}_{3} F$ & ${ }_{i}\left[\sqrt{-3}\left[ \pm 3_{+}^{1+2}: \mathrm{SL}_{2}(3)\right]_{3}{ }^{(2)}{ }_{i}\left[C_{4}\right]_{1}\right]_{6}$ & $\sqrt{-3}\left[ \pm 3_{+}^{1+2}: \mathrm{SL}_{2}(3)\right]_{3} \otimes_{\sqrt{-3}}{ }^{\infty, 2}\left[\mathrm{SL}_{2}(3)\right]_{1}$ \\
\hline
\end{tabular}

This proves the claim. 
Lemma 4.7.9 $\mathrm{O}_{3}(G)$ is not isomorphic to $C_{9}$.

Proof: Suppose $O_{3}(G)=C_{9}$. Then $C_{G}\left(O_{3}(G)\right)$ embeds into $\mathrm{GL}_{2}\left(\mathbb{Q}\left(\zeta_{9}\right)\right)$. Thus $G$ is soluble. If $F(G)= \pm C_{9}$ then, by Lemma 2.2.1, $G$ contains a subgroup $N$ isomorphic to $C_{18} \cdot C_{3}$ of index at most 2. But then $O_{3}(N)=O_{3}(G)$ has order 27. So we may assume that $F(G)=C_{9} \otimes H$ with $H \simeq C_{4}, D_{8}$ or $Q_{8}$. In any case $G$ contains an irreducible cyclic subgroup of order 36. Hence the result follows from Lemma 4.7.4.

Lemma 4.7.10 If $O_{3}(G)=C_{3}$, then $G$ is conjugate to one of $\sqrt{-3}\left[6 . U_{4}(3) .2\right]_{6}$, $\sqrt{-3}\left[C_{6}\right]_{1} \otimes M_{6,2}, \sqrt{-3}\left[ \pm 3 . M_{10}\right]_{6}, \sqrt{-15}\left[ \pm 3 . \operatorname{Alt}_{6} \cdot 2_{1}\right]_{6}, \sqrt{-15}\left[ \pm 3 . \mathrm{Alt}_{6}: 2_{1}\right]_{6}, \infty, 2\left[\mathrm{SL}_{2}(5)\right]_{3} \circ C_{3}$, $\sqrt{-7}\left[ \pm \mathrm{L}_{2}(7)\right]_{3} \underset{\sqrt{-7}}{\otimes} \infty\left[\tilde{S}_{3}\right]_{1}, \sqrt{-7}\left[ \pm \mathrm{L}_{2}(7)\right]_{3} \otimes A_{2}$.

Proof: By Table 2.5.1, $E(G)$ is either trivial, irreducible or isomorphic to $\mathrm{Alt}_{5}, \mathrm{~L}_{2}(7)$, $U_{4}(2)$. Let $N:=O_{3}(G) E(G)$. This leaves the following cases:

(a) If $E(G)=1$ then $G$ is soluble and $O_{2}(G) \leq C_{G}\left(O_{3}(G)\right)$ embeds into $\mathrm{GL}_{6}(\mathbb{Q}(\sqrt{-3}))$. Thus $O_{2}(G)$ is isomorphic to one of $C_{2}, C_{4}, D_{8}$ or $Q_{8}$. The normal subgroup $U:=\mathcal{B}^{\circ}\left(O_{2}(G)\right) O_{3}(G) \unlhd G$ is self centralizing and embeds into $\mathrm{GL}_{2}(\mathbb{Q}(\sqrt{-3}))$. But since $G / U \leq \operatorname{Out}(U)$ is a 2-group, this contradicts Corollary 2.2.3.

(b) If $E(G) \simeq$ Alt $_{5}$ then $C:=\operatorname{End}(\bar{N}) \simeq \mathbb{Q}(\sqrt{5}, \sqrt{-3})$ and denote by $K \simeq \mathbb{Q}(\sqrt{5})$ its maximal totally real subfield. Then $N$ fixes up to isomorphism 4 lattices whose endomorphism ring equals $\mathbb{Z}_{C}$. But only one of them, call it $L$, has minimal superlattices which have not this maximal order as endomorphism ring. So $L$ is $N$-normal critical since $C$ has class number 1 (see Remark 2.2.8). We find some $F \in \mathcal{F}_{>0}(N)$ such that $\operatorname{det}(L, F)=2^{4} \cdot 3^{6}$. Moreover $G / N \leq C_{2} \times C_{2}$ shows that $\Pi(|G|)=\{2,3,5\}$. By 2.5.4 $G$ is conjugate to $\operatorname{Aut}_{\mathbb{Q}(\sqrt{-d})}\left(L, \mathfrak{p}_{5}^{\alpha} F\right)$ for some $\alpha \in\{0,1\}$ and $d \in\{3,15\}$.

\begin{tabular}{|c|c|c|}
\hline form & $\mathbb{Q}(\sqrt{-3})$ & $\mathbb{Q}(\sqrt{-15})$ \\
\hline \hline$F$ & $\lesseqgtr \sqrt{-3}\left[C_{6}\right]_{1}^{6}$ & $\subsetneq \sqrt{-15}\left[ \pm 3 . \mathrm{Alt}_{6} \cdot 2_{1}\right]_{6}$ \\
\hline $\mathfrak{p}_{5} F$ & $\sqrt{-3}\left[C_{6}\right]_{1} \otimes M_{6,2}$ & $\subsetneq \sqrt{-15}\left[ \pm 3 . \mathrm{Alt}_{6}: 2_{1}\right]_{6}$ \\
\hline
\end{tabular}

So $G \simeq \sqrt{-3}\left[C_{6}\right]_{1} \otimes M_{6,2}$.

(c) If $E(G) \simeq \mathrm{L}_{2}(7)$ then $G$ contains an irreducible cyclic subgroup of order 21. Thus $G$ is conjugate to $\sqrt{-7}\left[ \pm \mathrm{L}_{2}(7)\right]_{3} \underset{\sqrt{-7}}{\otimes} \infty\left[\tilde{S}_{3}\right]_{1}$ or $\sqrt{-7}\left[ \pm \mathrm{L}_{2}(7)\right]_{3} \otimes A_{2}$ by Lemma 4.7.5.

(d) If $E(G) \simeq$ Alt $_{7}$ or $E(G) \simeq U_{4}(2)$ then $N$ is irreducible with commuting algebra $\mathbb{Q}(\sqrt{-3})$. One checks that they have only one s.i.m.f. supergroup which is $\sqrt{-3}\left[6 . U_{4}(3) .2\right]_{6}$. But this group does not contain $N$ as a normal subgroup.

(e) Finally if $E(G)$ is irreducible (and quasisimple), Lemma 4.7.6 shows that $G$ is conjugate to $\sqrt{-3}\left[6 . U_{4}(3) .2\right]_{6}, \sqrt{-3}\left[ \pm 3 . M_{10}\right]_{6}, \sqrt{-15}\left[ \pm 3 . \mathrm{Alt}_{6} \cdot 2_{1}\right]_{6}, \sqrt{-15}\left[ \pm 3 . \mathrm{Alt}_{6}: 2_{1}\right]_{6}$ or $\infty, 2\left[\mathrm{SL}_{2}(5)\right]_{3} \circ C_{3}$. 
Lemma 4.7.11 If $O_{p}(G)=1$ for all odd primes $p$, then $O_{2}(G)$ is conjugate to one of $C_{2}, C_{4}$ or $D_{8}$ and $E(G) \neq 1$.

Proof: If $\mathrm{O}_{2}(G)$ is not isomorphic to $C_{2}, C_{4}$ and $D_{8}$, then Corollary 2.1.16 and Table 2.5 .2 show that $O_{2}(G) \in \mathcal{O}:=\left\{Q_{8}, C_{8}, D_{16}, Q D_{16}, D_{8} \otimes C_{4}, 2_{+}^{1+4}\right\}$.

Suppose first $E(G)=1$. For any possible $O_{2}(G)$ it follows from loc. cit. that $G / \mathcal{B}^{\circ}\left(O_{2}(G)\right)$ is a 2-group and $\mathcal{B}^{\circ}\left(O_{2}(G)\right)$ embeds into $\mathrm{GL}_{k}(\mathbb{Q})$ for some $k \in\{1,2,4\}$. This contradicts Corollary 2.2.3. Hence $E(G) \neq 1$.

Finally, suppose $O_{2}(G) \in \mathcal{O}$. Then $\operatorname{End}\left(\overline{O_{2}(G)}\right) \simeq \mathrm{GL}_{3}(Q)$ where $Q$ is isomorphic to one of $\mathbb{Q}, \mathbb{Q}(i), \mathbb{Q}(\sqrt{ \pm 2})$ or $\mathcal{Q}_{\infty, 2}$. But then Table 2.5.1 implies $E(G)=1$ which is impossible (note that $\mathbb{Q}(\sqrt{5})$ and $\mathbb{Q}(\sqrt{-7})$ do not split $\left.\mathcal{Q}_{\infty, 2}\right)$.

Lemma 4.7.12 If $F(G)$ is conjugate to $C_{4}$ or $D_{8}$ then $G$ is conjugate to one of

$$
\begin{gathered}
\infty, 3\left[U_{3}(3)\right]_{3} \circ C_{4}, \underset{\sqrt{-5}}{ }\left[{ }_{i}\left[C_{4}\right]_{1} \stackrel{2_{+}}{\otimes} \mathrm{Alt}_{5}\right]_{6}, \sqrt{-5}\left[i\left[C_{4}\right]_{1} \stackrel{2-}{\otimes} \mathrm{Alt}_{5}\right]_{6},{ }_{i}\left[C_{4}\right]_{1} \otimes A_{6}^{(2)}, \\
{ }_{i}\left[\sqrt{-7}\left[ \pm \mathrm{L}_{2}(7)\right]_{3} \stackrel{2(2)}{\otimes}{ }_{i}\left[C_{4}\right]_{1}\right]_{6},{ }_{i}\left[\mathrm{~L}_{2}(7) \stackrel{2(2)}{\otimes}\left[C_{4}\right]_{1}\right]_{6},{ }_{i}\left[C_{4}\right]_{1} \otimes A_{6},{ }_{i}\left[C_{4}\right]_{1} \otimes E_{6}
\end{gathered}
$$

Proof: If $E(G)$ is irreducible, then $G \simeq{ }_{\infty, 3}\left[U_{3}(3)\right]_{3} \circ C_{4}$ according to Lemma 4.7.6. Suppose now that $E(G)$ is not irreducible. Then $E(G)$ embeds into $\mathrm{GL}_{6}(\mathbb{Q}(i))$ since $F(G)$ contains a characteristic subgroup $U$ isomorphic to $C_{4}$. Table 2.5.1 shows that there are five possibilities left.

(a) If $E(G) \simeq \operatorname{Alt}_{5}$ then $N:=\operatorname{Alt}_{5} \otimes C_{4} \unlhd G$. The commuting algebra of $N$ is isomorphic to $\mathbb{Q}(i, \sqrt{5})$ with $\mathbb{Q}(\sqrt{5})$ as maximal totally real subfield. Since $G / N \leq C_{2} \times C_{2}$ we have $\Pi(|G|)=\{2,3,5\}$. Up to isomorphism, $N$ fixes 3 lattices that have the maximal order as endomorphism ring. One of them is $N$-normal critical, $L$ say. One finds some $F \in \mathcal{F}_{>0}(N)$ that is integral on $L$ such that $\operatorname{det}(L, F)=1$. By Table 2.5.4 this leaves 4 candidates:

\begin{tabular}{|c|c|c|}
\hline form & $\mathbb{Q}(\sqrt{-5})$ & $\mathbb{Q}(i)$ \\
\hline \hline$F$ & $\sqrt{-5}\left[i\left[C_{4}\right]_{1} \stackrel{2_{-}}{\otimes} \mathrm{Alt}_{5}\right]_{6}$ & $\succ_{i}\left[C_{4}\right]_{1}^{6}$ \\
\hline $\mathfrak{p}_{5} F$ & $\sqrt{-5}\left[{ }_{i}\left[C_{4}\right]_{1} \stackrel{2_{+}}{\otimes} \mathrm{Alt}_{5}\right]_{6}$ & $\succ_{i}\left[C_{4}\right]_{1}^{6}$ \\
\hline
\end{tabular}

So $G \simeq \sqrt{-5}\left[{ }_{i}\left[C_{4}\right]_{1} \stackrel{2_{-}}{\otimes} \mathrm{Alt}_{5}\right]_{6}$ or $\sqrt{-5}\left[{ }_{i}\left[C_{4}\right]_{1} \stackrel{2_{+}}{\otimes} \mathrm{Alt}_{5}\right]_{6}$.

(b) If $E(G)$ is conjugate to ${ }_{\sqrt{-7}}\left[\mathrm{~L}_{2}(7)\right]_{3}$ then $N:={ }_{\sqrt{-7}}\left[ \pm \mathrm{L}_{2}(7)\right]_{3} \otimes C_{4} \unlhd G$. The commuting algebra $C$ of $N$ is isomorphic to $\mathbb{Q}(i, \sqrt{-7})$. Let $K \simeq \mathbb{Q}(\sqrt{7})$ be its maximal totally real subfield. Since $G / N \leq \operatorname{Out}(N) \simeq C_{2} \times C_{2}$, we have $\Pi(|G|)=\{2,3,7\}$. The group $N$ fixes only one lattice $L$ and there exists some $F \in \mathcal{F}_{>0}(H)$ that is integral on $L$ such that $\operatorname{det}(L, F)=7^{6} . \operatorname{Since}_{\operatorname{End}_{\mathbb{Z} N}}(L)$ is the maximal order in $C$ and since $\mathbb{Z}_{K,>0}^{*}=\operatorname{Nr}_{C / K}\left(\mathbb{Z}_{C}^{*}\right)$ we may restrict ourselves to one class of totally positive units. Let $\langle\sigma\rangle=\operatorname{Gal}(C / K) \simeq C_{2}$. One finds that $\sigma$ is conjugation by some $x \in N_{\mathrm{GL}_{12}(\mathbb{Q})}(N) \cap \mathrm{GL}(L)$ with $x F x^{\operatorname{tr}}=F$. So $x$ 
interchanges the two prime ideals over 3 (and necessarily fixes the unique prime ideals over 2 and 7). By Table 2.5.4 this leaves the following 8 automorphism groups:

\begin{tabular}{|c|c|c|}
\hline form & $\mathbb{Q}(i)$ & $\mathbb{Q}(\sqrt{-7})$ \\
\hline$F$ & ${ }_{i}\left[C_{4}\right]_{1} \otimes A_{6}^{(2)}$ & $\bar{~} \overline{\sqrt{-7}}\left[ \pm \mathrm{L}_{2}(7)\right]_{3}^{2}$ \\
\hline $\mathfrak{p}_{2} F$ & $i\left[\sqrt{-7}\left[ \pm \mathrm{L}_{2}(7)\right]_{3} \stackrel{2(2)}{\otimes}_{i}\left[C_{4}\right]_{1}\right]_{6}$ & $\lesseqgtr \sqrt{-7}\left[ \pm \mathrm{L}_{2}(7)\right]_{3}^{2}$ \\
\hline $\mathfrak{p}_{3} \mathfrak{p}_{7}^{-1} F$ & ${ }_{\infty, 3}\left[U_{3}(3)\right]_{4} \circ C_{4}$ & $\sqrt{-7}\left[ \pm \mathrm{L}_{2}(7)\right]_{3} \underset{\sqrt{-7}}{\otimes} \infty, 3\left[\tilde{S}_{3}\right]$ \\
\hline $\mathfrak{p}_{2} \mathfrak{p}_{3} \mathfrak{p}_{7}^{-1} F$ & $N$ & $N$ \\
\hline
\end{tabular}

So $G \simeq{ }_{i}\left[C_{4}\right]_{1} \otimes A_{6}^{(2)}$ or ${ }_{i}\left[\sqrt{-7}\left[ \pm \mathrm{L}_{2}(7)\right]_{3} \stackrel{2(2)}{\otimes}{ }_{i}\left[C_{4}\right]_{1}\right]_{6}$.

(c) If $E(G)$ is conjugate to $\mathrm{L}_{2}(7)$ with character $2 \chi_{6}$ then $N:=\mathrm{L}_{2}(7) \otimes C_{4} \unlhd G$ fixes up to isomorphism 6 lattices and its commuting algebra is isomorphic to $\mathbb{Q}(i)$. One checks that $N$ is only contained in ${ }_{i}\left[C_{4}\right]_{1} \otimes A_{6}$ or ${ }_{i}\left[\mathrm{~L}_{2}(7)^{2(2)} \otimes_{i}\left[C_{4}\right]_{1}\right]_{6}$. So $G \simeq{ }_{i}\left[\mathrm{~L}_{2}(7) \stackrel{2(2)}{\otimes} \otimes_{i}\left[C_{4}\right]_{1}\right]_{6}$.

(d) If $E(G) \simeq \operatorname{Alt}_{7}$ then $\mathcal{B}^{o}\left(F^{*}(G)\right) \simeq{ }_{i}\left[C_{4}\right]_{1} \otimes A_{6}$ is already s.i.m.f..

(e) If $E(G) \simeq U_{4}(2)$ then $\mathcal{B}^{o}\left(F^{*}(G)\right) \simeq{ }_{i}\left[C_{4}\right]_{1} \otimes E_{6}$ is already s.i.m.f..

Lemma 4.7.13 If $F(G) \simeq C_{2}$ then $G$ is conjugate to ${ }_{\sqrt{-2}}\left[ \pm \mathrm{L}_{2}(7) \cdot 2\right]_{6}, \sqrt{-11}\left[\mathrm{SL}_{2}(11)\right]_{6}$ or $\sqrt{-2}\left[\infty, 2\left[\mathrm{SL}_{2}(5)\right]_{3}: 2\right]_{6}$.

Proof: If $E(G)$ is irreducible, then Lemma 4.7.6 shows that $G \simeq \sqrt{-11}\left[\mathrm{SL}_{2}(11)\right]_{6}$. Otherwise Table 2.5.1 shows that $E(G)$ is isomorphic to $A_{1 t}, A_{1}, U_{4}(2)$ or $\mathrm{L}_{2}(7)$ (with two representations). The 6-dimensional representation of $\mathrm{Alt}_{5}$ extends to $\pm \mathrm{Alt}_{5}: 2$ in $\mathrm{GL}_{6}(\mathbb{Q})$ by $\left[\mathrm{CCN}^{+} 85\right]$. The element $\frac{1+\sqrt{5}}{2} \in \mathbb{Q}(\sqrt{5}) \simeq C_{\mathbb{Q}^{6 \times 6}}\left(\mathrm{Alt}_{5}\right)$ has norm -1 . Thus also $\pm \mathrm{Alt}_{5} \cdot 2<\mathrm{GL}_{6}(\mathbb{Q})$. So $E(G) \not \mathrm{Alt}_{5}$. If $E(G) \simeq \mathrm{Alt}_{7}$ or $U_{4}(2)$ then $G / \pm E(G) \leq \operatorname{Out}(E(G)) \simeq C_{2} \simeq \mathcal{B}^{\circ}(E(G)) / \pm E(G)$ shows that $G=\mathcal{B}^{\circ}(E(G))$ is reducible. Suppose $E(G)$ is conjugate to $\mathrm{L}_{2}(7)$ with character $2 \chi_{3 a b}$. Then $G / \pm E(G) \leq \operatorname{Out}(E(G)) \simeq C_{2}$ contradicts Lemma 2.2.1. Finally suppose $E(G)$ is conjugate to $\mathrm{L}_{2}(7)$ with character $2 \chi_{6}$. The extension $\pm \mathrm{L}_{2}(7): 2$ embeds into $\mathrm{GL}_{6}(\mathbb{Q}(\sqrt{2}))$ (see $\left.\left[\mathrm{CCN}^{+} 85\right]\right)$. The split extension $\sqrt{-2}\left[ \pm \mathrm{L}_{2}(7) \cdot 2\right]_{6}<\mathrm{GL}_{6}(\mathbb{Q}(\sqrt{-2}))$ is maximal finite as one easily verifies. 


\subsection{Dimension 14}

Theorem 4.8.1 The s.i.m.f. subgroups $G$ of $\operatorname{Sp}_{14}(\mathbb{Q})$ are

\begin{tabular}{|c|c|c|c|c|c|}
\hline$\#$ & $G$ & $|G|$ & $|\mathcal{Z}(G)|$ & $L_{\text {min }}$ & r.i.m.f. supergroups \\
\hline \hline$[2,1,7]$ & ${ }_{i}\left[C_{4}\right]_{1}^{7}$ & $2^{18} \cdot 3^{2} \cdot 5 \cdot 7$ & 3 & {$[1,1,28]$} & $B_{14}$ \\
\hline 1 & ${ }_{i}\left[C_{4}\right]_{1} \otimes E_{7}$ & $2^{11} \cdot 3^{4} \cdot 5 \cdot 7$ & 3 & {$[1,2,252]$} & $E_{7}^{2}$ \\
\hline 2 & ${ }_{i}\left[U_{3}(3) \circ C_{4}\right]_{7}$ & $2^{7} \cdot 3^{3} \cdot 7$ & 4 & {$\left[2^{6} \cdot 3^{2}, 3,112\right]$} & {$\left[U_{3}(3) \stackrel{\circ}{2} C_{4}\right]_{14}$} \\
\hline \hline$[2,2,7]$ & $\sqrt{-3}\left[C_{6}\right]_{1}^{7}$ & $2^{11} \cdot 3^{9} \cdot 5 \cdot 7$ & 1 & {$\left[3^{7}, 2,42\right]$} & $A_{2}^{7}$ \\
\hline 3 & $\sqrt{-3}\left[C_{6}\right]_{1} \otimes E_{7}$ & $2^{10} \cdot 3^{5} \cdot 5 \cdot 7$ & 2 & {$\left[2^{2} \cdot 3^{7}, 4,378\right]$} & $A_{2} \otimes E_{7}$ \\
\hline
\end{tabular}

Proof: By explicit calculations, one verifies that the above table is correct and yields s.i.m.f. groups. Further the r.i.m.f. supergroups are easily constructed since all s.i.m.f. groups are uniform. It remains to show the completeness of the classification. The group $\sqrt{-3}\left[C_{6}\right]_{1}^{7}$ is s.i.m.f. by Lemma 2.1.21. The group ${ }_{i}\left[C_{4}\right]_{1}^{7}$ fixes 3 lattices and has $\mathbb{Q}(i)$ as commuting algebra. One verifies that it is s.i.m.f.. So it remains to prove that the completeness of the s.p.i.m.f. matrix groups.

Let $G<\operatorname{Sp}_{14}(\mathbb{Q})$ be s.p.i.m.f.. Then Corollary 4.1 .2 shows that $E(G) \neq 1$. Thus $N:=\mathcal{B}^{o}(E(G))$ is conjugate to $A_{7}, E_{7}$ or $U_{3}(3) \circ C_{4}$ by Table 2.5.1.

The group ${ }_{i}\left[U_{3}(3) \circ C_{4}\right]_{7}$ is already s.i.m.f.. In the other two cases, $\operatorname{Out}(N)$ is trivial. Thus by Lemma 4.1.1, $G$ contains a subgroup conjugate to $C_{k} \otimes N$ with $k \in\{4,6\}$. If $N \simeq E_{7}$, this yields the maximal finite subgroups stated above. The groups $\sqrt{-3}\left[C_{6}\right]_{1} \otimes A_{7}$ and ${ }_{i}\left[C_{4}\right]_{1} \otimes A_{7}$ are irreducible and fix 4 and 7 lattices respectively. One checks that they are only contained in ${ }_{\sqrt{-3}}\left[C_{6}\right]_{1} \otimes E_{7}$ and ${ }_{i}\left[C_{4}\right]_{1} \otimes E_{7}$ respectively. 


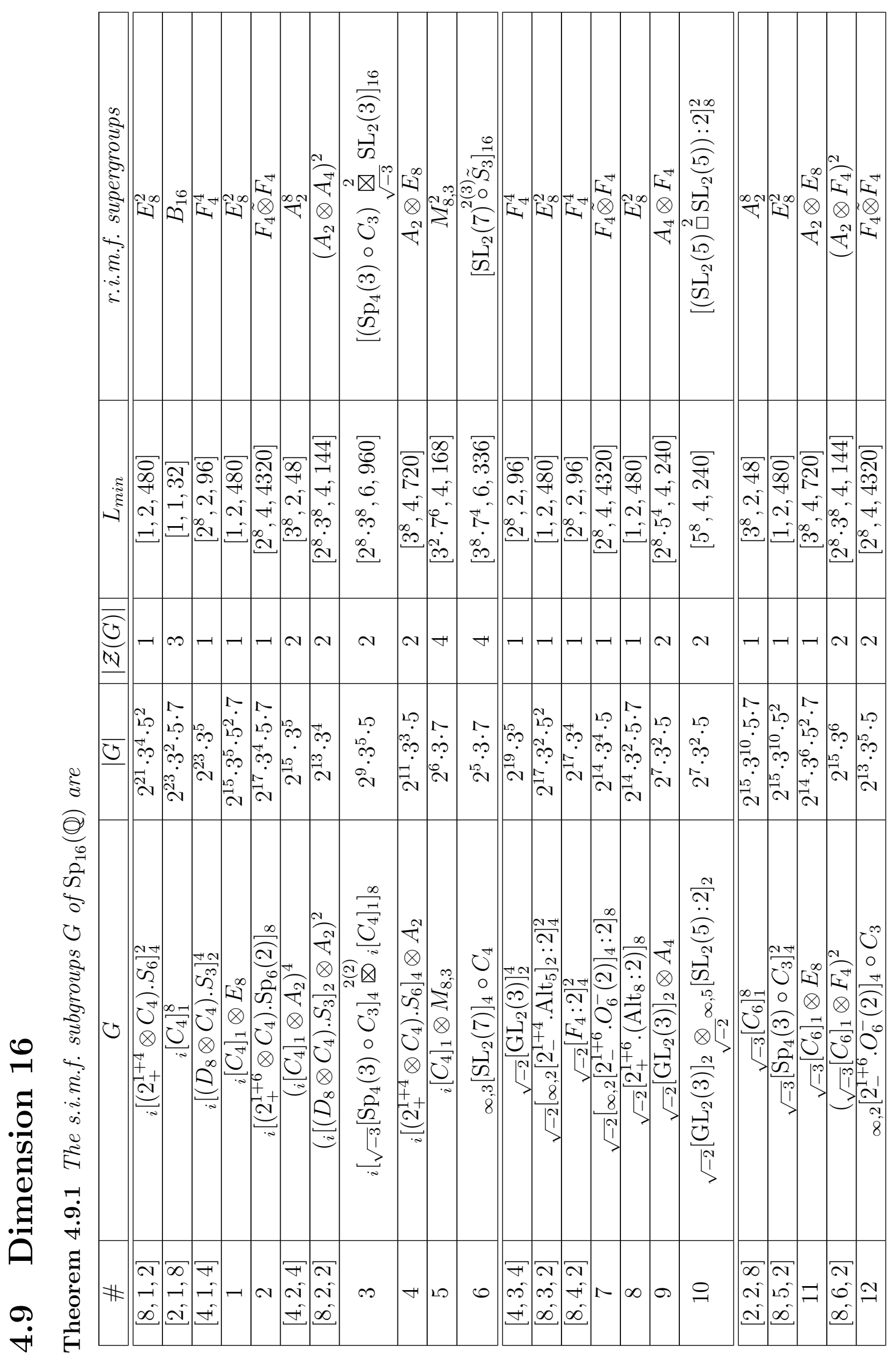




\begin{tabular}{|c|c|c|c|c|c|c|c|c|c|c|c|c|c|c|c|c|c|c|}
\hline 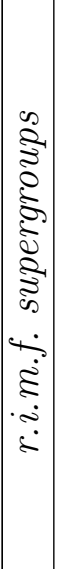 & $\mid$ & 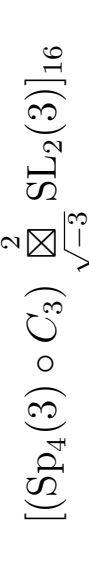 & 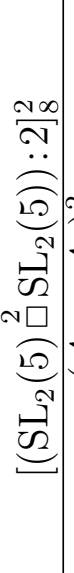 & 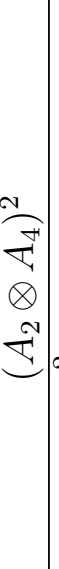 & 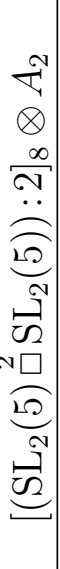 & $\begin{array}{l}\vec{T}_{+}^{+} \\
\otimes \\
\vec{\square}\end{array}$ & 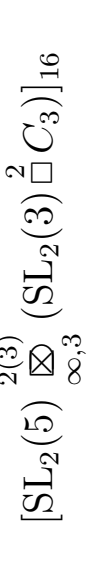 & 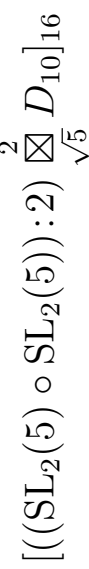 & 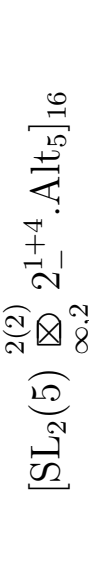 & 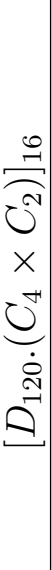 & $\underset{[\infty}{\infty}]^{\infty}$ & 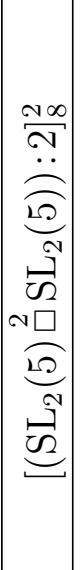 & No & 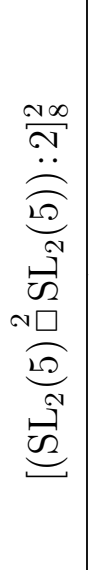 & $\stackrel{+}{\longrightarrow}$ & $\begin{array}{l}I_{1}^{+1} \\
i \otimes x^{+1} \\
I^{+1}\end{array}$ & 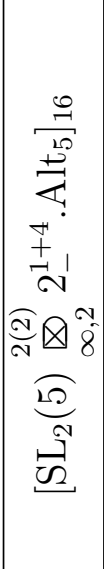 & 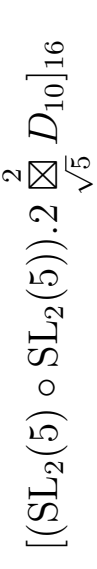 \\
\hline $\mid$ & 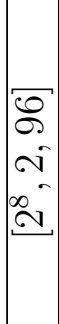 & 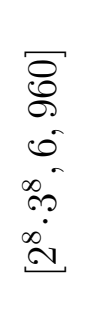 & 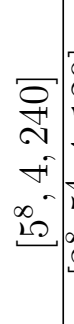 & 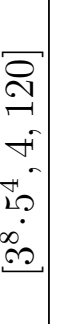 & $\begin{array}{l} \\
8 \\
\infty \\
\infty \\
\infty \\
\infty \\
\infty \\
i \\
\infty \\
\infty \\
\infty\end{array}$ & 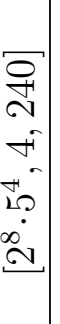 & 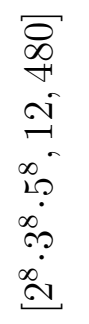 & 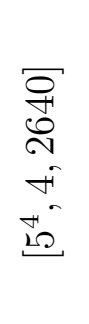 & 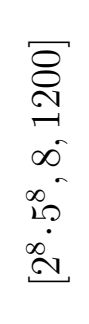 & 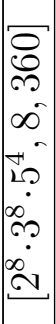 & 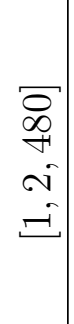 & 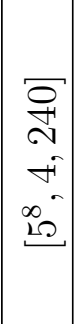 & 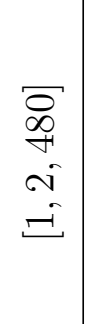 & $\begin{array}{l}\stackrel{0}{1} \\
\stackrel{\sim}{1} \\
+ \\
\infty \\
0 \\
0\end{array}$ & \begin{tabular}{|l|}
$\infty$ \\
$\infty$ \\
$\sim$ \\
$\vdots$ \\
1 \\
10 \\
\end{tabular} & 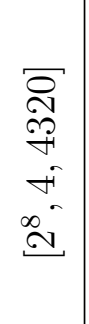 & 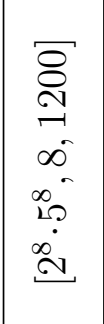 & 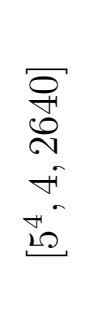 \\
\hline 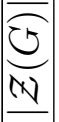 & $\curvearrowright$ & N & $N$ & $N$ & $N$ & $\nabla$ & $\forall$ & $\nabla$ & $\nabla$ & $\infty$ & $N$ & $\sim$ & $N$ & $N$ & $\forall$ & $\sim$ & $N$ & $\forall$ \\
\hline$\circlearrowright$ & 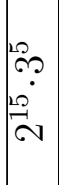 & $\begin{array}{l}\stackrel{20}{0} \\
\ddot{2} \\
\ddot{2} \\
\ddot{2}\end{array}$ & 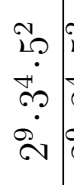 & 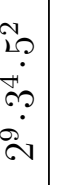 & 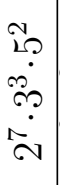 & 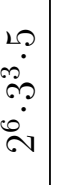 & $\begin{array}{l}\stackrel{2}{0} \\
0 \\
\because \\
0 \\
0\end{array}$ & $\begin{array}{l}\text { है? } \\
\text { คै? } \\
\text { ?ैं }\end{array}$ & 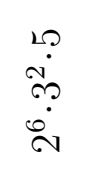 & $\mid \begin{array}{l}20 \\
\ddot{2} \\
\ddot{2} \\
\stackrel{2}{2}\end{array}$ & 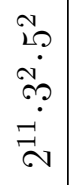 & 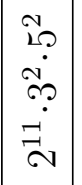 & 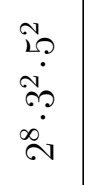 & $\begin{array}{l}\tilde{i} \\
i \\
0 \\
\infty \\
\infty\end{array}$ & 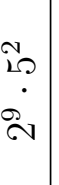 & 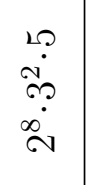 & 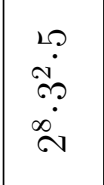 & 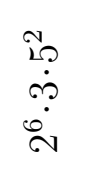 \\
\hline$\circlearrowleft$ & 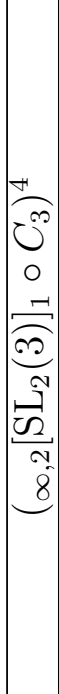 & 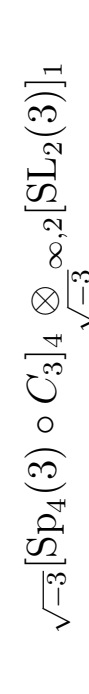 & 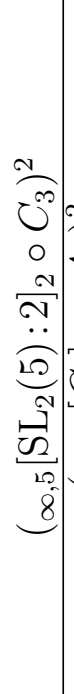 & 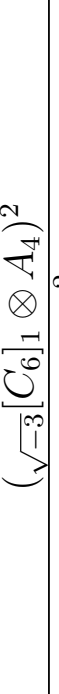 & 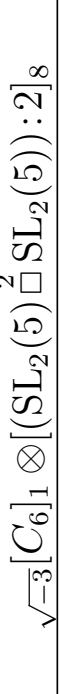 & 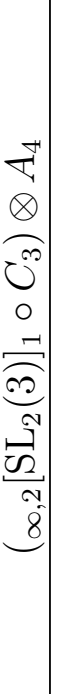 & 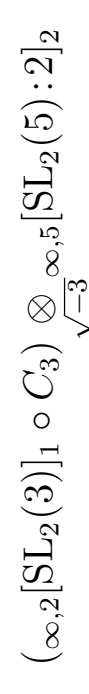 & 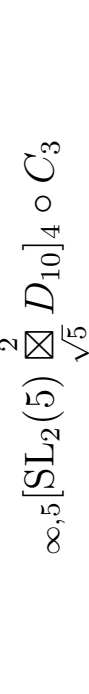 & 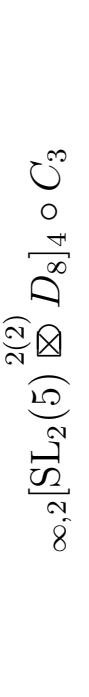 & 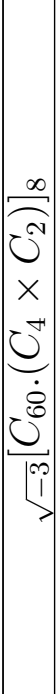 & 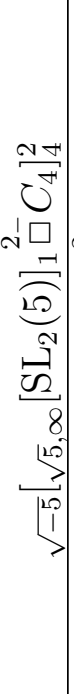 & 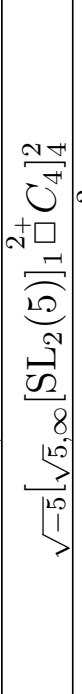 & 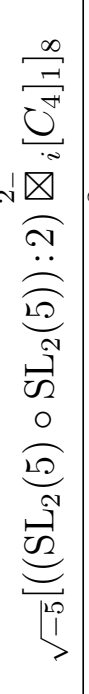 & 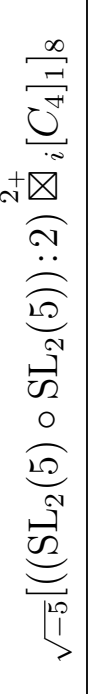 & 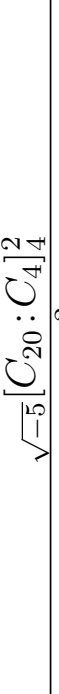 & 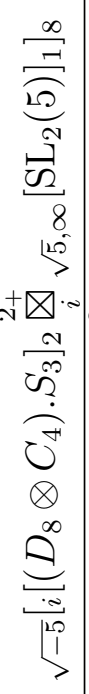 & 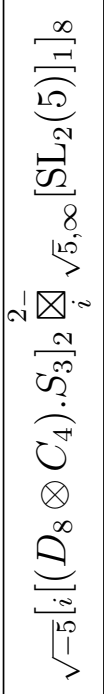 & 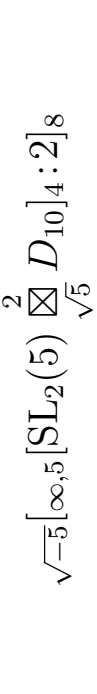 \\
\hline \# & $\begin{array}{l}\vec{f} \\
+ \\
+ \\
+1\end{array}$ & $\stackrel{m}{\rightarrow}$ & $\begin{array}{l}\mathbf{N} \\
N \\
\infty \\
\infty\end{array}$ & $\begin{array}{l}N \\
\infty \\
\infty \\
\infty\end{array}$ & $\nexists$ & $\stackrel{10}{10}$ & 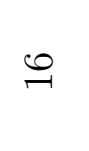 & 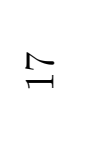 & 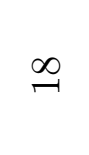 & $\stackrel{\Omega}{-1}$ & $\begin{array}{l}\tilde{N} \\
\tilde{\sigma} \\
\infty \\
\infty\end{array}$ & $\begin{array}{c}\bar{N} \\
0 \\
- \\
\infty \\
\infty\end{array}$ & $\stackrel{\sim}{\sim}$ & $\vec{N}$ & {$\left[\begin{array}{l}N \\
- \\
= \\
\infty \\
\infty\end{array}\right]$} & $\stackrel{\sim}{N}$ & $\stackrel{6}{4}$ & $\vec{N}$ \\
\hline
\end{tabular}




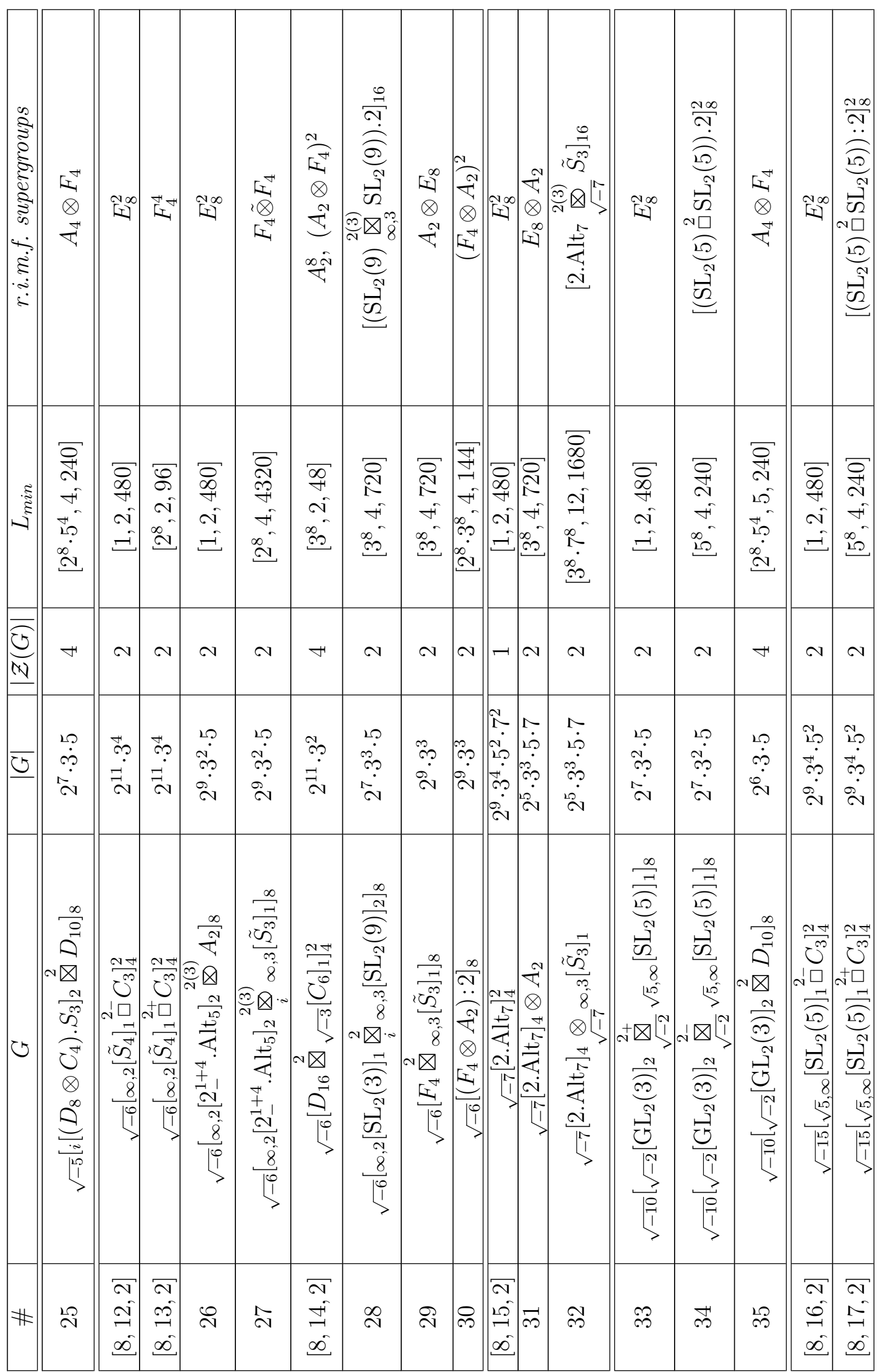




\begin{tabular}{|c|c|c|c|c|c|c|c|c|c|c|c|c|c|c|c|c|}
\hline $\begin{array}{l}\text { है } \\
\text { है } \\
\text { है } \\
\text { है }\end{array}$ & $\begin{array}{l}\underset{+\infty}{\infty} \\
\underset{\nabla}{+}\end{array}$ & 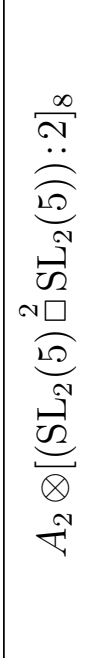 & 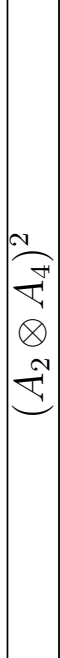 & 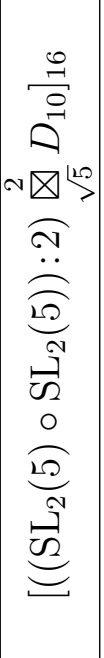 & 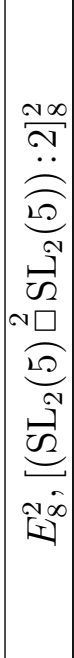 & $\mid$ & 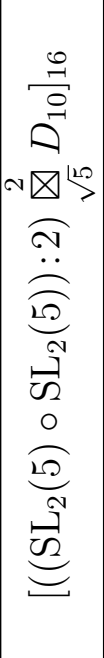 & 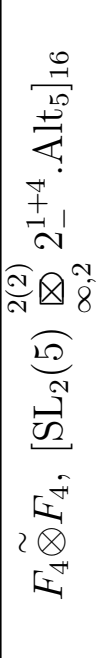 & 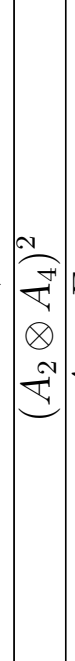 & 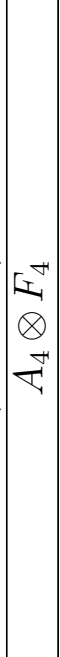 & 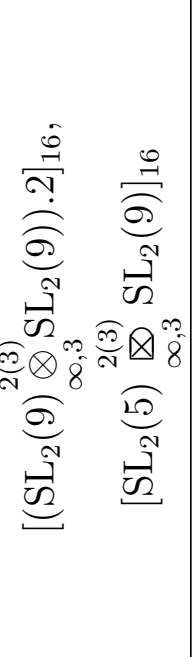 & 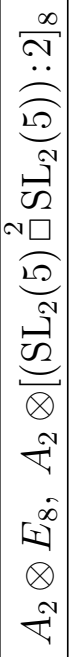 & 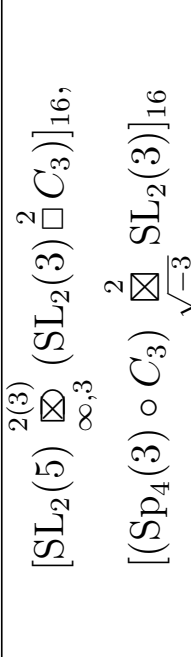 & 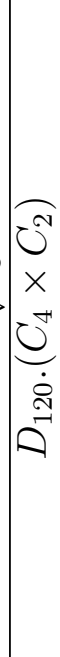 & 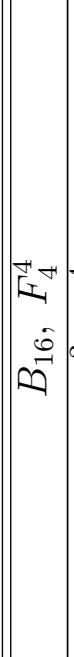 & 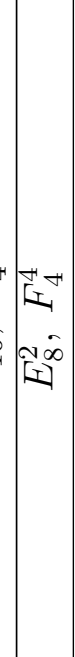 \\
\hline |- & 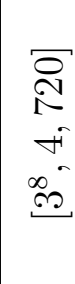 & $\begin{array}{l}\text { ?̊ } \\
0 \\
\infty \\
\infty \\
\infty \\
10 \\
\infty \\
\infty \\
0\end{array}$ & 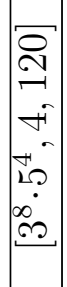 & 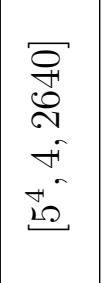 & 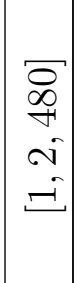 & 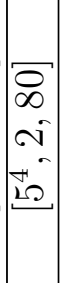 & 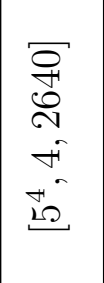 & 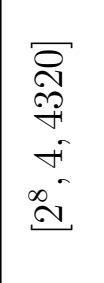 & 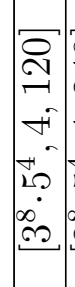 & 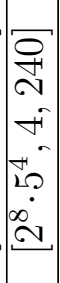 & 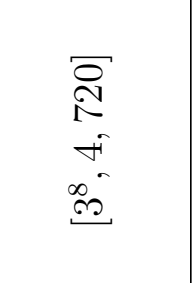 & 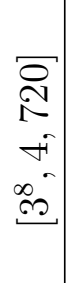 & 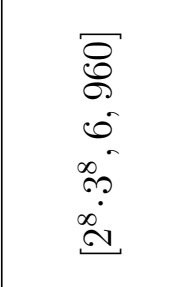 & 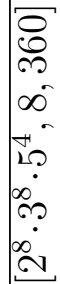 & 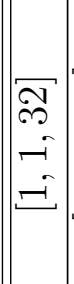 & $\mid \begin{array}{c}\infty \\
\infty \\
+ \\
\sim \\
\sim \\
\sim\end{array}$ \\
\hline$\frac{\circlearrowright}{C N}$ & $\sim$ & $N$ & $\nabla$ & 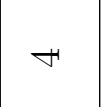 & -1 & -1 & -1 & N & $\sim$ & N & $N$ & 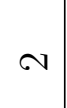 & 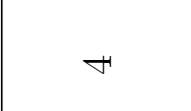 & $F$ & $F$ & $\curvearrowright$ \\
\hline Ð & 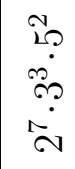 & 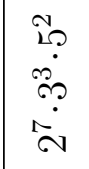 & 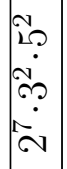 & 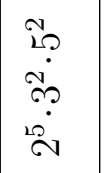 & 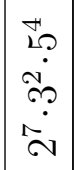 & 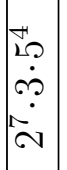 & 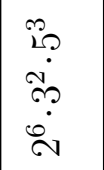 & 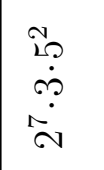 & 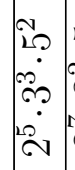 & 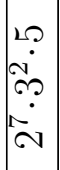 & 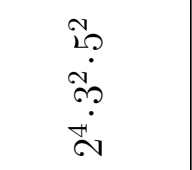 & 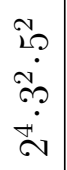 & 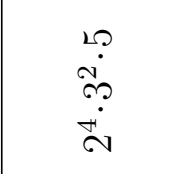 & 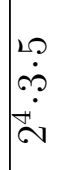 & $\vec{\nabla}$ & $\begin{array}{l}\sim \\
\infty \\
\sim\end{array}$ \\
\hline ひ & 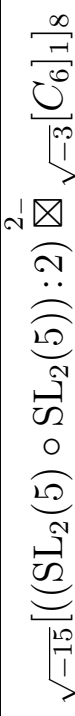 & 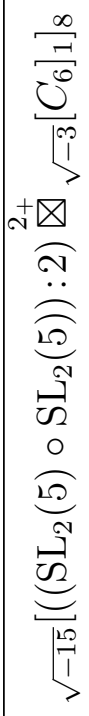 & 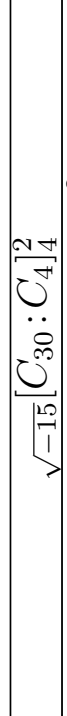 & 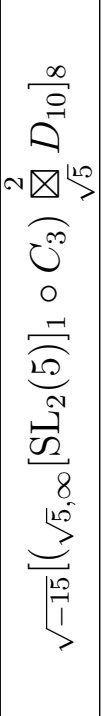 & 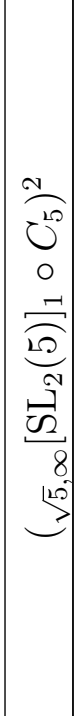 & 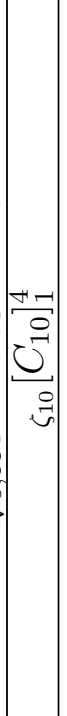 & 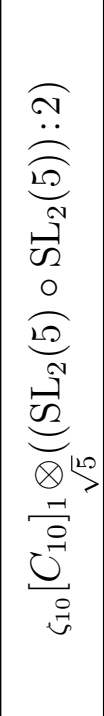 & 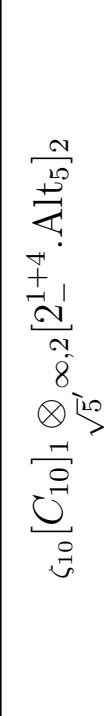 & 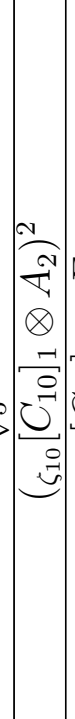 & 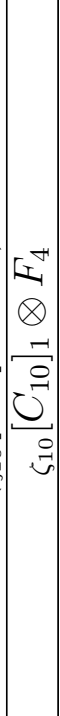 & 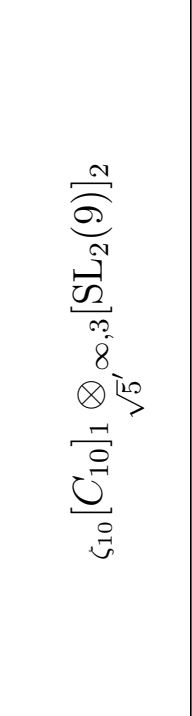 & 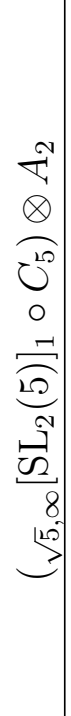 & 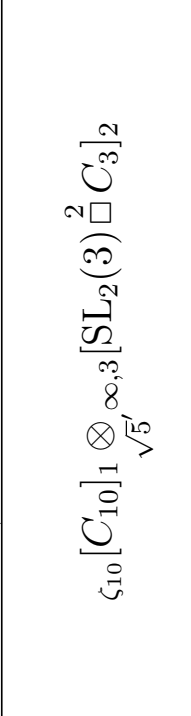 & 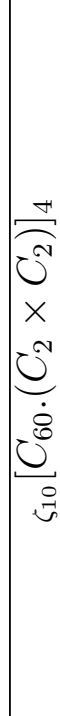 & 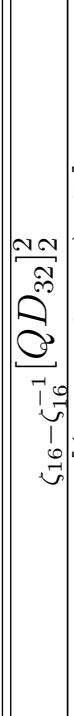 & 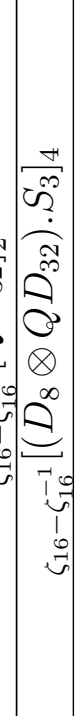 \\
\hline \# & m & mo & $\begin{array}{l}\sim \\
\infty \\
\infty \\
-1 \\
\infty\end{array}$ & $\infty$ & $\begin{array}{l}\vec{N} \\
\infty \\
\infty \\
\infty\end{array}$ & $\mid \begin{array}{l}F \\
20 \\
\forall \\
\end{array}$ & m & 아 & $\begin{array}{c}\tilde{N} \\
\tilde{N} \\
\tilde{N} \\
\infty\end{array}$ & $F$ & $\stackrel{\text { I }}{4}$ & $\stackrel{9}{\rightarrow}$ & $F$ & $\stackrel{10}{+}$ & $\begin{array}{l}\vec{N} \\
\tilde{N} \\
\infty \\
\infty\end{array}$ & $\stackrel{0}{q}$ \\
\hline
\end{tabular}




\begin{tabular}{|c|c|c|c|c|c|c|c|c|c|c|c|c|c|c|c|c|}
\hline \multicolumn{2}{|r|}{ 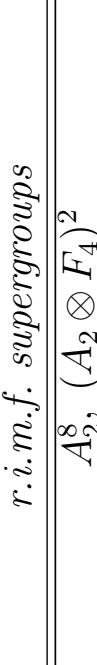 } & 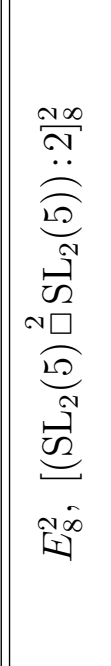 & 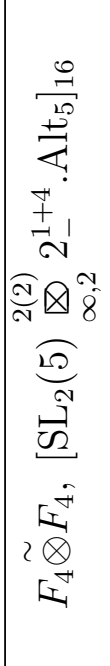 & 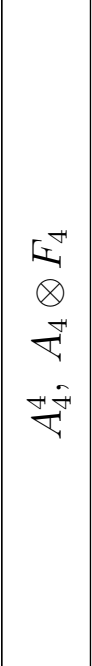 & 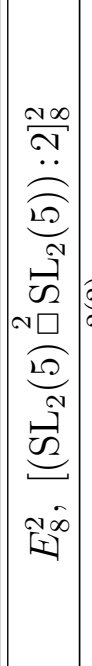 & 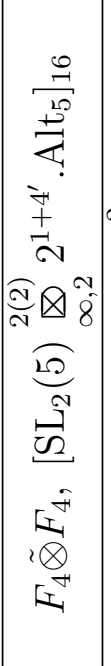 & 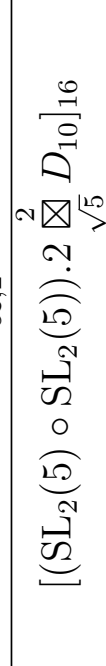 & 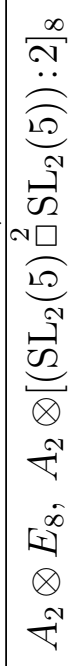 & 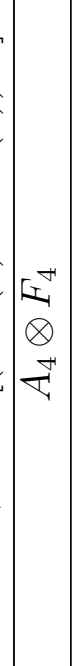 & 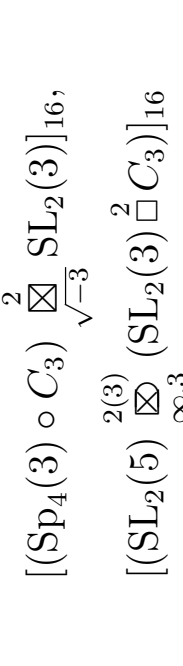 & 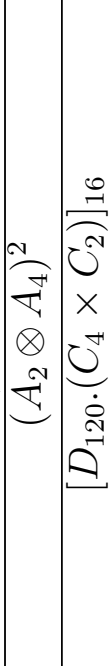 & 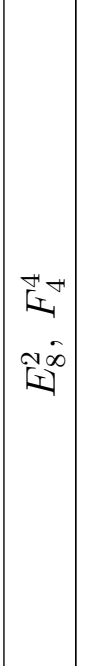 & 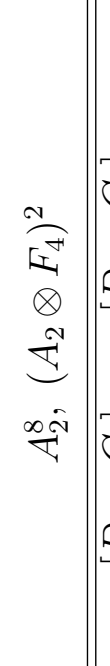 & 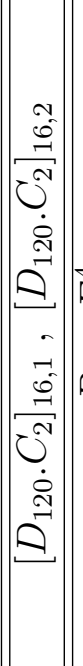 & $\mid$ & 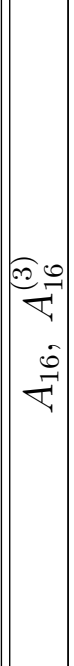 \\
\hline & 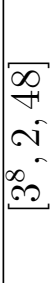 & 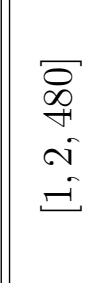 & 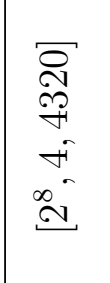 & $\begin{array}{c}\bar{\infty} \\
0 \\
i \\
i 0 \\
i 0\end{array}$ & 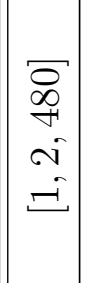 & 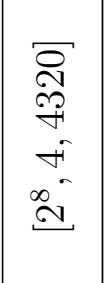 & 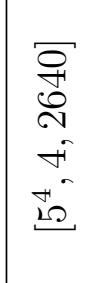 & 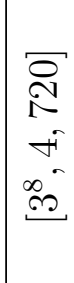 & 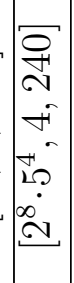 & 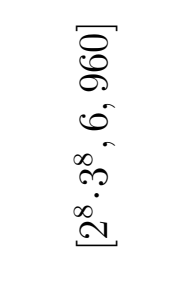 & 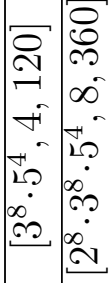 & 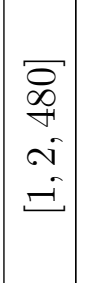 & $\begin{array}{l}\infty \\
\infty \\
0 \\
\infty \\
\infty \\
0 \\
0\end{array}$ & 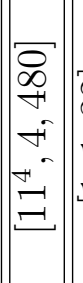 & 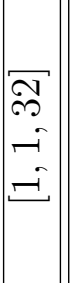 & $\mid \begin{array}{ll} & \mathbb{N} \\
N \\
N \\
N \\
N \\
\\
\end{array}$ \\
\hline & r & $N$ & N & $F^{\prime}$ & $F$ & $F$ & F & 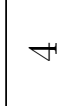 & $\ngtr$ & $F$ & $\Rightarrow r$ & $\forall$ & $H$ & F & $\sim$ & -1 \\
\hline & $\stackrel{?}{\stackrel{9}{*}}$ & 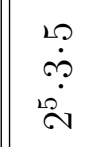 & $\begin{array}{l}\stackrel{2}{0} \\
\ddot{2} \\
\stackrel{0}{2}\end{array}$ & $\begin{array}{l}10 \\
\stackrel{2}{2}\end{array}$ & $\begin{array}{l}L \\
\ddot{0} \\
\stackrel{1}{N}\end{array}$ & 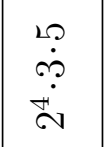 & 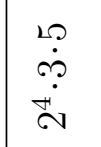 & 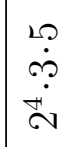 & & $\begin{array}{l}\stackrel{0}{\circ} \\
\stackrel{\leftrightarrow}{N} \\
\stackrel{N}{N}\end{array}$ & 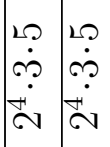 & $\stackrel{?}{\stackrel{2}{0}}$ & $\begin{array}{l}\Re \\
\stackrel{\circ}{0}\end{array}$ & 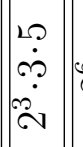 & $\stackrel{\circ}{\circ}$ & $\mid \begin{array}{l}\vec{\sim} \\
\dot{\sim}\end{array}$ \\
\hline & 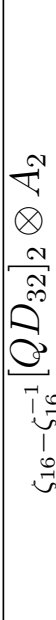 & 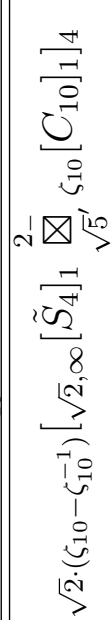 & 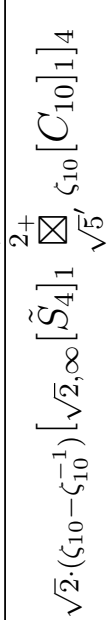 & 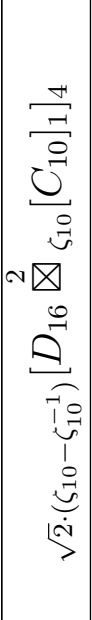 & 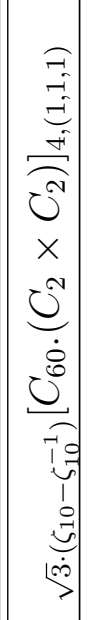 & 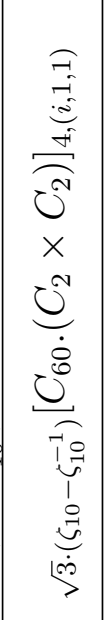 & 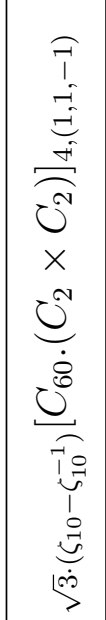 & 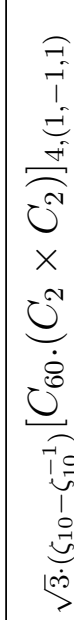 & 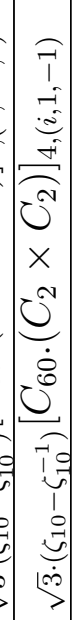 & 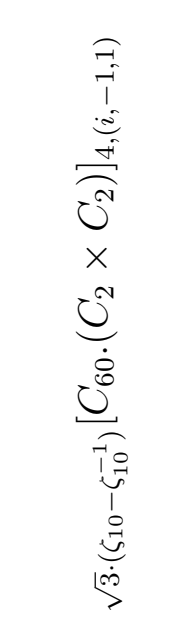 & 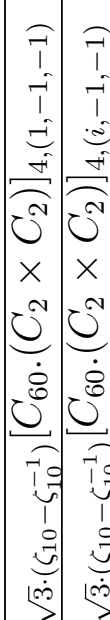 & 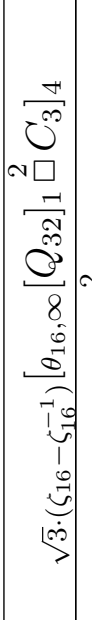 & 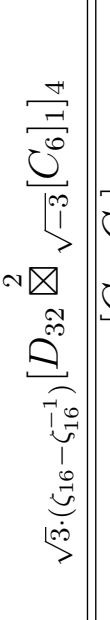 & 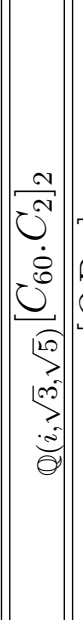 & 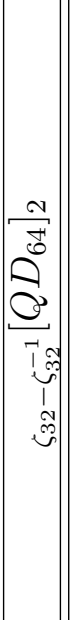 & $\frac{-7}{0^{0}}$ \\
\hline & tr & $\stackrel{\infty}{+\infty}$ & $\stackrel{F}{F}$ & 88 & 20 & î & i⿱ & $\overrightarrow{b 0}$ & 20 & 象 & {$\left[\begin{array}{l}1 \\
15\end{array}\right.$} & 象 & 8 & $\overrightarrow{0}$ & ชิ & (8ి \\
\hline
\end{tabular}


The eleven s.p.i.m.f. subgroups $G<\operatorname{Sp}_{16}(\mathbb{Q})$ with an irreducible normal cyclic subgroup of order 60 have the following presentations:

- $\sqrt{-3}\left[C_{60} \cdot\left(C_{4} \times C_{2}\right)\right]_{4} \simeq\left\langle x, \alpha, \beta \mid x^{60}, \alpha^{4}, \beta^{2}, x^{\alpha}=x^{7}, x^{\beta}=x^{19}, \alpha^{\beta}=x^{15} \alpha\right\rangle$

- ${ }_{\zeta_{10}}\left[C_{60} \cdot\left(C_{2} \times C_{2}\right)\right]_{4} \simeq\left\langle x, \alpha, \beta \mid x^{60}, \alpha^{2}, \beta^{2}, x^{\alpha}=x^{11}, x^{\beta}=x^{31}, \alpha^{\beta}=x^{15} \alpha\right\rangle$

- $\mathbb{Q}(i, \sqrt{3}, \sqrt{5})\left[C_{60} \cdot C_{2}\right]_{2} \simeq\left\langle x, \alpha \mid x^{60}, \alpha^{2}=x^{15}, x^{\alpha}=x^{49}\right\rangle$.

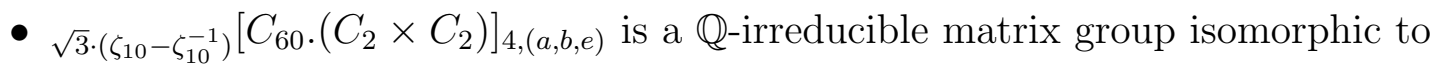

$$
\left\langle x, \alpha, \beta \mid x^{60}, x^{\alpha}=-x^{-1}, x^{\beta}=x^{19}, \alpha^{2}=a, \beta^{2}=b, \alpha \beta=e \beta \alpha^{-1}\right\rangle
$$

for $e, b \in\{ \pm 1\}$ and $a \in\left\{1, i:=x^{15}\right\}$.

To distinguish between the matrix groups $\sqrt{-5}\left[i\left[\left(D_{8} \otimes C_{4}\right) . S_{3}\right]_{2} \underset{i}{2_{+}} \underset{\sqrt{5}, \infty}{ }\left[\mathrm{SL}_{2}(5)\right]_{1}\right]_{8}$ and $\sqrt{-5}\left[i\left[\left(D_{8} \otimes C_{4}\right) \cdot S_{3}\right]_{2} \underset{i}{2_{-}} \underset{5, \infty}{ }\left[\mathrm{SL}_{2}(5)\right]_{1}\right]_{8}$ we make the following convention. These two groups are generated by ${ }_{i}\left[\left(D_{8} \otimes C_{4}\right) \cdot S_{3}\right]_{2} \otimes_{i}{ }_{\sqrt{5}, \infty}\left[\mathrm{SL}_{2}(5)\right]_{1}$ and some $\alpha$ that centralizes the unique normal subgroup isomorphic to $Q_{8}$ in $O_{2}(G) \simeq D_{8} \otimes C_{4} \simeq Q_{8} \circ C_{4}$. Up to conjugacy one has the two choices $\alpha^{2}=I_{16}$ and $\alpha^{2}=-I_{16}$. In the first case we write $\underset{i}{2_{+}}$and in the second case we use $\underset{i}{\mathbb{2}_{-}}$.

Similarly, the two s.p.i.m.f. matrix groups $\sqrt{-10}\left[\sqrt{-2}\left[\mathrm{GL}_{2}(3)\right]_{2} \underset{\sqrt{-2}}{\stackrel{2_{+}}{\otimes}} \sqrt{5}, \infty\left[\mathrm{SL}_{2}(5)\right]_{1}\right]_{8}$ and $\sqrt{-10}\left[\sqrt{-2}\left[\mathrm{GL}_{2}(3)\right]_{2} \underset{\sqrt{-2}}{\stackrel{2}{\otimes}} \sqrt{5, \infty}\left[\mathrm{SL}_{2}(5)\right]_{1}\right]_{8}$ are generated by $\sqrt{-2}\left[\mathrm{GL}_{2}(3)\right]_{2} \underset{\sqrt{-2}}{\otimes} \sqrt{5}, \infty\left[\mathrm{SL}_{2}(5)\right]_{1}$ and some $\alpha$ that centralizes $\mathcal{B}^{\circ}\left(O_{2}(G)\right) \simeq{ }_{\infty, 2}\left[\mathrm{SL}_{2}(3)\right]_{1}$. Again there are up to conjugacy two possibilities. We write $\underset{\sqrt{-2}}{2_{+}}$for $\alpha^{2}=I_{16}$ and $\underset{\sqrt{-2}}{\stackrel{2}{\otimes}}$ means $\alpha^{2}=-I_{16}$.

Lemma 4.9.2 All groups listed in Theorem 4.9.1 are s.i.m.f..

Proof: The candidates for the maximal finite symplectic imprimitive matrix groups come from the classification of the s.p.i.m.f. subgroups of $\operatorname{Sp}_{2 m}(\mathbb{Q})$ with $m \in\{1,2,4\}$. All groups except ${ }_{i}\left[C_{4}\right]_{1}^{8}$ and ${ }_{\zeta_{16}-\zeta_{16}^{-1}}\left[Q D_{32}\right]_{2}^{2}$ are s.i.m.f. by Lemma 2.1.21. These two groups have $\mathbb{Q}(i)$ and $\mathbb{Q}\left(\zeta_{16}-\zeta_{16}^{-1}\right)$ as commuting algebras and they fix up to isomorphism 3 and 4 lattices respectively. One checks that they are maximal finite by computing the corresponding automorphism groups (wrt. the full form space).

So we may now assume that $G<\operatorname{Sp}_{16}(\mathbb{Q})$ is s.p.i.m.f.. According to Theorem 3.1.1, $\zeta_{34}\left[C_{34}\right]_{1}$ is the unique s.i.m.f. subgroup of $\operatorname{Sp}_{16}(\mathbb{Q})$ whose order is a multiple of 17 . If $G$ is any other group from the above table whose order is not divisible by 17 and which is not isomorphic to $C_{60} \cdot C_{2}$, then $E:=\operatorname{End}(\bar{G})$ is a minimal totally complex field. Thus these groups are checked to be s.i.m.f. by computing $\operatorname{Aut}_{E}(L, F)$ where $F \in$ $\mathcal{F}_{>0}(G)$ is fixed and $L$ runs through a system of representatives for the isomorphism classes of $\mathcal{Z}(G)$. Obviously, $G$ is not a subgroup of $\left[D_{120} \cdot C_{2}\right]_{16, i}$ see ([NP95, (IV.1) 
Theorem]). Thus loc. cit. shows that any r.i.m.f. supergroup of $G$ fixes a primitive lattice of determinant only divisible by $2,3,5$ or 7 . So the r.i.m.f. supergroups can be constructed by the $m$-parameter argument.

Finally, suppose that $G={ }_{\mathbb{Q}(i, \sqrt{3}, \sqrt{5})}\left[C_{60} \cdot C_{2}\right]_{2}$. Let $C:=\operatorname{End}(\bar{G}) \simeq \mathbb{Q}(i, \sqrt{3}, \sqrt{5})$ and denote by $K \simeq \mathbb{Q}(\sqrt{3}, \sqrt{5})$ its maximal totally real subfield. Since $C$ is not minimal totally complex, the $m$-parameter argument would be quite tedious. We give another proof of the maximality, using only the classification of the r.i.m.f. subgroups of $\mathrm{GL}_{16}(\mathbb{Q})$ (see [NP95, (IV.1) Theorem]).

The group $G$ fixes up to isomorphism four lattices $L_{1}, \ldots, L_{4}$ which can be chosen such that the index $\left[L_{1}: L_{i}\right]$ is a power of 5. Further one finds some $F \in \mathcal{F}_{>0}(G)$ that is integral on $L_{1}$ with $\operatorname{det}\left(L_{1}, F\right)=11^{4}$.

Suppose $H$ is a r.i.m.f. supergroup of $G$. Let $\left(L^{\prime}, F^{\prime}\right) \in \mathcal{Z}(G) \times \mathcal{F}_{>0}(G)$ be integral and of minimal determinant among all such integral pairs. Let $d=\operatorname{det}\left(L^{\prime}, F^{\prime}\right)$. The classification of the r.i.m.f. subgroups of $\mathrm{GL}_{16}(\mathbb{Q})$ shows that $\Pi(d) \subseteq\{2,3,5,7,11\}$. There exist $1 \leq i \leq 4, c \in C$ and $x \in K_{>0}$ such that $L^{\prime}=L_{i} c$ and $F^{\prime}=x F$. Then $\left(L^{\prime}, F^{\prime}\right)$ is isometric to $\left(L_{i}, x \mathrm{Nr}_{C / K}\left(c^{-1}\right) F\right)$. If we set $a:=x \mathrm{Nr}_{C / K}\left(c^{-1}\right) \in K_{>0}$ then $d=\operatorname{det}\left(L_{i}, F\right) \cdot \mathrm{Nr}_{K / \mathbb{Q}}(a)^{4}=5^{k} \cdot 11^{4} \cdot \mathrm{Nr}_{K / \mathbb{Q}}(a)^{4}$ for some $k \in \mathbb{Z}$. Since the prime ideals of $\mathbb{Z}_{K}$ over 2,3,5 and 7 are generated by Elements in $K_{>0}$ and the ideals over 11 are not, this implies that $d$ is divisible by 11 . The classification of the r.i.m.f. subgroups of $\mathrm{GL}_{16}(\mathbb{Q})$ shows that $H$ can only be conjugate to $\left[D_{120} \cdot C_{2}\right]_{16,1}$ or $\left[D_{120} \cdot C_{2}\right]_{16,2}$. One immediately constructs $G$ as a subgroup of these groups. In any case $[H: G]=2$ and $H$ is not symplectic. This implies that $G$ is s.i.m.f. and we have found its r.i.m.f. supergroups.

It remains to prove that Theorem 4.9.1 contains every conjugacy class of s.p.i.m.f. matrix groups. This is accomplished in the remainder of this section. As always, we first classify the s.i.m.f. groups that contain an irreduble cyclic subgroup. Afterwards, we turn to a case by case discussion of the various Fitting subgroups.

But before we start, we find all s.p.i.m.f. groups that contain some irreducible normal subgroup $N$ where $\operatorname{End}(\bar{N})$ is a quaternion algebra.

Lemma 4.9.3 If $G<\operatorname{Sp}_{16}(\mathbb{Q})$ is s.p.i.m.f. and contains a normal subgroup $N$ conjugate to $\infty_{\infty, 3}\left[\mathrm{SL}_{2}(7)\right]_{4}$, then $G$ is conjugate to $\infty, 3\left[\mathrm{SL}_{2}(7)\right]_{4} \circ C_{4}$.

Proof: The commuting algebra of $N$ is isomorphic to $\mathcal{Q}_{\infty, 3}$ and $\operatorname{Out}(N) \simeq C_{2}$. Hence by Remark 2.2.17, $G$ contains a subgroup conjugate to $N \circ C_{4}, N \circ C_{3}$ or $N .2$. The first group is maximal finite. The other two groups fix 4 and 16 lattices respectively. One checks that they both have only one s.i.m.f. supergroup, which is $\sqrt{-3}\left[C_{6}\right]_{1}^{8}$.

Lemma 4.9.4 If $G<\operatorname{Sp}_{16}(\mathbb{Q})$ is s.p.i.m.f. and contains a normal subgroup $N \simeq 2_{-}^{1+6}$, then $G$ is conjugate to $\sqrt{-2}\left[\infty, 2\left[2_{-}^{1+6} . O_{6}^{-}(2)\right]_{4}: 2\right]_{8}$ or ${ }_{\infty, 2}\left[2_{-}^{1+6} . O_{6}^{-}(2)\right]_{4} \circ C_{3}$.

Proof: The normal subgroup $B:=\mathcal{B}^{\circ}(N) \simeq{ }_{\infty, 2}\left[2_{-}^{1+6} \cdot O_{6}^{-}(2)\right]_{4}$ of $G$ has $\mathcal{Q}_{\infty, 2}$ as commuting algebra and $\operatorname{Out}(B) \simeq C_{2}$. Hence by Remark 2.2.17, $G$ contains a subgroup conjugate to $B \circ C_{4}, B \circ C_{3}$ or $B .2$. Their commuting algebras are isomorphic to $\mathbb{Q}(i), \mathbb{Q}(\sqrt{-3})$ and $\mathbb{Q}(\sqrt{-2})$ respectively. Since they only fix 1 or 2 lattices, it is easily verified that $B \circ C_{4}$ is only contained in ${ }_{i}\left[\left(2_{+}^{1+6} \otimes C_{4}\right) \cdot \operatorname{Sp}_{6}(2)\right]_{8}$ whereas the other two groups are s.i.m.f.. 


\subsubsection{Irreducible cyclic subgroups}

In this section, we classify all s.i.m.f. groups $G<\operatorname{Sp}_{16}(\mathbb{Q})$ that contain an irreducible cyclic subgroup $U$ (under some assumptions on $\Pi(|G|)$ ). There are essentially 5 such groups $U$ since $| \pm U| \in\left\{k \in \mathbb{N} \mid k \equiv_{2} 0\right.$ and $\left.\varphi(k)=16\right\}=\{32,34,40,48,60\}$.

The group $\zeta_{34}\left[C_{34}\right]_{1}$ is the unique symplectic irreducible group $G$ whose order is divisible by 17 as we have seen in Theorem 3.1.1.

Theorem 4.9.5 If $|U|=32$ then $G$ is conjugate to $\zeta_{16}-\zeta_{16}^{-1}\left[Q D_{32}\right]_{2}^{2}, \zeta_{32}-\zeta_{32}^{-1}\left[Q D_{64}\right]_{2}$, $\sqrt{-2}\left[\mathrm{GL}_{2}(3)\right]_{2}^{4},{ }_{i}\left[C_{4}\right]_{1}^{8}$ or ${ }_{i}\left[\left(D_{8} \otimes C_{4}\right) \cdot S_{3}\right]_{2}^{4}$.

Proof: By Minkowski's bound and Theorem 3.1.1 we have $\Pi(|G|) \subseteq\{2,3,5,7,11,13\}$. The group $U$ fixes only one lattice $L$ since $\operatorname{End}(U) \simeq \mathbb{Q}\left(\zeta_{32}\right)$ has class number 1 . Further there exists some $F \in \mathcal{F}_{>0}(U)$ that is integral on $L$ with $\operatorname{det}(L, F)=1$. Let $\sigma \in \operatorname{Gal}\left(\mathbb{Q}\left(\theta_{32}\right) / \mathbb{Q}\right)$ such that $\sigma$ interchanges the two prime ideals over 7. One finds that $\sigma$ is conjugation by some $x \in N_{\mathrm{GL}_{16}(\mathbb{Q})}(U) \cap \mathrm{GL}(L)$. Hence by Table 2.5.3, $G$ must be conjugate to $\operatorname{Aut}_{K}\left(L, \mathfrak{p}_{2}^{a} \mathfrak{p}_{7}^{b} F\right)$ for some $0 \leq b \leq 4, a \in\{0,1\}$ and $K \in\left\{\mathbb{Q}(i), \mathbb{Q}(\sqrt{-2}), \mathbb{Q}\left(\zeta_{16}-\zeta_{16}^{-1}\right), \mathbb{Q}\left(\zeta_{32}-\zeta_{32}^{-1}\right)\right\}$

If $K=\mathbb{Q}\left(\zeta_{16}-\zeta_{16}^{-1}\right)$ or $\mathbb{Q}\left(\zeta_{32}-\zeta_{32}^{-1}\right)$ then $G$ is conjugate to $\zeta_{16}-\zeta_{16}^{-1}\left[Q D_{32}\right]_{2}^{2}$ or $\zeta_{32}-\zeta_{32}^{-1}\left[Q D_{64}\right]_{2}$ respectively. Finally, $\operatorname{Aut}_{\mathbb{Q}(\sqrt{-2})}\left(L, \mathfrak{p}_{2}^{k} \mathfrak{p}_{7}^{l} F\right) \leq \sqrt{-2}\left[\mathrm{GL}_{2}(3)\right]_{2}^{4}$ and $\operatorname{Aut}_{\mathbb{Q}(i)}\left(L, \mathfrak{p}_{2}^{a} \mathfrak{p}_{7}^{b} F\right) \leq\left\{\begin{array}{ll}{ }_{i}\left[C_{4}\right]_{1}^{8} & \text { if } 0 \leq b \leq 2 \\ i\left[\left(D_{8} \otimes C_{4}\right) . S_{3}\right]_{2}^{4} & \text { if } b \in\{3,4\}\end{array}\right.$.

Theorem 4.9.6 Suppose $|U|=40$ and $\Pi(|G|)=\{2,3,5\}$ or there exists some $(L, F) \in \mathcal{Z}(G) \times \mathcal{F}_{>0}(G)$ such that $F$ is integral on $L$ and $\Pi(\operatorname{det}(L, F)) \subseteq\{2,3,5\}$.

Then $G$ is conjugate to one of

$$
\begin{aligned}
& { }_{i}\left[\left(2_{+}^{1+4} \otimes C_{4}\right) \cdot S_{6}\right]_{4}^{2},{ }_{i}\left[\left(2_{+}^{1+6} \otimes C_{4}\right) \cdot \operatorname{Sp}_{6}(2)\right]_{8},{ }_{i}\left[{ }_{\sqrt{-3}}\left[\operatorname{Sp}_{4}(3) \circ C_{3}\right]_{4} \stackrel{2(2)}{\otimes}{ }_{i}\left[C_{4}\right]_{1}\right]_{8}, \\
& \sqrt{-2}\left[2_{+}^{1+6} \cdot\left(\operatorname{Alt}_{8}: 2\right)\right]_{8}, \sqrt{-2}\left[\infty, 2\left[2_{-}^{1+6} \cdot O_{6}^{-}(2)\right]_{4}: 2\right]_{8}, \sqrt{-2}\left[\mathrm{GL}_{2}(3)\right]_{2} \otimes A_{4}, \\
& \sqrt{-2}\left[\mathrm{GL}_{2}(3)\right]_{2} \underset{\sqrt{-2}}{\otimes} \infty, 5\left[\mathrm{SL}_{2}(5): 2\right]_{2}, \sqrt{-5}\left[\sqrt{5}, \infty\left[\mathrm{SL}_{2}(5)\right]_{1}^{2+}{ }^{2+} C_{4}\right]_{4}^{2}, \sqrt{-5}\left[\sqrt{5}, \infty\left[\mathrm{SL}_{2}(5)\right]_{1}^{2-} C_{4}\right]_{4}^{2} \text {, } \\
& \sqrt{-5}\left[C_{20}: C_{4}\right]_{4}^{2}, \sqrt{-5}\left[i\left[\left(D_{8} \otimes C_{4}\right) \cdot S_{3}\right]_{2} \stackrel{2}{\otimes} D_{10}\right]_{8}, \sqrt{-5}\left[i\left[\left(D_{8} \otimes C_{4}\right) \cdot S_{3}\right]_{2} \underset{i}{2_{+}} \underset{\sqrt{5}, \infty}{ }\left[\mathrm{SL}_{2}(5)\right]_{1}\right]_{8}, \\
& \sqrt{-5}\left[i\left[\left(D_{8} \otimes C_{4}\right) \cdot S_{3}\right]_{2} \underset{i}{2_{-}} \sqrt{5}, \infty\left[\mathrm{SL}_{2}(5)\right]_{1}\right]_{8}, \sqrt{-10}\left[\sqrt{-2}\left[\mathrm{GL}_{2}(3)\right]_{2} \underset{\sqrt{2}}{\stackrel{2_{+}}{\otimes}} \sqrt{5, \infty}\left[\mathrm{SL}_{2}(5)\right]_{1}\right]_{8}, \\
& \sqrt{-10}\left[\sqrt{-2}\left[\mathrm{GL}_{2}(3)\right]_{2} \underset{\sqrt{2}}{\stackrel{2}{\otimes}} \sqrt{5}, \infty\left[\mathrm{SL}_{2}(5)\right]_{1}\right]_{8}, \sqrt{-10}\left[\sqrt{-2}\left[\mathrm{GL}_{2}(3)\right]_{2} \stackrel{2}{\otimes} D_{10}\right]_{8},
\end{aligned}
$$

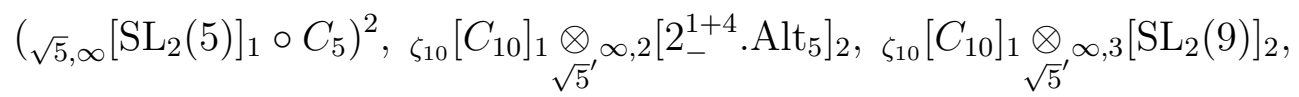

$$
\begin{aligned}
& \zeta_{10}\left[C_{10}\right]_{1} \underset{\sqrt{5}}{\otimes}, \infty, 3\left[\mathrm{SL}_{2}(3) \stackrel{2}{\square} C_{3}\right]_{2}, \quad \zeta_{10}\left[C_{10}\right]_{1}^{4}, \quad \zeta_{10}\left[C_{10}\right]_{1} \otimes F_{4}, \quad \zeta_{10}\left[C_{60} \cdot\left(C_{2} \times C_{2}\right)\right]_{4},
\end{aligned}
$$

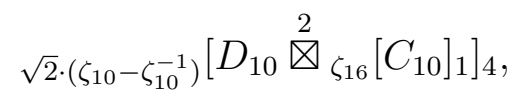

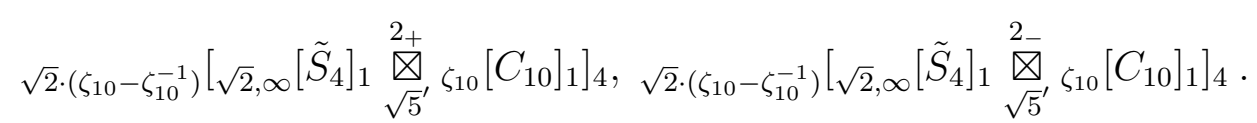


Proof: The commuting algebra $\operatorname{End}(\bar{U}) \simeq \mathbb{Q}\left(\zeta_{40}\right)$ has class number 1 . Thus $U$ fixes only one lattice $L$. Further there exists some $F \in \mathcal{F}_{>0}(U)$ that is integral on $L$ with $\operatorname{det}(L, F)=1$. Let $\sigma \in \operatorname{Gal}\left(\mathbb{Q}\left(\theta_{40}\right) / \mathbb{Q}\right)$ such that $\sigma$ interchanges the two prime ideals over 3. One finds that $\sigma$ is conjugation by some $x \in N_{\mathrm{GL}_{16}(\mathbb{Q})}(N) \cap \mathrm{GL}(L)$. Thus by Table 2.5.3. $G$ must be conjugate to $\operatorname{Aut}_{K}\left(L, \mathfrak{p}_{2}^{2 a} \mathfrak{p}_{3}^{b} \mathfrak{p}_{5}^{c} F\right)$ for some $a, b \in\{0,1\}$, $0 \leq c \leq 2$ and $K \in\left\{\mathbb{Q}(i), \mathbb{Q}(\sqrt{-2}), \mathbb{Q}(\sqrt{-5}), \mathbb{Q}(\sqrt{-10}), \mathbb{Q}\left(\zeta_{10}\right), \mathbb{Q}\left(\sqrt{2} \cdot\left(\zeta_{10}-\zeta_{10}^{-1}\right)\right)\right\}$.

\begin{tabular}{|c|c|c|}
\hline form & $\mathbb{Q}(i)$ & $\mathbb{Q}(\sqrt{-2})$ \\
\hline$F$ & ${ }_{i}\left[\left(2_{+}^{1+4} \otimes C_{4}\right) \cdot S_{6}\right]_{4}^{2}$ & $\sqrt{-2}\left[\left(2_{+}^{1+6} \cdot\left(\mathrm{Alt}_{8}: 2\right)\right]_{8}\right.$ \\
\hline $\mathfrak{p}_{2}^{2} F$ & ${ }_{i}\left[\left(2_{-}^{1+6} \otimes C_{4}\right) \cdot \operatorname{Sp}_{6}(2)\right]_{8}$ & $\sqrt{-2}\left[\infty, 2\left[2_{-}^{1+6} \cdot O_{6}^{-}(2)\right]_{4}: 2\right]_{8}$ \\
\hline $\mathfrak{p}_{3} F$ & $\lesseqgtr_{i}\left[\sqrt{-3}\left[\operatorname{Sp}_{4}(3) \circ C_{3}\right]_{4} \stackrel{2(2)}{\otimes}{ }_{i}\left[C_{4}\right]_{1}\right]_{8}$ & $\lesseqgtr \sqrt{-2}\left[\left(2_{+}^{1+6} \cdot\left(\mathrm{Alt}_{8}: 2\right)\right]_{8}\right.$ \\
\hline $\mathfrak{p}_{2}^{2} \mathfrak{p}_{3} F$ & $i\left[\sqrt{-3}\left[\operatorname{Sp}_{4}(3) \circ C_{3}\right]_{4} \stackrel{2(2)}{\otimes}_{i}\left[C_{4}\right]_{1}\right]_{8}$ & $\lesseqgtr \sqrt{-2}\left[\infty, 2\left[2_{-}^{1+6} \cdot O_{6}^{-}(2)\right]_{4}: 2\right]_{8}$ \\
\hline $\mathfrak{p}_{5} F$ & $\lesseqgtr_{i}\left[\left(2_{+}^{1+4} \otimes C_{4}\right) \cdot S_{6}\right]_{4}^{2}$ & $\lesseqgtr \sqrt{-2}\left[\mathrm{GL}_{2}(3)\right]_{2} \otimes A_{4}$ \\
\hline $\mathfrak{p}_{2}^{2} \mathfrak{p}_{5} F$ & $\Varangle_{i}\left[\left(2_{-}^{1+6} \otimes C_{4}\right) \cdot \operatorname{Sp}_{6}(2)\right]_{8}$ & $\sqrt{-2}\left[\mathrm{GL}_{2}(3)\right]_{2} \otimes A_{4}$ \\
\hline $\mathfrak{p}_{3} \mathfrak{p}_{5} F$ & $\lesseqgtr_{i}\left[\left(2_{+}^{1+4} \otimes C_{4}\right) \cdot S_{6}\right]_{4}^{2}$ & $\underbrace{}_{-2}\left[\mathrm{GL}_{2}(3)\right]_{2} \otimes A_{4}$ \\
\hline $\mathfrak{p}_{2}^{2} \mathfrak{p}_{3} \mathfrak{p}_{5} F$ & $\lesseqgtr_{i}\left[\sqrt{-3}\left[\operatorname{Sp}_{4}(3) \circ C_{3}\right]_{4} \stackrel{2(2)}{\otimes}_{i}\left[C_{4}\right]_{1}\right]_{8}$ & $\lesseqgtr \sqrt{-5}\left[i\left[\left(D_{8} \otimes C_{4}\right) \cdot S_{3}\right]_{2} \stackrel{2}{\otimes} D_{10}\right]_{8}$ \\
\hline $\mathfrak{p}_{5}^{2} F$ & $\lesseqgtr_{i}\left[\left(2_{+}^{1+4} \otimes C_{4}\right) \cdot S_{6}\right]_{4}^{2}$ & $\sqrt{-2}\left[\mathrm{GL}_{2}(3)\right]_{2} \underset{\sqrt{-2}}{\otimes} \infty, 5\left[\mathrm{SL}_{2}(5): 2\right]_{2}$ \\
\hline $\mathfrak{p}_{2}^{2} \mathfrak{p}_{5}^{2} F$ & $\succ_{i}\left[\left(2_{-}^{1+6} \otimes C_{4}\right) \cdot \operatorname{Sp}_{6}(2)\right]_{8}$ & $\lesseqgtr \sqrt{-2}\left[\mathrm{GL}_{2}(3)\right]_{2} \underset{\sqrt{-2}}{\otimes} \infty, 5\left[\mathrm{SL}_{2}(5): 2\right]_{2}$ \\
\hline $\mathfrak{p}_{3} \mathfrak{p}_{5}^{2} F$ & $\lesseqgtr_{i}\left[\left(2_{+}^{1+4} \otimes C_{4}\right) \cdot S_{6}\right]_{4}^{2}$ & $\sqrt{-2}\left[2_{-}^{1+6} \cdot\left(\text { Alt }_{8}: 2\right)\right]_{8}$ \\
\hline $\mathfrak{p}_{2}^{2} \mathfrak{p}_{3} \mathfrak{p}_{5}^{2} F$ & $\succ_{i}\left[\sqrt{-3}\left[\operatorname{Sp}_{4}(3) \circ C_{3}\right]_{4} \stackrel{2(2)}{\otimes}{ }_{i}\left[C_{4}\right]_{1}\right]_{8}$ & $\underbrace{}_{-5}\left[i\left[\left(D_{8} \otimes C_{4}\right) \cdot S_{3}\right]_{2} \stackrel{2}{\otimes} D_{10}\right]_{8}$ \\
\hline
\end{tabular}

\begin{tabular}{|c|c|c|}
\hline form & $\mathbb{Q}(\sqrt{-5})$ & $\mathbb{Q}\left(\zeta_{10}\right)$ \\
\hline$F$ & $\sqrt{-5}\left[\sqrt{5}, \infty\left[\mathrm{SL}_{2}(5)\right]_{1}^{2-} \square C_{4}\right]_{4}^{2}$ & $\left(\sqrt{5}, \infty\left[\mathrm{SL}_{2}(5)\right]_{1} \circ C_{5}\right)^{2}$ \\
\hline $\mathfrak{p}_{2}^{2} F$ & $\sqrt{-5}\left[i\left[\left(D_{8} \otimes C_{4}\right) \cdot S_{3}\right]_{2} \underset{i}{2_{+}} \underset{\sqrt{5}, \infty}{ }\left[\mathrm{SL}_{2}(5)\right]_{1}\right]_{8}$ & $\zeta_{10}\left[C_{10}\right]_{1} \underset{\sqrt{5}}{\otimes} \infty, 2\left[2_{-}^{1+4} \cdot \mathrm{Alt}_{5}\right]_{2}$ \\
\hline $\mathfrak{p}_{3} F$ & $\lesseqgtr \sqrt{-2}\left[\left(2_{+}^{1+6} \cdot\left(\operatorname{Alt}_{8}: 2\right)\right]_{8}\right.$ & $\zeta_{10}\left[C_{10}\right]_{1} \underset{\sqrt{5}^{\prime}}{\otimes} \infty, 3\left[\mathrm{SL}_{2}(9)\right]_{2}$ \\
\hline $\mathfrak{p}_{2}^{2} \mathfrak{p}_{3} F$ & $\lesseqgtr \sqrt{-5}\left[i\left[\left(D_{8} \otimes C_{4}\right) \cdot S_{3}\right]_{2} \underset{i}{2_{+}}{ }_{\sqrt{5}, \infty}\left[\mathrm{SL}_{2}(5)\right]_{1}\right]_{8}$ & $\zeta_{10}\left[C_{10}\right]_{1} \underset{\sqrt{5}}{\otimes} \infty, 3\left[\mathrm{SL}_{2}(3) \stackrel{2}{\square} C_{3}\right]_{2}$ \\
\hline $\mathfrak{p}_{5} F$ & $\sqrt{-5}\left[C_{20}: C_{4}\right]_{4}^{2}$ & $\zeta_{10}\left[C_{10}\right]_{1}^{4}$ \\
\hline $\mathfrak{p}_{2}^{2} \mathfrak{p}_{5} F$ & $\sqrt{-5}\left[i\left[\left(D_{8} \otimes C_{4}\right) \cdot S_{3}\right]_{2} \stackrel{2}{\otimes} D_{10}\right]_{8}$ & $\zeta_{10}\left[C_{10}\right]_{1} \otimes F_{4}$ \\
\hline $\mathfrak{p}_{3} \mathfrak{p}_{5} F$ & $\lessgtr_{\sqrt{-2}}\left[\mathrm{GL}_{2}(3)\right]_{2} \otimes A_{4}$ & $\zeta_{10}\left[C_{10}\right]_{1}^{4}$ \\
\hline $\mathfrak{p}_{2}^{2} \mathfrak{p}_{3} \mathfrak{p}_{5} F$ & $\sqrt{-5}\left[i\left[\left(D_{8} \otimes C_{4}\right) \cdot S_{3}\right]_{2} \stackrel{2}{\otimes} D_{10}\right]_{8}$ & $\zeta_{10}\left[C_{60} \cdot\left(C_{2} \times C_{2}\right)\right]_{4}$ \\
\hline $\mathfrak{p}_{5}^{2} F$ & $\sqrt{-5}\left[\sqrt{5}, \infty\left[\mathrm{SL}_{2}(5)\right]_{1}{ }^{2_{+}} C_{4}\right]_{4}^{2}$ & $\left(\sqrt{5}, \infty\left[\mathrm{SL}_{2}(5)\right]_{1} \circ C_{5}\right)^{2}$ \\
\hline $\mathfrak{p}_{2}^{2} \mathfrak{p}_{5}^{2} F$ & $\sqrt{-5}\left[i\left[\left(D_{8} \otimes C_{4}\right) \cdot S_{3}\right]_{2}{\underset{i}{-}}_{\sqrt{5}, \infty}\left[\mathrm{SL}_{2}(5)\right]_{1}\right]_{8}$ & $\zeta_{10}\left[C_{10}\right]_{1} \underset{\sqrt{5}^{\prime}}{\otimes} \infty, 2\left[2_{-}^{1+4} \cdot \mathrm{Alt}_{5}\right]_{2}$ \\
\hline $\mathfrak{p}_{3} \mathfrak{p}_{5}^{2} F$ & $\lesseqgtr \sqrt{-2}\left[\left(2_{+}^{1+6} \cdot\left(\mathrm{Alt}_{8}: 2\right)\right]_{8}\right.$ & $\zeta_{10}\left[C_{10}\right]_{1} \underset{\sqrt{5}^{\prime}}{\otimes} \infty, 3\left[\mathrm{SL}_{2}(9)\right]_{2}$ \\
\hline $\mathfrak{p}_{2}^{2} \mathfrak{p}_{3} \mathfrak{p}_{5}^{2} F$ & $\lesseqgtr \sqrt{-5}\left[i\left[\left(D_{8} \otimes C_{4}\right) \cdot S_{3}\right]_{2} \stackrel{2}{\otimes} D_{10}\right]_{8}$ & $\zeta_{10}\left[C_{10}\right]_{1} \underset{\sqrt{5}^{\prime}}{\otimes} \infty, 3\left[\mathrm{SL}_{2}(3) \stackrel{2}{\square} C_{3}\right]_{2}$ \\
\hline
\end{tabular}




\begin{tabular}{|c|c|c|}
\hline form & $\mathbb{Q}(\sqrt{-10})$ & $\mathbb{Q}\left(\sqrt{2} \cdot\left(\zeta_{10}-\zeta_{10}^{-1}\right)\right)$ \\
\hline$F$ & $\sqrt{-10}\left[\sqrt{-2}\left[\mathrm{GL}_{2}(3)\right]_{2} \underset{\sqrt{2}}{2_{+}} \sqrt{5, \infty}\left[\mathrm{SL}_{2}(5)\right]_{1}\right]_{8}$ & $\sqrt{2} \cdot\left(\zeta_{10}-\zeta_{10}^{-1}\right)\left[\sqrt{2}, \infty\left[\tilde{S}_{4}\right]_{1}{\underset{\sqrt{5}}{\Phi^{\prime}}}_{\zeta_{10}}^{2-}\left[C_{10}\right]_{1}\right]_{4}$ \\
\hline $\mathfrak{p}_{2}^{2} F$ & $\lesseqgtr \sqrt{-10}\left[\sqrt{-2}\left[\mathrm{GL}_{2}(3)\right]_{2} \underset{\sqrt{2}}{2_{+}} \sqrt{5, \infty}\left[\mathrm{SL}_{2}(5)\right]_{1}\right]_{8}$ & $\sqrt{2} \cdot\left(\zeta_{10}-\zeta_{10}^{-1}\right)\left[\sqrt{2}, \infty\left[\tilde{S}_{4}\right]_{1} \underset{\sqrt{5}}{2_{+}} \zeta_{10}\left[C_{10}\right]_{1}\right]_{4}$ \\
\hline $\mathfrak{p}_{3} F$ & $\lesseqgtr_{i}\left[{ }_{\sqrt{-3}}\left[\operatorname{Sp}_{4}(3) \circ C_{3}\right]_{4} \stackrel{2(2)}{\otimes}{ }_{i}\left[C_{4}\right]_{1}\right]_{8}$ & $\succ_{\zeta_{10}}\left[C_{10}\right]_{1} \underset{\sqrt{5}^{\prime}}{\otimes} \infty, 3\left[\mathrm{SL}_{2}(9)\right]_{2}$ \\
\hline $\mathfrak{p}_{2}^{2} \mathfrak{p}_{3} F$ & $\lesseqgtr_{i}\left[\sqrt{-3}\left[\operatorname{Sp}_{4}(3) \circ C_{3}\right]_{4} \stackrel{2(2)}{\otimes}{ }_{i}\left[C_{4}\right]_{1}\right]_{8}$ & $\lesseqgtr \zeta_{10}\left[C_{10}\right]_{1} \otimes_{\sqrt{5}^{\prime}} \infty, 3\left[\mathrm{SL}_{2}(3) \stackrel{2}{\square} C_{3}\right]_{2}$ \\
\hline $\mathfrak{p}_{5} F$ & $\lesseqgtr \sqrt{-10}\left[\sqrt{-2}\left[\mathrm{GL}_{2}(3)\right]_{2} \stackrel{2}{\otimes} D_{10}\right]_{8}$ & 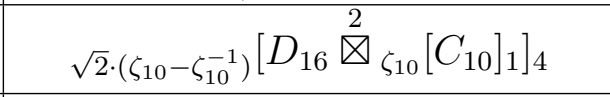 \\
\hline $\mathfrak{p}_{2}^{2} \mathfrak{p}_{5} F$ & $\sqrt{-10}\left[\sqrt{-2}\left[\mathrm{GL}_{2}(3)\right]_{2} \stackrel{2}{\otimes} D_{10}\right]_{8}$ & 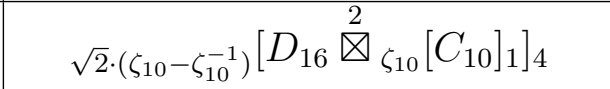 \\
\hline $\mathfrak{p}_{3} \mathfrak{p}_{5} F$ & $\Varangle_{i}\left[\left(2_{+}^{1+4} \otimes C_{4}\right) \cdot S_{6}\right]_{4}^{2}$ & $\succ_{\zeta_{10}}\left[C_{10}\right]_{1}^{4}$ \\
\hline $\mathfrak{p}_{2}^{2} \mathfrak{p}_{3} \mathfrak{p}_{5} F$ & $\lesseqgtr_{i}\left[\sqrt{-3}\left[\operatorname{Sp}_{4}(3) \circ C_{3}\right]_{4} \stackrel{2(2)}{\otimes}{ }_{i}\left[C_{4}\right]_{1}\right]_{8}$ & $\zeta_{\zeta_{10}}\left[C_{60} \cdot\left(C_{2} \times C_{2}\right)\right]_{4}$ \\
\hline $\mathfrak{p}_{5}^{2} F$ & $\sqrt{-10}\left[\sqrt{-2}\left[\mathrm{GL}_{2}(3)\right]_{2} \underset{\sqrt{2}}{\stackrel{2}{\otimes}} \sqrt{5}, \infty\left[\mathrm{SL}_{2}(5)\right]_{1}\right]_{8}$ & $\sqrt{2} \cdot\left(\zeta_{10}-\zeta_{10}^{-1}\right)\left[\sqrt{2}, \infty\left[\tilde{S}_{4}\right]_{1} \underset{\sqrt{5}}{\stackrel{2}{\otimes}} \zeta_{10}\left[C_{10}\right]_{1}\right]_{4}$ \\
\hline $\mathfrak{p}_{2}^{2} \mathfrak{p}_{5}^{2} F$ & $\lesseqgtr \sqrt{-10}\left[\sqrt{-2}\left[\mathrm{GL}_{2}(3)\right]_{2} \underset{\sqrt{2}}{\stackrel{2}{\otimes}} \sqrt{5}, \infty\left[\mathrm{SL}_{2}(5)\right]_{1}\right]_{8}$ & $\sqrt{2} \cdot\left(\zeta_{10}-\zeta_{10}^{-1}\right)\left[\sqrt{2}, \infty\left[\tilde{S}_{4}\right]_{1} \underset{\sqrt{5}}{\mathbb{\bigotimes}_{+}} \zeta_{10}\left[C_{10}\right]_{1}\right]_{4}$ \\
\hline $\mathfrak{p}_{3} \mathfrak{p}_{5}^{2} F$ & $\leq_{i}\left[\left(2_{+}^{1+4} \otimes C_{4}\right) \cdot S_{6}\right]_{4}^{2}$ & $\zeta_{\zeta_{10}}\left[C_{10}\right]_{1} \underset{\sqrt{5}}{\otimes} \infty, 3\left[\mathrm{SL}_{2}(9)\right]_{2}$ \\
\hline $\mathfrak{p}_{2}^{2} \mathfrak{p}_{3} \mathfrak{p}_{5}^{2} F$ & $\lesseqgtr_{i}\left[{ }_{\sqrt{-3}}\left[\mathrm{Sp}_{4}(3) \circ C_{3}\right]_{4} \stackrel{2(2)}{\otimes}{ }_{i}\left[C_{4}\right]_{1}\right]_{8}$ & $\lesseqgtr \zeta_{10}\left[C_{10}\right]_{1} \otimes_{\sqrt{5}^{\prime}} \infty, 3\left[\mathrm{SL}_{2}(3) \stackrel{2}{\square} C_{3}\right]_{2}$ \\
\hline
\end{tabular}

Theorem 4.9.7 Suppose $|U|=48$ and $\Pi(|G|)=\{2,3\}$ or there exists some $(L, F) \in$ $\mathcal{Z}(G) \times \mathcal{F}_{>0}(G)$ such that $F$ is integral on $L$ and $\Pi(\operatorname{det}(L, F)) \subseteq\{2,3\}$. Then $G$ is conjugate to one of

$$
\begin{aligned}
& { }_{i}\left[\left(2_{+}^{1+4} \otimes C_{4}\right) \cdot S_{6}\right]_{4}^{2},{ }_{i}\left[\left(D_{8} \otimes C_{4}\right) \cdot S_{3}\right]_{2}^{4}, \quad\left({ }_{i}\left[C_{4}\right]_{1} \otimes A_{2}\right)^{4},\left({ }_{i}\left[\left(D_{8} \otimes C_{4}\right) \cdot S_{3}\right]_{2} \otimes A_{2}\right)^{2}, \\
& \sqrt{-6}\left[\sqrt{2}, \infty\left[\tilde{S}_{4}\right]_{1}^{\left.\stackrel{2}{\square} C_{3}\right]_{4}^{2}, \sqrt{-6}[\sqrt{2}, \infty}\left[\tilde{S}_{4}\right]_{1}^{2} \square C_{3}\right]_{4}^{2}, \sqrt{-6}\left[D_{16} \stackrel{2}{\otimes} \underset{\sqrt{-3}}{2}\left[C_{6}\right]_{1}\right]_{4}^{2}, \\
& \sqrt{-3}\left[\mathrm{Sp}_{4}(3) \circ C_{3}\right]_{4}^{2},\left({ }_{\infty, 2}\left[\mathrm{SL}_{2}(3)\right]_{1} \circ C_{3}\right)^{4}, \sqrt{-3}\left[C_{6}\right]_{1}^{8},\left({ }_{\sqrt{-3}}\left[C_{6}\right]_{1} \otimes F_{4}\right)^{2}, \\
& \left.\sqrt{-2}\left[\infty, 2\left[2_{-}^{1+4} . \mathrm{Alt}_{5}\right]: 2\right]_{4}^{2}, \sqrt{-2}\left[F_{4}: 2\right]_{4}^{2}, \sqrt{3} \cdot\left(\zeta_{16}-\zeta_{16}^{-1}\right)\right)\left[\theta_{16}, \infty\left[Q_{32}\right]_{1} \stackrel{2}{\square} C_{3}\right]_{4},
\end{aligned}
$$

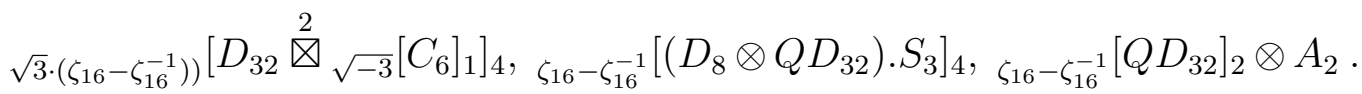

Proof: The commuting algebra $\operatorname{End}(\bar{U}) \simeq \mathbb{Q}\left(\zeta_{48}\right)$ has class number 1 . Thus $U$ fixes only one lattice $L$. There exists some $F \in \mathcal{F}_{>0}(U)$ that is integral on $L$ with $\operatorname{det}(L, F)=1$. So by Table 2.5.3. $G$ must be conjugate to $\operatorname{Aut}_{K}\left(L, \mathfrak{p}_{2}^{2 a} \mathfrak{p}_{3}^{b} F\right)$ for some $0 \leq a \leq 2$, $b \in\{0,1\}$ and $K \in\left\{\mathbb{Q}(i), \mathbb{Q}(\sqrt{-2}), \mathbb{Q}(\sqrt{-3}), \mathbb{Q}(\sqrt{-6}), \mathbb{Q}\left(\sqrt{3}\left(\zeta_{16}-\zeta_{16}^{-1}\right)\right), \mathbb{Q}\left(\zeta_{16}-\zeta_{16}^{-1}\right)\right\}$. 


\begin{tabular}{|c|c|c|c|}
\hline form & $\mathbb{Q}(i)$ & $\mathbb{Q}(\sqrt{-6})$ & $\mathbb{Q}(\sqrt{-3})$ \\
\hline$F$ & ${ }_{i}\left[\left(2_{+}^{1+4} \otimes C_{4}\right) \cdot S_{6}\right]_{4}^{2}$ & $\sqrt{-6}\left[\sqrt{2}, \infty\left[\tilde{S}_{4}\right]_{1}^{2} \square C_{3}\right]_{4}^{2}$ & $\sqrt{-3}\left[\operatorname{Sp}_{4}(3) \circ C_{3}\right]_{4}^{2}$ \\
\hline $\mathfrak{p}_{2}^{2} F$ & ${ }_{i}\left[\left(D_{8} \otimes C_{4}\right) \cdot S_{3}\right]_{2}^{4}$ & $\lesseqgtr \sqrt{-6}\left[\sqrt{2}, \infty\left[\tilde{S}_{4}\right]_{1}{ }^{2} \square C_{3}\right]_{4}^{2}$ & $\lesseqgtr\left(\infty, 2\left[\mathrm{SL}_{2}(3)\right]_{1} \circ C_{3}\right)^{4}$ \\
\hline $\mathfrak{p}_{2}^{4} F$ & ${ }_{i}\left[\left(D_{8} \otimes C_{4}\right) \cdot S_{3}\right]_{2}^{4}$ & $\sqrt{-6}\left[\sqrt{2}, \infty\left[\tilde{S}_{4}\right]_{1}{ }^{2+} C_{3}\right]_{4}^{2}$ & $\left(\infty, 2\left[\mathrm{SL}_{2}(3)\right]_{1} \circ C_{3}\right)^{4}$ \\
\hline $\mathfrak{p}_{3} F$ & $\left({ }_{i}\left[C_{4}\right]_{1} \otimes A_{2}\right)^{4}$ & $\sqrt{-6}\left[D_{16} \stackrel{2}{\otimes} \underset{\sqrt{-3}}{ }\left[C_{6}\right]_{1}\right]_{4}^{2}$ & $\sqrt{-3}\left[C_{6}\right]_{1}^{8}$ \\
\hline $\mathfrak{p}_{2}^{2} \mathfrak{p}_{3} F$ & $\lesseqgtr\left({ }_{i}\left[C_{4}\right]_{1} \otimes A_{2}\right)^{4}$ & $\lesseqgtr_{\sqrt{-6}}\left[D_{16} \stackrel{2}{\otimes}_{\sqrt{-3}}\left[C_{6}\right]_{1}\right]_{4}^{2}$ & $\lesseqgtr \sqrt{-3}\left[C_{6}\right]_{1}^{8}$ \\
\hline $\mathfrak{p}_{2}^{4} \mathfrak{p}_{3} F$ & $\left({ }_{i}\left[\left(D_{8} \otimes C_{4}\right) \cdot S_{3}\right]_{2} \otimes A_{2}\right)^{2}$ & $\sqrt{-6}\left[D_{16} \stackrel{2}{\otimes} \underset{\sqrt{-3}}{ }\left[C_{6}\right]_{1}\right]_{4}^{2}$ & $\lesseqgtr\left(\sqrt{-3}\left[C_{6}\right]_{1} \otimes F_{4}\right)^{2}$ \\
\hline
\end{tabular}

The remaining groups are

$$
\operatorname{Aut}_{\mathbb{Q}(\sqrt{-2})}\left(L, \mathfrak{p}_{2}^{2 a} \mathfrak{p}_{3}^{b} F\right) \leq \begin{cases}\sqrt{-2}\left[{ }_{\infty}\left[2_{-}^{1+4} \cdot \mathrm{Alt}_{5}\right]: 2\right]_{4}^{2} & \text { if } a=0 \\ \sqrt{-2}\left[F_{4}: 2\right]_{4}^{2} & \text { if } a \neq 0\end{cases}
$$

with equality if and only if $b=0$ and $a \neq 1$

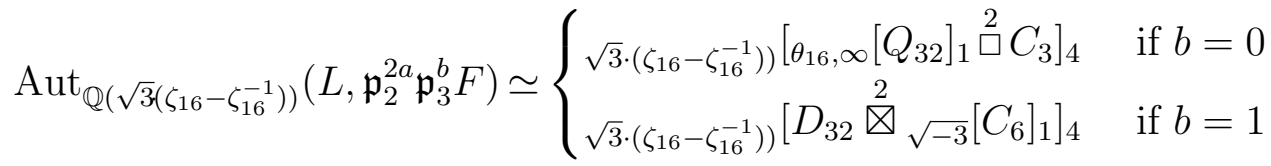

$$
\begin{aligned}
& \operatorname{Aut}_{\mathbb{Q}\left(\zeta_{16}-\zeta_{16}^{-1}\right)}\left(L, \mathfrak{p}_{2}^{2 a} \mathfrak{p}_{3}^{b} F\right) \simeq\left\{\begin{array}{ll}
\zeta_{16}-\zeta_{16}^{-1}\left[\left(D_{8} \otimes Q D_{32}\right) . S_{3}\right]_{4} & \text { if } b=0 \\
\zeta_{16}-\zeta_{16}^{-1}\left[Q D_{32}\right]_{2} \otimes A_{2} & \text { if } b=1
\end{array} .\right.
\end{aligned}
$$

So the result follows.

Theorem 4.9.8 Suppose $|U|=60$ and $\Pi(|G|)=\{2,3,5\}$ or there exists some $(L, F) \in \mathcal{Z}(G) \times \mathcal{F}_{>0}(G)$ such that $F$ is integral on $L$ and $\Pi(\operatorname{det}(L, F)) \subseteq\{2,3,5\}$. Then $G$ is conjugate to one of

$$
\begin{aligned}
& { }_{i}\left[C_{4}\right]_{1} \otimes E_{8},{ }_{i}\left[\left(2_{+}^{1+6} \otimes C_{4}\right) \cdot \operatorname{Sp}_{6}(2)\right]_{8},{ }_{i}\left[\left(2_{+}^{1+4} \otimes C_{4}\right) \cdot S_{6}\right]_{4} \otimes A_{2}, \\
& i\left[\sqrt{-3}\left[\operatorname{Sp}_{4}(3) \circ C_{3}\right]_{4} \stackrel{2(2)}{\otimes}{ }_{i}\left[C_{4}\right]_{1}\right]_{8}, \sqrt{-3}\left[\operatorname{Sp}_{4}(3) \circ C_{3}\right]_{4}^{2}, \infty, 2\left[2_{-}^{1+6} . O_{6}^{-}(2)\right]_{4} \circ C_{3} \text {, } \\
& \sqrt{-3}\left[C_{6}\right]_{1} \otimes E_{8}, \sqrt{-3}\left[\operatorname{Sp}_{4}(3) \circ C_{3}\right]_{4} \underset{\sqrt{-3}}{\otimes} \infty\left[\mathrm{SL}_{2}(3)\right]_{1}, \infty, 5\left[\mathrm{SL}_{2}(5) \underset{\sqrt{5}}{\stackrel{2}{\otimes}} D_{10}\right]_{4} \circ C_{3}, \\
& \left({ }_{\infty, 2}\left[\mathrm{SL}_{2}(3)\right]_{1} \circ C_{3}\right) \otimes A_{4},\left({ }_{\sqrt{-3}}\left[C_{6}\right]_{1} \otimes A_{4}\right)^{2}, \sqrt{-3}\left[C_{60} \cdot\left(C_{4} \times C_{2}\right)\right]_{8}, \\
& \left(\infty, 5\left[\mathrm{SL}_{2}(5): 2\right]_{2} \circ C_{3}\right)^{2}, \infty, 2\left[\mathrm{SL}_{2}(5) \stackrel{2(2)}{\otimes} D_{8}\right]_{4} \circ C_{3}, \sqrt{-3}\left[C_{6}\right]_{1} \otimes\left[\left(\mathrm{SL}_{2}(5) \stackrel{2}{\square} \mathrm{SL}_{2}(5)\right): 2\right]_{8} \text {, } \\
& \left(\infty, 2\left[\mathrm{SL}_{2}(3)\right]_{1} \circ C_{3}\right) \underset{\sqrt{-3}}{\otimes} \infty, 5\left[\mathrm{SL}_{2}(5): 2\right]_{2}, \sqrt{-5}\left[\left(\left(\mathrm{SL}_{2}(5) \circ \mathrm{SL}_{2}(5)\right): 2\right) \stackrel{2}{凶}_{i}\left[C_{4}\right]_{1}\right]_{8}, \\
& \left.\sqrt{-5}\left[\infty, 5\left[\mathrm{SL}_{2}(5) \underset{\sqrt{5}}{\stackrel{2}{\otimes}} D_{10}\right]_{4}: 2\right]_{8}, \sqrt{-5}\left[\left(\mathrm{SL}_{2}(5) \circ \mathrm{SL}_{2}(5)\right): 2\right) \stackrel{2_{+}}{\otimes}{ }_{i}\left[C_{4}\right]_{1}\right]_{8},
\end{aligned}
$$




$$
\begin{aligned}
& \sqrt{-5}\left[i\left[\left(D_{8} \otimes C_{4}\right) \cdot S_{3}\right]_{2} \underset{i}{2_{+}} \underset{\sqrt{5}, \infty}{ }\left[\mathrm{SL}_{2}(5)\right]_{1}\right]_{8}, \sqrt{-5}\left[i\left[\left(D_{8} \otimes C_{4}\right) . S_{3}\right]_{2} \stackrel{2}{\otimes} D_{10}\right]_{8}, \\
& \sqrt{-5}\left[i\left[\left(D_{8} \otimes C_{4}\right) \cdot S_{3}\right]_{2} \underset{i}{2_{-}} \underset{\sqrt{5}, \infty}{ }\left[\mathrm{SL}_{2}(5)\right]_{1}\right]_{8}, \sqrt{-15}\left[\sqrt{5}, \infty\left[\mathrm{SL}_{2}(5)\right]_{1} \stackrel{2_{-}}{\square} C_{3}\right]_{4}^{2}, \\
& \sqrt{-15}\left[\left(\sqrt{5}, \infty\left[\mathrm{SL}_{2}(5)\right]_{1} \circ C_{3}\right) \underset{\sqrt{5}}{\stackrel{2}{\otimes}} D_{10}\right]_{8}, \sqrt{-15}\left[\sqrt{5}, \infty\left[\mathrm{SL}_{2}(5)\right]_{1} \stackrel{2_{+}}{\square} C_{3}\right]_{4}^{2}, \\
& \sqrt{-15}\left[\left(\left(\mathrm{SL}_{2}(5) \circ \mathrm{SL}_{2}(5)\right): 2\right) \stackrel{2}{\unrhd}_{\sqrt{-3}}\left[C_{6}\right]_{1}\right]_{8}, \sqrt{-15}\left[C_{30}: C_{4}\right]_{4}^{2}, \\
& \sqrt{-15}\left[\left(\left(\mathrm{SL}_{2}(5) \circ \mathrm{SL}_{2}(5)\right): 2\right) \stackrel{2_{+}}{\otimes} \underset{\sqrt{-3}}{ }\left[C_{6}\right]_{1}\right]_{8},\left(\sqrt{5}, \infty\left[\mathrm{SL}_{2}(5)\right]_{1} \circ C_{5}\right)^{2} \text {, } \\
& \zeta_{10}\left[C_{10}\right]_{1} \underset{\sqrt{5}^{\prime}}{\otimes} \otimes_{2}\left[2_{-}^{1+4} \cdot \mathrm{Alt}_{5}\right]_{2}, \quad\left(\sqrt{5}, \infty\left[\mathrm{SL}_{2}(5)\right]_{1} \circ C_{5}\right) \otimes A_{2}, \zeta_{10}\left[C_{10}\right]_{1} \underset{\sqrt{5}^{\prime}}{\otimes} \underset{\infty, 3}{ }\left[\mathrm{SL}_{2}(3) \stackrel{2}{\square} C_{3}\right]_{2}, \\
& \zeta_{10}\left[C_{10}\right]_{1} \underset{\sqrt{5}}{\otimes}\left(\left(\mathrm{SL}_{2}(5) \circ \mathrm{SL}_{2}(5)\right): 2\right), \zeta_{10}\left[C_{10}\right]_{1} \otimes F_{4}, \zeta_{10}\left[C_{30}: 2\right]_{2}^{2}, \zeta_{10}\left[C_{60} \cdot\left(C_{2} \times C_{2}\right)\right]_{4}
\end{aligned}
$$

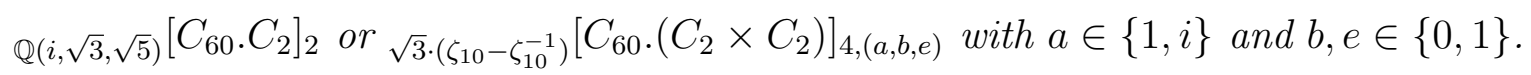

Proof: Let $C:=\operatorname{End}(\bar{U}) \simeq \mathbb{Q}\left(\zeta_{60}\right)$ and $K \simeq \mathbb{Q}\left(\theta_{60}\right)$ be its maximal totally real subfield. Both have class number 1 . Hence $U$ fixes up to isomorphism only one lattice $L$. There exists some $F \in \mathcal{F}_{>0}(U)$ that is integral on $L$ such that $(L, F) \simeq E_{8}^{2}$. Clearly $U$ is not maximal finite. Hence, the maximal totally real subfield of $\operatorname{End}(\bar{G})$ is properly contained in $K$. Thus by the proof of Corollary 2.2.12, we may assume that $G$ fixes a form $F^{\prime} \in \mathcal{F}_{>0}(U)$ that is integral on $L$ with $\Pi\left(\operatorname{det}\left(L, F^{\prime}\right)\right) \subseteq\{2,3,5\} \cup \bigcup_{k<K} \Pi(k)=$ $\{2,3,5,11\}$. (Note that this is a huge improvement over $\tilde{\Pi}(K, 60)=\{2,3,5,11,59\}$ ). Denote by $\mathfrak{p}_{11}^{(i)}(1 \leq i \leq 4)$ the four prime ideals over 11. By Table 2.5.3. $G$ is conjugate to $\operatorname{Aut}_{K^{\prime}}\left(L, \mathfrak{p}_{2}^{a} \mathfrak{p}_{3}^{b} \mathfrak{p}_{5}^{c} \prod_{i=1}^{4}\left(\mathfrak{p}_{11}^{(i)}\right)^{d_{i}} F\right)$ for some $a, b \in\{0,1\}, 0 \leq c \leq 2, d_{i} \geq 0$ and $K^{\prime} \in\left\{\mathbb{Q}(i), \mathbb{Q}(\sqrt{-3}), \mathbb{Q}(\sqrt{-5}), \mathbb{Q}(\sqrt{-15}), \mathbb{Q}\left(\zeta_{10}\right), \mathbb{Q}\left(\sqrt{3} \cdot\left(\zeta_{10}-\zeta_{10}^{-1}\right)\right)\right\}$.

There exists some $\sigma_{i} \in \operatorname{Gal}(K / \mathbb{Q})$ such that $\sigma_{i}\left(\mathfrak{p}_{11}^{(1)}\right)=\mathfrak{p}_{11}^{(i)}$ and one finds that $\sigma_{i}$ is conjugation by some $x_{i} \in N_{\mathrm{GL}_{16}(\mathbb{Q})}(N) \cap \mathrm{GL}(L)$. Since $\sigma_{i}$ necessarily fixes $\mathfrak{p}_{2}, \mathfrak{p}_{3}$ and $\mathfrak{p}_{5}$, we may assume that $d_{1} \geq d_{i}$ for all $i$. Furthermore, restricting to normalized lattices (see Definition 2.2.4 yields the inequalities $d_{i} \leq 1$ and $\sum_{i} d_{i} \leq 2$.

First, we handle the cases $d_{1}=0$ : Let $a, b \in\{0,1\}$ and $0 \leq c \leq 2$, then

$$
\operatorname{Aut}_{\mathbb{Q}(i)}\left(L, \mathfrak{p}_{2}^{a} \mathfrak{p}_{3}^{b} \mathfrak{p}_{5}^{c} F\right) \leq \begin{cases}i\left[C_{4}\right]_{1} \otimes E_{8} & \text { if } a=b=0 \\ i\left[\left(2_{+}^{1+6} \otimes C_{4}\right) \cdot \operatorname{Sp}_{6}(2)\right]_{8} & \text { if }(a, b)=(1,0) \\ i\left[\left(2_{+}^{1+4} \otimes C_{4}\right) \cdot S_{6}\right]_{4} \otimes A_{2} & \text { if }(a, b)=(0,1) \\ { }_{i}\left[{ }_{\sqrt{-3}}\left[\operatorname{Sp}_{4}(3) \circ C_{3}\right]_{4} \stackrel{2(2)}{\otimes}{ }_{i}\left[C_{4}\right]_{1}\right]_{8} & \text { if } a=b=1\end{cases}
$$

and equality holds if and only if $c=0$. 
The groups involving $\mathbb{Q}(\sqrt{-3})$ are

\begin{tabular}{|c|c|}
\hline form & $\mathbb{Q}(\sqrt{-3})$ \\
\hline$F$ & $\sqrt{-3}\left[\operatorname{Sp}_{4}(3) \circ C_{3}\right]_{4}^{2}$ \\
\hline $\mathfrak{p}_{2} F$ & $\infty, 2\left[2_{-}^{1+6} \cdot O_{6}^{-}(2)\right]_{4} \circ C_{3}$ \\
\hline $\mathfrak{p}_{3} F$ & $\sqrt{-3}\left[C_{6}\right]_{1} \otimes E_{8}$ \\
\hline $\mathfrak{p}_{2} \mathfrak{p}_{3} F$ & $\sqrt{-3}\left[\mathrm{Sp}_{4}(3) \circ C_{3}\right]_{4} \underset{\sqrt{-3}}{\otimes} \infty, 2\left[\mathrm{SL}_{2}(3)\right]_{1}$ \\
\hline $\mathfrak{p}_{5} F$ & $\infty, 5\left[\mathrm{SL}_{2}(5) \underset{\sqrt{5}}{\stackrel{2}{\otimes}} D_{10}\right]_{4} \circ C_{3}$ \\
\hline $\mathfrak{p}_{2} \mathfrak{p}_{5} F$ & $\left(\infty, 2\left[\mathrm{SL}_{2}(3)\right]_{1} \circ C_{3}\right) \otimes A_{4}$ \\
\hline $\mathfrak{p}_{3} \mathfrak{p}_{5} F$ & $\left(\sqrt{-3}\left[C_{6}\right]_{1} \otimes A_{4}\right)^{2}$ \\
\hline $\mathfrak{p}_{2} \mathfrak{p}_{3} \mathfrak{p}_{5} F$ & $\sqrt{-3}\left[C_{60} \cdot\left(C_{4} \times C_{2}\right)\right]_{8}$ \\
\hline $\mathfrak{p}_{5}^{2} F$ & $\left(\infty, 5\left[\mathrm{SL}_{2}(5): 2\right]_{2} \circ C_{3}\right)^{2}$ \\
\hline $\mathfrak{p}_{2} \mathfrak{p}_{5}^{2} F$ & $\infty, 2\left[\mathrm{SL}_{2}(5) \stackrel{2(2)}{\otimes} D_{8}\right]_{4} \circ C_{3}$ \\
\hline $\mathfrak{p}_{3} \mathfrak{p}_{5}^{2} F$ & $\sqrt{-3}\left[C_{6}\right]_{1} \otimes\left[\left(\mathrm{SL}_{2}(5) \stackrel{2}{\square} \mathrm{SL}_{2}(5)\right): 2\right]_{8}$ \\
\hline $\mathfrak{p}_{2} \mathfrak{p}_{3} \mathfrak{p}_{5}^{2} F$ & $\left(\infty, 2\left[\mathrm{SL}_{2}(3)\right]_{1} \circ C_{3}\right) \underset{\sqrt{-3}}{\otimes} \infty, 5\left[\mathrm{SL}_{2}(5): 2\right]_{2}$ \\
\hline
\end{tabular}

which are all s.i.m.f..

Similarly

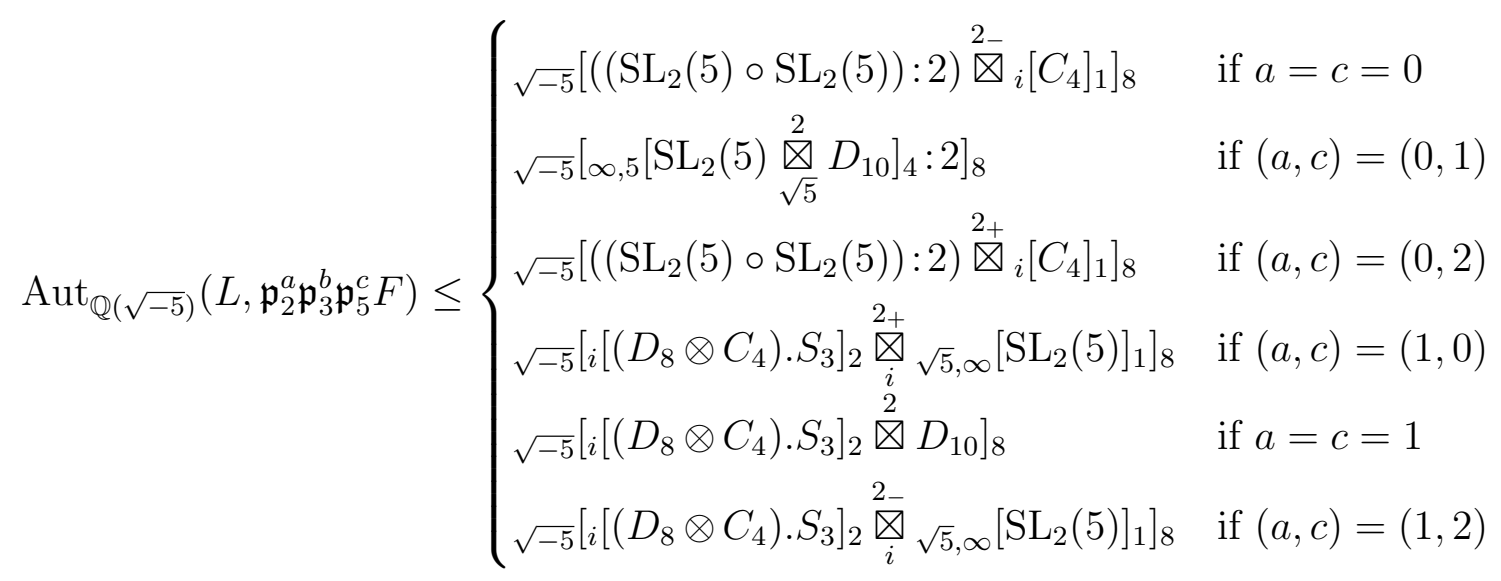

and equality holds if and only if $b=0$.

The groups involving $\mathbb{Q}(\sqrt{-15})$ are $\operatorname{Aut}_{\mathbb{Q}(\sqrt{-15})}\left(L, \mathfrak{p}_{2}^{a} \mathfrak{p}_{3}^{b} \mathfrak{p}_{5}^{c} F\right) \leq$

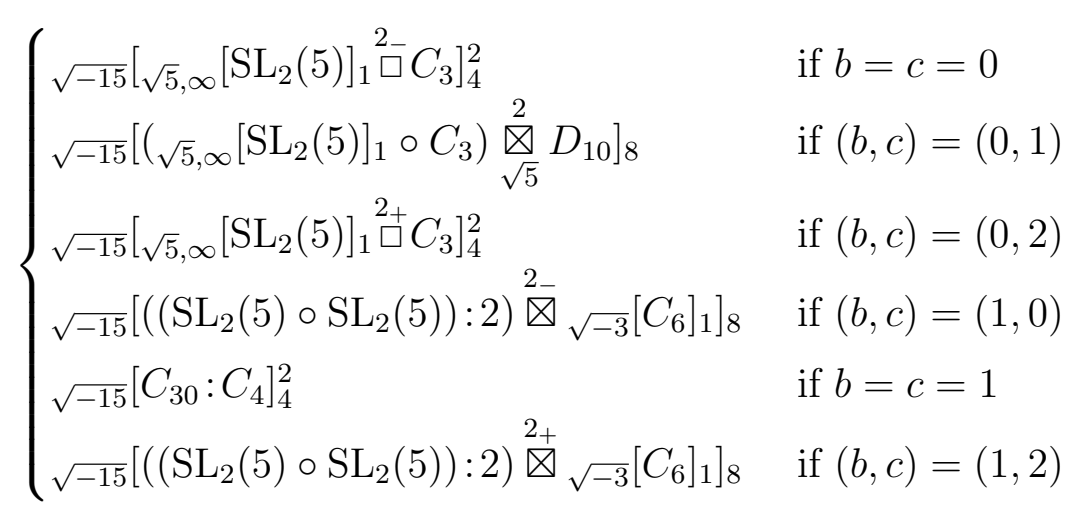

and equality holds if and only if $a=0$. The remaining two fields yield 


\begin{tabular}{|c|c|c|}
\hline form & $\mathbb{Q}\left(\zeta_{10}\right)$ & $\mathbb{Q}\left(\sqrt{3} \cdot\left(\zeta_{10}-\zeta_{10}^{-1}\right)\right)$ \\
\hline $\bar{F}$ & $\left(\overline{\sqrt{5}}_{5}\left[\mathrm{SL}_{2}(5)\right]_{1} \circ C_{5}\right)^{2}$ & $\overline{\sqrt{3} \cdot\left(\zeta_{10}-\zeta_{10}^{-1}\right)}\left[C_{60} \cdot\left(C_{2} \times C_{2}\right)\right]_{4,(1,-1,1)}$ \\
\hline $\mathfrak{p}_{2} F$ & $\zeta_{10}\left[C_{10}\right]_{1} \underset{\sqrt{5}^{\prime}}{\otimes} \infty, 2\left[2_{-}^{1+4} \cdot \mathrm{Alt}_{5}\right]_{2}$ & $\sqrt{3} \cdot\left(\zeta_{10}-\zeta_{10}^{-1}\right)\left[C_{60} \cdot\left(C_{2} \times C_{2}\right)\right]_{4,(i,-1,1)}$ \\
\hline $\mathfrak{p}_{3} F$ & $\left(\sqrt{5}, \infty\left[\mathrm{SL}_{2}(5)\right]_{1} \circ C_{5}\right) \otimes A_{2}$ & $\sqrt{3} \cdot\left(\zeta_{10}-\zeta_{10}^{-1}\right)\left[C_{60} \cdot\left(C_{2} \times C_{2}\right)\right]_{4,(1,1,-1)}$ \\
\hline $\mathfrak{p}_{2} \mathfrak{p}_{3} F$ & $\zeta_{10}\left[C_{10}\right]_{1} \underset{\sqrt{5}^{\prime}}{\otimes^{\prime}, 3}\left[\mathrm{SL}_{2}(3) \stackrel{2}{\square} C_{3}\right]_{2}$ & $\sqrt{3} \cdot\left(\zeta_{10}-\zeta_{10}^{-1}\right)\left[C_{60} \cdot\left(C_{2} \times C_{2}\right)\right]_{4,(i, 1,-1)}$ \\
\hline $\mathfrak{p}_{5} F$ & $\zeta_{10}\left[C_{10}\right]_{1} \underset{\sqrt{5}}{\otimes}\left(\left(\mathrm{SL}_{2}(5) \circ \mathrm{SL}_{2}(5)\right): 2\right)$ & 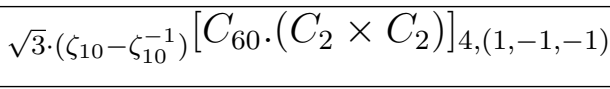 \\
\hline $\mathfrak{p}_{2} \mathfrak{p}_{5} F$ & $\zeta_{10}\left[C_{10}\right]_{1} \otimes F_{4}$ & $\sqrt{3} \cdot\left(\zeta_{10}-\zeta_{10}^{-1}\right)\left[C_{60} \cdot\left(C_{2} \times C_{2}\right)\right]_{4,(i,-1,-1)}$ \\
\hline $\mathfrak{p}_{3} \mathfrak{p}_{5} F$ & $\zeta_{10}\left[C_{30}: 2\right]_{2}^{2}$ & $\sqrt{3} \cdot\left(\zeta_{10}-\zeta_{10}^{-1}\right)\left[C_{60} \cdot\left(C_{2} \times C_{2}\right)\right]_{4,(1,1,1)}$ \\
\hline $\mathfrak{p}_{2} \mathfrak{p}_{3} \mathfrak{p}_{5} F$ & ${ }_{\zeta_{10}}\left[C_{60} \cdot\left(C_{2} \times C_{2}\right)\right]_{4}$ & $\sqrt{3} \cdot\left(\zeta_{10}-\zeta_{10}^{-1}\right)\left[C_{60} \cdot\left(C_{2} \times C_{2}\right)\right]_{4,(i, 1,1)}$ \\
\hline
\end{tabular}

The fields $\mathbb{Q}\left(\zeta_{10}\right)$ and $\mathbb{Q}\left(\sqrt{3} \cdot\left(\zeta_{10}-\zeta_{10}^{-1}\right)\right)$ have only one proper subfield, which is isomorphic to $\mathbb{Q}(\sqrt{5})$. Thus, these groups are easily be checked to be s.i.m.f. by computing the automorphism groups of the $G$-invariant lattices wrt. the full form space. Moreover, we don't have to check the forms $\mathfrak{p}_{2}^{a} \mathfrak{p}_{3}^{b} \mathfrak{p}_{5}^{2} F$ since $\mathfrak{p}_{5}^{2} F=\frac{5+\sqrt{5}}{2} F$.

Suppose now $d_{1}=1$ and $d_{2}=d_{3}=d_{4}=0$ :

For each $d \in\{1,3,5,15\}$ the group $\operatorname{Aut}_{\mathbb{Q}(\sqrt{-d})}\left(L_{i}, \mathfrak{p}_{2}^{a} \mathfrak{p}_{3}^{b} \mathfrak{p}_{5}^{c} \mathfrak{p}_{11}^{(1)} F\right)$ is conjugate to $\mathbb{Q}(i, \sqrt{3}, \sqrt{5})\left[C_{60} \cdot C_{2}\right]_{2}$ which we have already proven to be s.i.m.f. in Lemma 4.9.2.

For $\alpha \in\left\{\zeta_{10}, \sqrt{3} \cdot\left(\zeta_{10}-\zeta_{10}^{-1}\right)\right\}$ the groups $\operatorname{Aut}_{\mathbb{Q}(\alpha)}\left(L, \mathfrak{p}_{2}^{a} \mathfrak{p}_{3}^{b} \mathfrak{p}_{5}^{c} \mathfrak{p}_{11}^{(1)} F\right)$ are conjugate to an extension of $U$ by $C_{2}$ with commuting algebra $\mathbb{Q}\left(\zeta_{10}, \sqrt{3}\right)$. The extension is split if and only if $c$ is odd. The nonsplit extension is properly contained in $\left(\sqrt{5}, \infty\left[\mathrm{SL}_{2}(5)\right]_{1} \circ C_{5}\right)^{2}$ and the split extension is a subgroup of ${ }_{\zeta_{10}}\left[C_{10}\right]_{1} \underset{\sqrt{5}}{\otimes}\left(\left(\mathrm{SL}_{2}(5) \circ \mathrm{SL}_{2}(5)\right): 2\right)$.

Finally suppose $d_{1}=1$ and $\sum_{i=1}^{4} d_{i}=2$. Then, for every minimal totally complex subfield $K^{\prime}$ of $K$, $\operatorname{Aut}_{K^{\prime}}\left(L, \mathfrak{p}_{2}^{a} \mathfrak{p}_{3}^{b} \mathfrak{p}_{5}^{c} \prod_{i=1}^{4}\left(\mathfrak{p}_{11}^{(i)}\right)^{d_{i}} F\right)$ is conjugate to a proper subgroup of $\operatorname{Aut}_{K^{\prime}}\left(L, \mathfrak{p}_{2}^{a} \mathfrak{p}_{3}^{b} \mathfrak{p}_{5}^{c} F\right)$.

\subsubsection{The case $O_{17}(G)=1$ and $O_{5}(G) \neq 1$}

Lemma 4.9.9 If $E(G)$ is conjugate to ${ }_{\sqrt{5}, \infty}\left[\mathrm{SL}_{2}(5)\right]_{1}$, then $G$ is conjugate to one of

$$
\begin{gathered}
\sqrt{-5}\left[\infty, 5\left[\mathrm{SL}_{2}(5) \underset{\sqrt{5}}{\stackrel{2}{\otimes}} D_{10}\right]_{4}: 2\right]_{8}, \infty, 5\left[\mathrm{SL}_{2}(5) \underset{\sqrt{5}}{\stackrel{2}{\otimes}} D_{10}\right]_{4} \circ C_{3}, \\
\sqrt{-15}\left[\left(\sqrt{5}, \infty\left[\mathrm{SL}_{2}(5)\right]_{1} \circ C_{3}\right) \underset{\sqrt{5}}{\otimes} D_{10}\right]_{8}, \quad\left(\sqrt{5}, \infty\left[\mathrm{SL}_{2}(5)\right]_{1} \circ C_{5}\right) \otimes A_{2} .
\end{gathered}
$$

Proof: The group $G$ contains a normal subgroup $N$ conjugate to $\mathrm{SL}_{2}(5) \circ C_{5}$. Suppose $N$ is self-centralizing. Then $\operatorname{Out}(N) \simeq\langle\alpha, \beta\rangle \simeq C_{2} \times C_{4}$. Both, $\alpha$ and $\beta$ cannot be realized in $\mathrm{GL}_{16}(\mathbb{Q})$ alone. Hence by Lemma 2.2.1. $G=\langle N, \alpha \beta\rangle$ with $(\alpha \beta)^{4}= \pm 1$. 
One of these groups is realizeable in dimension 8. The other gives an irreducible group $G$ with commuting algebra $\mathcal{Q}_{\infty, 5}$. But the torsion subgroup of the (up to isomorphism unique) maximal order of $\mathcal{Q}_{\infty, 5}$ is $C_{6}$. Thus $N$ is not self-centralizing.

So we may assume that $G$ contains an irreducible subgroup $H:=N \otimes C_{m}$ with $m \in\{3,4\}$. In both cases $G$ must therefore contain an irreducible cyclic subgroup of order 60. The commuting algebra of $H$ is isomorphic to $C:=\mathbb{Q}\left(\zeta_{10}, \zeta_{m}\right)$. The torsion subgroup of $\mathbb{Z}_{C}^{*}$ is $C_{30}$ if $m=3$ and $C_{20}$ otherwise. Hence $G / C_{G}(N) N \leq \operatorname{Out}(N) \simeq C_{2} \times C_{4}$ implies $\Pi(|G|)=\{2,3,5\}$. So the result follows from Theorem 4.9.8.

Lemma 4.9.10 If $O_{3}(G) \neq 1$ then $G$ is conjugate to one of

$$
\begin{aligned}
& \left(\sqrt{5}, \infty\left[\mathrm{SL}_{2}(5)\right]_{1} \circ C_{5}\right) \otimes A_{2}, \infty, 5\left[\mathrm{SL}_{2}(5) \underset{\sqrt{5}}{\stackrel{2}{\otimes}} D_{10}\right]_{4} \circ C_{3}, \\
& \sqrt{-15}\left[\left(\sqrt{5}, \infty\left[\mathrm{SL}_{2}(5)\right]_{1} \circ C_{3}\right) \underset{\sqrt{5}}{\stackrel{2}{\otimes}} D_{10}\right]_{8}, \zeta_{10}\left[C_{60} \cdot\left(C_{2} \times C_{2}\right)\right]_{4}, \quad \sqrt{-3}\left[C_{60} \cdot\left(C_{4} \times C_{2}\right)\right]_{8}, \\
& \zeta_{10}\left[C_{10}\right]_{1} \underset{\sqrt{5}^{\prime}}{\otimes} \infty, 3\left[\mathrm{SL}_{2}(3) \stackrel{2}{\square} C_{3}\right]_{2}, \mathbb{Q}(i, \sqrt{3}, \sqrt{5})\left[C_{60} \cdot C_{2}\right]_{2}
\end{aligned}
$$

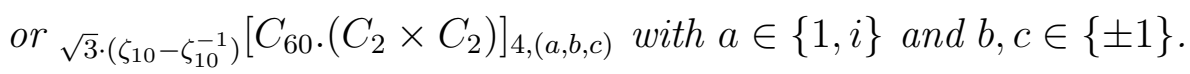

Proof: Table 2.5.2 shows that $O_{3}(G) \simeq C_{3}$ and $O_{2}(G)$ embeds into $\mathrm{GL}_{2}\left(\mathbb{Q}\left(\zeta_{15}\right)\right)$. Thus $O_{2}(G) \simeq C_{2}, C_{4}, D_{8}$ or $Q_{8}$. If $O_{2}(G) \neq C_{2}$ then $G$ contains an irreducible cyclic subgroup of order 60 and $\Pi(|G|)=\{2,3,5\}$. Theorem 4.9 .8 gives precisely the claimed groups.

If $F(G) \simeq \pm C_{15}$ then $C:=C_{G}(F(G))$ embeds into $\mathrm{GL}_{2}\left(\mathbb{Q}\left(\zeta_{15}\right)\right)$. Table 2.5.1 shows that $E(G)$ is either trivial or conjugate to $\sqrt{5}, \infty\left[\mathrm{SL}_{2}(5)\right]_{1}$. The latter case has been handled in the lemma before. So we may now suppose that $F^{*}(G)= \pm C_{15}$. Let $C:=C_{G}\left(O_{3}(G)\right)$. Then $[G: C] \leq 2$ and $C / F(G) \leq \operatorname{Out}\left(C_{5}\right)$. Hence $C$ is conjugate to one of $\pm C_{15}, D_{10} \otimes C_{6}, Q_{20} \circ C_{3},\left(C_{5}: C_{4}\right) \otimes C_{6},\left(C_{10} \cdot C_{4}\right) \circ C_{3}$. In any case, $C$ is rationally reducible and the commuting algebra of a direct summand contains a third root of unity. This contradicts Lemma 2.2.1.

Lemma 4.9.11 If $O_{3}(G)=1$ and $O_{2}(G)=Q_{8}$ then $G$ is conjugate to one of

$$
\begin{aligned}
& \sqrt{-10}\left[\sqrt{-2}\left[\mathrm{GL}_{2}(3)\right]_{2} \stackrel{2}{\otimes} D_{10}\right]_{8}, \sqrt{2} \cdot\left(\zeta_{10}-\zeta_{10}^{-1}\right)\left[\sqrt{2}, \infty\left[\tilde{S}_{4}\right]_{1} \underset{\sqrt{5}^{\prime}}{\stackrel{2}{\otimes}} \zeta_{10}\left[C_{10}\right]_{1}\right]_{4}, \\
& \sqrt{2} \cdot\left(\zeta_{10}-\zeta_{10}^{-1}\right)\left[\sqrt{2}, \infty\left[\tilde{S}_{4}\right]_{1} \underset{\sqrt{5}^{\prime}}{\stackrel{2_{+}}{\otimes}} \zeta_{10}\left[C_{10}\right]_{1}\right]_{4} \cdot
\end{aligned}
$$

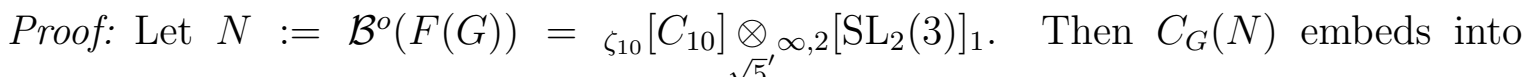
$\mathrm{GL}_{2}\left(\mathbb{Q}\left(\zeta_{10}\right)\right)$. It follows from Table 2.5.1 that $E(G)=1$. So $G / N \leq \operatorname{Out}(N) \simeq C_{4} \times C_{2}$ and $[G: N] \geq 4$ by Lemma 2.2.1. In particular, $\Pi(|G|)=\{2,3,5\}$. Let $U:=$ $C_{G}\left(O_{5}(G)\right) \unlhd G$. There are three possibilities: 
- If $U \simeq{ }_{\zeta_{10}}\left[C_{10}\right]_{1} \otimes \sqrt{-2}\left[\mathrm{GL}_{2}(3)\right]_{2}$, then $U$ fixes up to isomorphism one lattice $L$. Further $C:=\operatorname{End}(\bar{U}) \simeq \mathbb{Q}\left(\zeta_{10}, \sqrt{-2}\right)$ has $K:=\mathbb{Q}\left(\sqrt{-2} \cdot\left(\zeta_{10}-\zeta_{10}^{-1}\right)\right)$ as maximal real subfield. There exists some $F \in \mathcal{F}_{>0}(U)$ that is integral on $L$ with $\operatorname{det}(L, F)=1$. Since $\operatorname{End}_{\mathbb{Z} U}(L)$ is the maximal order of $C$ and since $\mathrm{Nr}_{C / K}\left(\mathbb{Z}_{C}^{*}\right)=\mathbb{Z}_{K,>0}^{*}$, we may restrict ourselves to one class of totally positive units. It follows from Table 2.5.4 that $G$ is conjugate to one of $\operatorname{Aut}_{K^{\prime}}\left(L, \mathfrak{p}_{2}^{a} \mathfrak{p}_{5}^{b} F\right)$ for some $(a, b) \in\{(0,0),(1,1),(0,2)\}$ and $K^{\prime} \in\left\{\mathbb{Q}(\sqrt{-2}), \mathbb{Q}(\sqrt{-10}), \mathbb{Q}\left(\zeta_{10}\right)\right\}$. These are

\begin{tabular}{|c|c|c|}
\hline form & $\mathbb{Q}(\sqrt{-2})$ & $\mathbb{Q}\left(\zeta_{10}\right)$ \\
\hline$F$ & $\overline{\sqrt{-2}}\left[2_{+}^{1+6} \cdot\left(\operatorname{Alt}_{8}: 2\right)\right]_{8}$ & $\left.\overline{\left({ }^{5}, \infty\right.}{ }\left[\mathrm{SL}_{2}(5)\right]_{1} \circ C_{5}\right)^{2}$ \\
\hline $\mathfrak{p}_{2} \mathfrak{p}_{5} F$ & $\sqrt{-2}\left[\mathrm{GL}_{2}(3)\right]_{2} \otimes A_{4}$ & $\zeta_{10}\left[C_{10}\right]_{1} \otimes F_{4}$ \\
\hline $\mathfrak{p}_{5}^{2} F$ & $\sqrt{-2}\left[\mathrm{GL}_{2}(3)\right]_{2} \underset{\sqrt{-2}}{\otimes} \infty, 5\left[\mathrm{SL}_{2}(5): 2\right]_{2}$ & $\left(\sqrt{5, \infty}\left[\mathrm{SL}_{2}(5)\right]_{1} \circ C_{5}\right)^{2}$ \\
\hline form & $\mathbb{Q}(\sqrt{-10})$ & \\
\hline$F$ & $\overline{\sqrt{-10}}\left[\sqrt{-2}\left[\mathrm{GL}_{2}(3)\right]_{2} \stackrel{2_{+}}{\underset{\sqrt{-2}}{\mathrm{~N}} \sqrt{5}, \infty}\left[\mathrm{SL}_{2}(5)\right]_{1}\right]_{8}$ & \\
\hline $\mathfrak{p}_{2} \mathfrak{p}_{5} F$ & $\sqrt{-10}\left[\sqrt{-2}\left[\mathrm{GL}_{2}(3)\right]_{2} \stackrel{2}{\otimes} D_{10}\right]_{8}$ & \\
\hline $\mathfrak{p}_{5}^{2} F$ & $\sqrt{-10}\left[\sqrt{-2}\left[\mathrm{GL}_{2}(3)\right]_{2} \underset{\sqrt{-2}}{\stackrel{2}{\otimes}} \sqrt{5}, \infty\left[\mathrm{SL}_{2}(5)\right]_{1}\right]_{8}$ & \\
\hline
\end{tabular}

All groups are s.i.m.f. but only $\sqrt{-10}\left[\sqrt{-2}\left[\mathrm{GL}_{2}(3)\right]_{2} \stackrel{2}{\otimes} D_{10}\right]_{8}$ has the correct Fitting subgroup.

- If $U \simeq \sqrt{2}, \infty\left[\tilde{S}_{4}\right]_{1} \underset{\sqrt{5}}{\otimes^{\prime}} \zeta_{10}\left[C_{10}\right]_{1}$, then again $U$ fixes only one lattice $L$. The commuting algebra $C:=\operatorname{End}(\bar{U}) \simeq \mathbb{Q}\left(\zeta_{10}, \sqrt{2}\right)$ has $K:=\mathbb{Q}(\sqrt{2}, \sqrt{5})$ as maximal real subfield. Again there exists some $F \in \mathcal{F}_{>0}(U)$ such that $F$ is integral on $L$ with $\operatorname{det}(L, F)=1$. Further, there exists some $\sigma(\operatorname{Gal}(K / \mathbb{Q}))$ that interchanges the two prime ideals over $3 \mathbb{Z}_{K}$ and $\sigma$ is conjugation by some $x \in N_{\mathrm{GL}_{16}}(\mathbb{Q})(U) \cap \mathrm{GL}(L)$. So by Table 2.5.4. $G$ is conjugate to $\operatorname{Aut}_{K^{\prime}}\left(L, \mathfrak{p}_{2}^{a} \mathfrak{p}_{3}^{b} F\right)$ for some $a, b \in\{0,1\}$ and $K^{\prime} \in\left\{\mathbb{Q}\left(\zeta_{10}\right), \mathbb{Q}\left(\sqrt{2} \cdot\left(\zeta_{10}-\zeta_{10}^{-1}\right)\right)\right\}$. Note that since $K^{\prime}$ has $\mathbb{Q}(\sqrt{5})$ as maximal totally real subfield, we do not have to check $\mathfrak{p}_{5}$ since $\mathfrak{p}_{5} F=\frac{5+\sqrt{5}}{2} F$. These groups are:

\begin{tabular}{|c|c|c|}
\hline form & $\mathbb{Q}\left(\zeta_{10}\right)$ & $\mathbb{Q}\left(\sqrt{2} \cdot\left(\zeta_{10}-\zeta_{10}^{-1}\right)\right)$ \\
\hline$F$ & $\left(\sqrt{5}, \infty\left[\mathrm{SL}_{2}(5)\right]_{1} \circ C_{5}\right)^{2}$ & $\sqrt{2} \cdot\left(\zeta_{10}-\zeta_{10}^{-1}\right)\left[\sqrt{2}, \infty\left[\tilde{S}_{4}\right]_{1}{\underset{\sqrt{5}}{\prime^{\prime}} \zeta_{10}}_{\zeta_{10}}\left[C_{10}\right]_{1}\right]_{4}$ \\
\hline $\mathfrak{p}_{2} F$ & $\zeta_{10}\left[C_{10}\right]_{1} \underset{\sqrt{5}^{\prime}}{\otimes} \infty, 2\left[2_{-}^{1+4} \cdot \mathrm{Alt}_{5}\right]_{2}$ & $\sqrt{2} \cdot\left(\zeta_{10}-\zeta_{10}^{-1}\right)\left[\sqrt{2}, \infty\left[\tilde{S}_{4}\right]_{1} \underset{\sqrt{5}^{\prime}}{2_{+}} \zeta_{10}\left[C_{10}\right]_{1}\right]_{4}$ \\
\hline $\mathfrak{p}_{3} F$ & 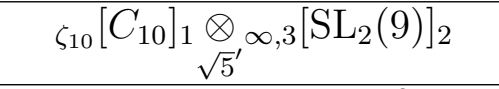 & $\bar{U}$ \\
\hline$\left(L, \mathfrak{p}_{2} \mathfrak{p}_{3} F\right)$ & $\zeta_{10}\left[C_{10}\right]_{1} \underset{\sqrt{5}^{\prime}}{\otimes}, \infty, 3\left[\mathrm{SL}_{2}(3) \stackrel{2}{\square} C_{3}\right]_{2}$ & $U$ \\
\hline
\end{tabular}

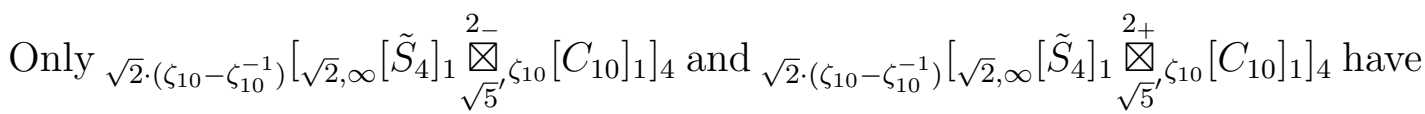
the required Fitting subgroup. 
- If $U=N$, then $G / N \simeq C_{4}$. More precisely $G$ is one of the four groups $G=\langle N, \alpha\rangle$ where $\alpha$ induces an outer automorphism of order 4 on $O_{5}(G)$ and either

○ $\alpha^{4}=I_{16}$ and $\alpha \in C_{G}\left(\mathcal{B}^{\circ}\left(O_{2}(G)\right)\right)$. But then $G$ can be extended by torsion elements in $\operatorname{End}(\bar{G}) \simeq \mathcal{Q}_{\infty, 2}$.

$\circ \alpha^{4}=-I_{16}$ and $\alpha \in C_{G}\left(\mathcal{B}^{\circ}\left(O_{2}(G)\right)\right)$. This group is for example properly contained in $\left(\infty, 2\left[\mathrm{SL}_{2}(3)\right]_{1} \circ C_{3}\right) \underset{\sqrt{-3}}{\otimes} \infty, 5\left[\mathrm{SL}_{2}(5): 2\right]_{2}$.

$\circ \alpha^{4}=I_{16}$ and $\alpha$ induces the outer automorphism of $\mathcal{B}^{\circ}\left(O_{2}(G)\right)$. This group embeds into $\mathrm{GL}_{8}(\mathbb{Q})$.

$\circ \alpha^{4}=-I_{16}$ and $\alpha$ induces the outer automorphism of $\mathcal{B}^{\circ}\left(O_{2}(G)\right)$. This group is properly contained in $\infty, 5\left[\mathrm{SL}_{2}(5) \underset{\sqrt{2}}{\stackrel{2}{\otimes}} D_{10}\right]_{4}$.

Thus the result follows.

Lemma 4.9.12 If $O_{3}(G)=1$ then $O_{2}(G)$ is not isomorphic to $C_{4}$ or $D_{8}$.

Proof: In both cases $G$ would contain a normal subgroup $N \simeq C_{20}$. The centralizer $C:=C_{G}(N)$ embeds into $\mathrm{GL}_{2}\left(\mathbb{Q}\left(\zeta_{20}\right)\right)$. Hence $E(G)$ is either conjugate to $\sqrt{5}, \infty\left[\mathrm{SL}_{2}(5)\right]_{1}$ or trivial. The first case contradicts Lemma 4.9.9.

Thus $F(G)$ is self-centralizing. Hence $G / F(G) \leq \operatorname{Out}(F(G)) \simeq C_{2} \times C_{4}$. It follows from Lemma 2.2.1 that $[G: F(G)]>2$. If $g \in C_{G}\left(O_{5}(G)\right) \backslash F(G)$ then $g \in O_{2}(G)$. In particular, this shows $G / F(G) \simeq C_{4}$. This leaves the two cases:

- $F(G) \simeq C_{20}$. By group cohomology, MAGMA finds that $G$ must be isomorphic to $\left\langle a, b, \alpha \mid a^{5}, b^{4},[a, b], a^{\alpha}=a^{2}, b^{\alpha}=b^{\epsilon}, \alpha^{4}=b^{k}\right\rangle$ for some $(\epsilon, k) \in$ $\{(1,0),(-1,0),(-1,2),(1,2),(1,1)\}$. The first three groups embed into $G_{8}(\mathbb{Q})$ with $\mathbb{Q}(i)$ and $\mathbb{Q}(\sqrt{-5})$ as endomorphism rings. The fourth is properly contained in $\sqrt{-10}\left[\sqrt{-2}\left[\mathrm{GL}_{2}(3)\right]_{2} \stackrel{2}{\otimes} D_{10}\right]_{8}$ and the last one only has a rational irreducible representation in dimension 32 .

- $F(G) \simeq C_{5} \times D_{8}$. In this case $G$ must be isomorphic to

$$
\left\langle a, b, c, \alpha \mid a^{5}, b^{4},[a, b],[a, c], b^{c}=b^{-1}, c^{2}, a^{\alpha}=a^{2}, b^{\alpha}=b^{-1}, c^{\alpha}=b^{r} c, \alpha^{4}=b^{2 s}\right\rangle
$$

for some $r, s \in\{0,1\}$. Thus $U:=\langle a, b, \alpha\rangle$ is the group discussed above (with $\epsilon=-1$ and $k \in\{0,2\})$. In particular $U$ is irreducible and has a imaginary quadratic field as commuting algebra. This contradicts Lemma 2.2.1.

So the result follows. 


\section{Lemma 4.9.13}

(a) $\mathrm{O}_{2}(G)$ is neither isomorphic to $C_{8}, Q D_{16}$ nor $Q_{16}$.

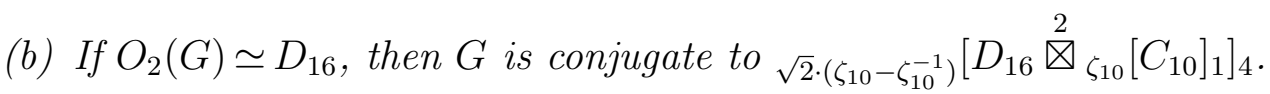

Proof: In any case, $G$ contains an irreducible cyclic subgroup $U \leq F(G)$ of order 40 . Thus $F(G)$ is self-centralizing and hence $\Pi(|G|)=\{2,3,5\}$. So the result follows from Theorem 4.9.6.

\section{Lemma 4.9.14}

(a) If $\mathrm{O}_{2}(G) \simeq 2_{+}^{1+4}$, then $G$ is conjugate to $\zeta_{10}\left[C_{10}\right]_{1} \otimes F_{4}$.

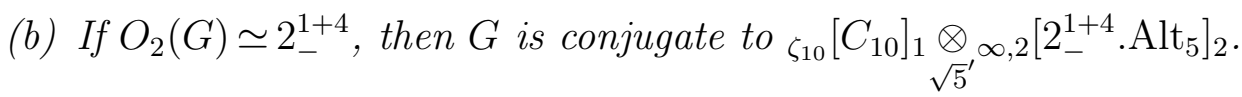

Proof: We have to prove $\mathcal{B}^{\circ}(F(G))=G$ in both cases. The group $\mathcal{B}^{\circ}(F(G))$ is irreducible, fixes 2 lattices and has $\mathbb{Q}\left(\zeta_{10}\right)$ as endomorphism ring. Hence the claim is verified by computing the automorphism groups of these lattices wrt. the full form space.

Lemma 4.9.15 If $O_{2}(G) \simeq D_{8} \otimes C_{4}$ then $G \simeq \sqrt{-5}\left[i\left[\left(D_{8} \otimes C_{4}\right) . S_{3}\right]_{2} \stackrel{2}{\otimes} D_{10}\right]_{8}$.

Proof: The group $G$ contains an irreducible normal subgroup $N:=\mathcal{B}^{\circ}(F(G))=$ ${ }_{i}\left[\left(D_{8} \otimes C_{4}\right) \cdot S_{3}\right]_{2} \otimes \zeta_{10}\left[C_{10}\right]_{1}$. The commuting algebra of $N$ is isomorphic to $\mathbb{Q}\left(\zeta_{20}\right)$ and $N$ fixes only one lattice $L$. Thus $N$ is self-centralizing and $\Pi(|G|)=\{2,3,5\}$. Further we find some $F \in \mathcal{F}_{>0}(G)$ that is integral on $L$ with $\operatorname{det}(L, F)=2^{8}$. Restricting to normalized lattices (see Definition 2.2.4) it follows from Table 2.5.3 that $G$ is conjugate to $\operatorname{Aut}_{K}\left(L, \mathfrak{p}_{5}^{a} F\right)$ for some $0 \leq a \leq 2$ and $K \in\left\{\mathbb{Q}(i), \mathbb{Q}(\sqrt{-5}), \mathbb{Q}\left(\zeta_{10}\right)\right\}$. These groups are

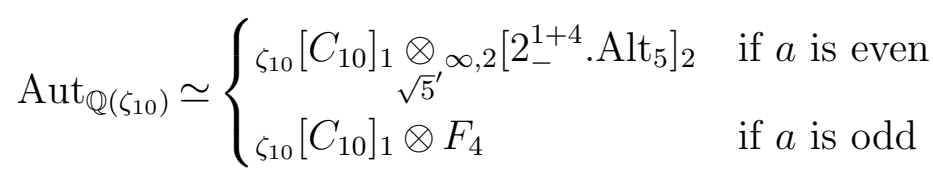

and

\begin{tabular}{|c|c|c|}
\hline & $\mathbb{Q}(i)$ & $\mathbb{Q}(\sqrt{-5})$ \\
\hline \hline$F$ & ${ }_{i}\left[\left(2_{+}^{1+6} \otimes C_{4}\right) \cdot \operatorname{Sp}_{2}(6)\right]_{8}$ & $\sqrt{-5}\left[i\left[\left(D_{8} \otimes C_{4}\right) \cdot S_{3}\right]_{2} \underset{i}{2_{+}} \mathrm{SL}_{2}(5)\right]_{8}$ \\
\hline$p_{5} F$ & $\varlimsup_{i}\left[\left(2_{+}^{1+6} \otimes C_{4}\right) \cdot \mathrm{Sp}_{2}(6)\right]_{8}$ & $\sqrt{-5}\left[i\left[\left(D_{8} \otimes C_{4}\right) \cdot S_{3}\right]_{2} \stackrel{2}{\otimes} D_{10}\right]_{8}$ \\
\hline$p_{5}^{2} F$ & ${ }_{i}\left[\left(2_{+}^{1+6} \otimes C_{4}\right) \cdot \mathrm{Sp}_{2}(6)\right]_{8}$ & $\sqrt{-5}\left[i\left[\left(D_{8} \otimes C_{4}\right) \cdot S_{3}\right]_{2} \underset{i}{\otimes} \mathrm{SL}_{2}(5)\right]_{8}$ \\
\hline
\end{tabular}

Only $\sqrt{-5}\left[i\left[\left(D_{8} \otimes C_{4}\right) \cdot S_{3}\right]_{2} \stackrel{2}{\otimes} D_{10}\right]_{8}$ has the correct Fitting subgroup. 
Lemma 4.9.16 If $F(G) \simeq C_{10}$, then $G$ is conjugate to $\zeta_{10}\left[C_{10}\right]_{1} \underset{\sqrt{5}}{\otimes}\left(\left(\mathrm{SL}_{2}(5) \circ \mathrm{SL}_{2}(5)\right): 2\right)$ or $\zeta_{10}\left[C_{10}\right]_{1} \underset{\sqrt{5}}{\otimes} \infty, 3\left[\mathrm{SL}_{2}(9)\right]_{2}$.

Proof: Since $C_{G}(F(G))$ embeds into $\mathbb{Q}\left(\zeta_{10}\right)^{2 \times 2}$, it follows from Table 2.5.1 that $E(G)$ is conjugate to $\mathrm{Alt}_{5}, \mathrm{SL}_{2}(5) \circ \mathrm{SL}_{2}(5), \infty, 3\left[\mathrm{SL}_{2}(9)\right]_{2}$ or ${ }_{5}, \infty\left[\mathrm{SL}_{2}(5)\right]_{1}$. The latter case contradicts Lemma 4.9.9.

- If $E(G) \simeq \simeq_{\infty, 3}\left[\mathrm{SL}_{2}(9)\right]_{2}$ then $F^{*}(G) \simeq \zeta_{10}\left[C_{10}\right]_{1} \underset{\sqrt{5}^{\prime}}{\otimes} \infty_{3,3}\left[\mathrm{SL}_{2}(9)\right]_{2}$ is s.i.m.f..

- If $E(G) \simeq \mathrm{SL}_{2}(5) \circ \mathrm{SL}_{2}(5)$ then $\mathcal{B}^{o}\left(F^{*}(G)\right) \simeq \zeta_{10}\left[C_{10}\right]_{1} \underset{\sqrt{5}}{\otimes}\left(\left(\mathrm{SL}_{2}(5) \circ \mathrm{SL}_{2}(5)\right): 2\right)$ is already s.i.m.f..

- If $E(G) \simeq \mathrm{Alt}_{5}$ then $\mathcal{B}^{\circ}\left(F^{*}(G)\right) \simeq \zeta_{10}\left[C_{10}\right]_{1} \otimes A_{4}$ has $\mathbb{Q}\left(\zeta_{10}\right)$ as commuting algebra and fixes up to isomorphism 5 lattices. By computing their automorphism groups (wrt. the full form space), one finds that $G$ is only contained in $\zeta_{10}\left[C_{10}\right]_{1} \underset{\sqrt{5}}{\otimes}\left(\left(\mathrm{SL}_{2}(5) \circ \mathrm{SL}_{2}(5)\right): 2\right)$.

This proves the claim.

\subsubsection{The case $O_{17}(G)=O_{5}(G)=1$ and $O_{3}(G) \neq 1$}

Let $G<\operatorname{Sp}_{16}(\mathbb{Q})$ be s.p.i.m.f. such that $O_{3}(G) \neq 1$ and $O_{p}(G)=1$ for all primes $p>3$. Then $O_{3}(G) \simeq C_{3}$ and $O_{2}(G)$ is isomorphic to $C_{2}, C_{4}, D_{8}, Q_{8}, C_{8}, D_{16}, Q_{16}, Q D_{16}$, $D_{8} \otimes C_{4}, 2_{+}^{1+4}, 2_{-}^{1+4}, C_{16}, D_{32}, Q_{32}, Q D_{32}, D_{8} \otimes C_{8}, D_{8} \otimes D_{16}, D_{8} \otimes Q D_{16}, D_{8} \otimes Q_{16}$, $2_{+}^{1+4} \otimes C_{4}, 2_{+}^{1+6}$ or $2_{-}^{1+6}$ by Table 2.5.2.

Again, we discuss all possible cases.

Lemma 4.9.17 If $O_{2}(G) \simeq 2_{-}^{1+6}$ then $G$ is conjugate to $\infty_{, 2}\left[2_{-}^{1+6} \cdot O_{6}^{-}(2)\right]_{4} \circ C_{3}$.

Proof: Follows from Lemma 4.9.4.

Lemma 4.9.18 $\mathrm{O}_{2}(G)$ is not conjugate to $2_{+}^{1+6}$.

Proof: Otherwise $G$ would contain $\mathcal{B}^{\circ}(F(G)) \simeq 2_{+}^{1+6}$. Alt $\otimes_{\sqrt{-3}}\left[C_{6}\right]_{1}$ as a normal subgroup. This group fixes two lattices and has $\mathbb{Q}(\sqrt{-3})$ as commuting algebra. One easily checks that it is only contained in the s.i.m.f. group $\sqrt{-3}\left[C_{6}\right]_{1} \otimes E_{8}$. 
Lemma 4.9.19 If $O_{2}(G) \simeq 2_{+}^{1+4} \otimes C_{4}$, then $G$ is conjugate to ${ }_{i}\left[\left(2_{+}^{1+4} \otimes C_{4}\right) \cdot S_{6}\right]_{4} \otimes A_{2}$.

Proof: Otherwise $G$ would contain the irreducible normal subgroup $N:=\mathcal{B}^{\circ}(F(G))$ which is conjugate to ${ }_{i}\left[\left(2_{+}^{1+4} \otimes C_{4}\right) \cdot S_{6}\right]_{4} \otimes{ }_{\sqrt{-3}}\left[C_{6}\right]_{1}$. Since $G / N \leq C_{2} \times C_{2}$ we may assume $\Pi(|G|)=\{2,3,5\}$. Let $C:=\operatorname{End}(\bar{U}) \simeq \mathbb{Q}\left(\zeta_{12}\right)$ and $K \simeq \mathbb{Q}(\sqrt{3})$ be its totally real subfield. The group $N$ fixes up to isomorphism only one lattice $L$ and there exists some $F \in \mathcal{F}_{>0}(N)$ that is integral on $L$ with $\operatorname{det}(L, F)=2^{8}$. Since $\mathbb{Z}_{K,>0}^{*}=\operatorname{Nr}_{C / K}\left(\mathbb{Z}_{C}^{*}\right)$ we can restrict ourselves to one class of totally positive units. By Table 2.5.4. $G$ must be conjugate to $\operatorname{Aut}_{\mathbb{Q}(\sqrt{-d})}\left(L, \mathfrak{p}_{2}^{-a} \mathfrak{p}_{3}^{a} F\right)$ for some $a \in\{0,1\}$ and $d \in\{1,3\}$.

\begin{tabular}{|c|c|c|}
\hline form & $\mathbb{Q}(i)$ & $\mathbb{Q}(\sqrt{-3})$ \\
\hline \hline$F$ & ${ }_{i}\left[\left(2_{+}^{1+6} \otimes C_{4}\right) \cdot \operatorname{Sp}_{6}(2)\right]_{8}$ & $\infty, 2\left[2_{-}^{1+6} \cdot O_{6}^{-}(2)\right]_{4} \circ C_{3}$ \\
\hline $\mathfrak{p}_{2}^{-1} \mathfrak{p}_{3} F$ & ${ }_{i}\left[\left(2_{+}^{1+4} \otimes C_{4}\right) \cdot S_{6}\right]_{4} \otimes A_{2}$ & $\sqrt{-3}\left[C_{6}\right]_{1} \otimes E_{8}$ \\
\hline
\end{tabular}

The result follows by checking the Fitting subgroups.

\section{Lemma 4.9.20}

$O_{2}(G)$ is not conjugate to $D_{8} \otimes C_{8}, D_{8} \otimes Q D_{16}, D_{8} \otimes D_{16}$ or $D_{8} \otimes Q_{16}$.

Proof: In any case $G$ contains a normal irreducible subgroup $H$ conjugate to $C_{3} \otimes D_{8} \otimes C_{8}$. Thus also $N:=\mathcal{B}^{\circ}(H) \unlhd G$ and $N$ is conjugate to $C_{3} \otimes\left(D_{8} \otimes C_{8}\right) . S_{3}$. The commuting algebra $C$ of $N$ is isomorphic to $\mathbb{Q}\left(\zeta_{24}\right)$ with $K \simeq \mathbb{Q}\left(\theta_{24}\right)$ as maximal totally real subfield. Further $N$ fixes up to isomorphism only one lattice $L$ and one finds some $F \in \mathcal{F}_{>0}(N)$ that is integral on $L$ with $\operatorname{det}(L, F)=1$. From $G / F(G) \leq \operatorname{Out}(F(G))$ it follows that $\Pi(|G|)=\{2,3\}$. Since $\operatorname{End}_{\mathbb{Z} N}(N)$ is the maximal order in $C$ and since $\mathbb{Z}_{K,>0}^{*}=\mathrm{Nr}_{C / K}\left(\mathbb{Z}_{C}^{*}\right)$, we may restrict ourselves to one class of totally positive units. If follows from Table 2.5.3, that $G$ is conjugate to $\operatorname{Aut}_{\mathbb{Q}(\sqrt{-d})}\left(L, \mathfrak{p}_{2}^{2 a} \mathfrak{p}_{3}^{b} F\right)$ for some $a, b \in\{0,1\}$ and $d \in\{1,2,3,6\}$. These are

\begin{tabular}{|c|c|c|}
\hline form & $\mathbb{Q}(i)$ & $\mathbb{Q}(\sqrt{-2})$ \\
\hline$F$ & ${ }_{i}\left[\left(2_{+}^{1+4} \otimes C_{4}\right) \cdot S_{6}\right]_{4}^{2}$ & $\sqrt{-2}\left[2_{+}^{1+6} \cdot\left(\mathrm{Alt}_{8}: 2\right)\right]_{8}$ \\
\hline $\mathfrak{p}_{2}^{2} F$ & ${ }_{i}\left[\left(2_{+}^{1+6} \otimes C_{4}\right) \cdot \operatorname{Sp}_{6}(2)\right]_{8}$ & $\sqrt{-2}\left[\infty, 2\left[2_{-}^{1+6} \cdot O_{6}^{-}(2)\right]_{4}: 2\right]_{8}$ \\
\hline $\mathfrak{p}_{3} F$ & $i\left[\left(2_{+}^{1+4} \otimes C_{4}\right) \cdot S_{6}\right]_{4} \otimes A_{2}$ & $\overbrace{\sqrt{-2}}\left[2_{+}^{1+6} \cdot\left(\mathrm{Alt}_{8}: 2\right)\right]_{8}$ \\
\hline $\mathfrak{p}_{2}^{2} \mathfrak{p}_{3} F$ & $\left({ }_{i}\left[\left(D_{8} \otimes C_{4}\right) \cdot S_{3}\right]_{2} \otimes A_{2}\right)^{2}$ & $\lesseqgtr \sqrt{-2}\left[\infty, 2\left[2_{-}^{1+6} \cdot O_{6}^{-}(2)\right]_{4}: 2\right]_{8}$ \\
\hline & $\mathbb{Q}(\sqrt{-3})$ & $\mathbb{Q}(\sqrt{-6})$ \\
\hline$F$ & $\sqrt{-3}\left[\operatorname{Sp}_{4}(3) \circ C_{3}\right]_{4}^{2}$ & $\sqrt{-6}\left[\infty, 2\left[2_{-}^{1+4} \cdot \mathrm{Alt}_{5}\right]_{2} \underset{i}{\bigotimes_{i}^{(3)}} A_{2}\right]_{8}$ \\
\hline $\mathfrak{p}_{2}^{2} F$ & $\infty, 2\left[2_{-}^{1+6} \cdot O_{6}^{-}(2)\right]_{4} \circ C_{3}$ & $\sqrt{-6}\left[\infty, 2\left[2_{-}^{1+4} \cdot \mathrm{Alt}_{5}\right]_{2} \underset{i}{\underset{\mathrm{X}}{2(3)}} \infty, 3\left[\tilde{S}_{3}\right]_{1}\right]_{8}$ \\
\hline $\mathfrak{p}_{3} F$ & $\sqrt{-3}\left[C_{6}\right]_{1} \otimes E_{8}$ & $\sqrt{-6}\left[F_{4} \stackrel{2}{\otimes}_{\infty, 3}\left[\tilde{S}_{3}\right]_{1}\right]_{8}$ \\
\hline $\mathfrak{p}_{2}^{2} \mathfrak{p}_{3} F$ & $\left(\sqrt{-3}\left[C_{6}\right]_{1} \otimes F_{4}\right)^{2}$ & $\sqrt{-6}\left[\left(F_{4} \otimes A_{2}\right): 2\right]_{8}$ \\
\hline
\end{tabular}

No group has the correct Fitting subgroup. 


\section{Lemma 4.9.21}

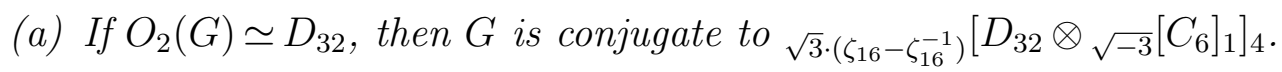

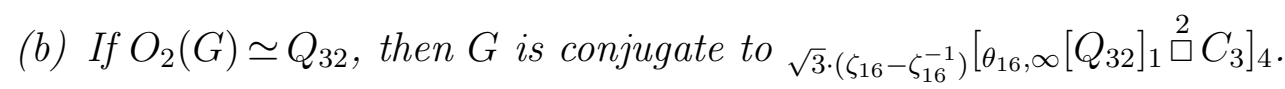

(c) If $O_{2}(G) \simeq Q D_{32}$, then $G$ is conjugate to ${ }_{\zeta_{16}-\zeta_{16}^{-1}}\left[Q D_{32}\right]_{2} \otimes A_{2}$.

(d) $\mathrm{O}_{2}(G)$ is not conjugate to $C_{16}$.

Proof: In all cases, $F(G)$ contains an irreducible self-centralizing cyclic subgroup of order 48. Furher, $\operatorname{Out}(F(G))$ is a 2-group which shows $\Pi(|G|)=\{2,3\}$. So the result follows from Theorem 4.9.7.

Lemma 4.9.22 If $O_{2}(G) \simeq 2_{-}^{1+4}$, then $G$ is conjugate to $\left.\sqrt{-6}_{\infty, 2}\left[2_{-}^{1+4} \cdot \mathrm{Alt}_{5}\right]_{2} \stackrel{2(3)}{\otimes} A_{2}\right]_{8}$ or $\sqrt{-6}\left[\infty, 2\left[2_{-}^{1+4} \cdot \mathrm{Alt}_{5}\right]_{2} \underset{i}{\stackrel{2(3)}{\bigotimes}} \infty\left[\tilde{S}_{3}\right]_{1}\right]_{8}$.

Proof: $G$ contains the normal subgroup $N:=\mathcal{B}^{\circ}\left(O_{2}(G)\right) \circ C_{3} \simeq{ }_{\infty, 2}\left[2_{-}^{1+4} \text {. Alt }\right]_{2} \circ C_{3}$. By Lemma 2.2.1 we get $G / N \simeq C_{2} \times C_{2}$. Thus $C_{G}\left(O_{3}(G)\right)$ is conjugate to $N_{1}:=$ $\sqrt{-2}\left[\infty, 2\left[2_{-}^{1+4} \cdot \mathrm{Alt}_{5}\right]_{2}: 2\right]_{4} \otimes C_{3}$ or $N_{2}:=\left({ }_{\infty, 2}\left[2_{-}^{1+4} \cdot \mathrm{Alt}_{5}\right]_{2} \cdot 2\right) \circ C_{3}$ and $\Pi(|G|)=\{2,3,5\}$. In any case $C_{G}\left(O_{3}(G)\right)$ is irreducible and fixes only one lattice $L$. In both cases, there exists some $F \in \mathcal{F}_{>0}\left(N_{i}\right)$ that is integral on $L$ with $\operatorname{det}(L, F)=1$.

- Let $C \simeq \mathbb{Q}(\sqrt{-2}, \sqrt{-3})$ be the commuting algebra of $N_{1}$ and let $K \simeq \mathbb{Q}(\sqrt{6})$ be its maximal totally real subfield. Since $\operatorname{End}_{\mathbb{Z} N}(N)$ is the maximal order in $C$ and since $\mathbb{Z}_{K,>0}^{*}=\operatorname{Nr}_{C / K}\left(\mathbb{Z}_{C}^{*}\right)$, we may restrict ourselves to one class of totally positive units. Let $\sigma \in \operatorname{Gal}(K / \mathbb{Q})$ such that $\sigma$ interchanges the two prime ideals over 5. One finds that $\sigma$ is conjugation by some $x \in N_{\mathrm{GL}_{16}(\mathbb{Q})}\left(N_{1}\right) \cap \mathrm{GL}(L)$. By Table 2.5.4. $G$ must be conjugate to $\operatorname{Aut}_{\mathbb{Q}(\sqrt{-d})}\left(L, \mathfrak{p}_{3}^{b}\left(\mathfrak{p}_{2} \mathfrak{p}_{5}\right)^{a} F\right)$ for some $a, b \in\{0,1\}$ and $d \in\{2,3\}$. The groups $\operatorname{Aut}_{\mathbb{Q}(\sqrt{-2})}(L, F) \simeq \sqrt{-2}\left[2_{+}^{1+6} \cdot\left(\mathrm{Alt}_{8}\right.\right.$ : $2)]_{8}$ and $\operatorname{Aut}_{\mathbb{Q}(\sqrt{-3})}(L, F) \simeq{ }_{\sqrt{-3}}\left[C_{6}\right]_{1} \otimes E_{8}$ are s.i.m.f., but have the wrong Fitting subgroups. The groups $\operatorname{Aut}_{\mathbb{Q}(\sqrt{-d})}\left(L, \mathfrak{p}_{3} F\right)$ are properly contained in $\operatorname{Aut}_{\mathbb{Q}(\sqrt{-d})}(L, F)$. Finally $\operatorname{Aut}_{\mathbb{Q}(\sqrt{-d})}\left(L, \mathfrak{p}_{2} \mathfrak{p}_{3}^{b} \mathfrak{p}_{5} F\right)=N_{1}$. So $C_{G}\left(O_{3}(G)\right) \not N_{1}$.

- Let $C \simeq \mathbb{Q}(\sqrt{2}, \sqrt{-3})$ be the commuting algebra of $N_{2}$ and let $K \simeq \mathbb{Q}(\sqrt{2})$ be its maximal totally real subfield. By Table 2.5.4, $G$ is conjugate to $\operatorname{Aut}_{\mathbb{Q}(\sqrt{-d})}\left(L, \mathfrak{p}_{2}^{a} F\right)$ for some $a \in\{0,1\}$ and $d \in\{3,6\}$. These groups are

\begin{tabular}{|c|c|c|}
\hline forms & $\mathbb{Q}(\sqrt{-3})$ & $\mathbb{Q}(\sqrt{-6})$ \\
\hline \hline$F$ & $\sqrt{-3}\left[\operatorname{Sp}_{4}(3) \circ C_{3}\right]_{4}^{2}$ & $\sqrt{-6}\left[\infty, 2\left[2_{-}^{1+4} \cdot \mathrm{Alt}_{5}\right]_{2} \stackrel{2(3)}{\otimes} A_{2}\right]_{8}$ \\
\hline $\mathfrak{p}_{2} F$ & $\infty, 2\left[2_{-}^{1+6} \cdot O_{6}^{-}(2)\right]_{4} \circ C_{3}$ & $\sqrt{-6}\left[\infty, 2\left[2_{-}^{1+4} \cdot \mathrm{Alt}_{5}\right]_{2} \underset{i}{2(3)} \infty, 3\left[\tilde{S}_{3}\right]_{1}\right]_{8}$ \\
\hline
\end{tabular}

This proves the claim. 
Lemma 4.9.23 If $O_{2}(G) \simeq 2_{+}^{1+4}$, then $G$ is conjugate to ${ }_{\sqrt{-6}}\left[F_{4} \stackrel{2}{\bigotimes} \underset{\infty, 3}{[}\left[\tilde{S}_{3}\right]_{1}\right]_{8}$ or $\sqrt{-6}\left[\left(F_{4} \otimes A_{2}\right): 2\right]_{8}$.

Proof: $G$ contains the normal subgroup $N:=\mathcal{B}^{\circ}\left(O_{2}(G)\right) O_{3}(G) \simeq{ }_{\sqrt{-3}}\left[C_{6}\right]_{1} \otimes F_{4}$ and $G / N \leq C_{2} \times C_{2}$. Thus $[G: N]=4$ by Lemma 2.2.1. So $G$ contains a subgroup of index two conjugate to $F_{4} \otimes{ }_{\infty, 3}\left[\tilde{S}_{3}\right]_{1}$ or $F_{4} \otimes A_{2}$. The result follows from Remark 2.2.17.

Lemma 4.9.24 $\mathrm{O}_{2}(G)$ is not conjugate to $D_{8} \otimes C_{4}$.

Proof: Otherwise $N:=\mathcal{B}^{\circ}(F(G)) \simeq{ }_{i}\left[\left(D_{8} \otimes C_{4}\right) \cdot S_{3}\right]_{2} \otimes C_{3}$ would be a normal subgroup of $G$. The centralizer of $N$ embeds into $\mathrm{GL}_{2}\left(\mathbb{Q}\left(\zeta_{12}\right)\right)$. Thus again $E(G)=1$ and $G / N \leq C_{2} \times C_{2}$. By Lemma 2.2.1 we get $[G: N]=4$. But MAGMA shows that no extension of $\mathrm{N}$ by $\mathrm{C}_{2} \times \mathrm{C}_{2}$ has the correct $\mathrm{O}_{2}$.

Lemma 4.9.25 $O_{2}(G)$ is not conjugate to $C_{8}, D_{16}, Q_{16}$ or $Q D_{16}$.

Proof: In any case $C_{G}(F(G))$ embeds into $\mathrm{GL}_{2}\left(\mathbb{Q}\left(\zeta_{24}\right)\right)$. So $E(G)=1$ by Table 2.5.1. Let $C=C_{G}\left(O_{3}(G)\right)$. Since $\operatorname{Out}(F(G))$ is a 2-group and $C / F(G)<\operatorname{Out}(F(G))$, we get $C=F(G)$. But then $[G: F(G)] \leq 2$ contradicts Lemma 2.2.1.

Lemma 4.9.26 If $O_{2}(G) \simeq Q_{8}$ then $G$ is conjugate to $i\left[\sqrt{-3}\left[\operatorname{Sp}_{4}(3) \circ C_{3}\right]_{4} \stackrel{2(2)}{\otimes} C_{4}\right]_{8}$ or $\left(\infty, 2\left[\mathrm{SL}_{2}(3)\right]_{1} \circ C_{3}\right) \otimes A_{4}$.

Proof: Let $B:=\mathcal{B}^{\circ}(F(G)) \simeq{ }_{\infty, 2}\left[\mathrm{SL}_{2}(3)\right]_{1} \circ C_{3}$. Then the centralizer $C_{G}(B)$ embeds into $\mathrm{GL}_{4}(\mathbb{Q}(\sqrt{-3}))$. If $E(G)=1$ then $G / B \leq C_{2} \times C_{2}$ shows that $G$ would be reducible. So it follows from Table 2.5.1, that $E(G)$ is conjugate to $\operatorname{Alt}_{5}, \sqrt{5}, \infty\left[\mathrm{SL}_{2}(5)\right]_{1}, \infty, 3\left[\mathrm{SL}_{2}(9)\right]_{2}$ or $\operatorname{Sp}_{4}(3)$. In any case, $G / F^{*}(G) \leq \operatorname{Out}\left(F^{*}(G)\right)$ implies $\Pi(|G|)=\{2,3,5\}$.

- If $E(G) \simeq \operatorname{Alt}_{5}$, then $N:=\mathcal{B}^{\circ}\left(F^{*}(G)\right) \simeq\left({ }_{\infty, 2}\left[\mathrm{SL}_{2}(3)\right]_{1} \circ C_{3}\right) \otimes A_{4}$ is a normal subgroup of $G$. But then $G=N$, since $N$ is already s.i.m.f., as we have seen before.

- If $E(G) \simeq \sqrt{5}, \infty\left[\mathrm{SL}_{2}(5)\right]_{1}$, then $B E(G)$ contains an irreducible cyclic subgroup of order 60. Theorem 4.9 .8 implies $G \simeq{ }_{\infty, 5}\left[\mathrm{SL}_{2}(5): 2\right]_{2} \underset{\sqrt{-3}}{\otimes}\left(\infty, 2\left[\mathrm{SL}_{2}(3)\right]_{1} \circ C_{3}\right)$.

- If $E(G) \simeq{ }_{\infty, 3}\left[\mathrm{SL}_{2}(9)\right]_{2}$ or $\mathrm{Sp}_{4}(3)$, then $G$ contains a subgroup $H$ conjugate to $\mathrm{SL}_{2}(5) \underset{\sqrt{-3}}{\otimes} C_{12}$ where the restriction of the natural character to $H$ is $4 \chi_{4}$. In particular $G$ contains an irreducible cyclic subgroup of order 60. Thus $G \simeq \sqrt{-3}\left[\mathrm{Sp}_{4}(3) \circ C_{3}\right]_{4} \underset{\sqrt{-3}}{\otimes} \infty, 2\left[\mathrm{SL}_{2}(3)\right]_{1}$ by Theorem 4.9.8. 


\section{Lemma 4.9.27}

(a) If $O_{2}(G) \simeq C_{4}$ then $G$ is conjugate to $i\left[\sqrt{-3}\left[\operatorname{Sp}_{4}(3) \circ C_{3}\right]_{4} \stackrel{2(2)}{\otimes} C_{4}\right]_{8}$.

(b) If $O_{2}(G) \simeq D_{8}$ then $G$ is conjugate to $\infty, 2\left[\mathrm{SL}_{2}(5) \stackrel{2(2)}{\otimes} D_{8}\right]_{4} \circ C_{3}$.

Proof: In both cases, $F(G)$ contains a characteristic subgroup $U$ isomorphic to $C_{12}$. If $E(G)=1$ then $G / F(G) \leq C_{2} \times C_{2}$ shows that $G$ would be reducible. So $E(G) \neq 1$ embeds into $\mathrm{GL}_{4}\left(\mathbb{Q}\left(\zeta_{12}\right)\right)$. By Table 2.5.1, this implies that $E(G)$ is conjugate to $\operatorname{Alt}_{5}, \quad \sqrt{5, \infty}\left[\mathrm{SL}_{2}(5)\right]_{1}, \infty, 3\left[\mathrm{SL}_{2}(9)\right]_{2}$ or $\operatorname{Sp}_{4}(3)$. In any case $G / F^{*}(G) \leq \operatorname{Out}\left(F^{*}(G)\right)$ shows that $\Pi(|G|)=\{2,3,5\}$.

- If $E(G) \simeq \operatorname{Alt}_{5}$, then $N:=\mathcal{B}^{\circ}(E(G) U) \simeq C_{12} \otimes A_{4}$ is irreducible with $C \simeq \mathbb{Q}\left(\zeta_{12}\right)$ as commuting algebra. It fixes 4 lattices but there exists a $N$-normal critical set $\left\{L_{1}, L_{2}\right\}$. Since $N$ is not normal in $\operatorname{Aut}_{\mathbb{Q}\left(\zeta_{12}\right)}\left(L_{1}, F\right) \simeq_{i}\left[\left(2_{+}^{1+4} \otimes C_{4}\right) . S_{6}\right]_{4} \otimes C_{3}$ for some $F \in \mathcal{F}_{>0}(N)$, it follows that $G$ must fix $L_{2}$. Clearly $\Pi(|G|)=\{2,3,5\}$ and one finds some $F \in \mathcal{F}_{>0}(N)$ that is integral on $L_{2}$ with $\operatorname{det}\left(L_{2}, F\right)=2^{8} \cdot 5^{4}$. Since $\operatorname{End}_{\mathbb{Z} N}\left(L_{2}\right)$ is the maximal order of $C$ and $\mathbb{Z}[\sqrt{3}]_{>0}^{*}=\operatorname{Nr}_{C / \mathbb{Q}(\sqrt{3})}\left(\mathbb{Z}_{C}^{*}\right)$ we may restrict ourselves to one class of totally positive units. By Table 2.5.4, this leaves the following four groups:

\begin{tabular}{|c|c|c|}
\hline lattice and form & $\mathbb{Q}(i)$ & $\mathbb{Q}(\sqrt{-3})$ \\
\hline \hline$\left(L_{2}, F\right)$ & $\varlimsup_{i}\left[\left(2_{+}^{1+6} \otimes C_{4}\right) \cdot \mathrm{Sp}_{6}(2)\right]_{8}$ & $\left({ }_{\infty, 2}\left[\mathrm{SL}_{2}(3)\right]_{1} \circ C_{3}\right) \otimes A_{4}$ \\
\hline$\left(L_{2}, \mathfrak{p}_{2}^{-1} \mathfrak{p}_{3} F\right)$ & $\lesseqgtr_{i}\left[\left(2_{+}^{1+4} \otimes C_{4}\right) \cdot S_{6}\right]_{4} \otimes A_{2}$ & $\left(\sqrt{-3}\left[C_{6}\right]_{1} \otimes A_{4}\right)^{2}$ \\
\hline
\end{tabular}

None has the correct generalized Fitting subgroup.

- If $E(G) \simeq \sqrt{5, \infty}\left[\mathrm{SL}_{2}(5)\right]_{1}$, then $U E(G)$ contains an irreducible cyclic subgroup of order 60. Theorem 4.9 .8 shows that $G \simeq \simeq_{\infty, 2}\left[\mathrm{SL}_{2}(5)^{2(2)} \otimes D_{8}\right]_{4} \circ C_{3}$.

- If $E(G) \simeq{ }_{\infty, 3}\left[\mathrm{SL}_{2}(9)\right]_{2}$ or $\operatorname{Sp}_{4}(3)$, then $G$ contains a subgroup $H$ conjugate to $\mathrm{SL}_{2}(5) \underset{\sqrt{-3}}{\otimes} C_{12}$ where the restriction of the natural character to $\mathrm{SL}_{2}(5)$ is $4 \chi_{4}$. In particular $G$ contains an irreducible cyclic subgroup of order 60 . Thus $G$ is conjugate to $i\left[\sqrt{-3}\left[\mathrm{Sp}_{4}(3) \circ C_{3}\right]_{4}^{2(2)} \underset{\otimes}{\otimes} C_{4}\right]_{8}$ by Theorem 4.9 .8 .

Lemma 4.9.28 If $\mathrm{O}_{2}(G)=C_{2}$, then $G$ is conjugate to one of $\sqrt{-3}\left[C_{6}\right]_{1} \otimes E_{8}$,

$$
\begin{gathered}
\sqrt{-7}\left[2 . \mathrm{Alt}_{7}\right]_{4} \underset{\sqrt{-7}}{\otimes} \infty, 3\left[\tilde{S}_{3}\right]_{1}, \underset{\sqrt{-7}}{ }\left[2 . \mathrm{Alt}_{7}\right]_{4} \otimes A_{2}, \sqrt{-3}\left[C_{6}\right]_{1} \otimes\left[\left(\mathrm{SL}_{2}(5) \stackrel{2}{\square} \mathrm{SL}_{2}(5)\right): 2\right]_{8}, \\
\sqrt{-15}\left[\left(\left(\mathrm{SL}_{2}(5) \circ \mathrm{SL}_{2}(5)\right): 2\right) \stackrel{2_{+}}{\otimes} \underset{\sqrt{-3}}{ }\left[C_{6}\right]_{1}\right]_{8}, \sqrt{-15}\left[\left(\left(\mathrm{SL}_{2}(5) \circ \mathrm{SL}_{2}(5)\right): 2\right) \stackrel{2}{\otimes}_{\sqrt{-3}}\left[C_{6}\right]_{1}\right]_{8} .
\end{gathered}
$$

Proof: Let $N:=\mathcal{B}^{\circ}\left(F^{*}(G)\right) \unlhd G$. By Table 2.5.1 there are the following possibilities for $E(G)$ : 
- $E(G) \simeq \mathrm{Alt}_{5}$. Then $N \simeq{ }_{\sqrt{-3}}\left[C_{6}\right]_{1} \otimes A_{4}$ has $\mathbb{Q}(\sqrt{-3})$ as commuting algebra and $[G: N] \leq 2$. This contradicts Lemma 2.2.1.

- $E(G) \simeq \sqrt{5}, \infty\left[\mathrm{SL}_{2}(5)\right]_{1}$. Let $C:=C_{G}\left(O_{3}(G)\right)$. It follows from Lemma 2.2.1 that $[G: C]=[C: N]=2$. Thus $C$ is reducible and conjugate to $\infty, 5\left[\mathrm{SL}_{2}(5): 2\right]_{2} \circ C_{3}$ or $\infty, 5\left[\mathrm{SL}_{2}(5) \cdot 2\right]_{2} \circ C_{3}$. This contradicts loc. cit..

- $E(G) \simeq \mathrm{SL}_{2}(5) \circ \mathrm{SL}_{2}(5)$. Then $N=\left(\left(\mathrm{SL}_{2}(5) \circ \mathrm{SL}_{2}(5)\right): 2\right) \otimes C_{3}$ is irreducible, has $\mathbb{Q}(\sqrt{5}, \sqrt{-3})$ as commuting algebra and fixes only one lattice $L$. One finds some $F \in \mathcal{F}_{>0}(N)$ that is integral on $L$ with $\operatorname{det}(L, F)=3^{8}$. Further $G / F^{*}(G) \leq$ $\operatorname{Out}\left(F^{*}(G)\right) \simeq D_{8} \times C_{2}$ shows $\Pi(|G|)=\{2,3,5\}$. By Table 2.5.4, we have to check the following groups:

\begin{tabular}{|c|c|c|}
\hline & $\mathbb{Q}(\sqrt{-3})$ & $\mathbb{Q}(\sqrt{-15})$ \\
\hline \hline$F$ & $\sqrt{-3}\left[C_{6}\right]_{1} \otimes E_{8}$ & $\left.\sqrt{-15}\left[\left(\mathrm{SL}_{2}(5) \circ \mathrm{SL}_{2}(5)\right): 2\right) \stackrel{2}{\otimes}_{\sqrt{-3}}\left[C_{6}\right]_{1}\right]_{8}$ \\
\hline $\mathfrak{p}_{5} F$ & $\sqrt{-3}\left[C_{6}\right]_{1} \otimes\left[\left(\mathrm{SL}_{2}(5) \stackrel{2}{\square} \mathrm{SL}_{2}(5)\right): 2\right]_{8}$ & $\left.\sqrt{-15}\left[\left(\mathrm{SL}_{2}(5) \circ \mathrm{SL}_{2}(5)\right): 2\right) \stackrel{\otimes}{\sqrt{-3}}_{\sqrt{2}}\left[C_{6}\right]_{1}\right]_{8}$ \\
\hline
\end{tabular}

All groups except $\sqrt{-3}\left[C_{6}\right]_{1} \otimes E_{8}$ have the correct generalized Fitting subgroups.

- $E(G) \simeq \mathrm{L}_{2}(7)$. Then $N=M_{8,3} \otimes C_{3}$ is irreducible with commuting algebra $\mathbb{Q}(\sqrt{-3})$ and fixes 12 lattices. By computing the corresponding automorphism groups, one checks that $N$ is only contained in $\sqrt{-3}\left[C_{6}\right]_{1}^{8}$.

- $E(G) \simeq{ }_{\infty, 3}\left[\mathrm{SL}_{2}(7)\right]_{4}$ contradicts Lemma 4.9.3.

- $E(G) \simeq \mathrm{SL}_{2}(9)$. Then $E(G) \not \mathcal{B}^{o}(N) \simeq \sqrt{-3}\left[\mathrm{Sp}_{4}(3) \circ C_{3}\right]_{4}$ (see Lemma 4.5.6).

- $E(G) \simeq{ }_{\sqrt{-7}}\left[2 . \mathrm{Alt}_{7}\right]_{4}$. Then $N={ }_{\sqrt{-7}}\left[2 . \mathrm{Alt}_{7}\right]_{4} \otimes_{\sqrt{-3}}\left[C_{6}\right]_{1}$ is irreducible with commuting algebra $C \simeq \mathbb{Q}(\sqrt{-3}, \sqrt{-7})$ and fixes one lattice $L$. Again one concludes $\Pi(|G|)=\{2,3,5,7\}$. Further there exists some $F \in \mathcal{F}_{>0}(N)$ that is integral on $L$ with $\operatorname{det}(L, F)=3^{8}$. Let $K \simeq \mathbb{Q}(\sqrt{21})$ be the maximal totally real subfield of $C$. Since $\operatorname{End}_{\mathbb{Z} N}(L)$ is the maximal order of $C$ and since $\mathbb{Z}_{K,>0}^{*}=\operatorname{Nr}_{C / K}\left(\mathbb{Z}_{C}^{*}\right)$, we may restrict ourselves to one class of totally positive units. Let $\sigma \in \operatorname{Gal}(K / \mathbb{Q})$ such that $\sigma$ interchanges the two prime ideals over 5. One finds that $\sigma$ is conjugation by some $x \in N_{\mathrm{GL}_{16}(\mathbb{Q})}(N) \cap \mathrm{GL}(L)$ such that $x F x^{\operatorname{tr}}=F$. Thus by Table 2.5.4. $G$ is conjugate to $\operatorname{Aut}_{\mathbb{Q}(\sqrt{-d})}\left(L, \mathfrak{p}_{3}^{-a} \mathfrak{p}_{5}^{a} \mathfrak{p}_{7}^{b} F\right)$ for some $a, b \in\{0,1\}$ and $d \in\{3,7\}$. If $a=1$ then these groups equal $N$. The other four groups are:

\begin{tabular}{|c|c|c|}
\hline form & $\mathbb{Q}(\sqrt{-3})$ & $\mathbb{Q}(\sqrt{-7})$ \\
\hline \hline$F$ & $\sqrt{-3}\left[C_{6}\right]_{1} \otimes E_{8}$ & $\sqrt{-7}\left[2 . \mathrm{Alt}_{7}\right]_{4} \otimes A_{2}$ \\
\hline $\mathfrak{p}_{7} F$ & $\lessgtr \sqrt{-3}\left[C_{6}\right]_{1} \otimes E_{8}$ & $\sqrt{-7}\left[2 . \mathrm{Alt}_{7}\right]_{4} \underset{\sqrt{-7}}{\otimes} \infty, 3\left[\tilde{S}_{3}\right]_{1}$ \\
\hline
\end{tabular}

So $G$ is conjugate to $\sqrt{-7}\left[2 . \mathrm{Alt}_{7}\right]_{4} \otimes A_{2}$ or $\sqrt{-7}\left[2 . \mathrm{Alt}_{7}\right]_{4} \underset{\sqrt{-7}}{\otimes} \infty, 3\left[\tilde{S}_{3}\right]_{1}$.

- $E(G) \simeq \mathrm{SL}_{2}(7)$ with character $2 \chi_{4 a b}$. The commuting algebra $C$ of $N$ is isomorphic to $\mathbb{Q}(\sqrt{-3}, \sqrt{-7})$ with $K \simeq \mathbb{Q}(\sqrt{21})$ as maximal totally real subfield. Then 
$N$ fixes 4 lattices that have $\mathbb{Z}_{C}$ as endomorphism ring but only one class has minimal $N$-invariant superlattices whose endomorphism ring is a proper suborder of $\mathbb{Z}_{C}$. So by Remark 2.2 .8 this yields a $N$-normal critical lattice $L$. Further there exists some $F \in \mathcal{F}_{>0}(N)$ that is integral on $L$ with $\operatorname{det}(L, F)=2^{4} \cdot 3^{8}$. Again we have $\Pi(|G|)=\{2,3,7\}$. Just as before we may restrict ourselves to one class of totally positive units. By Table 2.5.4. $G$ must be conjugate to $\operatorname{Aut}_{\mathbb{Q}(\sqrt{-d})}\left(L, \mathfrak{p}_{7}^{a} F\right)$ for some $a \in\{0,1\}$ and $d \in\{3,7\}$. One checks that these automorphism groups are conjugate to $N$ if $a=1$. And they yield proper subgroups of $\sqrt{-3}\left[C_{6}\right]_{1}^{8}$ and $\sqrt{-7}\left[2 . \mathrm{Alt}_{7}\right]_{4} \otimes A_{2}$ if $a=0$. So this case never happens.

- $E(G) \simeq \operatorname{Sp}_{4}(3)$. Then $N \simeq{ }_{\sqrt{-3}}\left[\operatorname{Sp}_{4}(3) \circ C_{3}\right]_{4}$ is reducible. Again by Lemma 2.2.1 we conclude $[G: N]=4$. Thus $G$ contains a subgroup of index two conjugate to $\operatorname{Sp}_{4}(3) \underset{\sqrt{-3}}{\otimes} \infty, 3\left[\tilde{S}_{3}\right]_{1}$ or $\operatorname{Sp}_{4}(3) \otimes A_{2}$. Both groups have $\mathbb{Q}(\sqrt{-3})$ as commuting algebras and fix one or three lattices respectively. One checks that they are only contained in $\sqrt{-3}\left[\operatorname{Sp}_{4}(3) \circ C_{3}\right]_{4}^{2}$.

- $E(G) \simeq \operatorname{Aut}\left(E_{8}\right)=2 . O_{8}^{+}(2) .2$. Then $N={ }_{\sqrt{-3}}\left[C_{6}\right]_{1} \otimes E_{8}$ is already s.i.m.f..

\subsubsection{The case $O_{p}(G)=1$ for all odd primes $p$}

Let $G<\operatorname{Sp}_{16}(\mathbb{Q})$ be s.p.i.m.f. such that $F(G)=O_{2}(G)$. It follows from Table 2.5 .2 that $O_{2}(G)$ is isomorphic to $C_{2}, C_{4}, D_{8}, Q_{8}, C_{8}, D_{8} \otimes C_{4}, D_{16}, Q_{16}, Q D_{16}, C_{16}$, $D_{8} \otimes C_{8}, D_{8} \otimes D_{16}, D_{8} \otimes Q_{16}, D_{8} \otimes Q D_{16}, 2_{+}^{1+4}, 2_{+}^{1+4} \otimes C_{4}, 2_{-}^{1+4}, C_{32}, D_{32}, Q_{32}, Q D_{32}$, $Q D_{64}, D_{8} \otimes C_{16}, D_{8} \otimes Q D_{32}, D_{8} \otimes D_{16}, 2_{+}^{1+4} \otimes C_{8}, 2_{+}^{1+4} \otimes D_{16}, 2_{+}^{1+4} \otimes Q D_{16}, 2_{-}^{1+6}, 2_{+}^{1+6}$ or $2_{-}^{1+6} \otimes C_{4}$.

We discuss each possibility, which finishes the classification of the s.p.i.m.f. subgroups of $\operatorname{Sp}_{16}(\mathbb{Q})$.

\section{Lemma 4.9.29}

(a) If $F(G) \simeq 2_{+}^{1+6}$ then $G$ is conjugate to $\sqrt{-2}\left[2_{+}^{1+6} \cdot\left(\operatorname{Alt}_{8}: 2\right)\right]_{8}$.

(b) If $F(G) \simeq 2_{+}^{1+6} \otimes C_{4}$ then $G$ is conjugate to ${ }_{i}\left[\left(2_{+}^{1+6} \otimes C_{4}\right) \cdot \operatorname{Sp}_{6}(2)\right]_{8}$.

(c) If $F(G) \simeq 2_{-}^{1+6}$ then $G$ is conjugate to $\sqrt{-2}\left[\infty, 2\left[2_{-}^{1+6} \cdot O_{6}^{-}(2)\right]_{4}: 2\right]_{8}$.

Proof:

(a) Follows from Lemma 3.4.3.

(b) In this case $\mathcal{B}^{\circ}(F(G)) \simeq{ }_{i}\left[\left(2_{+}^{1+6} \otimes C_{4}\right) \cdot \operatorname{Sp}_{6}(2)\right]_{8}$ fixes only one lattice and has $\mathbb{Q}(i)$ as commuting algebra. One checks that it is s.i.m.f..

(c) Follows from Lemma 4.9.4. 
Lemma 4.9.30 $F(G)$ is not conjugate to $2_{+}^{1+4} \otimes C_{8}, 2_{+}^{1+4} \otimes D_{16}$ or $2_{+}^{1+4} \otimes Q D_{16}$.

Proof: In any case $G$ would contain an irreducible normal subgroup $N$ conjugate to $2_{+}^{1+4} \otimes C_{8}$. Thus $G$ contains a normal subgroup $B:=\mathcal{B}^{\circ}(N) \simeq N . S_{6}$. This group fixes up to isomorphism only one lattice $L$ and its commuting algebra is isomorphic to $\mathbb{Q}\left(\zeta_{8}\right)$ with maximal totally real subfield $\mathbb{Q}(\sqrt{2})$. Thus $B$ is self-centralizing and from the structure of $\operatorname{Out}(N)$ we get $G / B \leq C_{2} \times C_{2}$. Hence $\Pi(|G|)=\{2,3,5\}$. There exist some $F \in \mathcal{F}_{>0}(B)$ that is integral on $L$ with $\operatorname{det}(L, F)=1$. By Table 2.5.4, $G$ is conjugate to $\operatorname{Aut}_{\mathbb{Q}(\sqrt{-d})}\left(L, \mathfrak{p}_{2}^{a} F\right)$ for some $a \in\{0,1\}$ and $d \in\{1,2\}$. These groups are:

\begin{tabular}{|c|c|c|}
\hline form & $\mathbb{Q}(i)$ & $\mathbb{Q}(\sqrt{-2})$ \\
\hline \hline$F$ & ${ }_{i}\left[\left(2_{+}^{1+4} \otimes C_{4}\right) \cdot S_{6}\right]_{4}^{2}$ & $\sqrt{-2}\left[2_{+}^{1+6} \cdot\left(\mathrm{Alt}_{8}: 2\right)\right]_{8}$ \\
\hline $\mathfrak{p}_{2} F$ & ${ }_{i}\left[\left(2_{+}^{1+6} \otimes C_{4}\right) \cdot \mathrm{Sp}_{6}(2)\right]_{8}$ & $\sqrt{-2}\left[{ }_{\infty, 2}\left[2_{-}^{1+6} \cdot O_{6}^{-}(2)\right]_{4}: 2\right]_{8}$ \\
\hline
\end{tabular}

None of these groups has the correct Fitting subgroup.

\section{Lemma 4.9.31}

(a) If $F(G) \simeq D_{8} \otimes Q D_{32}$ then $G$ is conjugate to ${ }_{\zeta_{16}-\zeta_{16}^{-1}}\left[\left(D_{8} \otimes Q D_{32}\right) . S_{3}\right]_{4}$.

(b) $F(G)$ is not conjugate to $D_{8} \otimes C_{16}$ or $D_{8} \otimes D_{16}$.

Proof: In any case, $G$ contains an irreducible normal subgroup $N$ conjugate to $D_{8} \otimes C_{16}$. Thus $G$ contains a normal subgroup $B:=\mathcal{B}^{\circ}(N) \simeq N . S_{3}$. This group fixes only one lattice $L$ and its commuting algebra is isomorphic to $\mathbb{Q}\left(\zeta_{16}\right)$. Thus $B$ is self-centralizing and from the structure of $\operatorname{Out}(N)$ we get $G / B \leq C_{2} \times C_{4}$. Hence $\Pi(|G|)=\{2,3\}$. Let $F \in \mathcal{F}_{>0}(B)$ such that $F$ is integral on $L$ with $\operatorname{det}(L, F)=1$. By Table 2.5.3, $G$ is conjugate to $\operatorname{Aut}_{\mathbb{Q}(\alpha)}\left(L, \mathfrak{p}_{2}^{a} F\right)$ for some $0 \leq a \leq 2$ and $\alpha \in\left\{i, \sqrt{-2}, \zeta_{16}-\zeta_{16}^{-1}\right\}$. These groups are

\begin{tabular}{|c|c|c|c|}
\hline form & $\mathbb{Q}(i)$ & $\mathbb{Q}(\sqrt{-2})$ & $\mathbb{Q}\left(\zeta_{16}-\zeta_{16}^{-1}\right)$ \\
\hline \hline$F$ & ${ }_{i}\left[\left(2_{+}^{1+4} \otimes C_{4}\right) \cdot S_{6}\right]_{4}^{2}$ & $\sqrt{-2}\left[\infty, 2\left[\left(D_{8} \otimes Q_{8}\right) \cdot \mathrm{Alt}_{5}\right]_{2}: 2\right]_{4}^{2}$ & $\zeta_{16}-\zeta_{16}^{-1}\left[\left(D_{8} \otimes Q D_{32}\right) \cdot S_{3}\right]_{4}$ \\
\hline $\mathfrak{p}_{2} F$ & $\zeta_{i}\left[\left(D_{8} \otimes C_{4}\right) \cdot S_{3}\right]_{2}^{4}$ & $\lesseqgtr \sqrt{-2}\left[F_{4}: 2\right]_{4}^{2}$ & $\zeta_{16}-\zeta_{16}^{-1}\left[\left(D_{8} \otimes Q D_{32}\right) \cdot S_{3}\right]_{4}$ \\
\hline $\mathfrak{p}_{2}^{2} F$ & ${ }_{i}\left[\left(D_{8} \otimes C_{4}\right) \cdot S_{3}\right]_{2}^{4}$ & $\sqrt{-2}\left[F_{4}: 2\right]_{4}^{2}$ & $\zeta_{16}-\zeta_{16}^{-1}\left[\left(D_{8} \otimes Q D_{32}\right) \cdot S_{3}\right]_{4}$ \\
\hline
\end{tabular}

So the result follows.

Lemma 4.9.32 $F(G)$ is not conjugate to $C_{16}, D_{32}$ or $Q D_{32}$.

Proof: Otherwise $C_{G}(F(G))$ embeds into $\mathrm{GL}_{2}\left(\mathbb{Q}\left(\zeta_{16}\right)\right)$, so $E(G)=1$. Thus $G / F(G) \leq$ $\operatorname{Out}(F(G))$ is a 2-group, which implies $G=F(G)$. But then $G$ is reducible. 


\section{Lemma 4.9.33}

(a) If $F(G) \simeq_{\zeta_{32}-\zeta_{32}^{-1}}\left[Q D_{64}\right]_{2}$ then $G=F(G)$ is s.i.m.f..

(b) $F(G)$ is not conjugate to $Q_{32}$ or $C_{32}$.

Proof: Clearly $E(G)=1$ and thus $F(G)$ is self-centralizing in $G$. Since $\operatorname{Out}(F(G))$ is a 2-group, we have $G=F(G)$. The group $\zeta_{32-\zeta_{32}^{-1}}\left[Q D_{64}\right]_{2}$ is s.i.m.f. by Lemma 3.3 .1 whereas $C_{32} \lesseqgtr Q D_{64}$ and $Q_{32} \lesseqgtr Q_{32} \circ C_{3}$.

Lemma 4.9.34 $F(G)$ is not conjugate to $2_{-}^{1+4}$.

Proof: If $E(G)=1$, then $B:=\mathcal{B}^{\circ}(F(G)) \simeq{ }_{\infty, 2}\left[2_{-}^{1+4} \text {. Alt }\right]_{5}$ and $G / B \leq C_{2}$ contradicts Lemma 2.2.1. So $E(G) \neq 1$ embeds into $\mathrm{GL}_{2}\left(\mathcal{Q}_{\infty, 2}\right)$ and thus must be conjugate to $\sqrt{5, \infty}\left[\mathrm{SL}_{2}(5)\right]_{1}$ by Table 2.5.1. The group $\sqrt{5}, \infty\left[\mathrm{SL}_{2}(5)\right]_{1} \underset{\infty, 2}{\otimes}, 2\left[2_{-}^{1+4} \cdot \text { Alt }_{5}\right]_{2}$ is irreducible and has $\mathbb{Q}(\sqrt{5})$ as commuting algebra. In this case $G$ would not be symplectic.

Lemma 4.9.35 $F(G)$ is not conjugate to $2_{+}^{1+4} \otimes C_{4}$.

Proof: The centralizer $C_{G}(F(G))$ embeds into $\mathrm{GL}_{2}(\mathbb{Q}(i))$. So it follows from Table 2.5.1. that $E(G)=1$. Thus $G$ contains $B:=\mathcal{B}^{\circ}(F(G)) \simeq F(G) . S_{6}$ of index at most 2 and $B$ has $\mathbb{Q}(i)^{2 \times 2}$ as commuting algebra. This contradicts Lemma 2.2.1.

Lemma 4.9.36 $F(G)$ is not conjugate to $2_{+}^{1+4}$.

Proof: Otherwise $G$ contains the normal subgroup $N:=\mathcal{B}^{\circ}(F(G)) \simeq \operatorname{Aut}\left(F_{4}\right)$. Then $C_{G}(N)$ embeds into $\mathrm{GL}_{4}(\mathbb{Q})$. Table 2.5.1 implies that $E(G)=1$ (note that $F_{4} \otimes A_{4}$ is not symplectic). But then $G / N \leq \operatorname{Out}(N) \simeq C_{2}$ implies that $G$ is reducible.

Lemma 4.9.37 $F(G)$ is not conjugate to $D_{8} \otimes C_{8}, D_{8} \otimes D_{16}, D_{8} \otimes Q_{16}$ or $D_{8} \otimes Q D_{16}$.

Proof: In any cases $E(G)=1$ and $G$ would contain a normal subgroup $N$ conjugate to $D_{8} \otimes C_{8}=Q_{8} \otimes C_{i} C_{8}$. Let $B:=\mathcal{B}^{\circ}(N) \simeq{ }_{\infty, 2}\left[\mathrm{SL}_{2}(3)\right]_{1} \underset{i}{\otimes} C_{8}$. It follows from $G / F(G) \leq$ $\operatorname{Out}(F(G))$ that $G$ is soluble and $|G|=2^{k} \cdot 3$ for some $k>0$. Moreover $B$ contains a characteristic subgroup $U$ conjugate to $\infty, 2\left[\mathrm{SL}_{2}(3)\right]_{1}$. But $C_{G}(U)$ is necessarily a 2-group, so $C_{G}(U) \subseteq F(G)$. Hence $G$ contains $U F(G)$ as a normal subgroup of index at most 2. Now Lemma 2.2.1 implies $F(G) \simeq D_{8} \otimes Q_{16}$ or $D_{8} \otimes D_{16}$. In both cases, $\mathcal{B}^{o}(F(G)) \simeq F(G) . S_{3}$ implies that $\mathcal{B}^{o}(F(G))=G$ since $\left|\mathcal{B}^{o}(F(G))\right|=2 \cdot|U F(G)|$.

If $F(G) \simeq D_{8} \otimes Q_{16}$, then $F(G)$ has $\mathcal{Q}_{\sqrt{2}, \infty, \infty}$ as commuting algebra which contains nontrivial torsion units. So $G$ cannot be s.i.m.f..

If $F(G) \simeq D_{8} \otimes D_{16}=Q_{16} \circ Q_{8}$ then $\mathcal{B}^{\circ}(F(G)) \simeq \sqrt{2}, \infty\left[Q_{16}\right]_{1}{ }_{\infty, 2}\left[\mathrm{SL}_{2}(3)\right]_{1}$ is reducible. $\square$ 
Lemma 4.9.38 If $F(G) \simeq D_{8} \otimes C_{4}$ then $G$ is conjugate to

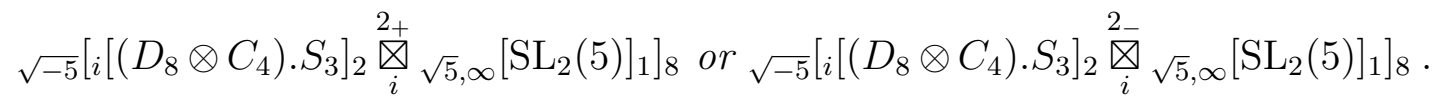

Proof: Let $B:=\mathcal{B}^{\circ}(F(G)) \simeq_{i}\left[\left(D_{8} \otimes C_{4}\right) . S_{3}\right]_{2}$. Then $C_{G}(B)$ embeds into $\mathrm{GL}_{4}(\mathbb{Q}(i))$. By 2.5.1 $E(G)$ is conjugate to $1, \operatorname{Alt}_{5}, \sqrt{5}, \infty\left[\mathrm{SL}_{2}(5)\right]_{1}$ or ${ }_{\infty, 3}\left[\mathrm{SL}_{2}(9)\right]_{2}$. Let $N:=B E(G) \unlhd$ $G$.

- If $E(G)=1$, then $[G: B] \leq 2$. This contradicts Lemma 2.2.1.

- If $E(G) \simeq \mathrm{Alt}_{5}$ or ${ }_{\infty, 3}\left[\mathrm{SL}_{2}(9)\right]_{2}$, then $N$ is already irreducible, fixes 4 or 2 lattices respectively and has $\mathbb{Q}(i)$ as commuting algebra. It is easily checked that $N$ is only contained in ${ }_{i}\left[\left(2_{+}^{1+6} \otimes C_{4}\right) \cdot \operatorname{Sp}_{6}(2)\right]_{8}$.

- If $E(G) \simeq \sqrt{5, \infty}\left[\mathrm{SL}_{2}(5)\right]_{1}$ then $N$ contains an irreducible cyclic subgroup of order 40. Since $\operatorname{Out}(N)$ is a 2 -group, we have $\Pi(|G|)=\{2,3,5\}$. Hence $G$ is conjugate

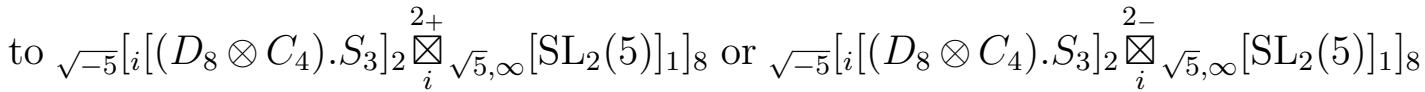
by Theorem 4.9.6.

Thus the claim follows.

Lemma 4.9.39 $F(G)$ is not conjugate to $C_{8}, D_{16}$ or $Q D_{16}$.

Proof: If $E(G)=1$, then $G / F(G) \leq \operatorname{Out}(F(G))$ is a 2-group. Which implies that $G=F(G)$ is reducible. In any case, $F(G)$ contains a characteristic subgroup $H \simeq C_{8}$. Thus $E(G)$ embeds into $\mathrm{GL}_{4}\left(\mathbb{Q}\left(\zeta_{8}\right)\right)$. So $E(G) \neq 1$ is conjugate to $\mathrm{Alt}_{5}, \sqrt{5}, \infty\left[\mathrm{SL}_{2}(5)\right]_{1}$ or $\infty, 3\left[\mathrm{SL}_{2}(9)\right]_{2}$. In any case, $G / F^{*}(G) \leq \operatorname{Out}(F(G))$ shows that $\Pi(|G|)=\{2,3,5\}$. Moreover $H \mathcal{B}^{\circ}(E(G)) \unlhd G$ contains an irreducible cyclic subgroup of order 40 . Thus the result follows from Theorem 4.9.6.

Lemma 4.9.40 If $F(G) \simeq Q_{8}$ then $G$ is conjugate to one of

$$
\begin{aligned}
& \sqrt{-10}\left[\sqrt{-2}\left[\mathrm{GL}_{2}(3)\right]_{2} \underset{\sqrt{-2}}{\stackrel{2}{\otimes}} \sqrt{5}, \infty\left[\mathrm{SL}_{2}(5)\right]_{1}\right]_{8}, \sqrt{-10}\left[\sqrt{-2}\left[\mathrm{GL}_{2}(3)\right]_{2} \underset{\sqrt{-2}}{\stackrel{2_{+}}{\otimes}} \sqrt{5}, \infty\left[\mathrm{SL}_{2}(5)\right]_{1}\right]_{8} \\
& \sqrt{-2}\left[\mathrm{GL}_{2}(3)\right]_{2} \underset{\sqrt{-2}}{\otimes} \infty\left[\mathrm{SL}_{2}(5): 2\right]_{2}, \sqrt{-2}\left[\mathrm{GL}_{2}(3)\right]_{2} \otimes A_{4}, \infty, 2\left[\mathrm{SL}_{2}(3)\right]_{1} \underset{i}{\stackrel{2}{\otimes}}{ }_{\infty, 3}\left[\mathrm{SL}_{2}(9)\right]_{2} \text {. }
\end{aligned}
$$

Proof: The layer $E(G)$ embeds into $\mathrm{GL}_{4}\left(\mathcal{Q}_{\infty, 2}\right)$. Since $\operatorname{Out}\left(\mathcal{B}^{\circ}\left(Q_{8}\right)\right) \simeq C_{2}, E(G)$ cannot be trivial. Table 2.5.1 shows that $E(G)$ is conjugate to $\operatorname{Alt}_{5}, \sqrt{5}, \infty\left[\mathrm{SL}_{2}(5)\right]_{1}$ or ${ }_{\infty, 3}\left[\mathrm{SL}_{2}(9)\right]_{2}$ (note that $\left.\mathrm{O}_{3}\left(\mathcal{B}^{\circ}\left(\mathrm{Sp}_{4}(3)\right)\right) \simeq C_{3}\right)$.

- If $E(G) \simeq$ Alt $_{5}$ then $\mathcal{B}^{o}\left(F^{*}(G)\right) \simeq \infty_{\infty, 2}\left[\mathrm{SL}_{2}(3)\right]_{1} \otimes A_{4}$ is an irreducible normal subgroup of $G$ and it has $\mathcal{Q}_{\infty, 2}$ as commuting algebra. By Remark 2.2.17, $G$ is conjugate to $\sqrt{-2}\left[\mathrm{GL}_{2}(3)\right]_{2} \otimes A_{4}$. This group is s.i.m.f.. 
- If $E(G) \simeq \sqrt{5, \infty}\left[\mathrm{SL}_{2}(5)\right]_{1}$, then $G$ contains a normal subgroup $N:=\mathcal{B}^{o}\left(F^{*}(G)\right)$ conjugate to $\sqrt{5}, \infty\left[\mathrm{SL}_{2}(5)\right]_{1} \circ \mathrm{SL}_{2}(3)$ and $G / N \leq \operatorname{Out}(N) \simeq C_{2} \times C_{2}$.

First we handle the case that $[G: N]=2$. The groups $\sqrt{5}, \infty\left[\mathrm{SL}_{2}(5)\right]_{1}{ }_{\square}^{2} \mathrm{SL}_{2}(3)$ and $\sqrt{5}, \infty\left[\mathrm{SL}_{2}(5)\right]_{1} \square \mathrm{SL}_{2}(3)$ are reducible.

The groups $\infty, 5\left[\mathrm{SL}_{2}(5): 2\right]_{2} \underset{\sqrt{-2}}{\otimes} \infty, 2\left[\mathrm{SL}_{2}(3)\right]_{1}$ and $\infty, 5\left[\mathrm{SL}_{2}(5) \cdot 2\right]_{2} \underset{\sqrt{-2}}{\otimes} \infty, 2\left[\mathrm{SL}_{2}(3)\right]_{1}$ are proper subgroups of $\sqrt{-2}\left[\mathrm{GL}_{2}(3)\right]_{2} \underset{\sqrt{-2}}{\otimes} \infty, 5\left[\mathrm{SL}_{2}(5): 2\right]_{2}$ and $\sqrt{--2}_{+}\left[2^{1+6}\right.$.(Alt $\left.\left.8: 2\right)\right]_{8}$ respectively. The group $\sqrt{5}, \infty\left[\mathrm{SL}_{2}(5)\right]_{1} \underset{\infty, 2}{\otimes} \sqrt{2}, \infty\left[\tilde{S}_{4}\right]_{1}$ is not symplectic. Finally $H:={ }_{5}, \infty\left[\mathrm{SL}_{2}(5)\right]_{1} \underset{\sqrt{-2}}{\otimes} \sqrt{-2}\left[\mathrm{GL}_{2}(3)\right]_{1}$ is irreducible, has $\mathbb{Q}(\sqrt{-2}, \sqrt{5})$ as commuting algebra and fixes only one lattice $L$. There exists some $F \in \mathcal{F}_{>0}(H)$ that is integral on $L$ with $\operatorname{det}(L, F)=1$. By Table 2.5.4. $G$ is conjugate to $\operatorname{Aut}_{\mathbb{Q}(\sqrt{-d})}\left(L, \mathfrak{p}_{5}^{a} F\right)$ for some $a \in\{0,1\}$ and $d \in\{2,10\}$. These groups are:

\begin{tabular}{|c|c|c|}
\hline form & $\mathbb{Q}(\sqrt{-2})$ & $\mathbb{Q}(\sqrt{-10})$ \\
\hline \hline$F$ & $\sqrt{-2}\left[\left(2_{+}^{1+6} \cdot \mathrm{Alt}_{8}\right): 2\right]_{8}$ & $\sqrt{-10}\left[\sqrt{-2}\left[\mathrm{GL}_{2}(3)\right]_{2} \underset{\sqrt{-2}}{\stackrel{2_{+}}{\otimes}} \sqrt{5}, \infty\left[\mathrm{SL}_{2}(5)\right]_{1}\right]_{8}$ \\
\hline $\mathfrak{p}_{5} F$ & $\sqrt{\sqrt{-2}}\left[\mathrm{GL}_{2}(3)\right]_{2} \underset{\sqrt{-2}}{\otimes} \infty, 5\left[\mathrm{SL}_{2}(5): 2\right]$ & $\sqrt{-10}\left[\sqrt{-2}\left[\mathrm{GL}_{2}(3)\right]_{2} \underset{\sqrt{-2}}{\stackrel{2_{-}}{\otimes}, \infty}\left[\mathrm{SL}_{2}(5)\right]_{1}\right]_{8}$ \\
\hline
\end{tabular}

Note that this also handles the case where $[G: N]=4$.

- If $E(G) \simeq{ }_{\infty, 3}\left[\mathrm{SL}_{2}(9)\right]_{2}$, then $G$ contains a normal subgroup $N$ conjugate to ${ }_{\infty, 2}\left[\mathrm{SL}_{2}(3)\right]_{2} \otimes_{i} \infty_{, 3}\left[\mathrm{SL}_{2}(9)\right]_{2}$. Then $\operatorname{End}(\bar{N}) \simeq \mathcal{Q}_{2,3}$ and $\operatorname{Out}\left(\mathrm{SL}_{2}(9)\right) \simeq C_{2} \times C_{2}$ but one outer automorphism interchanges $\chi_{4 a}$ and $\chi_{4 b}$. Therefore $G / N \leq C_{2} \times C_{2}$. Remark 2.2.17 shows that $G$ contains a normal subgroup conjugate to $G_{1}:=$ $\infty, 2\left[\mathrm{SL}_{2}(3)\right]_{1} \underset{i}{\otimes_{\infty, 3}}\left[\mathrm{SL}_{2}(9)\right]_{2}$ or $G_{2}:=\infty_{\infty, 2}\left[\mathrm{SL}_{2}(3)\right]_{1} \underset{\sqrt{-3}}{\otimes}\left(\infty, 3\left[\mathrm{SL}_{2}(9)\right]_{2} \cdot 2\right)$. (Note that $\sqrt{2}, \infty\left[\tilde{S}_{4}\right]_{1} \otimes_{\infty, 3} \infty, 3\left[\mathrm{SL}_{2}(9)\right]_{2}$ is not symplectic and $\sqrt{-2}\left[\mathrm{GL}_{2}(3)\right]_{2} \otimes_{\infty, 3}\left[\mathrm{SL}_{2}(9)\right]_{2}$ is an irreducible subgroup of $\mathrm{GL}_{32}(\mathbb{Q})$ since $\mathbb{Q}(\sqrt{-2})$ does not split $\left.\mathcal{Q}_{\infty, 3}\right)$. The group $G_{1}$ is s.i.m.f. and $G_{2}$ is only contained in $\sqrt{-3}\left[\operatorname{Sp}_{4}(3) \circ C_{3}\right]_{\sqrt{-3}}^{\otimes} \infty\left[\operatorname{SL}_{2}(3)\right]_{1}$.

\section{Lemma 4.9.41}

(a) If $F(G) \simeq C_{4}$ then $G$ is conjugate to ${ }_{i}\left[C_{4}\right]_{1} \otimes E_{8},{ }_{i}\left[C_{4}\right]_{1} \otimes M_{8,3}, \infty, 3\left[\mathrm{SL}_{2}(7)\right]_{4} \circ C_{4}$, $\sqrt{-5}\left[\left(\left(\mathrm{SL}_{2}(5) \circ \mathrm{SL}_{2}(5)\right): 2\right) \stackrel{2-}{\bigotimes}_{i}\left[C_{4}\right]_{1}\right]_{8}$ or $\left.\sqrt{-5}\left[\left(\mathrm{SL}_{2}(5) \circ \mathrm{SL}_{2}(5)\right): 2\right) \stackrel{2_{+}}{\bigotimes_{i}}\left[C_{4}\right]_{1}\right]_{8}$.

(b) $F(G)$ is not conjugate to $D_{8}$.

Proof: Suppose $F(G) \simeq C_{4}$ or $D_{8}$. So $E(G)$ embeds into $\mathrm{GL}_{8}(\mathbb{Q}(i))$. By Table 2.5.1. $E(G)$ is isomorphic to $\mathrm{Alt}_{5}, \mathrm{SL}_{2}(5)$ (with character $4 \chi_{2 a b}$ ), $\mathrm{SL}_{2}(5) \circ$ $\mathrm{SL}_{2}(5), \mathrm{L}_{2}(7), \mathrm{SL}_{2}(7)$ (2 representations), $\mathrm{SL}_{2}(9), 2 . \mathrm{Alt}_{7}$ or $2 . O_{8}^{+}(2)$. (Note that $\left.\mathrm{O}_{3}\left(\mathcal{B}^{\circ}\left(\mathrm{Sp}_{4}(3)\right)\right) \simeq C_{3}\right)$.

Since $F(G)$ and $\operatorname{Out}(F(G)) \simeq C_{2}$ are 2-groups, so is $C:=C_{G}(E(G))$. Thus $F(G)=C$ and therefore $G / F^{*}(G) \leq \operatorname{Out}(E(G))$. 
- If $E(G) \simeq$ Alt $_{5}$, then $G=\mathcal{B}^{\circ}(N) \simeq \pm S_{5} \otimes F(G)$ is reducible.

- If $E(G) \simeq \sqrt{5, \infty}\left[\operatorname{SL}_{2}(5)\right]_{1}$ then $\left[G: F^{*}(G)\right] \leq 2$. Thus $F^{*}(G)={ }_{\sqrt{5}, \infty}\left[\operatorname{SL}_{2}(5)\right]_{1} \otimes D_{8}$ by Lemma 2.2.1. The commuting algebra of $F^{*}(G)$ is isomorphic to $\mathcal{Q}_{\sqrt{5}, \infty}$. The two groups ${ }_{\infty, 5}\left[\mathrm{SL}_{2}(5) \cdot 2\right]_{2} \otimes D_{8}$ and ${ }_{\infty, 5}\left[\mathrm{SL}_{2}(5): 2\right]_{2} \otimes D_{8}$ have $\mathcal{Q}_{\infty, 5}$ as commuting algebras. The two groups $\sqrt{5, \infty}\left[\mathrm{SL}_{2}(5)\right]_{1}{ }^{2_{+}} D_{8}$ and ${ }_{\sqrt{5}, \infty}\left[\mathrm{SL}_{2}(5)\right]_{1} \stackrel{2_{-}}{\otimes} D_{8}$ have $\mathcal{Q}_{\infty, 2}$ as commuting algebras. These four groups cannot be s.i.m.f. since their commuting algebras contain nontrivial torsion units.

- If $E(G) \simeq{ }_{\infty, 3}\left[\mathrm{SL}_{2}(9)\right]_{2}$ then $\left[G: F^{*}(G)\right] \leq 2$ since one outer automorphism of $\mathrm{SL}_{2}(9)$ interchanges $\chi_{4 a}$ and $\chi_{4 b}$. So $F^{*}(G) \simeq{ }_{\infty, 3}\left[\mathrm{SL}_{2}(9)\right]_{2} \otimes D_{8}$ by Lemma 2.2.1. This group has $\mathcal{Q}_{\infty, 3}$ as commuting algebra and is self-centralizing in $G$. By Remark 2.2.17 one finds that $G$ is a proper subgroup of $\sqrt{-3}\left[\operatorname{Sp}_{4}(3) \circ C_{3}\right]_{4}^{2}$.

- If $E(G) \simeq{ }_{\infty, 3}\left[\mathrm{SL}_{2}(7)\right]_{4}$, then $G \simeq{ }_{\infty, 3}\left[\mathrm{SL}_{2}(7)\right]_{4} \circ C_{4}$ by Lemma 4.9.3.

In all other cases, let $H \leq F(G)$ be the characteristic subgroup of order 4 and set $N:=H E(G)$.

- If $E(G) \simeq \mathrm{SL}_{2}(5) \circ \mathrm{SL}_{2}(5)$ then $B:=\mathcal{B}^{o}(N) \simeq\left(\left(\mathrm{SL}_{2}(5) \circ \mathrm{SL}_{2}(5)\right): 2\right) \otimes_{i}\left[C_{4}\right]_{1}$. Then $B$ has an irreducible cyclic subgroup of order 60 and $G / B \leq \operatorname{Out}(B)$ shows $\Pi(|G|)=\{2,3,5\}$. It follows from Theorem 4.9.8 that $G$ is conjugate to $\sqrt{-5}\left[\left(\left(\mathrm{SL}_{2}(5) \circ \mathrm{SL}_{2}(5)\right): 2\right) \stackrel{2+}{\bigotimes}_{i}\left[C_{4}\right]_{1}\right]_{8}$ or $\left.\sqrt{-5}\left[\left(\mathrm{SL}_{2}(5) \circ \mathrm{SL}_{2}(5)\right): 2\right) \stackrel{2_{-}}{\bigotimes_{i}}\left[C_{4}\right]_{1}\right]_{8}$.

- If $E(G) \simeq \mathrm{L}_{2}(7)$ then $\mathcal{B}^{o}(N) \simeq{ }_{i}\left[C_{4}\right]_{1} \otimes\left(\mathrm{L}_{2}(7) .2\right)={ }_{i}\left[C_{4}\right]_{1} \otimes M_{8,3}$ is already s.i.m.f.

- If $E(G) \simeq \mathrm{SL}_{2}(7)$ with character $2 \chi_{4 a b}$, then $N \simeq \mathrm{SL}_{2}(7) \otimes_{i}\left[C_{4}\right]_{1}$ has $C:=$ $\mathbb{Q}(i, \sqrt{7})$ as commuting algebra with totally real subfield $K \simeq \mathbb{Q}(\sqrt{7})$. Using part (a) of Remark 2.2.8 one finds a $N$-normal critical lattice $L$ such that $\operatorname{End}_{\mathbb{Z} N}(L)=\mathbb{Z}_{C}$ and some $F \in \mathcal{F}_{>0}(N)$ that is integral on $L$ with $\operatorname{det}(L, F)=1$. Since $\mathbb{Z}_{K,>0}^{*}=\operatorname{Nr}_{C / K}\left(\mathbb{Z}_{C}^{*}\right)$, we may restrict ourselves to one class of totally positive units. Further one finds some $x \in N_{\mathrm{GL}_{16}(\mathbb{Q})}(N) \cap \mathrm{GL}_{16}(L)$ such that $\mathfrak{p}_{3}^{x}=\mathfrak{p}_{3}^{\prime}$ (and $x$ necessarily fixes $\mathfrak{p}_{2}$ and $\mathfrak{p}_{7}$ ). Since $\Pi(|G|)=\{2,3,7\}$, it follows from Table 2.5.4 that $G$ is conjugate to $\operatorname{Aut}_{\mathbb{Q}(\sqrt{-d})}\left(L, \mathfrak{p}_{2}^{a} \mathfrak{p}_{3}^{b} \mathfrak{p}_{7}^{b} F\right)$ for some $a, b \in\{0,1\}$ and $d \in\{1,7\}$. If $b=1$ these groups equal $N$. The other four groups are:

\begin{tabular}{|c|c|c|}
\hline form & $\mathbb{Q}(i)$ & $\mathbb{Q}(\sqrt{-7})$ \\
\hline \hline$F$ & $\succ_{i}\left[C_{4}\right]_{1}^{8}$ & $\lessgtr \sqrt{-7}^{2}\left[2 . \mathrm{Alt}_{7}\right]_{8}^{2}$ \\
\hline $\mathfrak{p}_{2} F$ & ${ }_{i}\left[\left(2_{+}^{1+6} \otimes C_{4}\right) \cdot \mathrm{Sp}_{6}(2)\right]_{8}$ & $\left.\subsetneq \sqrt{-7}^{1+\mathrm{Alt}_{7}}\right]_{8}^{2}$ \\
\hline
\end{tabular}

- If $E(G) \simeq 2$. Alt $_{7}$ then $N \simeq \sqrt{-7}\left[2 . \mathrm{Alt}_{7}\right]_{4} \otimes_{i}\left[C_{4}\right]_{1}$ has $C:=\mathbb{Q}(i, \sqrt{7})$ as commuting algebra. The group $N$ fixes only one lattice $L$ and there exists some $F \in \mathcal{F}_{>0}(N)$ that is integral on $L$ with $\operatorname{det}(L, F)=1$. As before, there are eight candidates to check:

\begin{tabular}{|c|c|c|}
\hline form & $\mathbb{Q}(i)$ & $\mathbb{Q}(\sqrt{-7})$ \\
\hline \hline$F$ & ${ }_{i}\left[C_{4}\right]_{1} \otimes E_{8}$ & $\sqrt{-7}\left[2 . \mathrm{Alt}_{7}\right]_{8}^{2}$ \\
\hline $\mathfrak{p}_{2} F$ & ${ }_{i}\left[\left(2_{+}^{1+6} \otimes C_{4}\right) \cdot \mathrm{Sp}_{6}(2)\right]_{8}$ & $\underset{\sqrt{-7}}{\leq}\left[2 . \mathrm{Alt}_{7}\right]_{8}^{2}$ \\
\hline $\mathfrak{p}_{3} \mathfrak{p}_{7} F$ & $N$ & $\tilde{V}_{-7}\left[2 . \mathrm{Alt}_{7}\right]_{8} \otimes_{\sqrt{-7}} \infty, 3$ \\
& & $N$ \\
\hline $\mathfrak{p}_{2} \mathfrak{p}_{3} \mathfrak{p}_{7} F$ & $N$ & $N]_{1}$ \\
\hline
\end{tabular}


- If $E(G) \simeq 2 . O_{8}^{+}(2)$ then $\mathcal{B}^{o}(N) \simeq{ }_{i}\left[C_{4}\right]_{1} \otimes E_{8}$ is already s.i.m.f..

Lemma 4.9.42 $F(G)$ is not conjugate to $C_{2}$.

Proof: Otherwise $G / \pm E(G) \leq \operatorname{Out}(E(G))$. Table 2.5.1 shows that $E(G)$ is isomorphic to $\mathrm{Alt}_{5}, \mathrm{SL}_{2}(5)$ (with character $\left.4 \chi_{2 a b}\right), \mathrm{SL}_{2}(5) \circ \mathrm{SL}_{2}(5), \mathrm{SL}_{2}(7), \mathrm{L}_{2}(7)$ (2 representations), $\mathrm{SL}_{2}(9), 2 . \mathrm{Alt}_{7}$ or $2 . \mathrm{O}_{8}^{+}(2)$.

The case that $E(G)$ is conjugate to $\mathrm{SL}_{2}(7)$ with character $2 \chi_{8}$ has been handled in Lemma 4.9.3. If $E(G) \simeq \mathrm{L}_{2}(7)$ then $\mathcal{B}^{\circ}(E(G)) \simeq \pm \mathrm{L}_{2}(7)$.2. Since Out $(E(G)) \simeq C_{2}$, we get $G= \pm \mathrm{L}_{2}(7) .2$ is reducible. Similarly, if $E(G) \simeq 2 . O_{8}^{+}(2)$ then $\mathcal{B}^{\circ}(E(G)) \simeq E_{8}$. Since $\operatorname{Out}(E(G)) \simeq C_{2}$, we get $G=E_{8}$ is reducible.

Suppose $E(G) \simeq \mathrm{SL}_{2}(5) \circ \mathrm{SL}_{2}(5)$. Then $G$ contains a normal subgroup $B:=$ $\mathcal{B}^{\circ}(E(G)) \simeq\left(\left(\mathrm{SL}_{2}(5) \circ \mathrm{SL}_{2}(5)\right): 2\right)$ and $G / B \leq \operatorname{Out}(B) \simeq C_{2} \times C_{2}$. Only one class of outer automorphisms can be realized in $\mathrm{GL}_{16}(\mathbb{Q})$ and both extensions (split and non-split) are reducible. Thus this case cannot happen.

In all other cases $E(G)$ is reducible, has a commuting algebra which is not isomorphic to $K^{2 \times 2}$ for some totally real number field $K$ and $G / \pm E(G) \leq C_{2}$. (Note that Out $\left(\mathrm{SL}_{2}(9)\right) \simeq C_{2} \times C_{2}$ but one outer automorphism interchanges $\chi_{4 a}$ and $\chi_{4 b}$.) This contradicts Lemma 2.2.1. 


\subsection{Dimension 18}

Theorem 4.10.1 The s.i.m.f. subgroups $G$ of $\operatorname{Sp}_{18}(\mathbb{Q})$ are

\begin{tabular}{|c|c|c|c|c|c|}
\hline \# & G & $|G|$ & $\mid \mathcal{Z}(G)$ & $L_{\min }$ & $\begin{array}{c}\text { r.i.m.f. } \\
\text { supergroups }\end{array}$ \\
\hline$\overline{[2,1,9]}$ & ${ }_{i}\left[C_{4}\right]_{1}^{9}$ & $22^{25} \cdot 3^{4} \cdot 5 \cdot 7$ & 3 & $\overline{[1,1,36]}$ & $\overline{B_{18}}$ \\
\hline 1 & ${ }_{i}\left[C_{4}\right]_{1} \otimes A_{9}$ & $2^{10} \cdot 3^{4} \cdot 5^{2} \cdot 7$ & 12 & {$[1,2,180]$} & $A_{9}^{2}$ \\
\hline$\overline{[2,2,9]}$ & $\overline{\overline{\sqrt{-3}}\left[C_{6}\right]_{1}^{9}}$ & $2^{16} \cdot 3^{13} \cdot 5 \cdot 7$ & 1 & $\overline{\left[3^{9}, 2,54\right]}$ & 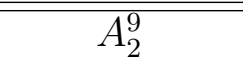 \\
\hline$[6,1,3]$ & $\sqrt{-3}\left[ \pm 3_{+}^{1+2}: \mathrm{SL}_{2}(3)\right]_{3}^{3}$ & $2^{13} \cdot 3^{13}$ & 2 & {$\left[3^{3}, 2,216\right]$} & $E_{6}^{3}$ \\
\hline 2 & $\sqrt{-3}\left[ \pm 3_{+}^{1+4}: \operatorname{Sp}_{4}(3)\right]_{9}$ & $2^{8} \cdot 3^{9} \cdot 5$ & 2 & {$\left[3^{5}, 4,6480\right]$} & $H_{1}$ \\
\hline 3 & $\sqrt{-3}\left[C_{6}\right]_{1} \otimes A_{9}$ & $2^{9} \cdot 3^{5} \cdot 5^{2} \cdot 7$ & 4 & {$\left[2^{2} \cdot 3^{9} \cdot 5^{2}, 4,270\right]$} & $A_{2} \otimes A_{9}$ \\
\hline 4 & $\sqrt{-3}\left[ \pm 3 . M_{10}\right]_{9}$ & $2^{5} \cdot 3^{3} \cdot 5$ & 2 & {$\left[3^{9} \cdot 5^{6}, 6,180\right]$} & {$\left[ \pm 3 . \mathrm{Alt}_{6} \cdot 2^{2}\right]_{18}$} \\
\hline$[6,2,3]$ & $\sqrt{-7}\left[ \pm \mathrm{L}_{2}(7)\right]_{3}^{3}$ & $2^{13} \cdot 3^{4} \cdot 7^{3}$ & 1 & {$\left[7^{9}, 4,126\right]$} & $\left(A_{6}^{(2)}\right)^{3}$ \\
\hline 5 & $\sqrt{-7}\left[ \pm\left(\mathrm{L}_{2}(7) \underset{\sqrt{-7}}{\otimes} \mathrm{L}_{2}(7)\right): 2\right]_{9}$ & $2^{8} \cdot 3^{2} \cdot 7^{2}$ & 1 & {$\left[7^{9}, 6,336\right]$} & $\mathrm{H}_{2}$ \\
\hline 6 & $\sqrt{-19}\left[ \pm \mathrm{L}_{2}(19)\right]_{9}$ & $2^{3} \cdot 3^{2} \cdot 5 \cdot 19$ & 1 & {$\left[19^{9}, 10,342\right]$} & $\overline{A_{18}^{(5)}}$ \\
\hline
\end{tabular}

where $H_{1}:=\left[ \pm 3_{+}^{1+4}: \mathrm{Sp}_{4}(3) .2\right]_{18}$ and $H_{2}:=\left[ \pm\left(\mathrm{L}_{2}(7) \underset{\sqrt{-7}}{\stackrel{2}{\otimes}} \mathrm{L}_{2}(7)\right): 2\right]_{18}$ are r.i.m.f. subgroups of $\mathrm{GL}_{18}(\mathbb{Q})$.

Again, before we prove the theorem, we discuss the case that $G$ contains an irreducible cyclic subgroup $U$. If $| \pm U|=38$, then Theorem 3.1.1 shows that $G \simeq \sqrt{-19}\left[ \pm \mathrm{L}_{2}(19)\right]_{9}$. The remaining case $| \pm U|=54$ is handled below.

Lemma 4.10.2 If $G$ contains an irreducible cyclic subgroup $U$ of order 54 and $\Pi(|G|) \subseteq\{2,3,5,7,11,13\}$, then $G$ is conjugate to $\sqrt{-3}\left[C_{6}\right]_{1}^{9}$ or $\sqrt{-3}\left[ \pm 3_{+}^{1+2}: \mathrm{SL}_{2}(3)\right]_{3}^{3}$.

Proof: The centralizing algebra $C$ of $U$ is isomorphic to $\mathbb{Q}\left(\zeta_{54}\right)$ and has class number 1. Thus $G$ fixes only one lattice $L$. There exists some $F \in \mathcal{F}_{>0}(U)$ that is integral on $L$ with $\operatorname{det}(L, F)=3$. By Table 2.5.3, $G$ must be conjugate to $\operatorname{Aut}_{\mathbb{Q}(\sqrt{-3})}\left(L, \mathfrak{p}_{3}^{k} F\right)$ for some $0 \leq k \leq 4$. So the result is easily verified.

Proof (Theorem 4.10.1): Explicit calculations show that the table is correct and it yields s.i.m.f. matrix groups. The symplectic imprimitive matrix groups come from the s.p.i.m.f. subgroups of $\operatorname{Sp}_{2}(\mathbb{Q})$ and $\operatorname{Sp}_{6}(\mathbb{Q})$. It follows from Lemma 2.1.21 that ${ }_{\sqrt{-3}}\left[C_{6}\right]_{1}^{9}, \sqrt{-7}\left[ \pm \mathrm{L}_{2}(7)\right]_{3}^{3}$ and $\sqrt{-3}\left[ \pm 3_{+}^{1+2}: \mathrm{SL}_{2}(3)\right]_{3}^{3}$ are maximal finite. The group ${ }_{i}\left[C_{4}\right]_{1}^{9}$ fixes 3 lattices and has $\mathbb{Q}(i)$ as commuting algebra. One verifies that this group is also maximal finite.

It remains to classify the s.p.i.m.f. subgroups $G<\operatorname{Sp}_{18}(\mathbb{Q})$. This is done in the following three lemmas by discussing the various Fitting subgroups. In any case we have $F(G)=\prod_{p \in\{2,3,7\}} O_{p}(G)$ according to Corollary 2.1.16 and Theorem 3.1.1.

For the remainder of the section suppose $G<\operatorname{Sp}_{18}(\mathbb{Q})$ is s.p.i.m.f.. 
Lemma 4.10.3 $O_{7}(G)=1$.

Proof: By Table 2.5.2 the Fitting subgroup $F(G)$ must be conjugate to $\pm C_{7}$. Suppose first $E(G)=1$. By Lemma 2.2.1 $G$ must contain a normal subgroup $N \simeq \pm C_{7}: C_{3}$. But there exists only one such extension and this embeds into $\mathrm{GL}_{6}(\mathbb{Q})$. Since $[G: N] \leq 2$ this contradicts loc. cit.. So $E(G) \neq 1$ embeds into $\mathrm{GL}_{3}\left(\mathbb{Q}\left(\zeta_{7}\right)\right)$. Thus $E(G) \simeq \mathrm{L}_{2}(7)$ by Table 2.5.1. Then $F^{*}(G) \simeq \pm C_{7} \underset{\sqrt{-7}}{\otimes} \mathrm{L}_{2}(7)$ is irreducible and fixes only one lattice L. Let $C \simeq \mathbb{Q}\left(\zeta_{14}\right)$ denote the commuting algebra of $F^{*}(G)$ and let $K \simeq \mathbb{Q}\left(\theta_{14}\right)$ be its maximal real subfield. Since $G / F^{*}(G) \leq C_{6} \times C_{2}$, we only have to consider the prime ideals over 2,3 and 7. One finds some $F \in \mathcal{F}_{>0}\left(F^{*}(G)\right)$ that is integral on $L$ such that $\operatorname{det}(L, F)=7^{3}$. Further $C$ has only one totally complex subfield which is $\mathbb{Q}(\sqrt{-7})$. Hence it follows from Table 2.5.3, that $G$ is conjugate to $\operatorname{Aut}_{\mathbb{Q}(\sqrt{-7})}\left(L, \mathfrak{p}_{7}^{a} F\right)$ with $a \in\{0,1\}$ (for $a \geq 2$ the lattice is not normalized). Both automorphism groups are contained in $\sqrt{-7}\left[ \pm\left(\mathrm{L}_{2}(7) \underset{\sqrt{-7}}{\otimes} \mathrm{L}_{2}(7)\right): 2\right]_{9}$.

Lemma 4.10.4 If $O_{3}(G) \neq 1$ then either

(a) $O_{3}(G) \simeq 3_{+}^{1+4}$ and $G$ is conjugate to $\sqrt{-3}\left[ \pm 3_{+}^{1+4}: \mathrm{Sp}_{4}(3)\right]_{9}$.

(b) $O_{3}(G) \simeq C_{3}$ and $G$ is conjugate to $\sqrt{-3}\left[C_{6}\right]_{1} \otimes A_{9}$ or $\sqrt{-3}\left[ \pm 3 . M_{10}\right]_{9}$.

Proof: If $O_{3}(G)$ is irreducible, it is conjugate to $3_{+}^{1+4}$ or $3_{+}^{1+2} \underset{\sqrt{-3}}{\otimes} C_{9}$ since $O_{3}(G) \not C_{27}$ by Lemma 4.10.2. If $O_{3}(G)$ embeds irreducibly into $\mathrm{GL}_{6}(\mathbb{Q})$, it is conjugate to $C_{9}$ or $3_{+}^{1+2}$. Otherwise $O_{3}(G) \simeq C_{3}$ embeds irreducibly into $\mathrm{GL}_{2}(\mathbb{Q})$.

- If $O_{3}(G) \simeq 3_{+}^{1+4}$ then $\mathcal{B}^{o}\left(O_{3}(G)\right) \simeq \sqrt{-3}\left[ \pm 3_{+}^{1+4}: \operatorname{Sp}_{4}(3)\right]_{9}$ is already s.i.m.f..

- If $O_{3}(G) \simeq 3_{+}^{1+2} \underset{\sqrt{-3}}{\otimes} C_{9}$ then $G$ contains a normal subgroup $N$ conjugate to $\pm\left(3_{+}^{1+2} \underset{\sqrt{-3}}{\otimes} C_{9}\right): \mathrm{SL}_{2}(3)$. The commuting algebra of $N$ is isomorphic to $\mathbb{Q}\left(\zeta_{9}\right)$. Thus $G / N \leq C_{6}$ and therefore $\Pi(|G|)=\{2,3\}$. There exists a normal critical set of two lattices $\left\{L_{1}, L_{2}\right\}$ and $F \in \mathcal{F}_{>0}(N)$ that is integral on the $L_{i}$ with $\operatorname{det}\left(L_{i}, F\right) \in\{3,27\}$. By Table 2.5.3 we only have to consider the groups $\operatorname{Aut}_{\mathbb{Q}(\sqrt{-3})}\left(L_{i}, \mathfrak{p}_{3}^{a} F\right)$ with $a \in\{0,1\}$. These groups are subgroups of the s.i.m.f. groups $\sqrt{-3}\left[ \pm 3_{+}^{1+4}: \mathrm{Sp}_{4}(3)\right]_{9}$ or $\sqrt{-3}\left[ \pm 3_{+}^{1+2}: \mathrm{SL}_{2}(3)\right]_{3}^{3}$.

- If $O_{3}(G) \simeq C_{9}$ or $3_{+}^{1+2}$ then $C_{G}\left(O_{3}(G)\right)$ embeds into $\mathrm{GL}_{3}\left(\mathbb{Q}\left(\zeta_{k}\right)\right)$ with $k \in\{3,9\}$. In particular $F^{*}(G)= \pm O_{3}(G)$. Let $N:=\mathcal{B}^{\circ}\left(O_{3}(G)\right) \unlhd G$. Then $N$ is conjugate to $\pm C_{9}$ or $\pm 3_{+}^{1+2}: \mathrm{SL}_{2}(3)$. In the first case, let $P \in \operatorname{Syl}_{3}(G)$. Then $[G: \pm P] \leq 2$ shows $P=N$ and therefore $[G: N] \leq 2$. In the second case, it follows from $\operatorname{Out}\left(O_{3}(G)\right) \simeq \mathrm{GL}_{2}(3)$ that $[G: N] \leq 2$. So both cases contradict Lemma 2.2.1.

- If $O_{3}(G) \simeq C_{3}$, then $C_{G}\left(O_{3}(G)\right)$ embeds into $\mathrm{GL}_{9}(\mathbb{Q}(\sqrt{-3}))$. So $O_{2}(G)= \pm I_{18}$ and it follows from Table 2.5.1 that $E(G)$ is conjugate to $\mathrm{Alt}_{10}$ or $3 . \mathrm{Alt}_{6}$. In any case $\mathcal{B}^{\circ}\left(F^{*}(G)\right)$ is already s.i.m.f. and conjugate to one of the groups stated above. 
Lemma 4.10.5 If $F(G)=O_{2}(G)$, then $G$ is conjugate to ${ }_{i}\left[C_{4}\right]_{1} \otimes A_{9}, \sqrt{-19}\left[ \pm \mathrm{L}_{2}(19)\right]_{9}$ or $\sqrt{-7}\left[ \pm\left(\mathrm{L}_{2}(7) \underset{\sqrt{-7}}{\otimes} \mathrm{L}_{2}(7)\right): 2\right]_{9}$.

Proof: Suppose first $F(G) \neq \pm I_{18}$. Then $F(G)$ is isomorphic to $C_{4}$ or $D_{8}$. Thus $G$ contains a normal subgroup $N \simeq C_{4}$ and $E(G)$ embeds into $\mathrm{GL}_{9}(\mathbb{Q}(i))$. By Table 2.5.1 $E(G)$ is conjugate to $\mathrm{Alt}_{10}$. So $G$ contains an irreducible normal subgroup $B:=$ $N \mathcal{B}^{\circ}(E(G)) \simeq_{i}\left[C_{4}\right]_{1} \otimes A_{9}$. The group $B$ fixes 12 lattices and has $\mathbb{Q}(i)$ as commuting algebra. One easily checks that it is s.i.m.f..

If $F(G)= \pm I_{18}$, then $E(G) \neq 1$ embeds irreducibly into $\mathrm{GL}_{k}(\mathbb{Q})$ for $k \in\{6,9,18\}$ by Table 2.5.1.

(a) If $k=6$ then $E(G) \simeq \operatorname{Alt}_{5}, \operatorname{Alt}_{7}, \mathrm{~L}_{2}(7)$ or $U_{4}(2)$. In any case $\left[G: \mathcal{B}^{o}(E(G))\right] \leq 2$ contradicts Lemma 2.2.1.

(b) If $k=9$ then $E(G) \simeq \operatorname{Alt}_{10}$ and $\mathcal{B}^{o}(G) \simeq A_{9}$ implies $G=\mathcal{B}^{\circ}(G)$ is reducible.

(c) If $k=18$ then $E(G) \simeq \mathrm{L}_{2}(19)$ or $\mathrm{L}_{2}(7) \underset{\sqrt{-7}}{\otimes \mathrm{L}_{2}}(7)$ since $O_{3}\left(\mathcal{B}^{o}\left(3 . \mathrm{Alt}_{6}\right)\right)=C_{3}$ and $\mathrm{Alt}_{5} \otimes \mathrm{Alt}_{5}$ fixes no skewsymmetric form. The group $\sqrt{-19}\left[ \pm \mathrm{L}_{2}(19)\right]_{9}$ is s.i.m.f. by Theorem 3.2.1. In the second case $\mathcal{B}^{\circ}(E(G)) \simeq \underset{\sqrt{-7}}{ }\left[ \pm\left(\mathrm{L}_{2}(7) \underset{\sqrt{-7}}{\otimes} \mathrm{L}_{2}(7)\right): 2\right]_{9}$ is irreducible and fixes only one lattice. One checks that it is s.i.m.f.. 


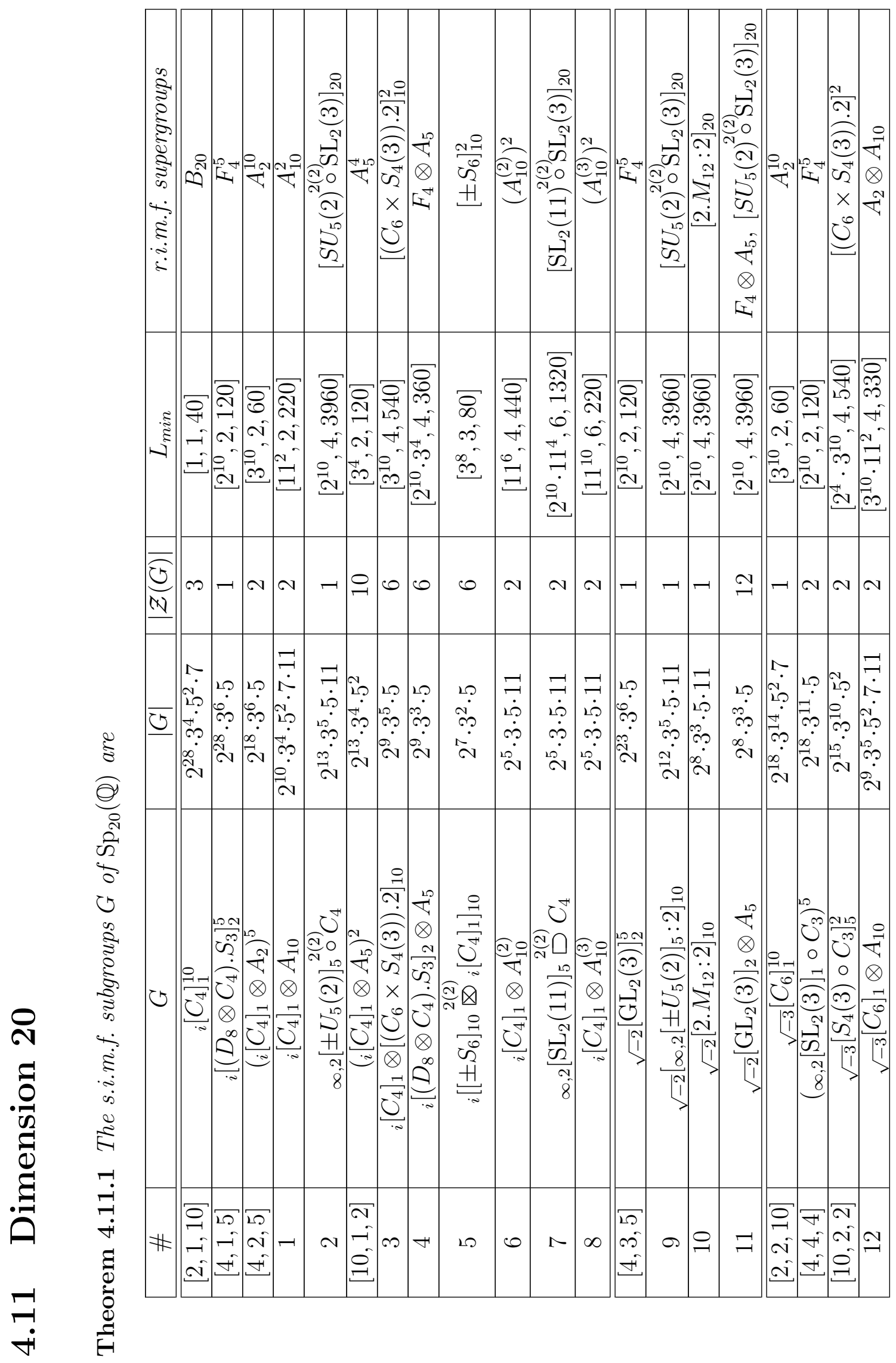




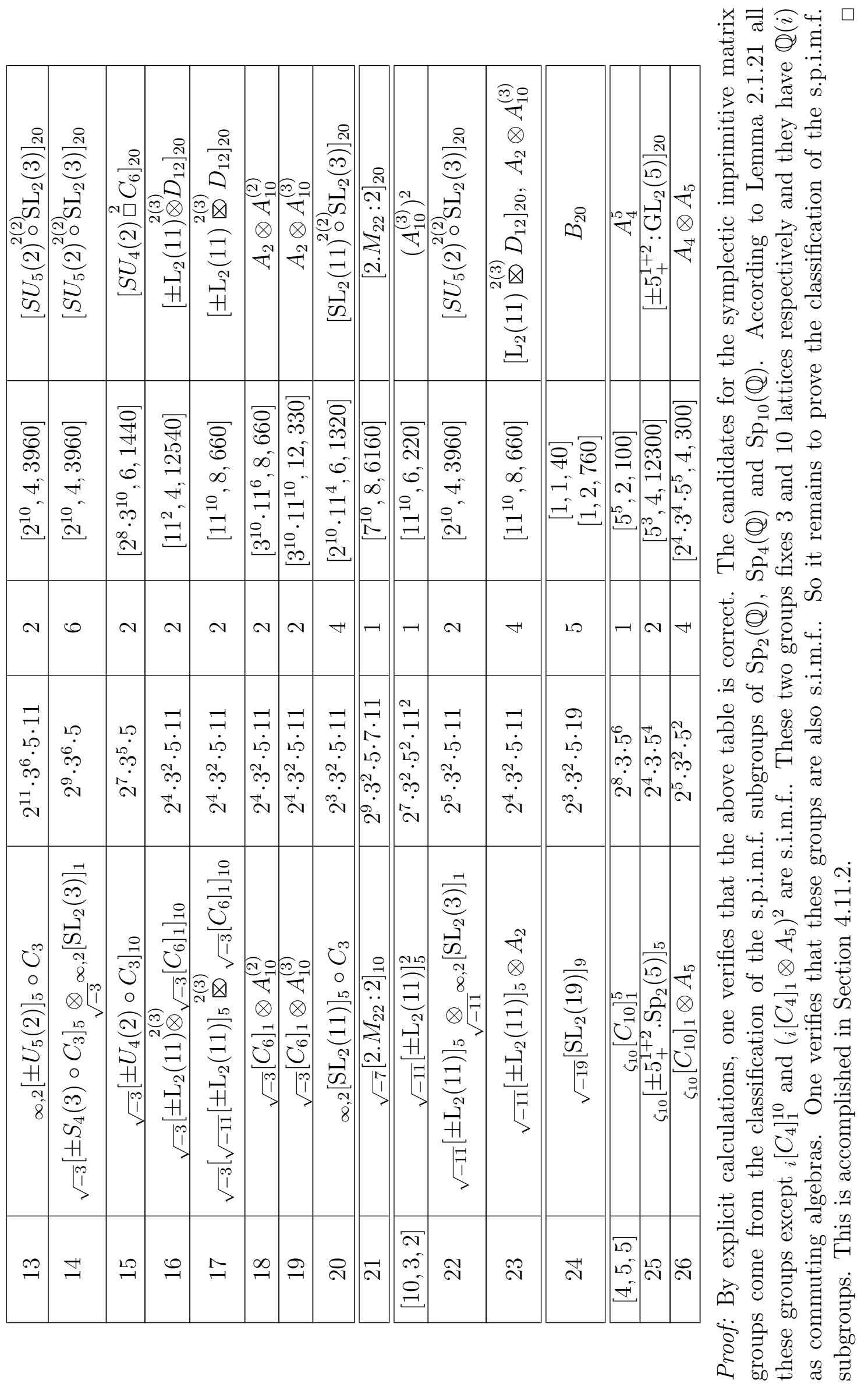




\subsubsection{Irreducible cyclic subgroups}

If a s.i.m.f. matrix group $G<\operatorname{Sp}_{20}(\mathbb{Q})$ contains an irreducible cyclic subgroup $U$, then $| \pm U| \in\{50,44,66\}$. These cases are handled below.

Lemma 4.11.2 Suppose $G$ contains an irreducible cyclic subgroup $U$ of order 50 and $\Pi(|G|) \subseteq\{2,3,5\}$ or there exists some $(L, F) \in \mathcal{Z}(G) \times \mathcal{F}_{>0}(G)$ such that $F$ is integral on $L$ with $\operatorname{det}(L, F) \subseteq\{2,3,5\}$. Then $G$ is conjugate to $\zeta_{10}\left[C_{10}\right]_{1}^{5}$.

Proof: The commuting algebra of $U$ is isomorphic to $\mathbb{Q}\left(\zeta_{50}\right)$ and has class number 1 . Thus $G$ fixes only one lattice $L$. Further there exists some $F \in \mathcal{F}_{>0}(U)$ that is integral on $L$ with $\operatorname{det}(L, F)=5$. By Table 2.5.3, $G$ must be conjugate to $\operatorname{Aut}_{\mathbb{Q}\left(\zeta_{10}\right)}\left(L, \mathfrak{p}_{5}^{a} F\right)$ for some $0 \leq a \leq 4$. All these groups are subgroups of $\zeta_{10}\left[C_{10}\right]_{1}^{5}$.

Lemma 4.11.3 Suppose $G$ contains an irreducible cyclic subgroup $U$ of order 44 and $\Pi(|G|) \subseteq\{2,3,5,11\}$ or there exists some $(L, F) \in \mathcal{Z}(G) \times \mathcal{F}_{>0}(G)$ such that $F$ is integral on $L$ with $\operatorname{det}(L, F) \subseteq\{2,3,5,11\}$. Then $G$ is conjugate to one of

$$
\begin{gathered}
\sqrt{-11}\left[ \pm \mathrm{L}_{2}(11)\right]_{5}^{2}, \sqrt{-11}\left[ \pm \mathrm{L}_{2}(11)\right]_{5} \underset{\sqrt{-11}}{\otimes} \infty\left[\mathrm{SL}_{2}(3)\right]_{1}, \infty, 2\left[ \pm U_{5}(2)\right]_{5} \stackrel{2(2)}{\circ} C_{4}, \\
\infty, 2\left[ \pm \mathrm{SL}_{2}(11)\right]_{5} \stackrel{2(2)}{\square} C_{4} \text { or }{ }_{i}\left[C_{4}\right]_{1} \otimes H \text { with } H \in\left\{A_{10}, A_{10}^{(2)}, A_{10}^{(3)}\right\} .
\end{gathered}
$$

Proof: The commuting algebra $C:=\operatorname{End}(\bar{U}) \simeq \mathbb{Q}\left(\zeta_{44}\right)$ and has class number 1 . Thus $G$ fixes only one lattice $L$. Further there exists some $F \in \mathcal{F}_{>0}(U)$ that is integral on $L$ such that $\operatorname{det}(L, F)=11^{2}$. Let $K \simeq \mathbb{Q}\left(\theta_{44}\right)$ be the maximal totally real subfield of $C$. There exists some $\sigma \in \operatorname{Gal}(C / K)$ that interchanges $\mathfrak{p}_{5}$ and $\mathfrak{p}_{5}^{\prime}$. Further $\sigma$ is conjugation by some $x \in N_{\mathrm{GL}_{16}(\mathbb{Q})}(U) \cap \mathrm{GL}(L)$ with $x F x^{\text {tr }}=F$. So Table 2.5.3 shows that $G$ is conjugate to $\operatorname{Aut}_{\mathbb{Q}(\sqrt{-d})}\left(L, \mathfrak{p}_{2}^{a} \mathfrak{p}_{5}^{b} \mathfrak{p}_{11}^{2 c-a} F\right)$ for some $a, b \in\{0,1\}, 0 \leq c \leq 2$ and $d \in\{1,11\}$.

One finds $\operatorname{Aut}_{\mathbb{Q}(\sqrt{-11})}\left(L, \mathfrak{p}_{2}^{a} \mathfrak{p}_{5}^{b} \mathfrak{p}_{11}^{2 c-a} F\right) \leq\left\{\begin{array}{ll}\sqrt{-11}\left[ \pm \mathrm{L}_{2}(11)\right]_{5}^{2} & \text { if } a=0 \\ \sqrt{-11}\left[ \pm \mathrm{L}_{2}(11)\right]_{5} \otimes_{\sqrt{-11}} \infty, 2\left[\mathrm{SL}_{2}(3)\right]_{1} & \text { if } a=1\end{array}\right.$.

Further $\operatorname{Aut}_{\mathbb{Q}(\sqrt{-11})}\left(L, \mathfrak{p}_{2}^{a} \mathfrak{p}_{5} \mathfrak{p}_{11}^{2 c-a} F\right)=\operatorname{Aut}_{\mathbb{Q}(i)}\left(L, \mathfrak{p}_{2}^{a} \mathfrak{p}_{5} \mathfrak{p}_{11}^{2 c-a} F\right)$. The remaining 6 automorphism groups are given below.

\begin{tabular}{|c|c|c|c|}
\hline form & $\mathbb{Q}(i)$ & form & $\mathbb{Q}(i)$ \\
\hline \hline$F$ & ${ }_{i}\left[C_{1}\right]_{1} \otimes A_{10}$ & $\mathfrak{p}_{2} \mathfrak{p}_{11}^{-1} F$ & $\infty, 2\left[ \pm U_{5}(2)\right]_{5}^{2(2)}{ }^{\circ} C_{4}$ \\
\hline $\mathfrak{p}_{11}^{2} F$ & ${ }_{i}\left[C_{1}\right]_{1} \otimes A_{10}^{(2)}$ & $\mathfrak{p}_{2} \mathfrak{p}_{11} F$ & $\infty, 2\left[\mathrm{SL}_{2}(11)\right]_{5}{ }^{2(2)} C_{4}$ \\
\hline $\mathfrak{p}_{11}^{4} F$ & ${ }_{i}\left[C_{1}\right]_{1} \otimes A_{10}^{(3)}$ & $\mathfrak{p}_{2} \mathfrak{p}_{11}^{3} F$ & $\leq \infty, 2\left[\mathrm{SL}_{2}(11)\right]_{5}{ }^{2(2)} C_{4}$ \\
\hline
\end{tabular}


Lemma 4.11.4 Suppose $G$ contains an irreducible cyclic subgroup $U$ of order 66 and $\Pi(|G|) \subseteq\{2,3,5,7,11\}$ or there exists some $(L, F) \in \mathcal{Z}(G) \times \mathcal{F}_{>0}(G)$ such that $F$ is integral on $L$ with $\operatorname{det}(L, F) \subseteq\{2,3,5,7,11\}$. Then $G$ is conjugate to one of

$$
\begin{gathered}
\sqrt{-11}\left[ \pm \mathrm{L}_{2}(11)\right]_{5} \otimes A_{2}, \sqrt{-11}\left[ \pm \mathrm{L}_{2}(11)\right]_{5} \underset{\sqrt{-11}}{\otimes} \infty_{2}\left[\mathrm{SL}_{2}(3)\right]_{1}, \infty, 2\left[ \pm U_{5}(2)\right]_{5} \circ C_{3} \\
\infty, 2\left[\mathrm{SL}_{2}(11)\right]_{5} \circ C_{3}, \sqrt{-3}\left[ \pm \mathrm{L}_{2}(11)^{2(3)} \otimes_{\sqrt{-3}}\left[C_{6}\right]_{1}\right]_{10}, \sqrt{-3}\left[\sqrt{-11}\left[ \pm \mathrm{L}_{2}(11)\right]_{5}^{2(3)} \underset{\sqrt{-3}}{\otimes}\left[C_{6}\right]_{1}\right]_{10} \\
\text { or } \sqrt{-3}\left[C_{6}\right]_{1} \otimes H \text { with } H \in\left\{A_{10}, A_{10}^{(2)}, A_{10}^{(3)}\right\} .
\end{gathered}
$$

Proof: The commuting algebra $C$ of $U$ is isomorphic to $\mathbb{Q}\left(\zeta_{66}\right)$ and has class number 1 . Thus $G$ fixes only one lattice $L$. Further there exists some $F \in \mathcal{F}_{>0}(U)$ that is integral on $L$ such that $\operatorname{det}(L, F)=11^{2}$. Let $K \simeq \mathbb{Q}\left(\theta_{66}\right)$ be the maximal totally real subfield of $C$. There exists some $\sigma \in \operatorname{Gal}(C / K)$ that interchanges $\mathfrak{p}_{2}$ and $\mathfrak{p}_{2}^{\prime}$. Further $\sigma$ is conjugation by some $x \in N_{\mathrm{GL}_{20}(\mathbb{Q})}(U) \cap \mathrm{GL}(L)$ with $x F x^{\mathrm{tr}}=F$. So Table 2.5.3 shows that $G$ is conjugate to $\operatorname{Aut}_{\mathbb{Q}(\sqrt{-d})}\left(L, \mathfrak{p}_{2}^{a} \mathfrak{p}_{3}^{b} \mathfrak{p}_{11}^{2 c-a} F\right)$ for some $a, b \in\{0,1\}, 0 \leq c \leq 2$ and $d \in\{3,11\}$.

One checks that $\operatorname{Aut}_{\mathbb{Q}(\sqrt{-11})}\left(L, \mathfrak{p}_{2}^{a} \mathfrak{p}_{3}^{b} \mathfrak{p}_{11}^{2 c-a} F\right) \leq\left\{\begin{array}{ll}\sqrt{-11}\left[ \pm \mathrm{L}_{2}(11)\right]_{5} \otimes A_{2} & \text { if } a=0 \\ \sqrt{-11}\left[ \pm \mathrm{L}_{2}(11)\right]_{5} \otimes \mathrm{SL}_{2}(3) & \text { if } a=1\end{array}\right.$.

\begin{tabular}{|c|c|c|c|}
\hline form & $\mathbb{Q}(\sqrt{-3})$ & form & $\mathbb{Q}(\sqrt{-3})$ \\
\hline$F$ & $\sqrt{-3}\left[ \pm \mathrm{L}_{2}(11) \otimes^{2(3)} \otimes_{\sqrt{-3}}\left[C_{6}\right]_{1}\right]_{10}$ & $\mathfrak{p}_{3} F$ & $\sqrt{-3}\left[C_{6}\right]_{1} \otimes A_{10}$ \\
\hline $\mathfrak{p}_{11}^{2} F$ & $\lesseqgtr \sqrt{-3}\left[\sqrt{-11}\left[ \pm \mathrm{L}_{2}(11)\right]_{5} \stackrel{2(3)}{\otimes} \sqrt{-3}\left[C_{6}\right]_{1}\right]_{10}$ & $\mathfrak{p}_{3} \mathfrak{p}_{11}^{2} F$ & $\sqrt{-3}\left[C_{6}\right]_{1} \otimes A_{10}^{(2)}$ \\
\hline $\mathfrak{p}_{11}^{4} F$ & $\sqrt{-3}\left[\sqrt{-11}\left[ \pm \mathrm{L}_{2}(11)\right]_{5} \stackrel{2(3)}{\otimes} \sqrt{-3}\left[C_{6}\right]_{1}\right]_{10}$ & $\mathfrak{p}_{3} \mathfrak{p}_{11}^{4} F$ & $\sqrt{-3}\left[C_{6}\right]_{1} \otimes A_{10}^{(3)}$ \\
\hline $\begin{array}{l}\mathfrak{p}_{2} \mathfrak{p}_{11}^{-1} F \\
\mathfrak{p}_{2} \mathfrak{p}_{11}^{3} F\end{array}$ & $\begin{array}{c}\infty, 2\left[ \pm U_{5}(2)\right]_{5} \circ C_{3} \\
\lessgtr \sqrt{-11}\left[ \pm \mathrm{L}_{2}(11)\right]_{5} \underset{\sqrt{-11}}{\otimes} \infty, 2\left[\mathrm{SL}_{2}(3)\right]_{1}\end{array}$ & $\mathfrak{p}_{2} \mathfrak{p}_{11} F$ & $\infty, 2\left[\mathrm{SL}_{2}(11)\right]_{5} \circ C_{3}$ \\
\hline
\end{tabular}
Further $\operatorname{Aut}_{\mathbb{Q}(\sqrt{-11})}\left(L, \mathfrak{p}_{2} \mathfrak{p}_{3} \mathfrak{p}_{11}^{2 c-1} F\right)=\operatorname{Aut}_{\mathbb{Q}(\sqrt{-3})}\left(L, \mathfrak{p}_{2} \mathfrak{p}_{3} \mathfrak{p}_{11}^{2 c-1} F\right)$ for all $c$. The remaining 9 automorphism groups are given below.

\subsubsection{Proof of Theorem 4.11.1}

Suppose that $G<\operatorname{Sp}_{20}(\mathbb{Q})$ is s.p.i.m.f.. Before we discuss all possible Fitting subgroups of $G$, we describe all groups $G$ that contain an irreducible quasisimple normal subgroup.

\section{Lemma 4.11.5}

(a) If $G$ contains a normal subgroup $N \simeq{ }_{\infty, 2}\left[\mathrm{SL}_{2}(11)\right]_{5}$ then $G$ is conjugate to $\infty, 2\left[\mathrm{SL}_{2}(11)\right]_{5} \circ C_{3}$ or $\infty, 2\left[\mathrm{SL}_{2}(11)\right]_{5} \stackrel{2(2)}{\square} C_{4}$.

(b) If $G$ contains a normal subgroup $N \simeq{ }_{\infty, 2}\left[ \pm U_{5}(2)\right]_{5}$ then $G$ is conjugate to $\infty, 2\left[ \pm U_{5}(2)\right]_{5} \circ C_{3}, \infty, 2\left[ \pm U_{5}(2)\right]_{5} \stackrel{2(2)}{\circ} C_{4}$ or $\sqrt{-2}\left[\infty, 2\left[ \pm U_{5}(2)\right]_{5}: 2\right]_{10}$. 
Proof: In both cases, $N$ is rationally irreducible and has $\mathcal{Q}_{\infty, 2}$ as commuting algebra. By Remark 2.2.17, $G$ contains a subgroup $U$ conjugate to $\pm N: 2$ or $\pm N \circ C_{i}$ with $i \in\{3,4\}$. The commuting algebra of $U$ is $\mathbb{Q}(\sqrt{-d})$ with $d=2,3,1$ respectively. One easily checks that $\infty, 2\left[\mathrm{SL}_{2}(11)\right]_{5} \circ C_{3}, \infty, 2\left[ \pm U_{5}(2)\right]_{5} \circ C_{3}$ and $\sqrt{-2}\left[\infty, 2\left[ \pm U_{5}(2)\right]_{5}: 2\right]_{10}$ are s.i.m.f.. Further $\infty, 2\left[ \pm U_{5}(2)\right]_{5} \circ C_{4}$ fixes only one lattice and is only contained in $\infty, 2\left[ \pm U_{5}(2)\right]_{5}{ }^{2(2)} C_{4}$. The group $\infty, 2\left[\mathrm{SL}_{2}(11)\right]_{5}: 2$ fixes 4 lattices and is only contained in $\sqrt{-2}\left[2 . M_{12}: 2\right]_{10}$. Finally $\infty, 2\left[\mathrm{SL}_{2}(11)\right]_{5} \circ C_{4}$ fixes 2 lattices and is only contained in $\infty, 2\left[\mathrm{SL}_{2}(11)\right]_{5} \stackrel{2(2)}{\square} C_{4}$.

Lemma 4.11.6 $O_{11}(G)$ is trivial.

Proof: Suppose $O_{11}(G) \neq 1$. Then $O_{11}(G) \simeq C_{11}$ and it centralizer embeds into $\mathrm{GL}_{2}\left(\mathbb{Q}\left(\zeta_{11}\right)\right)$. In particular $E(G)=1$ and therefore $F(G)$ is self centralizing. If $F(G) \neq \pm O_{11}(G)$ then $G$ contains an irreducible cyclic subgroup of order 33 or 44 and $\Pi(|G|) \subseteq\{2,3,5,11\}$. This contradicts Lemmas 4.11.4 and 4.11.3. So $F(G) \simeq C_{22}$ and $[G: F(G)] \in\{5,10\}$ by Lemma 2.2.1. The group $C_{22}: C_{5}$ has only one faithful rational irreducible representation. It is of degree 10 and has $\mathbb{Q}(\sqrt{-11})$ as commuting algebra. This again contradicts Lemma 2.2.1.

Lemma 4.11.7 If $O_{5}(G)$ is nontrivial then $G$ is conjugate to $\zeta_{10}\left[ \pm 5_{+}^{1+2} \cdot \operatorname{Sp}_{2}(5)\right]_{5}$ or $\zeta_{10}\left[C_{10}\right]_{1} \otimes A_{5}$.

Proof: By Table 2.5.2, $O_{5}(G)$ is conjugate to $C_{25}, C_{5}$ or $5_{+}^{1+2}$. The first case contradicts Lemma 4.11.2. The irreducible group $\mathcal{B}^{o}\left(5_{+}^{1+2}\right)={ }_{\zeta_{10}}\left[ \pm 5_{+}^{1+2} \cdot \operatorname{Sp}_{2}(5)\right]_{5}$ fixes 2 lattices and has $\mathbb{Q}\left(\zeta_{10}\right)$ as commuting algebra. One easily checks that it is s.i.m.f.. So we may now assume that $O_{5}(G)=C_{5}$. Then $F(G)= \pm C_{5}$ and $E(G) \neq 1$ by Corollary 2.2.3. Thus $E(G)$ embeds into $\mathrm{GL}_{5}\left(\mathbb{Q}\left(\zeta_{10}\right)\right)$. It follows from Table 2.5.1 that $E(G)$ is conjugate to $\mathrm{Alt}_{6}$. Therefore $G$ contains a normal subgroup $N:=\mathcal{B}^{\circ}\left(F^{*}(G)\right) \simeq \zeta_{10}\left[C_{10}\right]_{1} \otimes A_{5}$. Since $N$ fixes 4 lattices and has $\mathbb{Q}\left(\zeta_{10}\right)$ as commuting algebra, one easily checks that $N$ is s.i.m.f..

Lemma 4.11.8 If $O_{3}(G) \neq 1$ then $G$ is conjugate to one of

$$
\begin{gathered}
\sqrt{-3}\left[ \pm S_{4}(3) \circ C_{3}\right]_{5} \underset{\sqrt{-3}}{\otimes}{ }_{\infty, 2}\left[\mathrm{SL}_{2}(3)\right]_{1},{ }_{i}\left[C_{4}\right]_{1} \otimes\left[C_{6} \times S_{4}(3) .2\right]_{10},{ }_{\infty, 2}\left[\mathrm{SL}_{2}(11)\right]_{5} \circ C_{3} \\
\infty, 2\left[ \pm U_{5}(2)\right]_{5} \circ C_{3}, \sqrt{-3}\left[ \pm U_{4}(2) \circ C_{3}\right]_{10}, \sqrt{-3}\left[ \pm \mathrm{L}_{2}(11) \stackrel{2(3)}{\otimes_{\sqrt{-3}}}\left[C_{6}\right]_{1}\right]_{10}, \sqrt{-11}\left[ \pm \mathrm{L}_{2}(11)\right]_{5} \otimes A_{2} \\
\sqrt{-3}\left[\sqrt{-11}\left[ \pm \mathrm{L}_{2}(11)\right]_{5} \stackrel{2(3)}{\otimes} \underset{\sqrt{-3}}{ }\left[C_{6}\right]_{1}\right]_{10} \text { or } \sqrt{-3}\left[C_{6}\right]_{1} \otimes H \text { with } H \in\left\{A_{10}, A_{10}^{(2)}, A_{10}^{(3)}\right\}
\end{gathered}
$$

Proof: If $O_{3}(G) \neq 1$ then $O_{3}(G)=C_{3}$. Thus $O_{2}(G) \in\left\{C_{2}, C_{4}, D_{8}, Q_{8}\right\}$ and $O_{p}(G)=1$ for all $p>3$. Let $N=\mathcal{B}^{\circ}\left(F^{*}(G)\right)$. 
- If $O_{2}(G) \simeq Q_{8}$ then $E(G)$ embeds into $G_{5}(\mathbb{Q}(\sqrt{-3}))$. By Table 2.5.1 $E(G)$ is conjugate to $\mathrm{Alt}_{6}$ or $S_{4}(3)$.

In the latter case $N \simeq \sqrt{-3}\left[ \pm S_{4}(3) \circ C_{3}\right]_{5} \underset{\sqrt{-3}}{\otimes} \infty, 2\left[\mathrm{SL}_{2}(3)\right]_{1}$ fixes 6 lattices and has $\mathbb{Q}(\sqrt{-3})$ as commuting algebra. One checks that it is s.i.m.f.. In the other case $N \simeq A_{5} \otimes \sqrt{-3}\left[\mathrm{SL}_{2}(3) \circ C_{3}\right]_{2}$ has the same endomorphism ring and fixes 18 lattices. One checks that it is only contained in $\sqrt{-3}\left[S_{4}(3) \circ C_{3}\right]_{5} \underset{\sqrt{-3}}{\otimes} \infty, 2\left[\mathrm{SL}_{2}(3)\right]_{1}$.

- If $O_{2}(G) \simeq C_{4}$ or $D_{8}$ then $E(G)$ embeds into $\mathrm{GL}_{5}\left(\mathbb{Q}\left(\zeta_{12}\right)\right)$. Thus again $E(G)$ is conjugate to $A_{5}$ or $S_{4}(3)$. The group $N_{1}:=A_{5} \otimes C_{12}$ fixes 9 lattices, but using part (a) of Remark 2.2 .8 one finds a normal critical lattice $L$. Let $F \in \mathcal{F}_{>0}\left(N_{1}\right)$, then $\operatorname{Aut}_{\mathbb{Q}\left(\zeta_{12}\right)}(L, F)=\sqrt{-3}\left[ \pm S_{4}(3) \circ C_{3}\right]_{5} \otimes C_{4}$. So $E(G) \simeq S_{4}(3)$ and $G$ must contain a normal subgroup conjugate to $N_{2}:=\sqrt{-3}\left[ \pm S_{4}(3) \circ C_{3}\right]_{5} \otimes C_{4}$. The group $N_{2}$ fixes 3 lattices but there exists a normal critical one, say $L$. Further there exists some $F \in \mathcal{F}_{>0}(N)$ that is integral on $L$ such that $\operatorname{det}(L, F)=2^{10}$ and $\Pi(|G|)=$ $\{2,3,5\}$. The maximal totally real subfield $K$ of $C:=\operatorname{End}\left(\overline{N_{2}}\right) \simeq \mathbb{Q}\left(\zeta_{12}\right)$ is isomorphic to $\mathbb{Q}(\sqrt{3})$. Since $\operatorname{End}_{\mathbb{Z} N_{2}}(L)$ is the maximal order in $C$ and since $\operatorname{Nr}_{C / K}\left(\mathbb{Z}_{C}^{*}\right)=\mathbb{Z}_{K,>0}^{*}$ we may restrict ourselves to one class of totally positive units. Thus Table 2.5.4 shows that there are 4 candidates to check:

\begin{tabular}{|c|c|c|}
\hline form & $\mathbb{Q}(i)$ & $\mathbb{Q}(\sqrt{-3})$ \\
\hline \hline$F$ & $\infty, 2\left[ \pm U_{5}(2)\right]_{5}{ }^{2(2)} C_{4}$ & $\sqrt{-3}\left[ \pm S_{4}(3) \circ C_{3}\right]_{5} \underset{\sqrt{-3} \infty, 2}{\otimes}\left[\mathrm{SL}_{2}(3)\right]_{1}$ \\
\hline $\mathfrak{p}_{2}^{-1} \mathfrak{p}_{3} F$ & ${ }_{i}\left[C_{4}\right]_{1} \otimes\left[C_{6} \times S_{4}(3) .2\right]_{10}$ & $\lesseqgtr \sqrt{-3}\left[ \pm S_{4}(3) \circ C_{3}\right]_{5}^{2}$ \\
\hline
\end{tabular}

Only ${ }_{i}\left[C_{4}\right]_{1} \otimes\left[C_{6} \times S_{4}(3) .2\right]_{10}$ has the correct Fitting subgroup.

- If $O_{2}(G)= \pm 1$, then $E(G)$ is isomorphic to $\operatorname{Alt}_{6}, S_{4}(3)=U_{4}(2)$ (2 representations each), $\operatorname{Alt}_{11}, \mathrm{~L}_{2}(11)$ (3 representations), $\mathrm{SL}_{2}(11)$ or $U_{5}(2)$.

If $E(G) \simeq \mathrm{SL}_{2}(11)$ or $U_{5}(2)$ then $G=E(G) \circ C_{3}$ by Lemma 4.11.5.

If $E(G) \simeq$ Alt $_{11}$ then $N \simeq \sqrt{-3}\left[C_{6}\right]_{1} \otimes A_{10}$ fixes 2 lattices and is already s.i.m.f..

If $E(G) \simeq U_{4}(2)$ with character $\chi_{10 a b}$ then $N \simeq \sqrt{-3}\left[ \pm U_{4}(2) \circ C_{3}\right]_{10}$ is already irreducible and fixes 2 lattices. One checks that it is s.i.m.f..

If $E(G) \simeq U_{4}(2)$ with character $2 \chi_{5 a b}$ then $N$ is conjugate to $\sqrt{-3}\left[ \pm S_{4}(3) \circ C_{3}\right]_{5}$ and $[G: N]=4$ by Lemma 2.2.1. In particular, $G$ must contain a normal subgroup conjugate to $S_{4}(3) \otimes A_{2}$ or $\pm S_{4}(3) \underset{\sqrt{-3}}{\otimes} \infty, 3\left[\tilde{S}_{3}\right]_{1}$. These two groups fix 15 and 5 lattices respectively. They are only contained in $\left.\sqrt{-3}\left[ \pm S_{4}(3) \circ C_{3}\right)\right]_{5}^{2}$.

If $E(G) \simeq$ Alt $_{6}$ with character $4 \chi_{5 a}$, then $N \simeq \sqrt{-3}\left[C_{6}\right]_{1} \otimes A_{5} \simeq{ }_{\sqrt{-3}}\left[C_{6}\right]_{1} \otimes S_{6}$. Since the exceptional outer automorphism of Alt 6 interchanges its 5 dimensional irreducible characters, we have $[G: N] \leq 2$. This contradicts Lemma 2.2.1.

If $E(G) \simeq \mathrm{Alt}_{6}$ with character $2 \chi_{10}$, then $N \simeq \sqrt{-3}\left[C_{6}\right]_{1} \otimes\left[ \pm S_{6}\right]_{10}$ is already irreducible and fixes 12 lattices. One checks that it is only contained in $\sqrt{-3}\left[ \pm U_{4}(2) \circ C_{3}\right]_{10}$.

If $E(G) \simeq \mathrm{L}_{2}(11)$ with character $2 \chi_{10 a}$, then $N \simeq \sqrt{-3}\left[C_{6}\right]_{1} \otimes A_{10}^{(2)}$ has $\mathbb{Q}(\sqrt{-3})$ as commuting algebra and fixes 2 lattices. One checks that it is s.i.m.f.. 
If $E(G) \simeq \mathrm{L}_{2}(11)$ with character $2 \chi_{10 b}$, then $N=F^{*}(G)$ has $\mathbb{Q}(\sqrt{-3})$ as commuting algebra and fixes 6 lattices. One checks that it is only contained in $\sqrt{-3}\left[C_{6}\right]_{1} \otimes A_{10}$ and $\sqrt{-3}\left[ \pm \mathrm{L}_{2}(11)^{2(3)} \otimes_{\sqrt{-3}}\left[C_{6}\right]_{1}\right]_{10}$.

If $E(G) \simeq \mathrm{L}_{2}(11)$ with character $2 \chi_{5 a b}$, then $F^{*}(G)$ contains an irreducible cyclic subgroup of order 33 and $|G|=\{2,3,5,11\}$. It follows from Lemma 4.11.4 that $G$ is conjugate to $\sqrt{-3}\left[\sqrt{-11}\left[ \pm \mathrm{L}_{2}(11)\right]_{5} \stackrel{2(3)}{\otimes} \sqrt{-3}\left[C_{6}\right]_{1}\right]_{10}, \quad \sqrt{-3}\left[C_{6}\right]_{1} \otimes A_{10}^{(3)}$ or $\sqrt{-11}\left[ \pm \mathrm{L}_{2}(11)\right]_{5} \otimes A_{2}$.

Lemma 4.11.9 If $F(G) \simeq D_{8} \otimes C_{4}$ then $G$ is conjugate to ${ }_{i}\left[\left(D_{8} \otimes C_{4}\right) . S_{3}\right]_{2} \otimes A_{5}$.

Proof: Since $E(G)$ embeds into $\mathrm{GL}_{5}(\mathbb{Q}(i))$, it is conjugate to $\operatorname{Alt}_{6}$. In particular $G$ contains the normal subgroup $N:=\mathcal{B}^{\circ}\left(F^{*}(G)\right) \simeq_{i}\left[\left(D_{8} \otimes C_{4}\right) \cdot S_{3}\right]_{2} \otimes A_{5}$. The group $N$ fixes 6 lattices and is easily checked to be s.i.m.f..

Lemma 4.11.10 $F(G) \not D_{8} \otimes D_{8}$.

Proof: By Table 2.5.1, $E(G) \simeq$ Alt $_{6}$. But then $F^{*}(G)$ is already irreducible and fixes no skewsymmetric form.

Lemma 4.11.11 If $F(G) \simeq Q_{8}$, then $G$ is conjugate to $\sqrt{-11}\left[\mathrm{~L}_{2}(11)\right]_{5} \underset{\sqrt{-11}}{\otimes} \infty_{, 2}\left[\mathrm{SL}_{2}(3)\right]_{1}$ or $\sqrt{-2}\left[\mathrm{GL}_{2}(3)\right]_{2} \otimes A_{5}$.

Proof: Since $E(G)$ embeds into $\mathrm{GL}_{5}\left(\mathcal{Q}_{\infty, 2}\right)$, it is conjugate to $\operatorname{Alt}_{6}$ or $\mathrm{L}_{2}(11)$ by Table 2.5.1 (note that $\left.O_{3}\left(\mathcal{B}^{o}\left(S_{4}(3)\right)\right)=C_{3}\right)$.

In the latter case $\mathcal{B}^{o}\left(F^{*}(G)\right) \simeq \sqrt{-11}\left[\mathrm{~L}_{2}(11)\right]_{5} \underset{\sqrt{-11}}{\otimes} \infty, 2\left[\mathrm{SL}_{2}(3)\right]_{1}$ is already s.i.m.f.. In the first case $N:=\mathcal{B}^{\circ}\left(F^{*}(G)\right) \simeq_{\infty, 2}\left[\mathrm{SL}_{2}(3)\right]_{1} \otimes A_{5}$ is irreducible and has $\mathcal{Q}_{\infty, 2}$ as commuting algebra. So by Remark 2.2.17, $G$ must contain a subgroup conjugate to $N .2$ or $N \circ C_{i}$ with $i \in\{3,4\}$. These groups fix 12,18 and 6 lattices respectively. One checks that $N: 2 \simeq \sqrt{-2}\left[\mathrm{GL}_{2}(3)\right]_{2} \otimes A_{5}$ is already s.i.m.f.. The other two groups are properly contained in $\sqrt{-3}\left[ \pm S_{4}(3) \circ C_{3}\right]_{5} \underset{\sqrt{-3}}{\otimes} \infty, 2\left[\mathrm{SL}_{2}(3)\right]_{1}$ or ${ }_{i}\left[\left(D_{8} \otimes C_{4}\right) . S_{3}\right]_{2} \otimes A_{5}$ respectively.

Lemma 4.11.12 $F(G)$ is not conjugate to $C_{8}, D_{16}$ or $Q D_{16}$.

Proof: In any case $F(G)$ would contain a cyclic characteristic subgroup of order 8 . Thus $E(G)$ embeds into $\mathrm{GL}_{5}\left(\mathbb{Q}\left(\zeta_{8}\right)\right)$ and must be conjugate to $\mathrm{Alt}_{6}$. So $G$ contains a normal subgroup $N \simeq C_{8} \otimes A_{5}$. Since the exceptional outer automorphism of $\mathrm{Alt}_{6}$ interchanges its absolutely irreducible 5-dimensional characters, we have $G / N \leq \operatorname{Out}\left(C_{8}\right) \simeq C_{2} \times C_{2}$. In particular $\Pi(|G|)=\{2,3,5\}$. The group $N$ fixes 20 lattices. By looking at the indices of these lattices in the standard lattice, one sees 
that $L=\mathbb{Z}^{1 \times 20}$ is normal critical and there exists some integral $F \in \mathcal{F}_{>0}(N)$ such that $\operatorname{det}(L, F)=2^{4} \cdot 3^{4}$. The maximal totally real subfield of $\mathbb{Q}\left(\zeta_{8}\right)$ is isomorphic to $\mathbb{Q}(\sqrt{2})$. So Table 2.5.4 shows that there are 4 groups to check:

\begin{tabular}{|c|c|c|}
\hline form & $\mathbb{Q}(i)$ & $\mathbb{Q}(\sqrt{-2})$ \\
\hline \hline$F$ & $\left({ }_{i}\left[C_{4}\right]_{1} \otimes A_{5}\right)^{2}$ & $\subsetneq \sqrt{-2}\left[\mathrm{GL}_{2}(3)\right]_{2} \otimes A_{5}$ \\
\hline $\mathfrak{p}_{2} F$ & ${ }_{i}\left[\left(D_{8} \otimes C_{4}\right) . S_{3}\right]_{2} \otimes A_{5}$ & $\sqrt{-2}\left[\mathrm{GL}_{2}(3)\right]_{2} \otimes A_{5}$ \\
\hline
\end{tabular}

None of these has the correct Fitting subgroup.

\section{Lemma 4.11.13}

(a) If $F(G) \simeq C_{4}$, then $G$ is conjugate to $\infty, 2\left[ \pm U_{5}(2)\right]_{5} \stackrel{2(2)}{\circ} C_{4}, \infty, 2\left[\mathrm{SL}_{2}(11)\right]_{5} \stackrel{2(2)}{\square} C_{4}$, ${ }_{i}\left[\left[ \pm S_{6}\right]_{10} \stackrel{2(2)}{\otimes}{ }_{i}\left[C_{4}\right]_{1}\right]_{10}$ or ${ }_{i}\left[C_{4}\right]_{1} \otimes H$ with $H \in\left\{A_{10}, A_{10}^{(2)}, A_{10}^{(3)}\right\}$.

(b) $F(G)$ is not conjugate to $D_{8}$.

Proof: In both cases, $F(G)$ contains a characteristic normal cyclic subgroup $U$ of order 4. Thus $E(G)$ embeds into $\mathrm{GL}_{10}(\mathbb{Q}(i))$. By Table 2.5.1, $E(G)$ is conjugate to $\mathrm{Alt}_{6}$ (2 representations), $\mathrm{L}_{2}(11)$ (3 representations), $\mathrm{Alt}_{11}, \mathrm{SL}_{2}(11)$ or $U_{5}(2)$ (note again that $\left.O_{3}\left(\mathcal{B}^{\circ}\left(S_{4}(3)\right)\right)=C_{3}\right)$. The groups $U_{5}(2)$ and $\mathrm{SL}_{2}(11)$ have been handled in Lemma 4.11.5. Let $N=\mathcal{B}^{o}(E(G) U)$.

- If $E(G) \simeq \operatorname{Alt}_{11}$, then $N \simeq{ }_{i}\left[C_{4}\right]_{1} \otimes A_{10}$ fixes 2 lattices and has $\mathbb{Q}(i)$ as commuting algebra. It is already s.i.m.f..

- If $E(G) \simeq \operatorname{Alt}_{6}$ with character $2 \chi_{10}$, then $N$ has $\mathbb{Q}(i)$ as commuting algebra and fixes 22 lattices. It is only contained in ${ }_{i}\left[\left[ \pm S_{6}\right]_{10} \stackrel{2(2)}{\otimes}{ }_{i}\left[C_{4}\right]_{1}\right]_{10}$.

- Suppose $E(G) \simeq \mathrm{Alt}_{6}$ with character $4 \chi_{5 a}$. Since the exceptional outer automorphism of Alt $_{6}$ interchanges its absolutely irreducible 5-dimensional characters and $\mathcal{B}^{\circ}(E(G)) \simeq A_{5}$, we get that $G / N \leq \operatorname{Out}(F(G))$. In particular $F(G)=D_{8}$ by Lemma 2.2.1. Let $\triangle(N)$ be a summand of the natural representation of $N$. Then $\triangle(N)<\mathrm{GL}_{10}(\mathbb{Q})$ is absolutely irreducible and has $\mathbb{Q}$ as commuting algebra. So $G$ embeds into $\mathrm{GL}_{10}(K)$ for some imaginary quadratic number field and $\triangle(N)$ is an irreducible subgroup of $G<\mathrm{GL}_{10}(K)$. One easily checks that only the outer automorphism acting on $D_{8}$ and fixing $A_{5}$ can be realized in $\mathrm{GL}_{10}(\mathbb{C})$. Only the non-split extension is symplectic, since it can be realized over $\mathbb{Q}(\sqrt{-2})$. It fixes 40 lattices and is only contained in $\sqrt{-2}\left[\mathrm{GL}_{2}(3)\right]_{2} \otimes A_{5}$.

- If $E(G) \simeq \mathrm{L}_{2}(11)$ with character $2 \chi_{10 a}$, then $N \simeq_{i}\left[C_{4}\right]_{1} \otimes A_{10}^{(2)}$ has $\mathbb{Q}(i)$ as commuting algebra and fixes 2 lattices. One checks that it is s.i.m.f..

- If $E(G) \simeq \mathrm{L}_{2}(11)$ with character $2 \chi_{10 b}$, then $N=F^{*}(G)$ has $\mathbb{Q}(i)$ as commuting algebra and fixes 4 lattices. It is only contained in ${ }_{i}\left[C_{4}\right]_{1} \otimes A_{10}$. 
- If $E(G) \simeq \mathrm{L}_{2}(11)$ with character $2 \chi_{5 a b}$, then $N=F^{*}(G)$ contains an irreducible cyclic subgroup of order 44 and $\Pi(|G|)=\{2,3,5,11\}$. Thus $G$ is conjugate to ${ }_{i}\left[C_{4}\right]_{1} \otimes A_{10}^{(3)}$ by Lemma 4.11 .3 .

Lemma 4.11.14 If $F(G)= \pm I_{20}$, then $G$ is conjugate to one of

$$
\sqrt{-19}\left[\mathrm{SL}_{2}(19)\right]_{10}, \sqrt{-2}\left[\infty, 2\left[ \pm U_{5}(2)\right]: 2\right]_{10}, \sqrt{-2}\left[2 . M_{12}: 2\right]_{10}, \sqrt{-7}\left[2 . M_{22}: 2\right]_{10} .
$$

Proof: By Table 2.5.1, $E(G)$ is isomorphic to $\mathrm{Alt}_{6}$ (2 representations), $\mathrm{L}_{2}(11)$ (3 representations), $\mathrm{Alt}_{11}, \mathrm{SL}_{2}(11), \mathrm{Alt}_{7}, 2 . M_{12}, 2 . M_{22}, U_{5}(2), 2 . L_{3}(4)$ or $\mathrm{SL}_{2}(19)$. Note that $\mathrm{O}_{3}\left(\mathcal{B}^{\circ}\left(U_{4}(2)\right)\right)=C_{3}$ for $\chi_{5 a b}$ and $\chi_{10 a b}$.

The group $\sqrt{-19}\left[\mathrm{SL}_{2}(19)\right]_{10}$ is s.i.m.f. by Theorem 3.2.1. The cases $E(G) \simeq U_{5}(2)$ or $\mathrm{SL}_{2}(11)$ are handled in Lemma 4.11.5. Let $N=\mathcal{B}^{\circ}(E(G))$.

- If $E(G) \simeq 2 . M_{12}$ or $2 . M_{22}$ then $N= \pm E(G): 2$ fixes only one lattice and has $\mathbb{Q}(\sqrt{-2})$ or $\mathbb{Q}(\sqrt{-7})$ as commuting algebra. One checks that $N$ is s.i.m.f..

- If $E(G) \simeq \mathrm{Alt}_{7}$, then $N \simeq \pm \mathrm{Alt}_{7}$ fixes 5 lattices and has $\mathbb{Q}(\sqrt{-7})$ as commuting algebra. One checks that it is only contained in $\sqrt{-7}\left[2 . M_{22}: 2\right]_{10}$.

- If $E(G) \simeq 2 . L_{3}(4)$, then $N \simeq 2 . L_{3}(4) .2_{2}$ fixes 5 lattices and has $\mathbb{Q}(\sqrt{-7})$ as commuting algebra. One checks that it is only contained in $\sqrt{-7}\left[2 . M_{22}: 2\right]_{10}$.

- If $E(G) \simeq \operatorname{Alt}_{11}$, then $G / \pm \operatorname{Alt}_{11} \leq \operatorname{Out}\left(\operatorname{Alt}_{11}\right) \simeq C_{2}$ and $N \simeq A_{10} \simeq \pm S_{11}$. Thus $G=N$ is reducible.

- If $E(G) \simeq \mathrm{L}_{2}(11)$ with character $2 \chi_{5 a b}$ then $N=F(G)$. Hence $G / N \leq$ $\operatorname{Out}(N) \simeq C_{2}$ contradicts Lemma 2.2.1.

- If $E(G) \simeq \mathrm{L}_{2}(11)$ with character $2 \chi_{10 a}$, then $N \simeq \pm \mathrm{L}_{2}(11): 2$. Hence $G / \pm E(G) \leq$ $\operatorname{Out}\left(\mathrm{L}_{2}(11)\right) \simeq C_{2}$ implies that $G=N$ is reducible.

- If $E(G) \simeq \mathrm{L}_{2}(11)$ with character $2 \chi_{10 b}$, then $N=F^{*}(G)$ and again $[G: N]=2$. Since $\chi_{10 b}$ as absolutely irreducible, $G$ embeds into $\mathrm{GL}_{10}(K)$ for some imaginary quadratic number field $K$ such that $N<G$ is irreducible. One easily constructs $N \cdot 2<\mathrm{GL}_{10}(\mathbb{Q}(\sqrt{-3}))$. But $N \cdot 2$ cannot be s.i.m.f. since $\mathbb{Q}(\sqrt{-3})$ contains a third root of unity.

- If $E(G) \simeq$ Alt $_{6}$ with character $4 \chi_{5 a}$ then $N \simeq A_{5} \simeq \pm S_{6}$. Since the exceptional outer automorphism of $\operatorname{Alt}_{6}$ interchanges $\chi_{5 a}$ and $\chi_{5 b}$, we see that $G=N$ is reducible.

- If $E(G) \simeq \mathrm{Alt}_{6}$ with character $2 \chi_{10}$ then $N \simeq \pm S_{6}$ and $[G: N] \leq 2$. Again $G<\mathrm{GL}_{10}(K)$ for some imaginary quadratic number field $K$ such that $N<G$ is irreducible. One easily constructs $N: 2<\mathrm{GL}_{10}(\mathbb{Q}(\sqrt{-2}))$. But $N: 2$ is only contained in $\sqrt{-2}\left[2 . M_{12}: 2\right]_{10}$. 


\subsection{Dimension 22}

Theorem 4.12.1 The s.i.m.f. subgroups $G$ of $\operatorname{Sp}_{22}(\mathbb{Q})$ are

\begin{tabular}{|c|c|c|c|c|c|}
\hline$\#$ & $G$ & $|G|$ & $|\mathcal{Z}(G)|$ & $L_{\text {min }}$ & $\begin{array}{c}\text { r.i.m.f. } \\
\text { supergroups }\end{array}$ \\
\hline \hline$[2,1,11]$ & ${ }_{i}\left[C_{4}\right]_{1}^{11}$ & $2^{30} \cdot 3^{4} \cdot 5^{2} \cdot 7 \cdot 11$ & 3 & {$[1,1,44]$} & $B_{22}$ \\
\hline 1 & ${ }_{i}\left[C_{4}\right]_{1} \otimes A_{11}$ & $2^{12} \cdot 3^{5} \cdot 5^{2} \cdot 7 \cdot 11$ & 10 & {$\left[3^{2}, 2,264\right]$} & $A_{11}^{2}$ \\
\hline \hline$[2,2,11]$ & $\sqrt{-3}\left[C_{6}\right]_{1}^{11}$ & $2^{19} \cdot 3^{1} 5 \cdot 5^{2} \cdot 7 \cdot 11$ & 1 & {$\left[3^{11}, 2,66\right]$} & $A_{2}^{11}$ \\
\hline 2 & $\sqrt{-3}\left[C_{6}\right]_{1} \otimes A_{11}$ & $2^{11} \cdot 3^{6} \cdot 5^{2} \cdot 7 \cdot 11$ & 9 & {$\left[3^{11}, 4,396\right]$} & $A_{11} \otimes A_{2}$ \\
\hline 3 & $\sqrt{-3}\left[ \pm U_{5}(2) \circ C_{3}\right]_{11}$ & $2^{11} \cdot 3^{6} \cdot 5 \cdot 11$ & 4 & {$\left[2^{2} \cdot 3,4,49896\right]$} & $S$ \\
\hline \hline 4 & $\sqrt{-23}\left[ \pm \mathrm{L}_{2}(23)\right]_{11}$ & $2^{4} \cdot 3 \cdot 11 \cdot 23$ & 3 & $\begin{array}{c}{\left[23^{11}, 12,1012\right]} \\
{\left[23^{11}, 12,506\right]}\end{array}$ & $A_{22}^{(6)}$ \\
\hline
\end{tabular}

where $S:=\left[ \pm P S U_{6}(2) . S_{3}\right]_{22}<\mathrm{GL}_{22}(\mathbb{Q})$.

Proof: By explicit calculations, one verifies that the above table is correct and yields s.i.m.f. groups. Further the r.i.m.f. supergroups are easily constructed since all s.i.m.f. groups are uniform. It remains to show the completeness of the classification.

The group $\sqrt{-3}\left[C_{6}\right]_{1}^{11}$ is s.i.m.f. by Lemma 2.1 .21 and ${ }_{i}\left[C_{4}\right]_{1}^{11}$ fixes only three lattices. One checks that it is s.i.m.f.. So we may now suppose that $G$ is s.p.i.m.f..

It follows from Corollary 4.1 .2 that $E(G)$ is not trivial. Thus by Table 2.5.1, $E(G)$ is conjugate to $\mathrm{Alt}_{12}, M_{11}, U_{5}(2)$ or $\mathrm{L}_{2}(23)$. In the latter two cases, $E(G)$ is irreducible and $\mathcal{B}^{\circ}(E(G))$ is already s.i.m.f..

If $E(G) \simeq$ Alt $_{12}$ then $G$ contains a normal subgroup $B:=\mathcal{B}^{\circ}(E(G)) \simeq A_{11} \simeq \pm S_{12}$. Clearly $B$ is not self-centralizing. Thus $G$ contains an irreducible subgroup ${ }_{i}\left[C_{4}\right]_{1} \otimes A_{11}$ or $\sqrt{-3}\left[C_{6}\right]_{1} \otimes A_{11}$. Both groups are s.i.m.f..

If $E(G) \simeq M_{11}$ then $\operatorname{Out}(E(G))$ is trivial. Thus $G$ contains an irreducible subgroup conjugate to ${ }_{\sqrt{-3}}\left[C_{6}\right]_{1} \otimes M_{11}$ or ${ }_{i}\left[C_{4}\right]_{1} \otimes M_{11}$. The first group fixes 18 lattices and is only contained in $\sqrt{-3}\left[C_{6}\right]_{1} \otimes A_{11}$ and $\sqrt{-3}\left[C_{6}\right]_{1}^{11}$. The second group fixes 15 lattices and is only contained in ${ }_{i}\left[C_{4}\right]_{1} \otimes A_{11}$ and ${ }_{i}\left[C_{4}\right]_{1}^{11}$. 


\section{Appendix A}

\section{Invariant Forms}

For each conjugacy class of s.p.i.m.f. matrix groups in $\mathrm{GL}_{2 n}(\mathbb{Q})$ where $1 \leq n \leq 11$ we give a symmetric positive definite form $F$ and a skewsymmetric form $S$ such that

$$
\operatorname{Aut}\left(\mathbb{Z}^{1 \times 2 n},\{F, S\}\right)=\left\{g \in \mathrm{GL}_{2 n}(\mathbb{Z}) \mid g F g^{\mathrm{tr}}=F \text { and } g S g^{\mathrm{tr}}=S\right\}
$$

is a representative of that class.

Further the pairs $(F, S)$ satisfy the following properties:

(a) $\operatorname{det}(F)=\min \left\{\operatorname{det}\left(L, F^{\prime}\right) \mid\left(L, F^{\prime}\right) \in \mathcal{Z}(G) \times \mathcal{F}_{>0}(G)\right.$ is integral $\}$ where $G:=\operatorname{Aut}\left(\mathbb{Z}^{1 \times 2 n},\{F, S\}\right)$.

(b) $S \cdot F^{-1}$ generates the commuting algebra of $\operatorname{Aut}\left(\mathbb{Z}^{1 \times 2 n},\{F, S\}\right)$ and its minimal polynomial $\mu\left(S \cdot F^{-1}, X\right)$ is one of

- $X^{2}+d$ for some squarefree $d \in \mathbb{N}$.

- $\mu\left(\zeta_{k}-\zeta_{k}^{-1}, X\right)$ for some even $k \in \mathbb{Z}_{\geq 6}$.

- $\mu\left(\zeta_{26}+\zeta_{26}^{3}+\zeta_{26}^{9}, X\right)$.

- $\mu\left(\sqrt{k} \cdot\left(\zeta_{\ell}-\zeta_{\ell}^{-1}\right), X\right)$ where $(k, \ell) \in\{(2,10),(3,10),(3,16)\}$.

- $\mu(i+\sqrt{-3}+\sqrt{-5}, X)$.

By Algorithm 2.3.3, these pairs $(F, S)$ give an easy way to recover the conjugacy class of any given s.p.i.m.f. subgroup of $\mathrm{GL}_{2 n}(\mathbb{Q})$ for $1 \leq n \leq 11$.

In some cases, tensoring such a pair $(F, S)$ with a gram matrix $g$ of some root lattice yields another s.p.i.m.f. automorphism group $H$. If the pair $(F \otimes g, S \otimes g)$ satisfies property (a) from above, then $H$ is omitted in the list below, since these forms can easily be reconstructed from the name of $H$.

For example, tensoring the forms of ${ }_{i}\left[C_{4}\right]_{1}$ with any gram matrix of $A_{2}$ yields a pair of forms that satisfies the above properties. Thus the s.p.i.m.f. matrix group ${ }_{i}\left[C_{4}\right]_{1} \otimes A_{2}$ is omitted in the list below.

Similarly, tensoring the forms of ${ }_{i}\left[C_{4}\right]_{1}$ with any gram matrix of $A_{5}$ yields a pair of forms whose automorphism group is conjugate to ${ }_{i}\left[C_{4}\right]_{1} \otimes A_{5}$. But these forms do not satisfy the above properties. Thus the list below contains some other forms for ${ }_{i}\left[C_{4}\right]_{1} \otimes A_{5}$. 

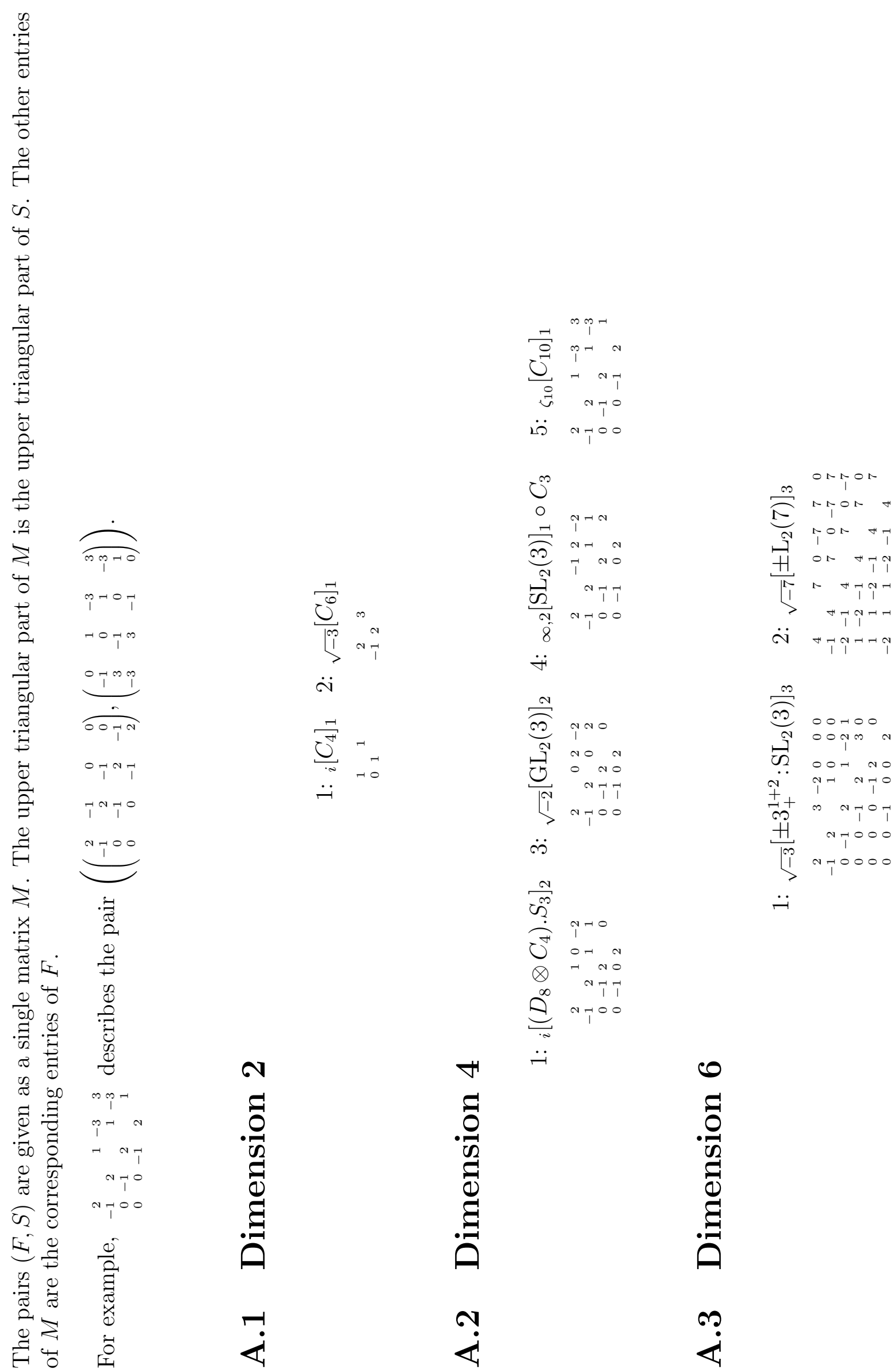

N
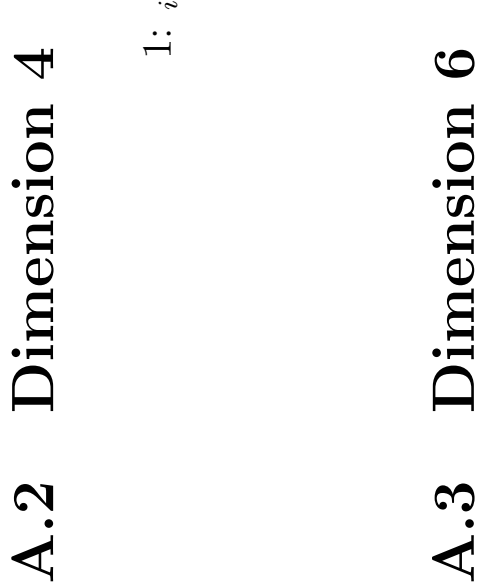

$\underset{1}{r}$ 

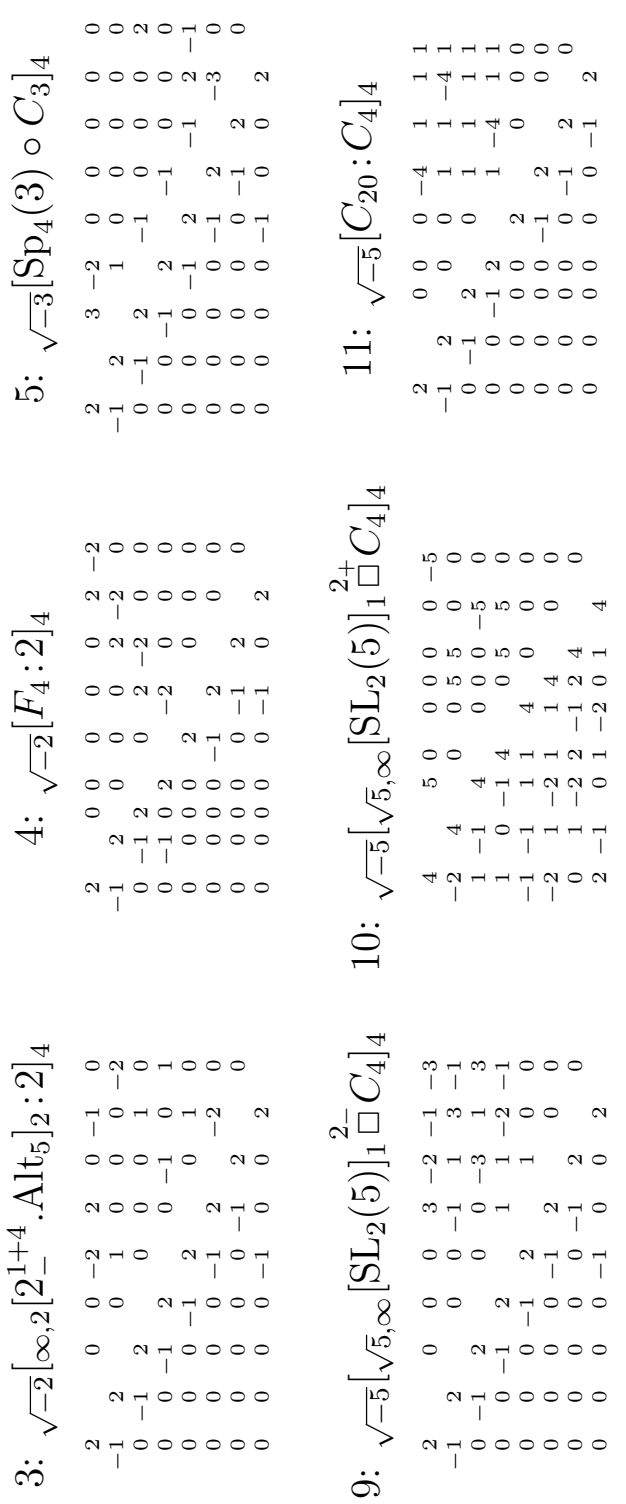

$\ddot{\sigma}$

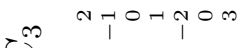

H. in too

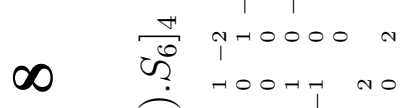

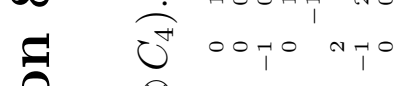

- $\frac{1}{2} \otimes 000$ n

مै

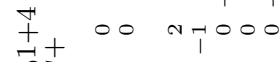

IN $0 N-10000$

h

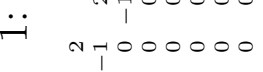

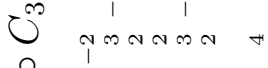

$\frac{N}{0} 0 T_{0}^{1}$

तิ

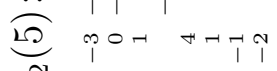

๑ึ

$\frac{8}{\pi}$

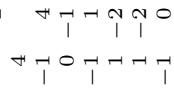

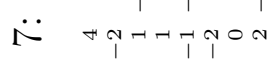

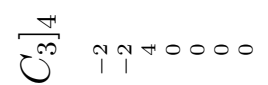
n

F nOONO no

I

8 oOd nhoo

IN 00 noOdo

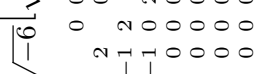

iे N

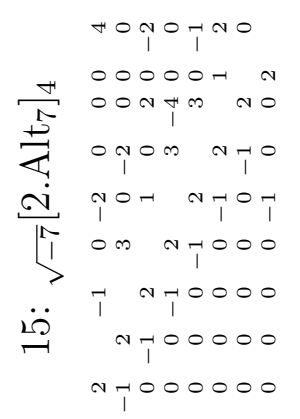

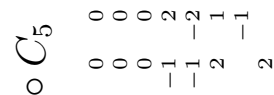

r

[2 $0000 \mathrm{n} / 0$

กู

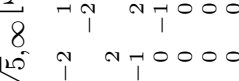

$\therefore \quad N H 00000$

$\ddot{\sigma} N=00000$

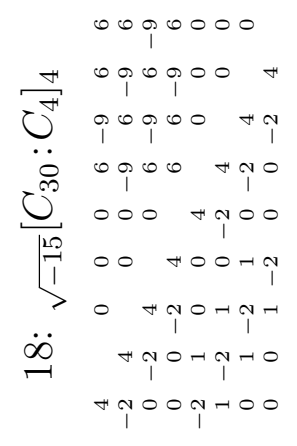

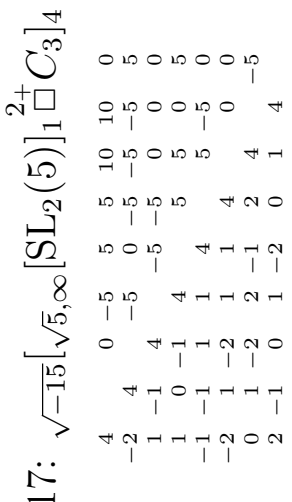

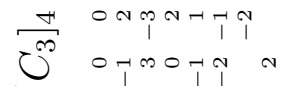

$\overbrace{}^{\infty} 00001000$

ND OnNat no

iص

8 oht nho

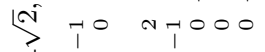

$\int_{i}^{1}$ m n n h $n H 00000$

$\ddot{\sim} N 1000000$
ND i hoONm n

ID ONOOH no

$\underbrace{-15}_{n}$ noom nho

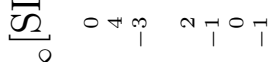

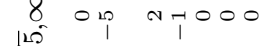

$\frac{1}{30}-N-10000$

I N N N

$\ddot{\theta}$ 

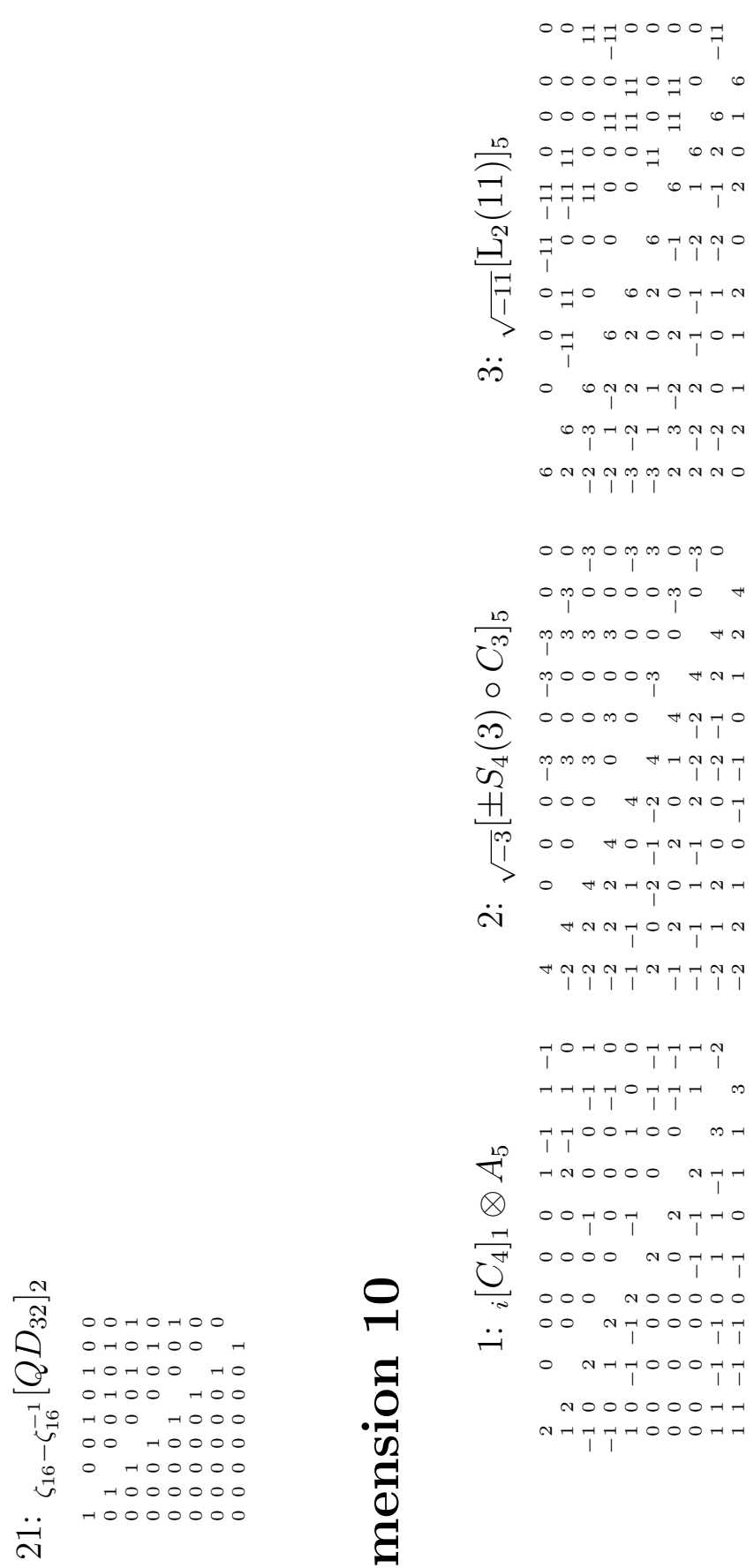

1
$H$

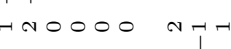

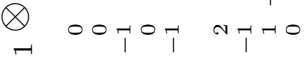

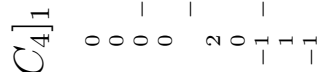

- 000 no0000

o o n h

o nht, 400,4

nOOOOOOH

$4 h t h-10004 \pi$
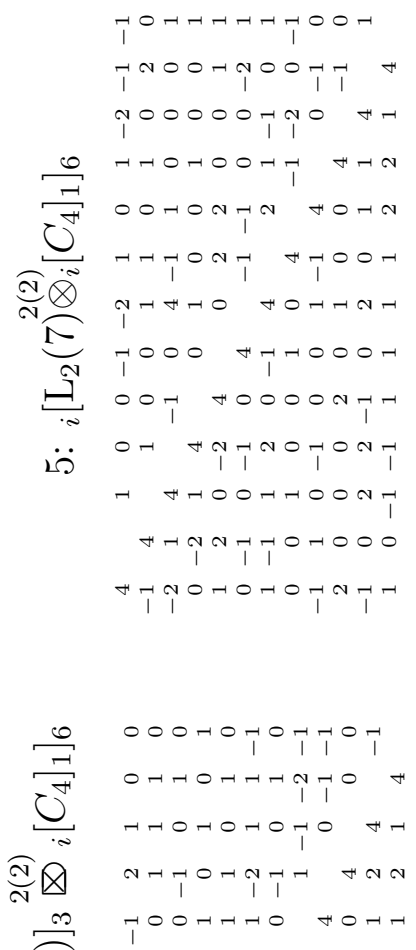

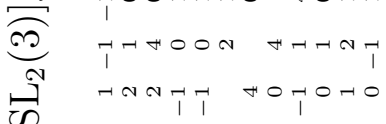

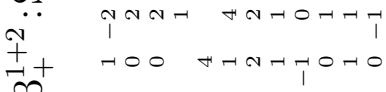

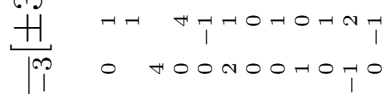

$4 h 000404740$

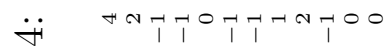

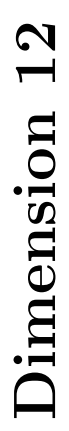

J $0000 \frac{1}{1} 0^{1}$

$m_{m}^{1}$
$m$
0
0

OHOOH haNOTO

OOHO htin hin

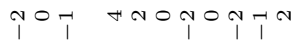

i.

$\ddot{\sim}$

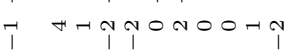

HONHTNNOONO

HOH HONOH hO 

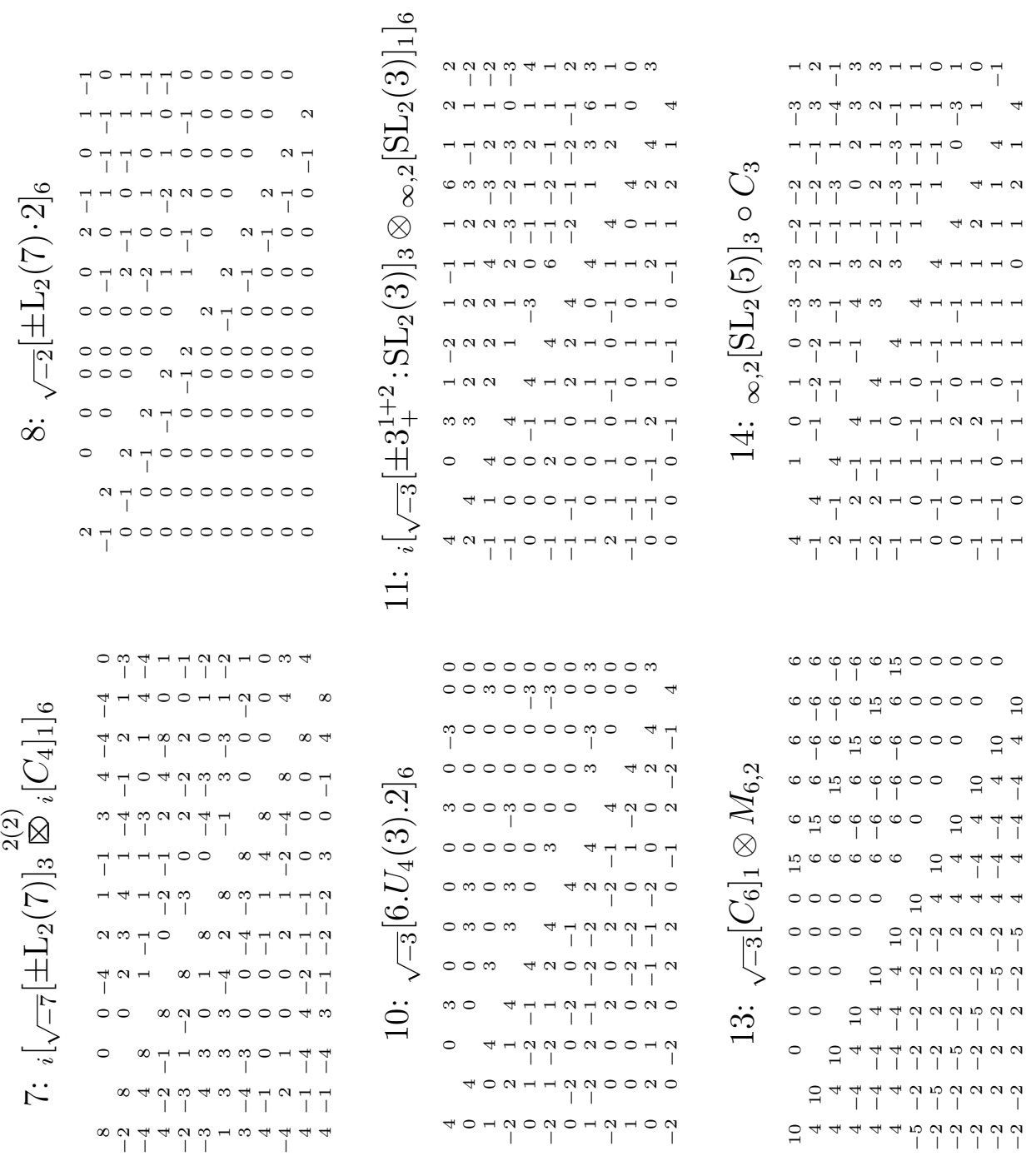

000 0 0000000

0000200000 욕 $000200000 \%$

ֻู

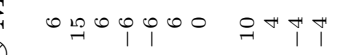
$\otimes$

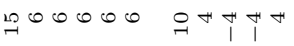
$00000 \rho_{H}$ o OOD O

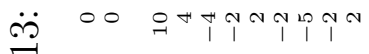

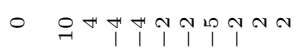
$\begin{array}{ccc}1 & 1 \\ 0 & 1 & 1 \\ 4 & 1 & 1\end{array}$

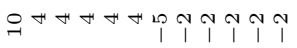

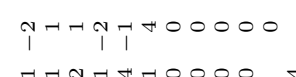

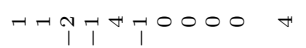
$A T+4 \hat{T}$

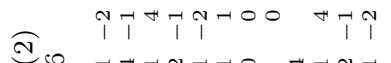

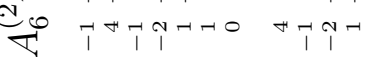

8 Hi

H 00000 H h 0000 H000000 000 H 1000000

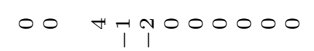
$0 \quad A h i, h 000000$

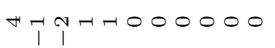
$A T h H \rightarrow h 00,10000$

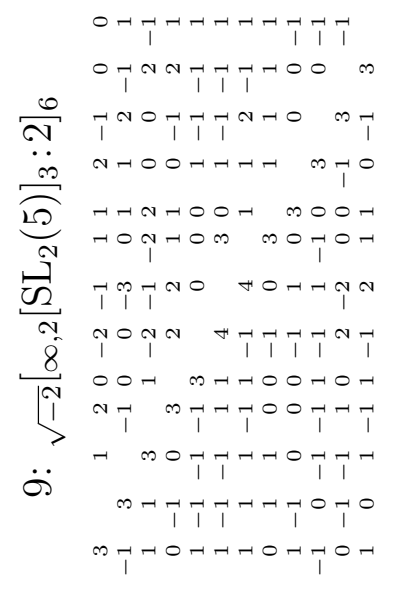

O nOTOMn moomonmmpi $0 p_{1} 00000 p_{1} \infty$

O mp omm $H$ p poo

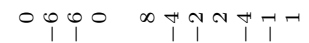
om $\dot{0}$ oO $\infty \pi m^{1}$

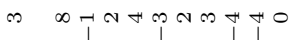
nO

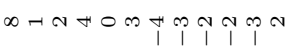




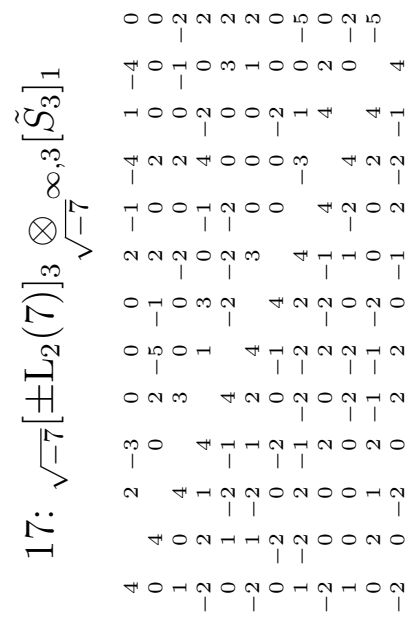

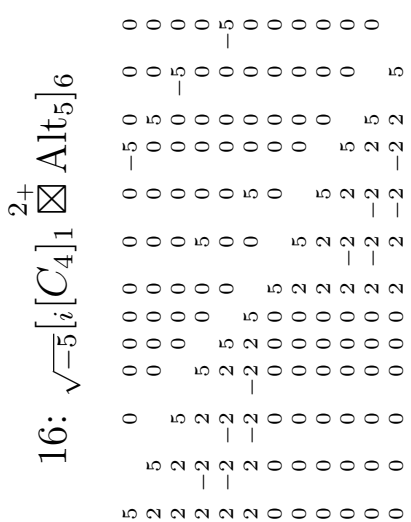

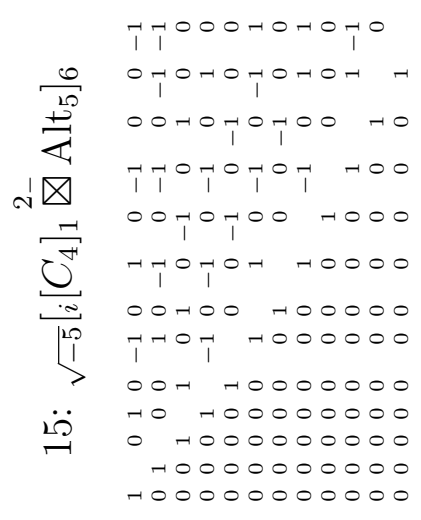

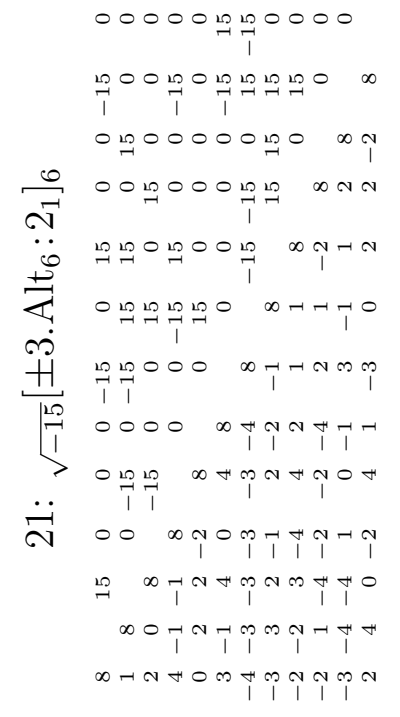
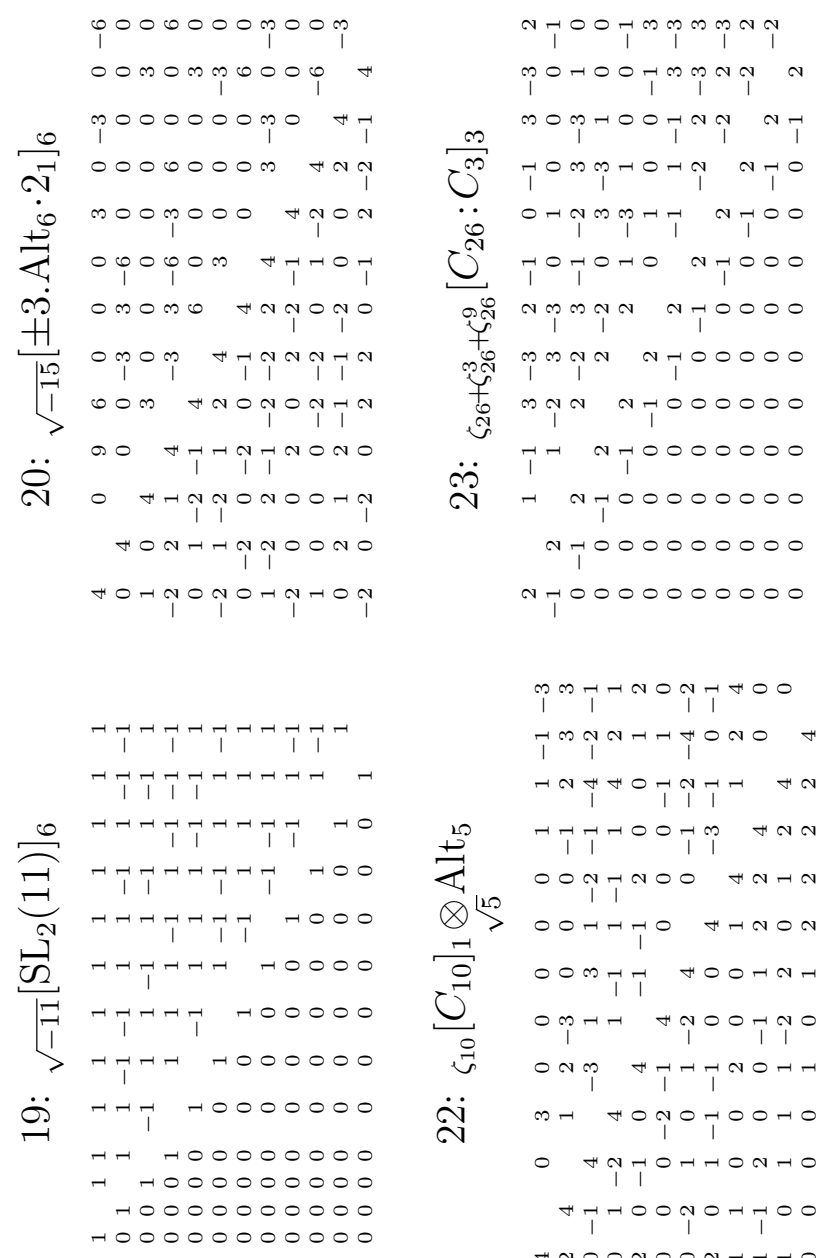


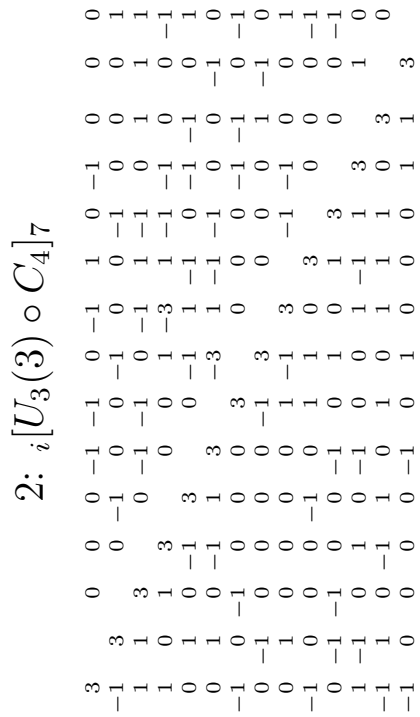

$00400004 \pi-100 \pi$

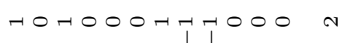

$04 \hat{1}$

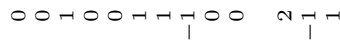

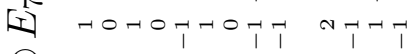

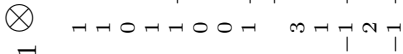

$\tau^{F}-1000000 \mathrm{mH} H \mathrm{H}$

$000000 \mathrm{nH} h \mathrm{H}, \mathrm{H}$

00000 nOHHOOHO

$\pi$
$\cdots$

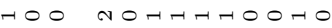

Ho $h \pi t h r h t 00 \pi 0$

o nhohto0, nthotho

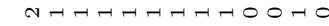

a $0000000 \%$
6

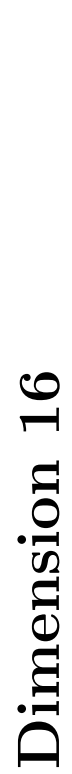

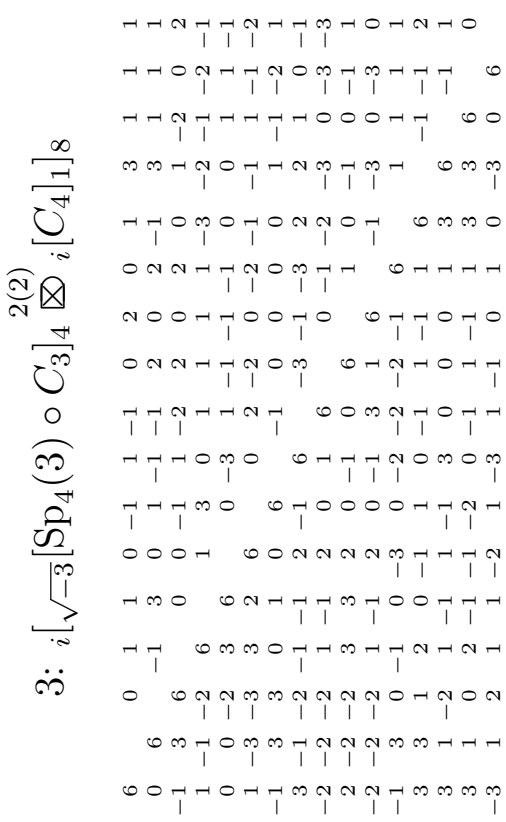

$00^{1}, 0^{1} 0^{1}$

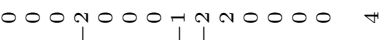
$000 r^{1}, 0^{1}, 0^{4}$ $h 4 h 004000 h 40$ hno

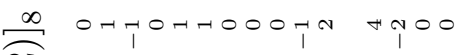

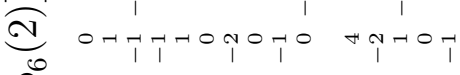

की ।

$\overbrace{4}^{4}$ h000

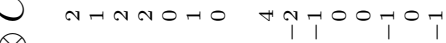

8 ohonoh thonnant

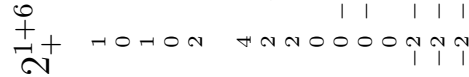

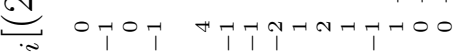

i்

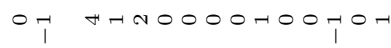

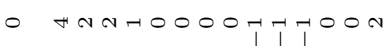

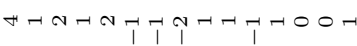

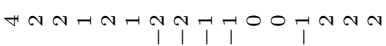



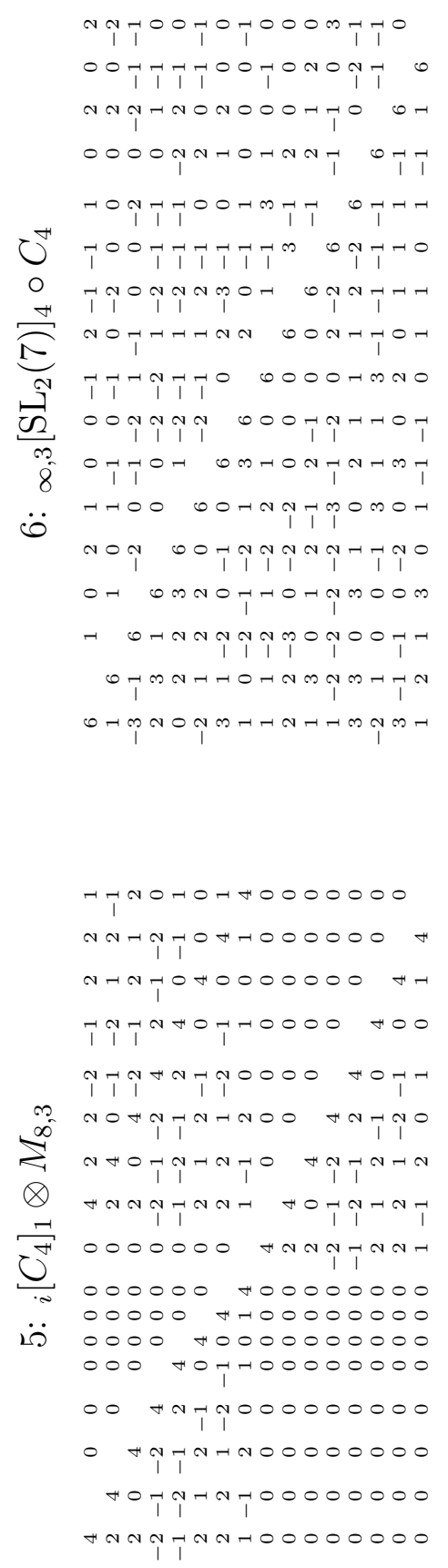
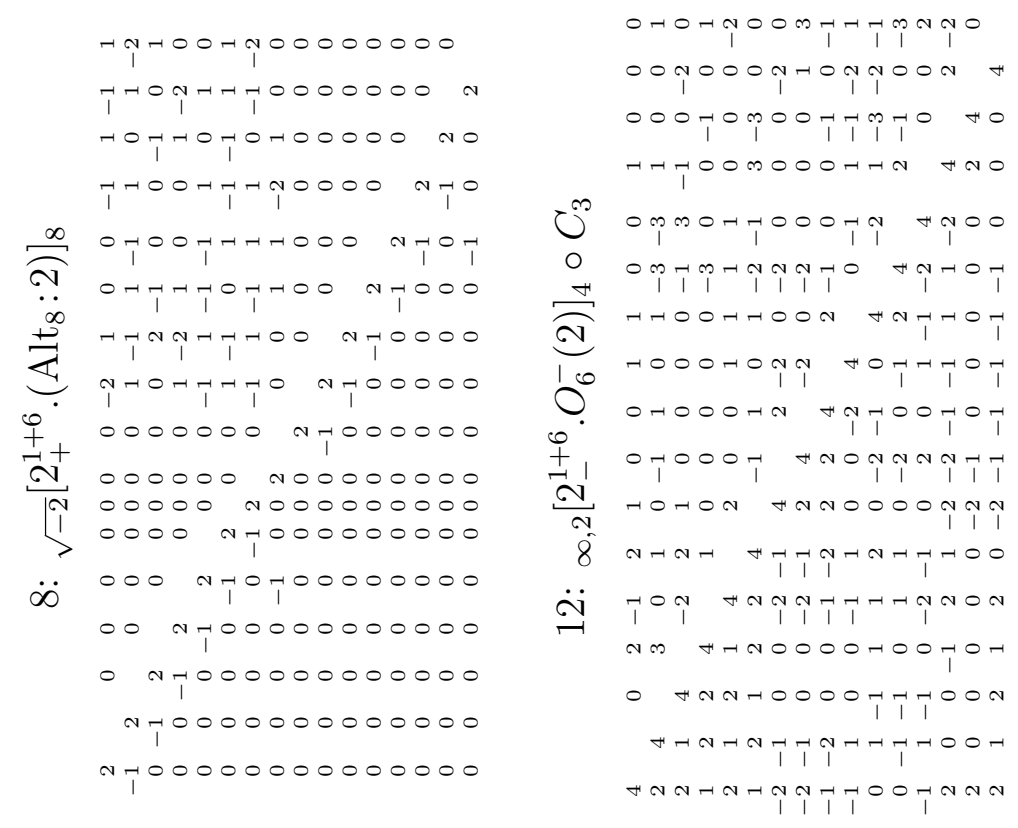

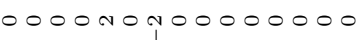

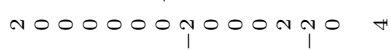
nONODODOOONOO HO oOdnoOJonono hno

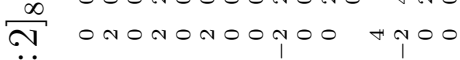
$\underset{\pi}{\pi} 0$ h

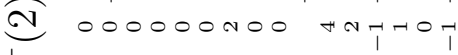
$\int_{100}^{0} 0000000 \mathrm{~h}$ h

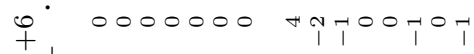
ก 000000 HNOT) d 00000 HnNo000Nin $\stackrel{i n}{1}$

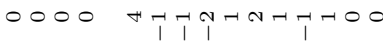

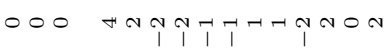
N
$\cdots$

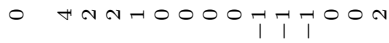

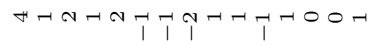

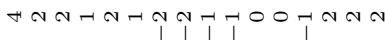

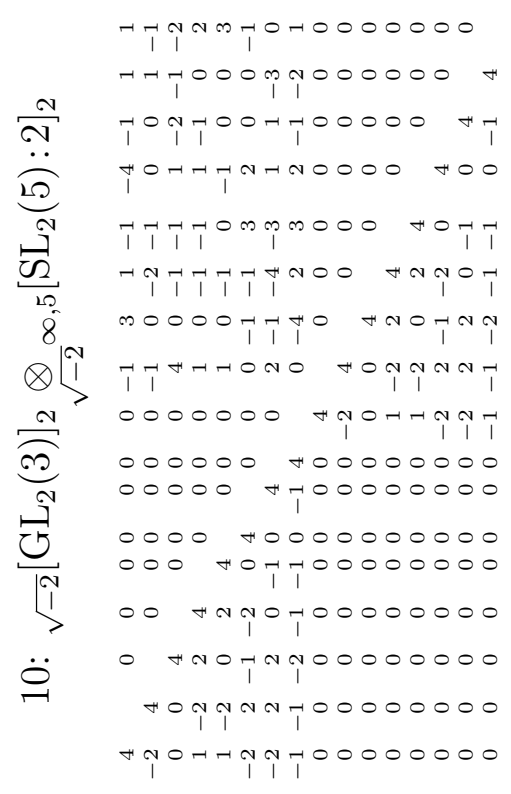



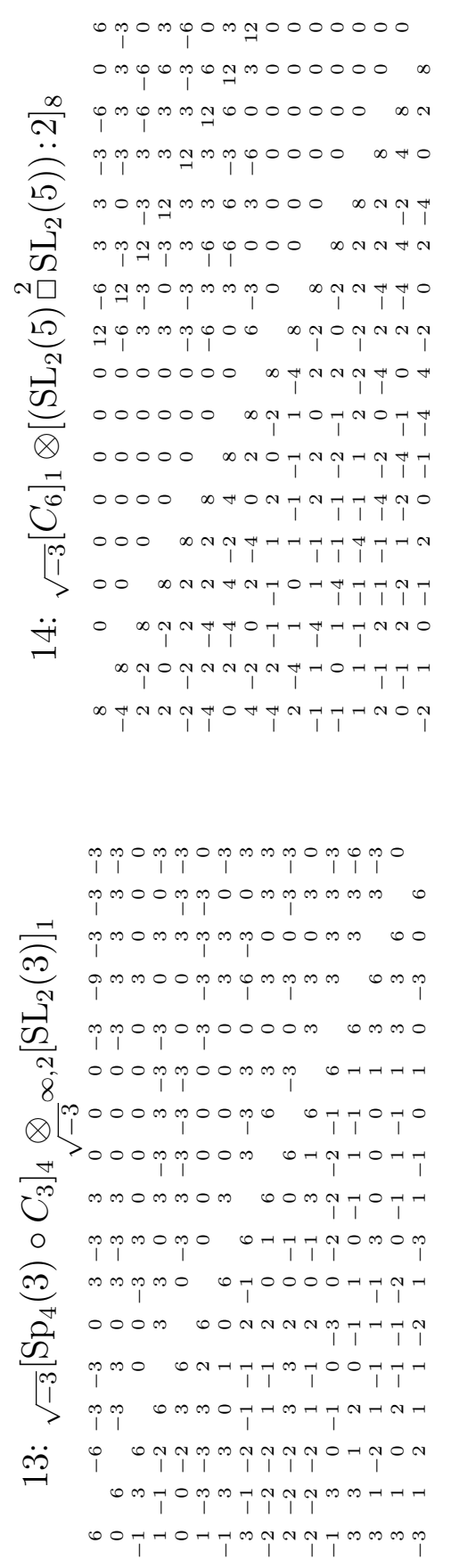

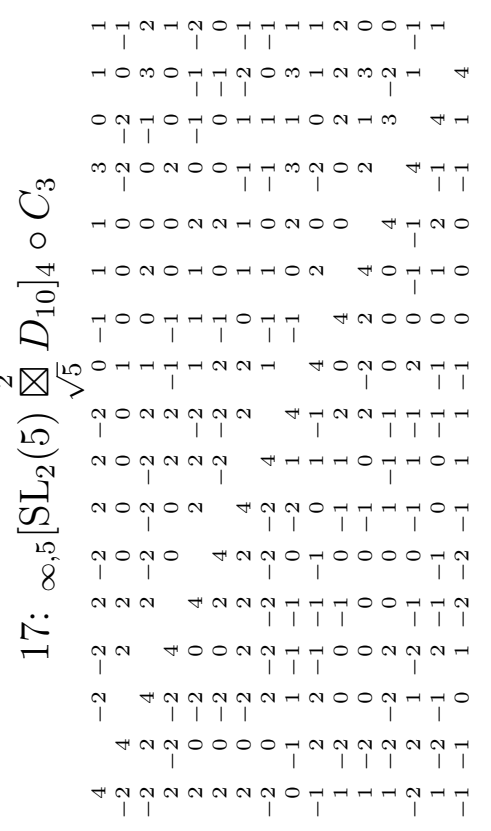

m opopi pmpo

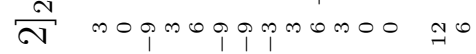
000 p 000000 jom Iito po0pmp p p N N NOPO

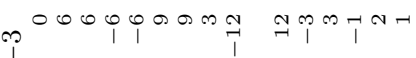
$\theta$ 00000000 TNMOMON

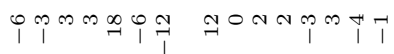

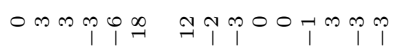

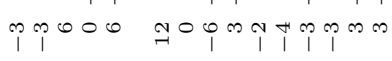

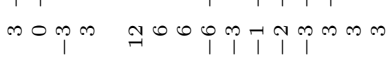

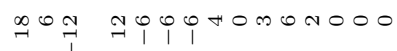
i

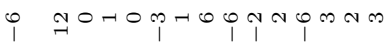

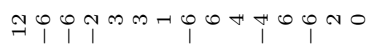
TOM

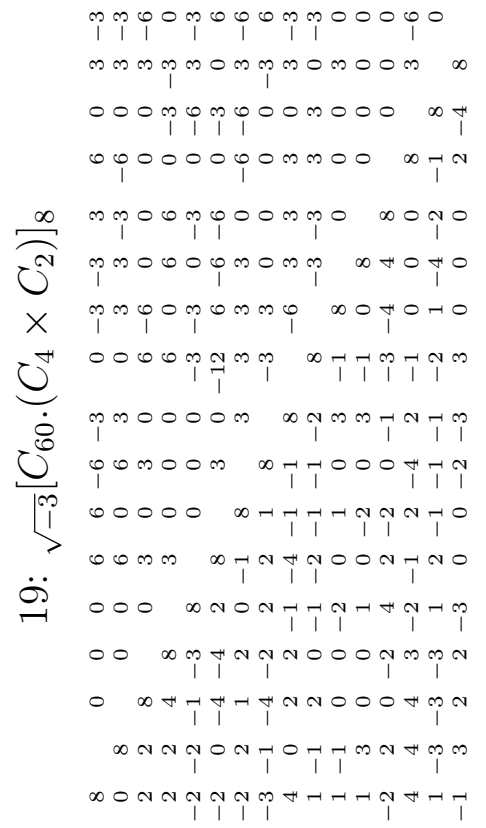

Híli H⿻ind H

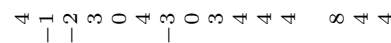

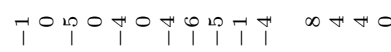

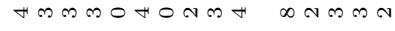

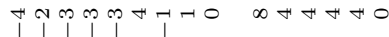

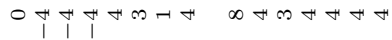

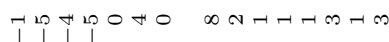

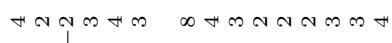
Hop

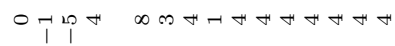

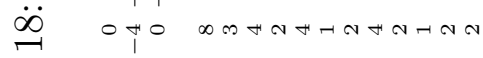
to $\quad$ tratrtrmannm

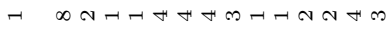
$\infty$ H

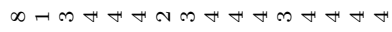



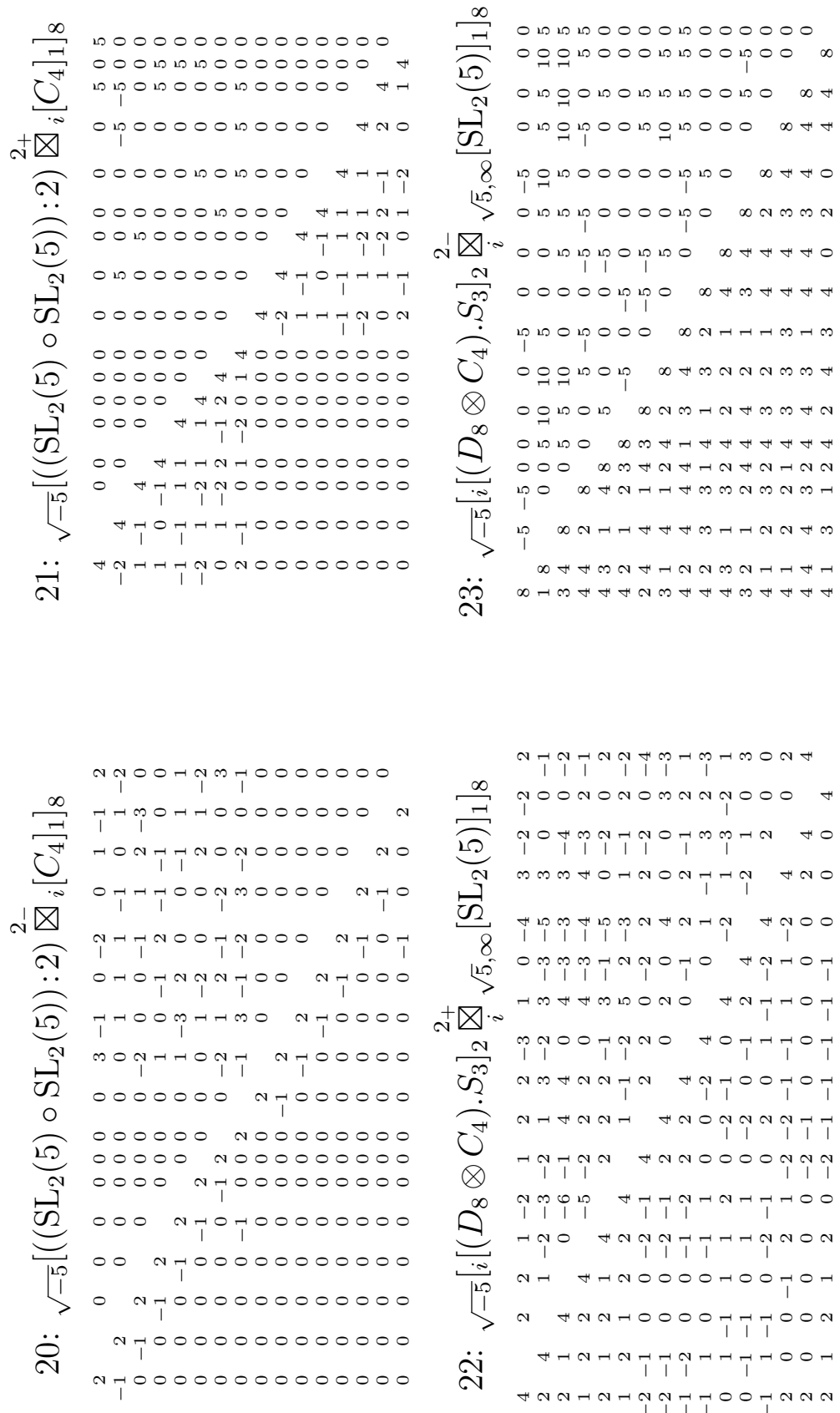

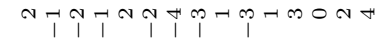

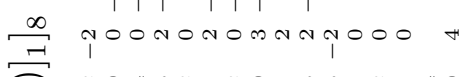

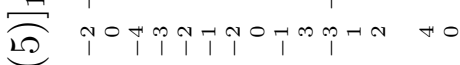
N mmathonHA HNo $\vec{\sim}$ 8 lip

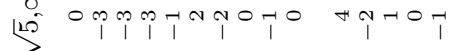

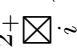
rn $\rightarrow$ anono natanta thto

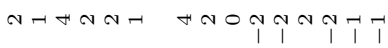

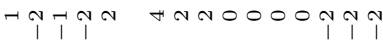
$\otimes_{\infty}$ nmol

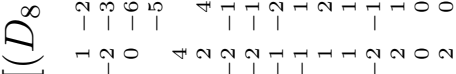
$\frac{1}{20}$

nh Hanoopohoohoh

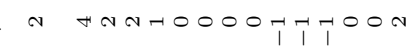
$\ddot{ஸ}$

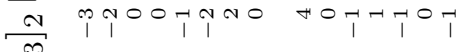

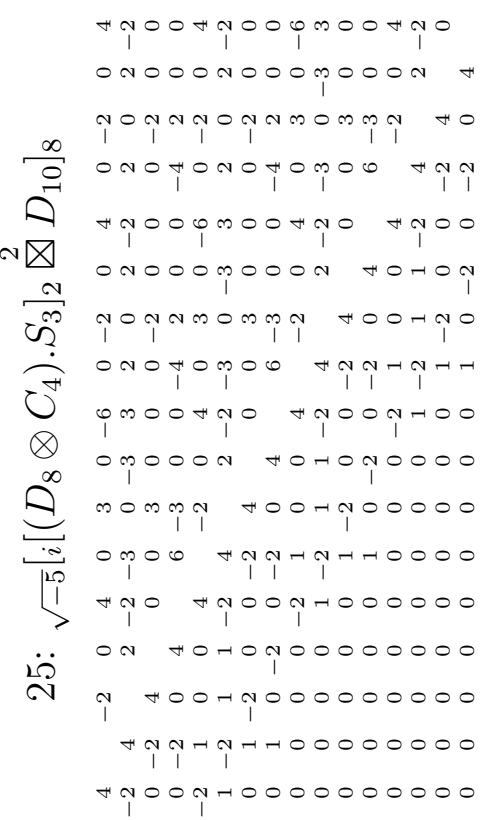

hnm NOMHH, HOH NONOM H $000004 t$ mo $0004 \pi$ H

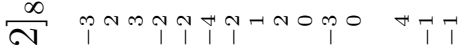
莙

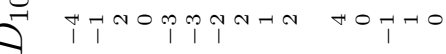

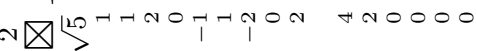
(2)

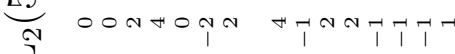

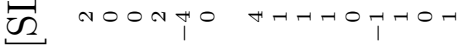
$\delta_{0}$

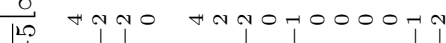

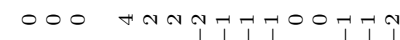

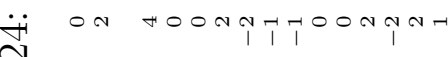

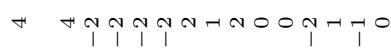
HaTO AT) 

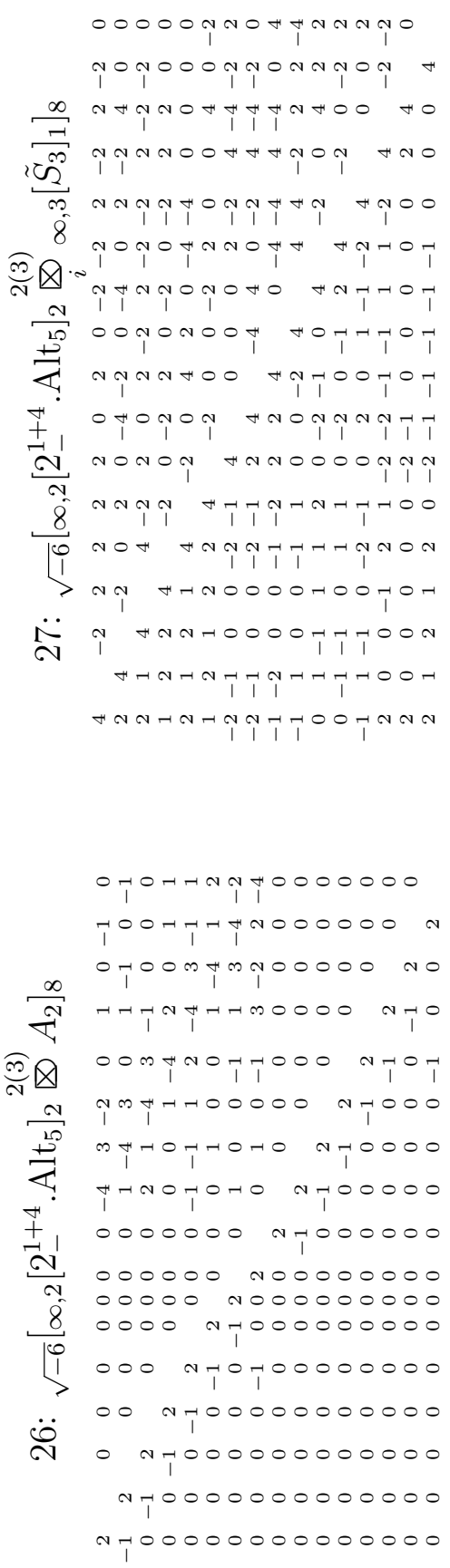

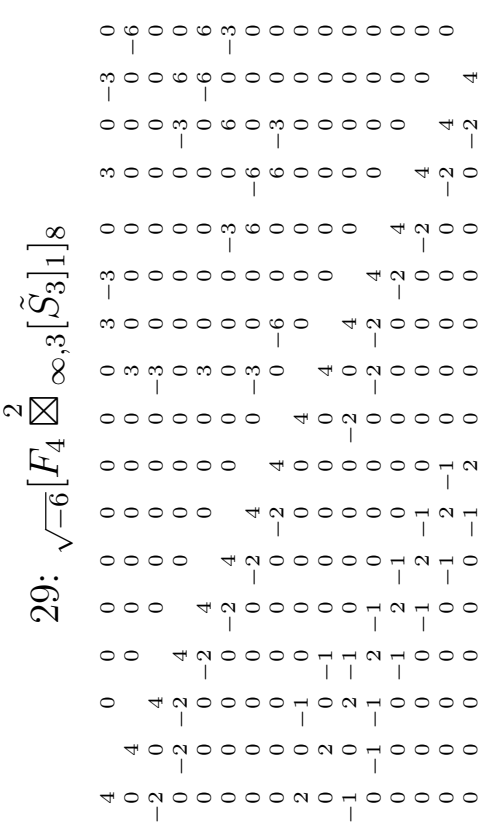

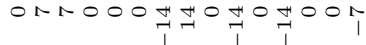 OOOONRNTOONOKN A

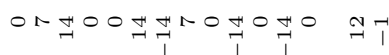

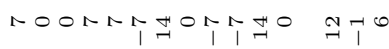 $\frac{\pi}{n}$

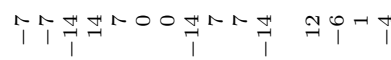

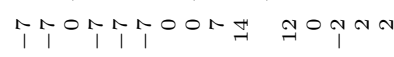

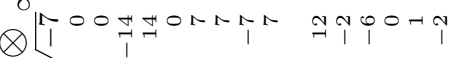 Poonoookn a jhanha 几个O

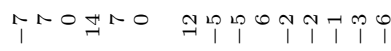 nonor hohthonhth O

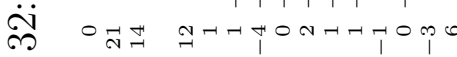

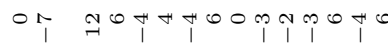 几

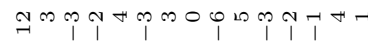

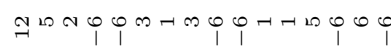

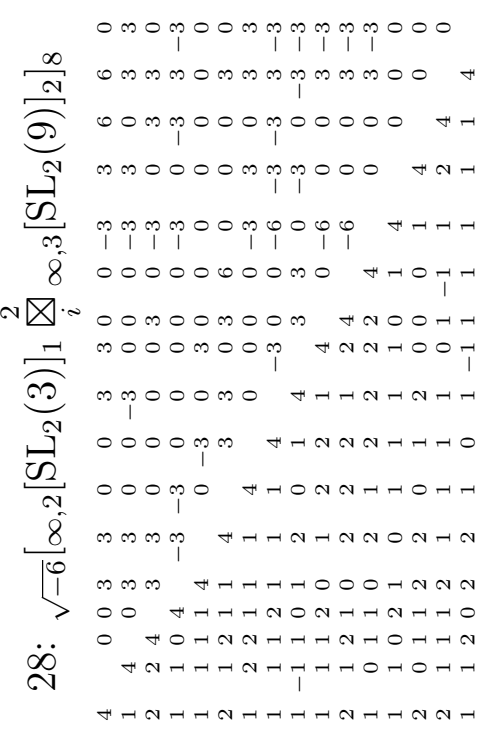

000000090000000 10000000000000 H 0000000000000 H i 000000000000 H00 $\frac{\pi}{N} 00000000000$ HNO $\underset{\pi}{\pi} 00000000000$ Hhithith $\hat{T} 000000000$ Hhi Q 00000000 HAT H I 0000000 Hin 10000000 H00000000 100000 H h 100000000 0000 H0000000000 $\ddot{\dot{0}} 000$ h h0000000000

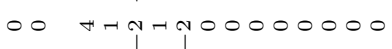

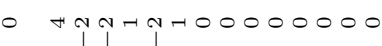
$4 h 4000000000000$ $H A H T-1,000000000000$ 

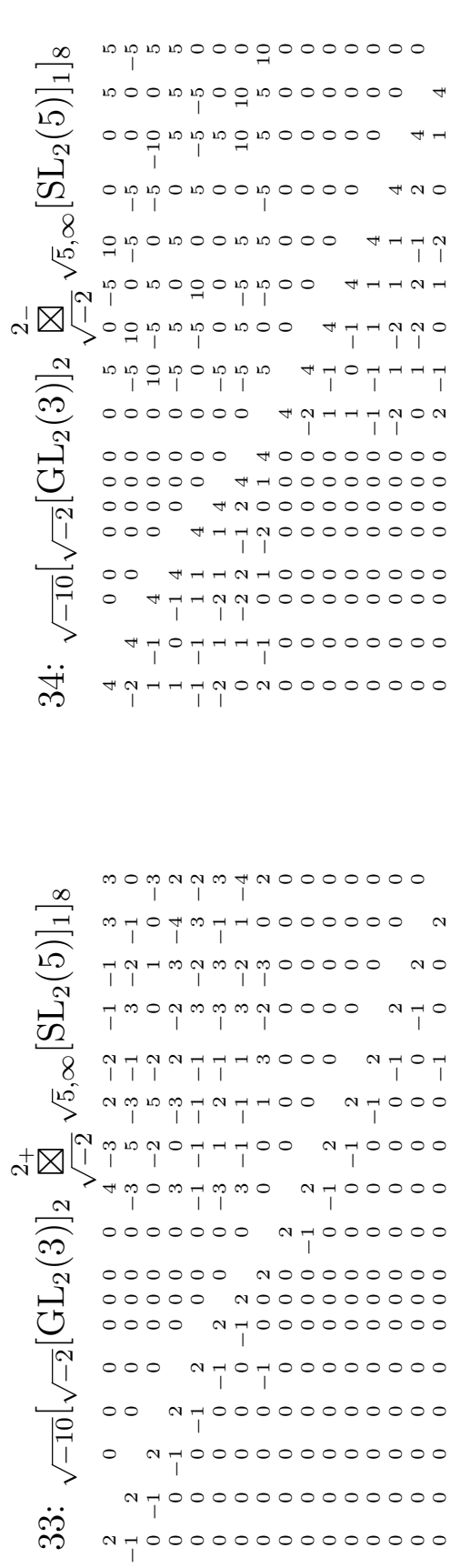
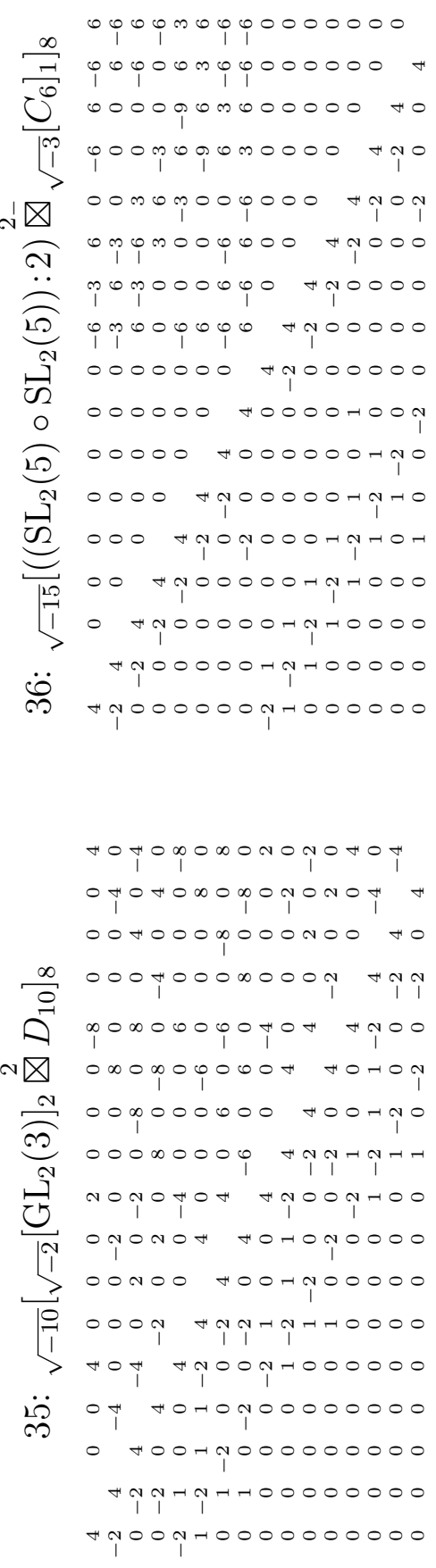

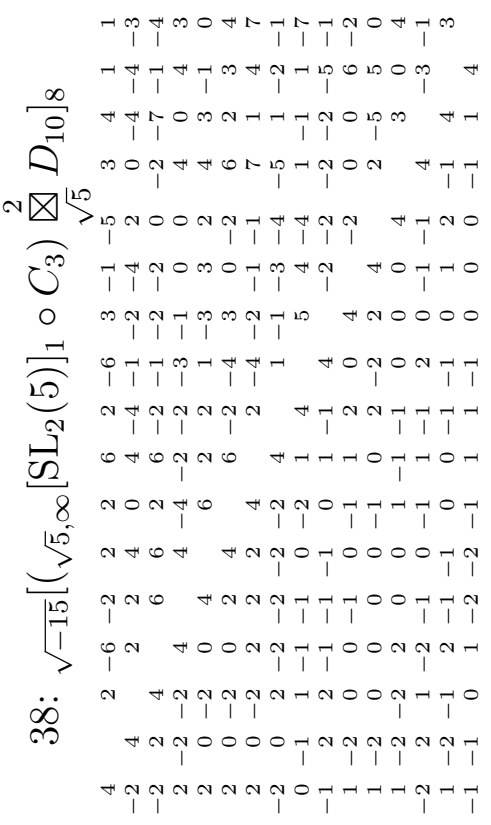

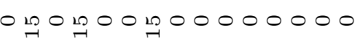
0 学0 $\frac{\sqrt{-\infty}}{2^{\infty}}$ त田

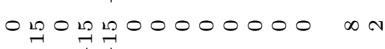

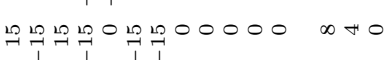

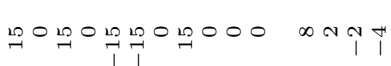
ชิ

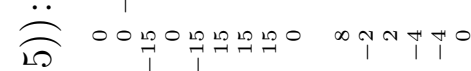
ก $0000000 \pi^{1}$

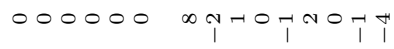
00000 m nOH

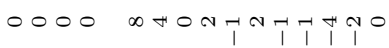

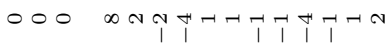

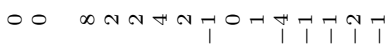
$0 \quad \infty \pi)^{i}$ m $\infty \operatorname{li}^{1}, \frac{1}{1}$ 


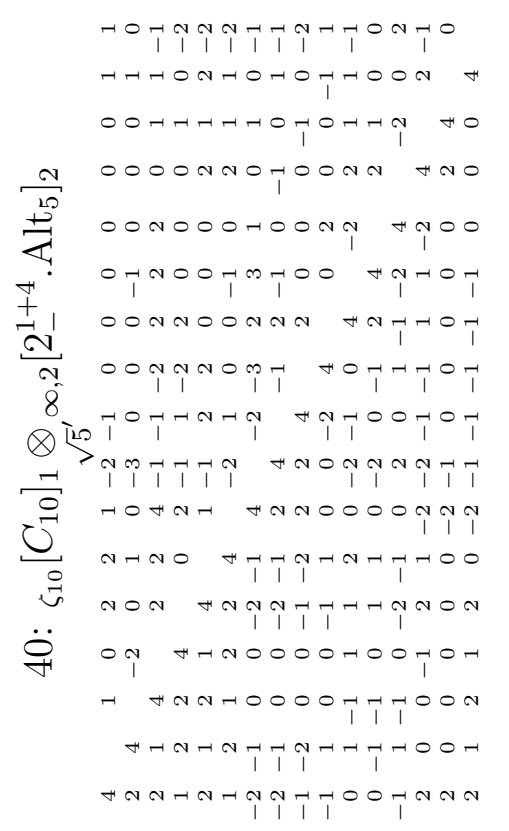

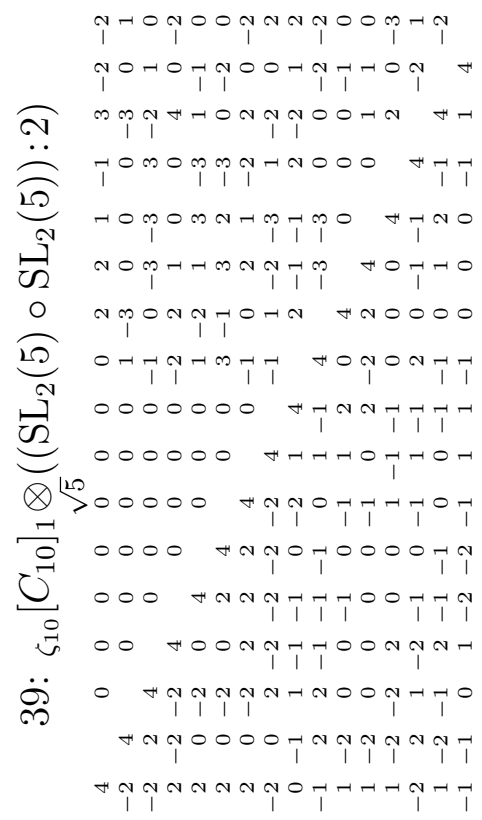
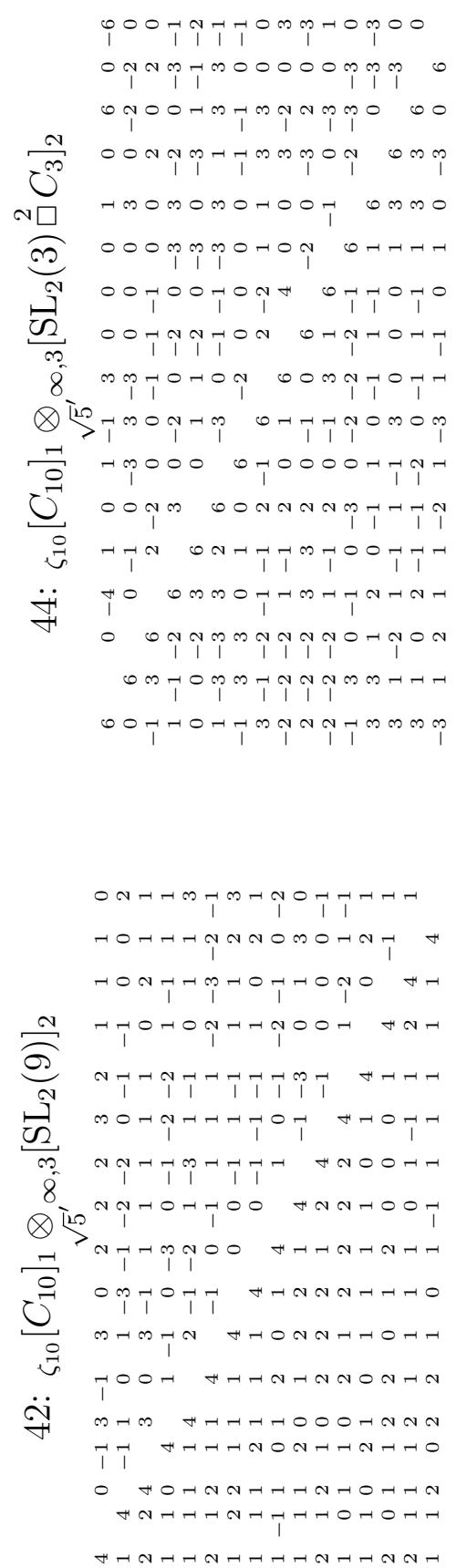

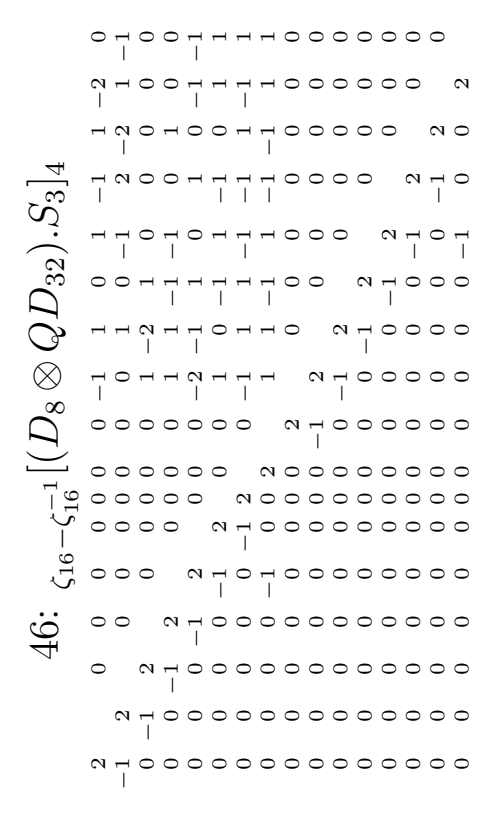

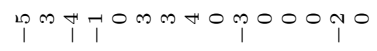

$$
\begin{aligned}
& \text { i }
\end{aligned}
$$

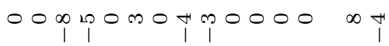

$$
\begin{aligned}
& \text { OOOnOp poon }
\end{aligned}
$$

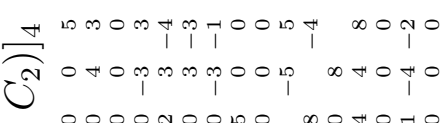

$$
\begin{aligned}
& \text { K } 0000 \text { hoomo nohoro }
\end{aligned}
$$

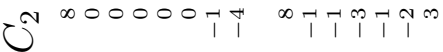

$$
\begin{aligned}
& \text { Hotnm }
\end{aligned}
$$

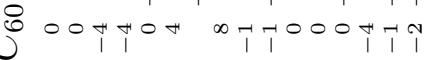

$$
\begin{aligned}
& \underset{-1}{-1} \\
& \text { HOO }
\end{aligned}
$$

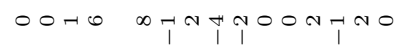

$$
\begin{aligned}
& \ddot{r} \\
& \text { me manthithra }
\end{aligned}
$$

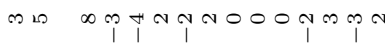

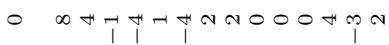

$$
\begin{aligned}
& \text { mani n n }
\end{aligned}
$$

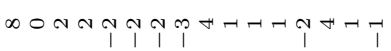



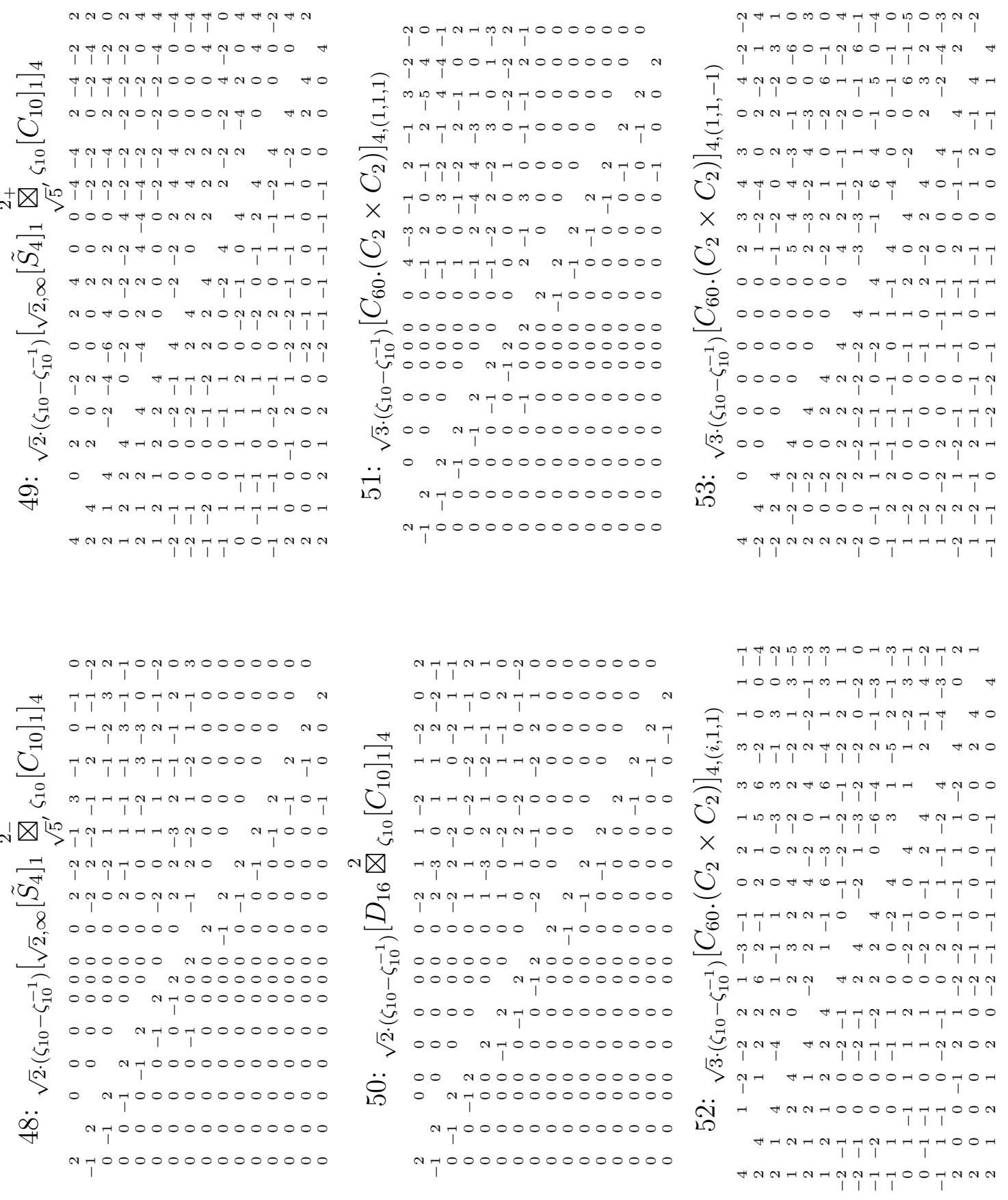


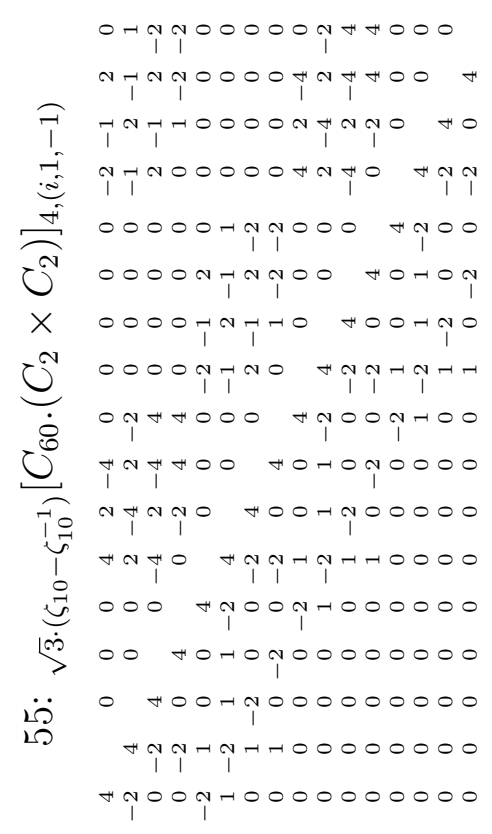

pnno0p po00000000 0 p n m p p momponmonoor to

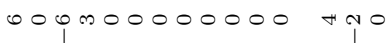
Op p p p n pip momnoO

U

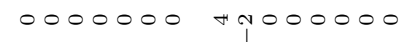
000000 h0000700 H 00000 H000000hi 0000 hijo, o,

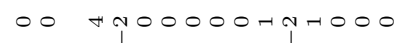

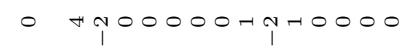

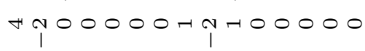
hino

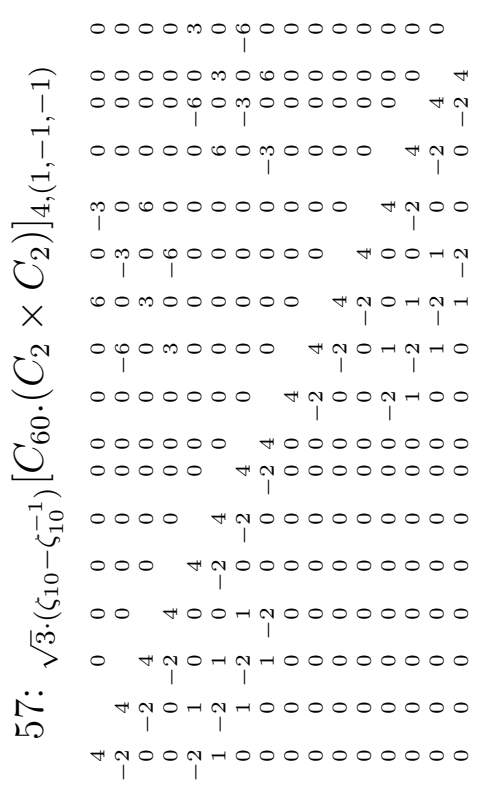

mm nOO OOOnnmoOppinom I $0000 m p m p i p p 0000$ nno nom nomp on OOp00000000 0n0 o00000000m ohthr Doomolom O00m00m $0 \pi$ I mpoom o Omp p o omp li 000 o, on ontr o o m nothmhthrnh - ONNMnNNNNOHAN on DO H H

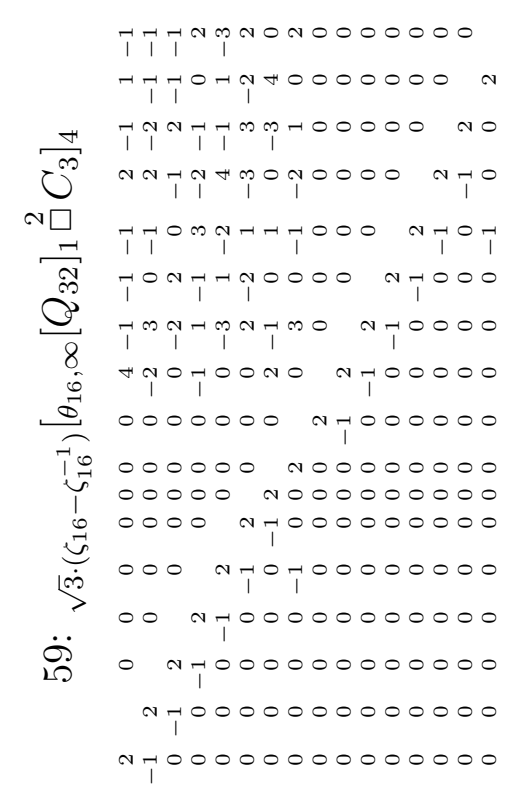

poOnm o p p poompopopon ᄀ $000000000 m_{1} 0 N 0 \infty H$

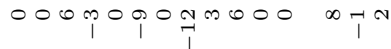
ipompipipmo non

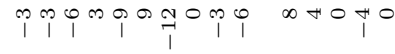
pnOOMOMUO nOHOHO ○一 nం o o poom nhtoo hit

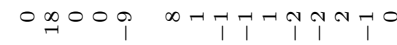
OOML

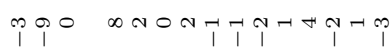

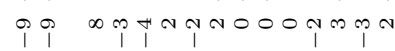
$\dot{\infty}$
$\dot{0}$

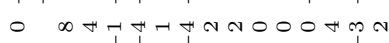
$\infty$ n n n mONNAT) 

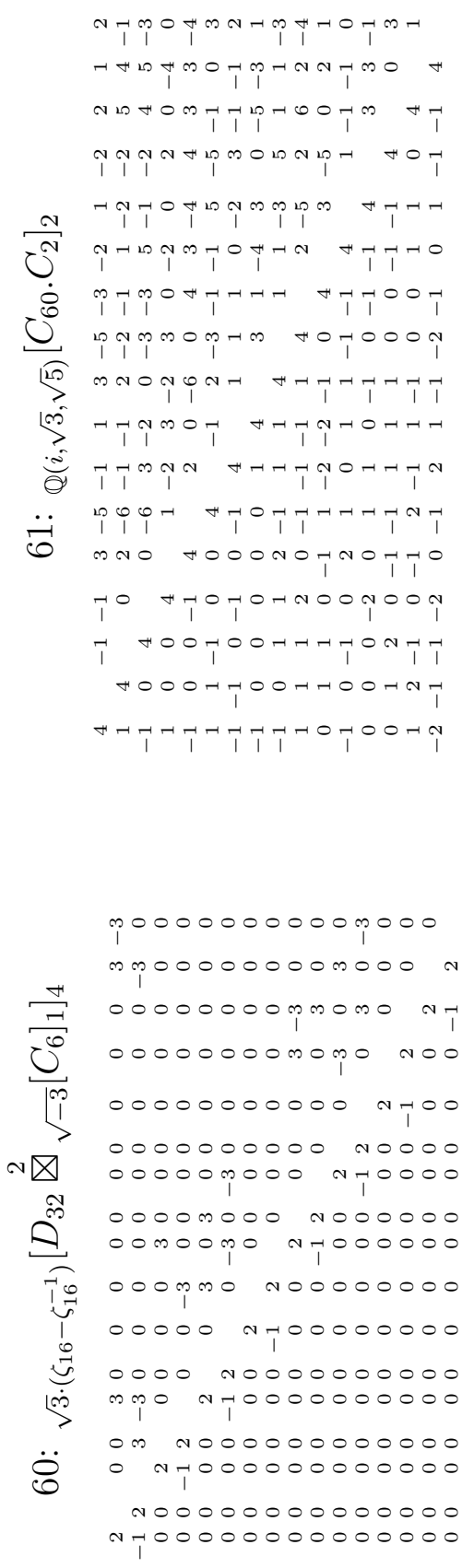

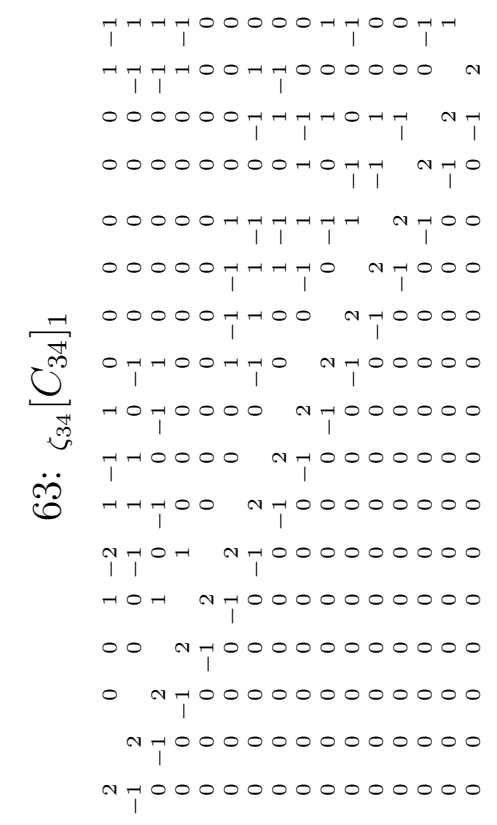

400000000000007 $00000000000004-1$ $000000000000 \pi-10$

$\mathrm{H} \quad 000000000004-100$

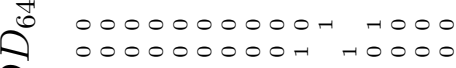
S 000000007 H

- $0000000 \pi r 000000$

I $000000 \pi-10000000$

h $000007-100000000$

ले $00007-1000000000$

ii $00 \pi r 00000000000$

$\ddot{\mathrm{i}} 0-r 000000000000$ $r-r 0000000000000$ $-100000000000000$ 1000000000000000 


\section{A.9 Dimension 18}

1: ${ }_{i}\left[C_{4}\right]_{1} \otimes A_{9}$

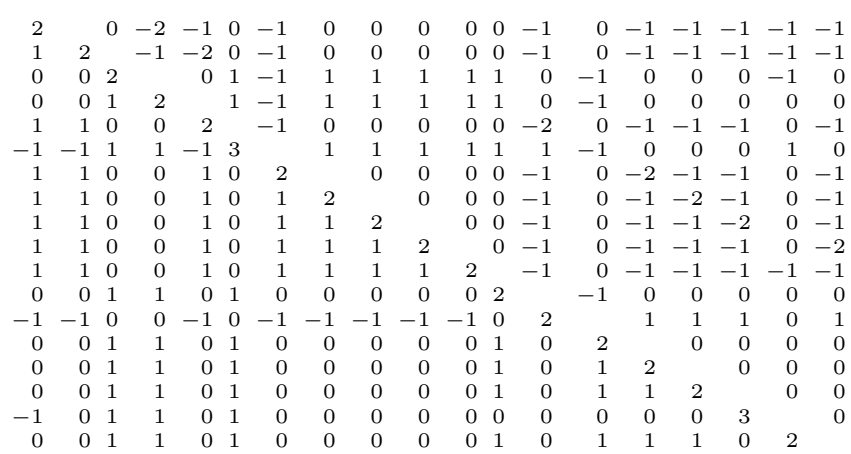

2: $\sqrt{-3}\left[ \pm 3_{+}^{1+4}: \mathrm{Sp}_{4}(3)\right]_{9}$

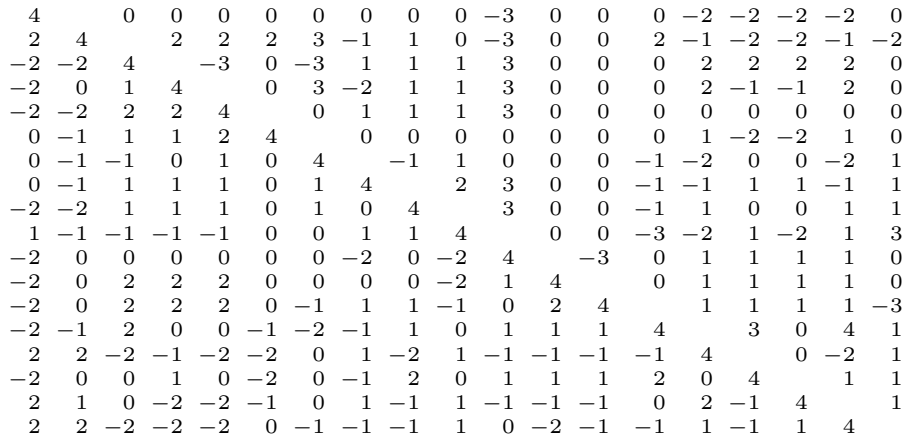

4: $\sqrt{-3}\left[ \pm 3 . M_{10}\right]_{9}$

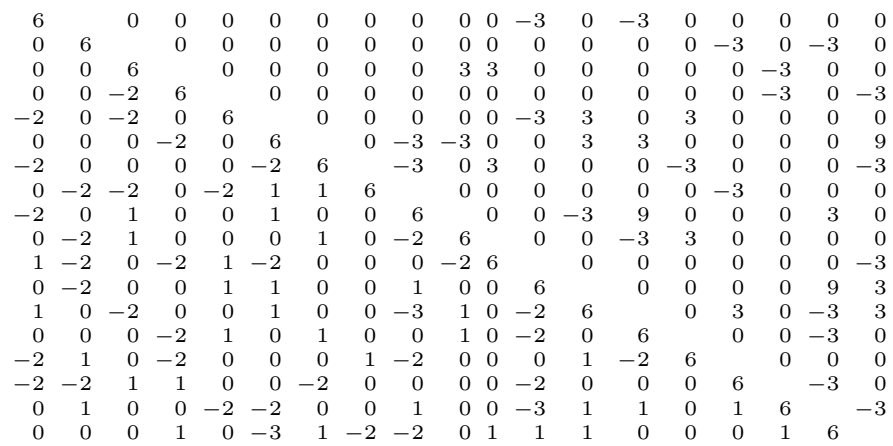

$$
\text { 5: } \sqrt{-7}\left[ \pm\left(\mathrm{L}_{2}(7) \underset{\sqrt{-7}}{\otimes} \mathrm{L}_{2}(7)\right): 2\right]_{9}
$$

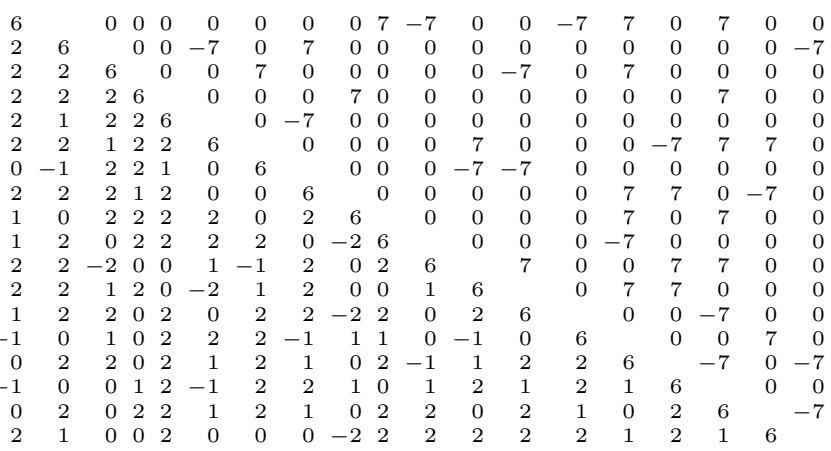




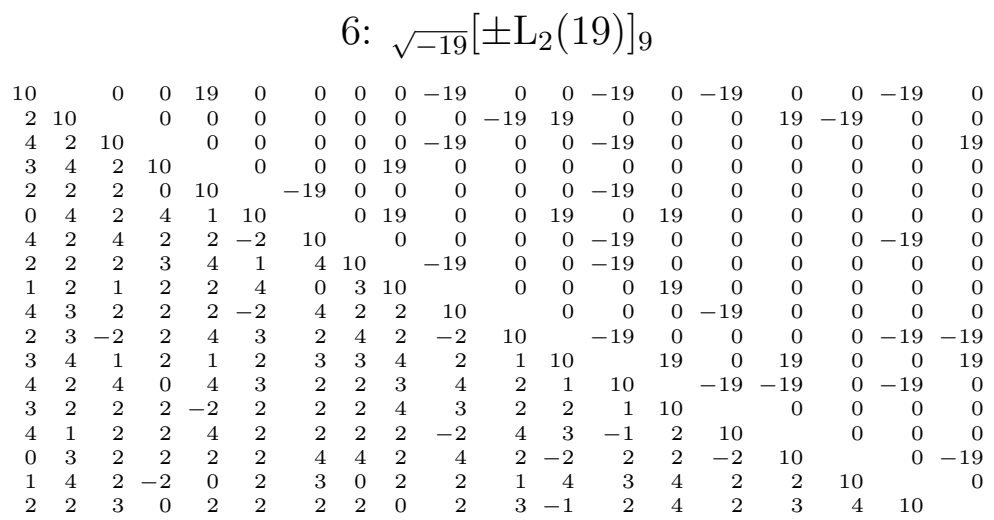

\section{A.10 Dimension 20}

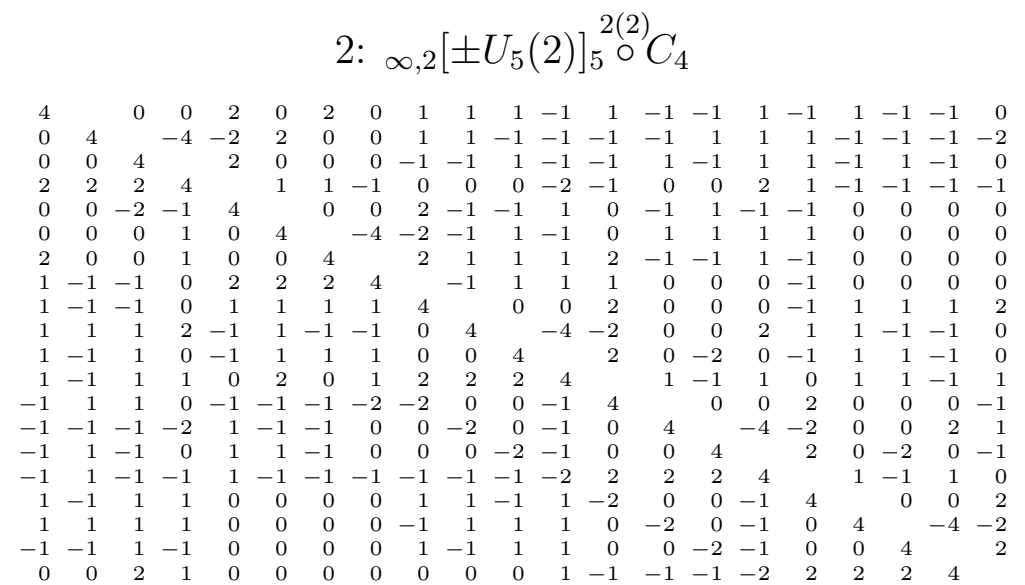

3: ${ }_{i}\left[C_{4}\right]_{1} \otimes\left[\left(C_{6} \times S_{4}(3)\right) \cdot 2\right]_{10}$

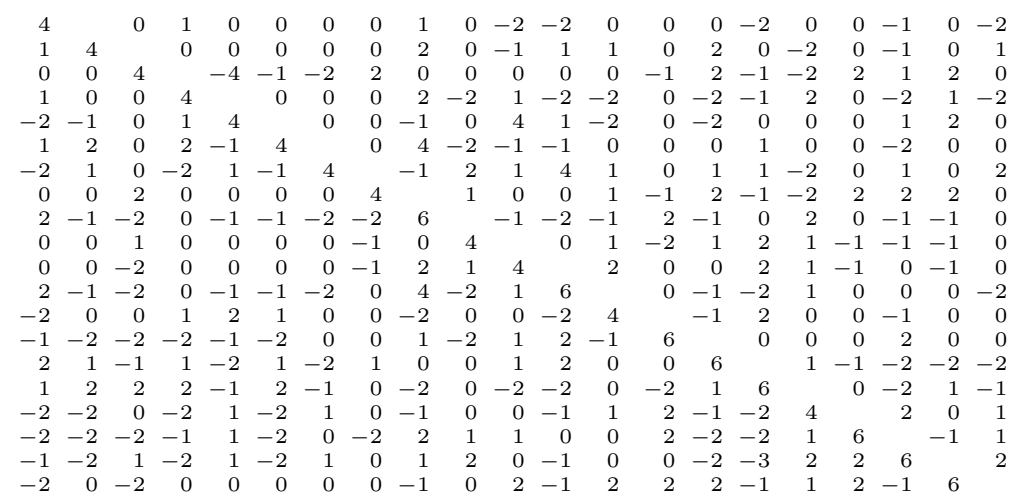


4: ${ }_{i}\left[\left(D_{8} \otimes C_{4}\right) \cdot S_{3}\right]_{2} \otimes A_{5}$

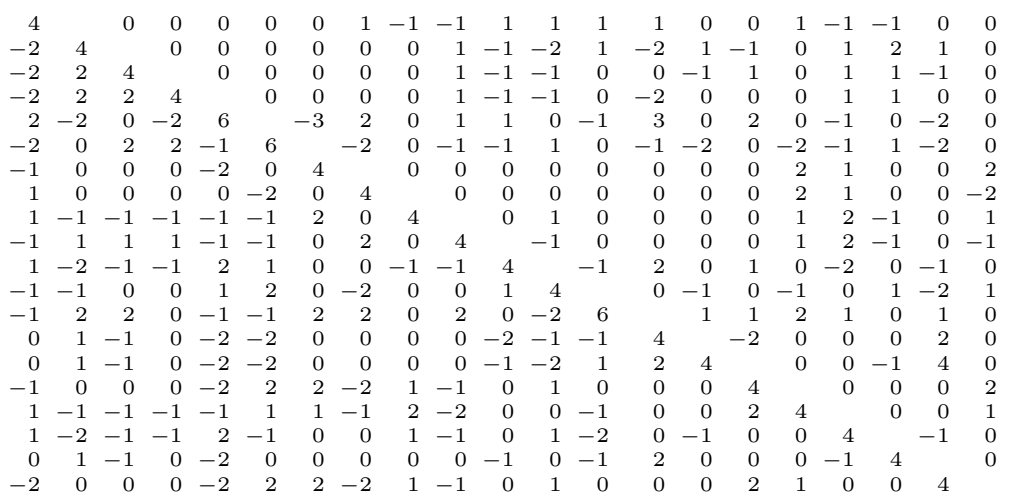

5: ${ }_{i}\left[\left[ \pm S_{6}\right]_{10} \stackrel{2(2)}{\otimes}{ }_{i}\left[C_{4}\right]_{1}\right]_{10}$

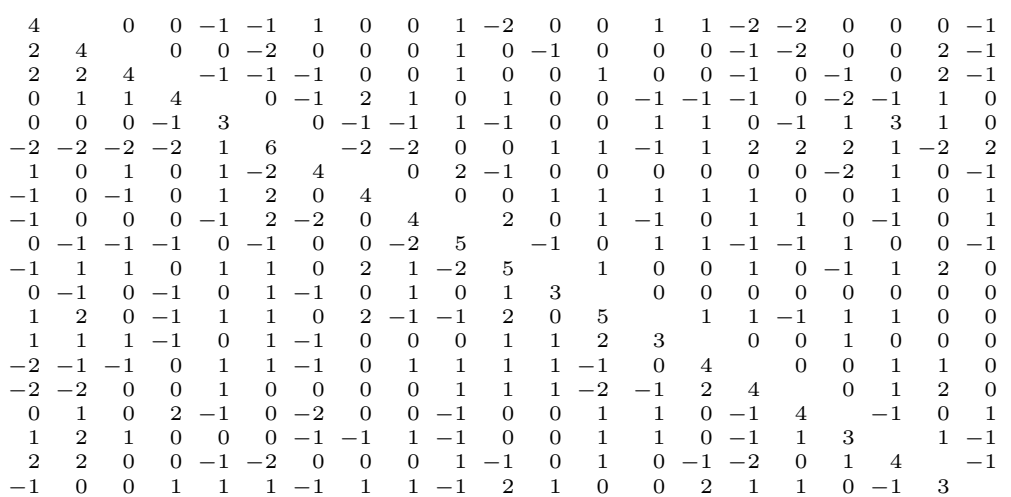

\section{6: ${ }_{i}\left[C_{4}\right]_{1} \otimes A_{10}^{(2)}$}

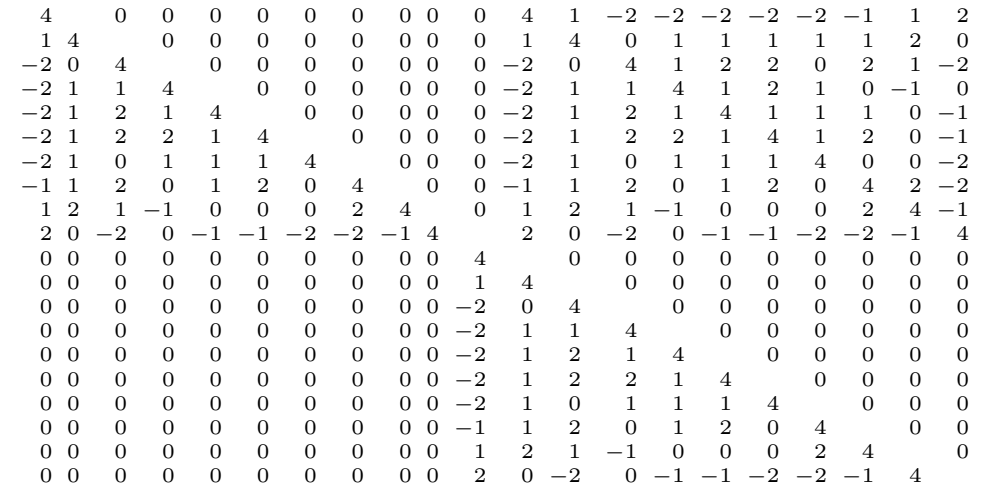

$$
\text { 7: } \infty, 2\left[\mathrm{SL}_{2}(11)\right]_{5} \stackrel{2(2)}{\square} C_{4}
$$

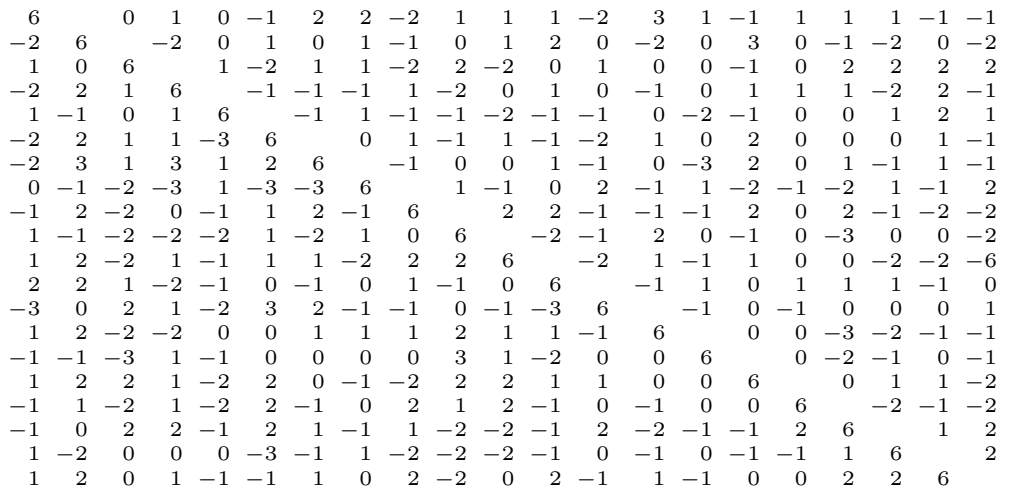


8: ${ }_{i}\left[C_{4}\right]_{1} \otimes A_{10}^{(3)}$

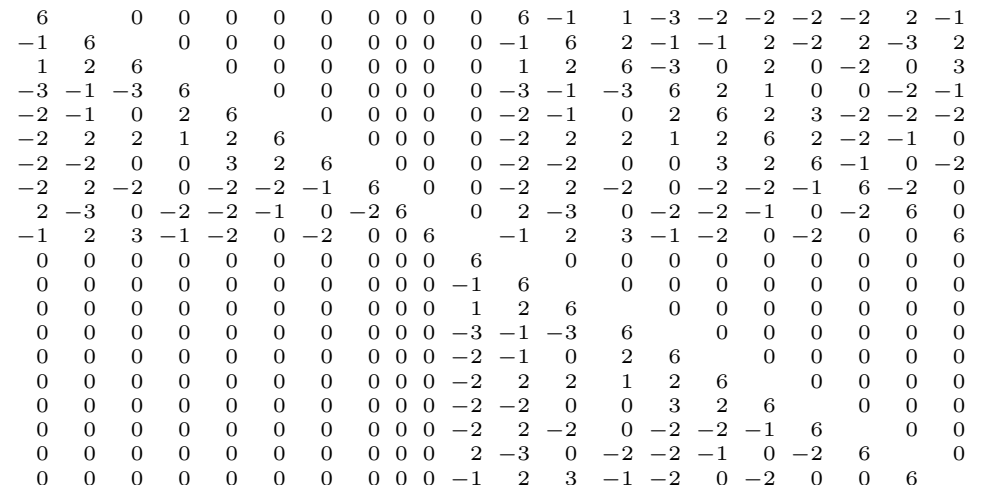

9: $\sqrt{-2}\left[\infty, 2\left[ \pm U_{5}(2)\right]_{5}: 2\right]_{10}$

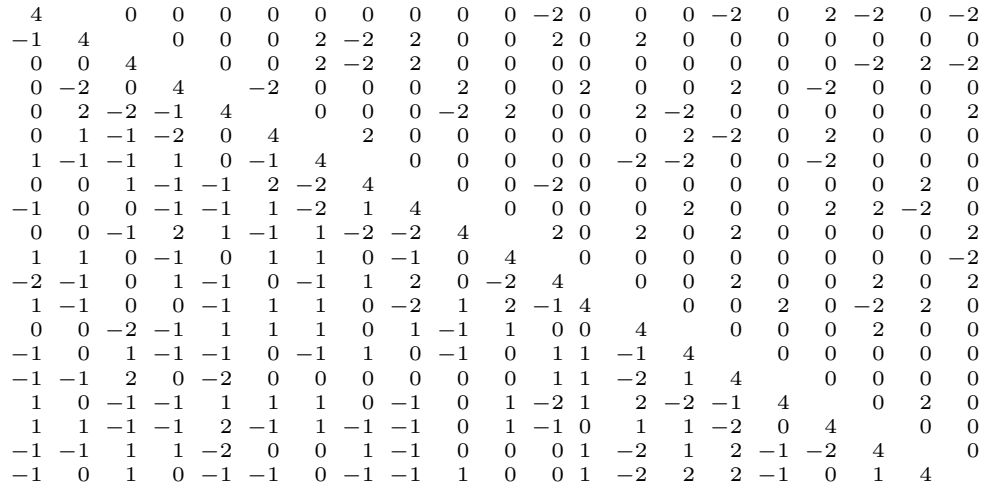

10: $\sqrt{-2}\left[2 . M_{12}: 2\right]_{10}$

$\begin{array}{rrrrrrrrrrrrrrrrrrrrr}4 & & 2 & -2 & 0 & -2 & -2 & 0 & 0 & 0 & 2 & 2 & 0 & 0 & -2 & 0 & -2 & -2 & 2 & 0 & 0 \\ -2 & 4 & & 0 & -2 & 2 & 0 & 0 & 0 & 0 & 0 & 0 & 0 & 0 & 2 & 0 & 2 & 2 & 2 & 0 & 2 \\ 1 & -1 & 4 & & 2 & 0 & 0 & 0 & 0 & 0 & 0 & 0 & 0 & 0 & -2 & 0 & -2 & -2 & 0 & 0 & 0 \\ 0 & 0 & 0 & 4 & & -2 & 0 & -2 & -2 & -2 & 0 & 0 & 2 & 0 & 0 & 2 & 0 & 0 & 0 & 0 & 0 \\ 1 & -2 & 2 & -1 & 4 & & -2 & 0 & 0 & 0 & 0 & -2 & 0 & 2 & 0 & 0 & -2 & 0 & 0 & 0 & 0 \\ 1 & -1 & 0 & 0 & 0 & 4 & & 0 & 0 & 2 & 0 & 0 & -2 & 2 & 0 & -2 & 0 & -2 & 0 & 2 & -2 \\ 0 & 0 & 2 & -1 & 2 & -1 & 4 & & 0 & 0 & 0 & 0 & 0 & 0 & -2 & 0 & -2 & 0 & 0 & 0 & 0 \\ -1 & 0 & 2 & 0 & 1 & -1 & 1 & 4 & & -2 & 0 & 0 & 2 & 0 & 0 & 0 & 0 & 0 & 0 & -2 & 0 \\ 0 & -1 & 2 & 0 & 2 & 0 & 1 & 2 & 4 & & 0 & -2 & 2 & 0 & 0 & 2 & 0 & 0 & 0 & 0 & 0 \\ -2 & 2 & 0 & 1 & -1 & -2 & 0 & 1 & 1 & 4 & & -2 & 2 & -2 & 2 & 2 & 2 & 2 & 0 & 0 & 2 \\ -1 & 2 & 0 & 1 & -1 & 0 & 0 & 0 & -1 & 1 & 4 & & 0 & 0 & 2 & 0 & 2 & 0 & 0 & 0 & 2 \\ -2 & 2 & -2 & 1 & -2 & -1 & 0 & -1 & -2 & 1 & 0 & 4 & & 0 & 0 & 0 & 0 & 2 & 0 & 0 & 0 \\ 2 & -2 & 1 & 0 & 2 & -1 & 0 & 0 & 0 & -1 & 0 & -2 & 4 & & 0 & 0 & 0 & 0 & 0 & 0 & 2 \\ -1 & -1 & -1 & -1 & 1 & -1 & 0 & -1 & 0 & 0 & -2 & 1 & 0 & 4 & & 0 & -2 & 2 & -2 & 0 & 0 \\ -1 & 0 & -2 & 0 & -1 & 0 & -1 & -1 & -1 & -1 & -1 & 2 & -1 & 1 & 4 & & 0 & 0 & 0 & 0 & -2 \\ -2 & 0 & 0 & -1 & 0 & 0 & -1 & 1 & 1 & 1 & -1 & 0 & -1 & 2 & 0 & 4 & & 2 & -2 & 0 & 0 \\ -1 & 0 & 0 & -1 & 0 & -2 & 1 & 0 & -1 & 0 & -1 & 1 & 0 & 2 & 0 & 1 & 4 & & -2 & -2 & 0 \\ -1 & 1 & -1 & -1 & 0 & -2 & 0 & 1 & -1 & 1 & 1 & 0 & 1 & 0 & -1 & 0 & 1 & 4 & & -2 & 2 \\ 0 & 1 & 1 & 0 & 0 & -1 & 0 & 1 & 1 & 1 & 0 & -1 & 0 & 0 & -2 & 1 & 1 & 1 & 4 & & 2 \\ -1 & 0 & 1 & -2 & 1 & -2 & 2 & 1 & 1 & 1 & -1 & 0 & 0 & 1 & 0 & 1 & 2 & 1 & 0 & 4 & \end{array}$

11: $\sqrt{-2}\left[\mathrm{GL}_{2}(3)\right]_{2} \otimes A_{5}$

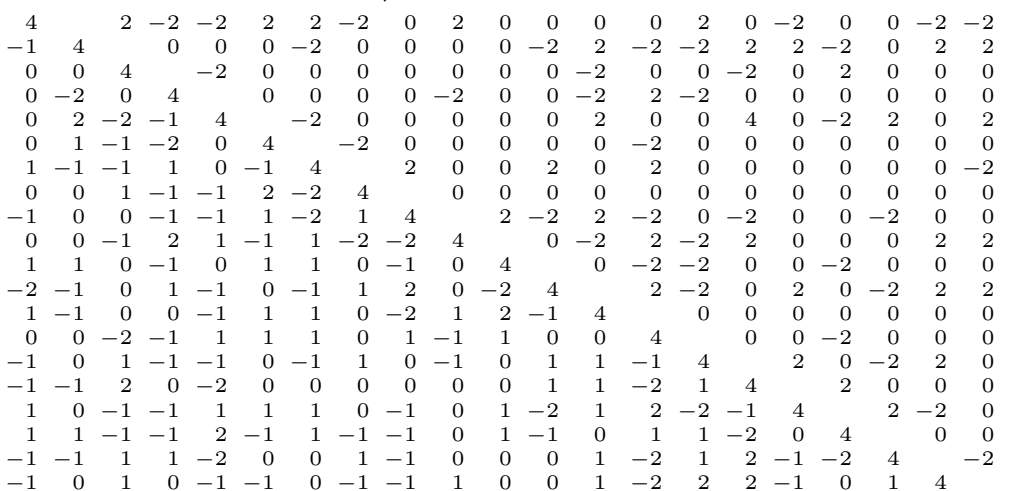


13: $\infty, 2\left[ \pm U_{5}(2)\right]_{5} \circ C_{3}$

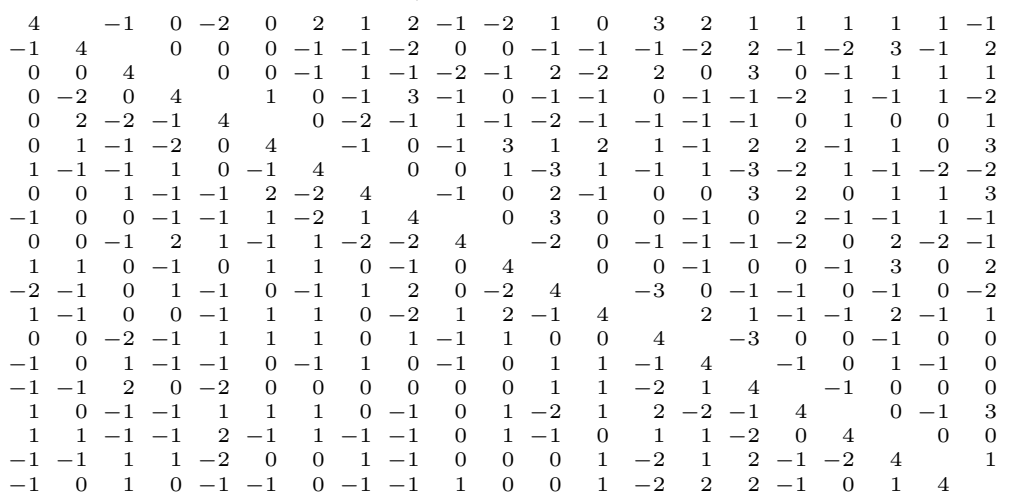

14: $\sqrt{-3}\left[ \pm S_{4}(3) \circ C_{3}\right]_{5} \otimes{ }_{\infty, 2}\left[\mathrm{SL}_{2}(3)\right]_{1}$

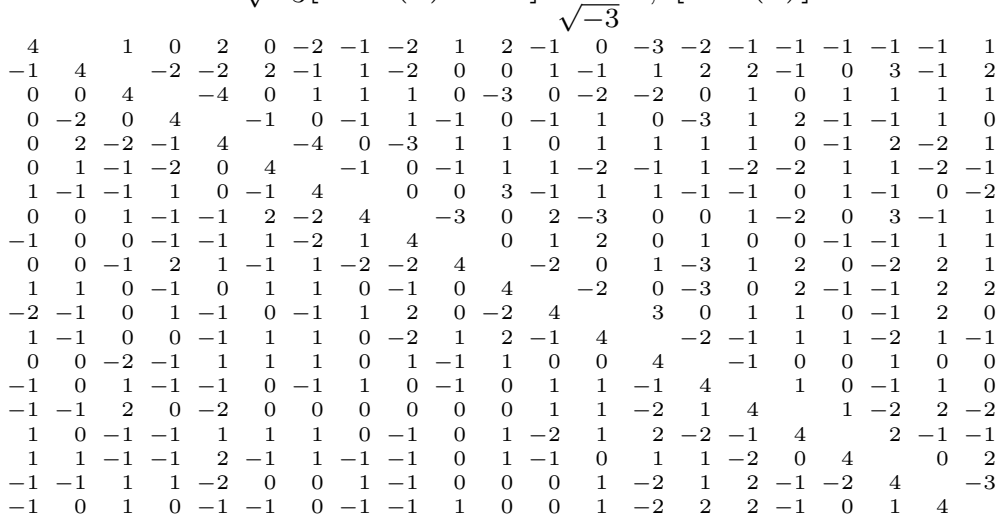

15: $\sqrt{-3}\left[ \pm U_{4}(2) \circ C_{3}\right]_{10}$

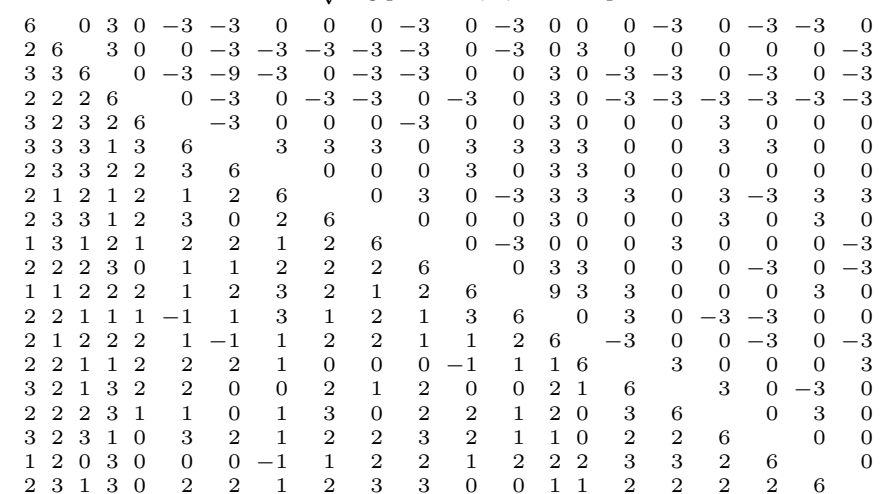

16: $\sqrt{-3}\left[ \pm \mathrm{L}_{2}(11)^{2(3)} \otimes_{\sqrt{-3}}\left[C_{6}\right]_{1}\right]_{10}$

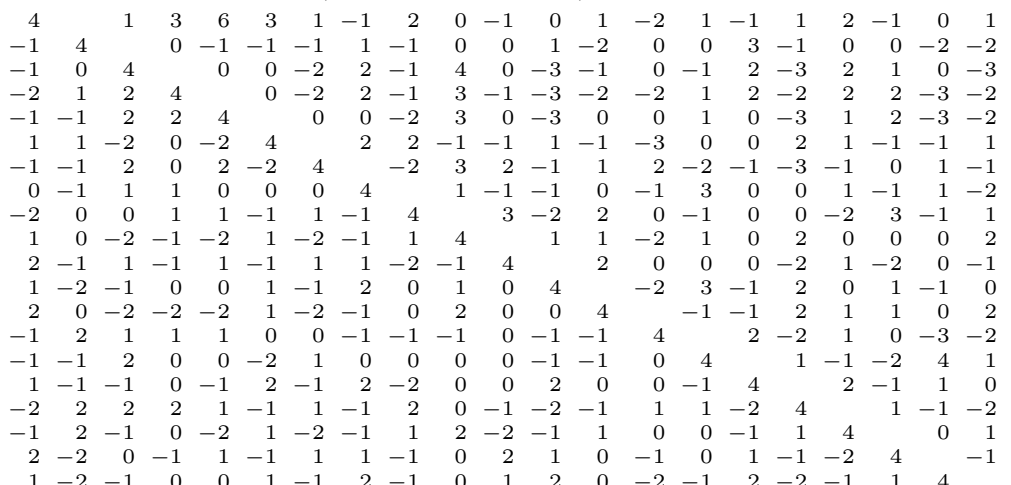




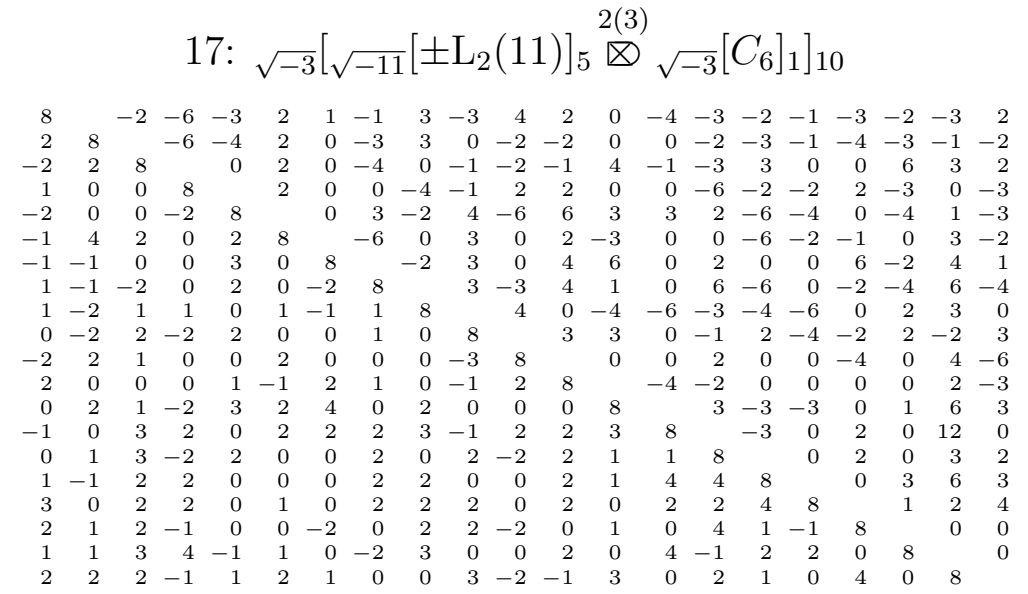

18: $\sqrt{-3}\left[C_{6}\right]_{1} \otimes A_{10}^{(2)}$

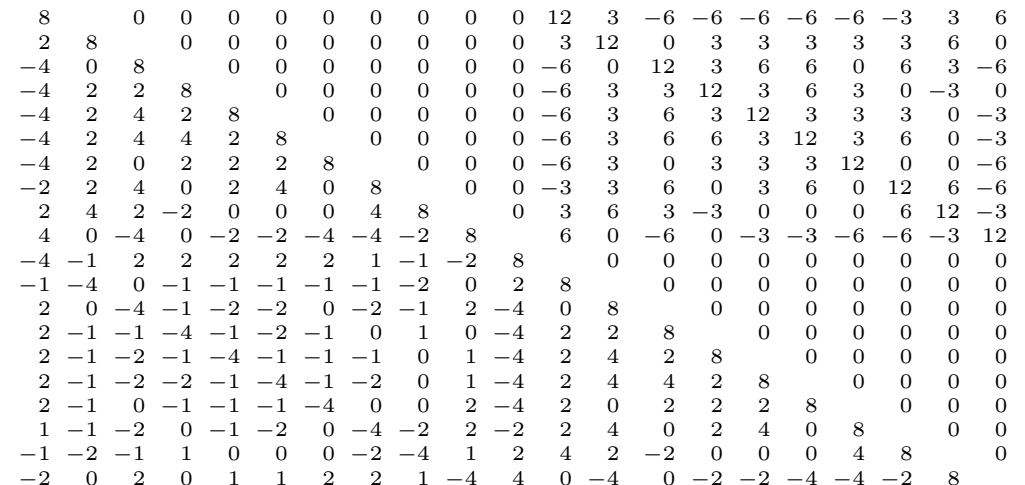

19: $\sqrt{-3}\left[C_{6}\right]_{1} \otimes A_{10}^{(3)}$

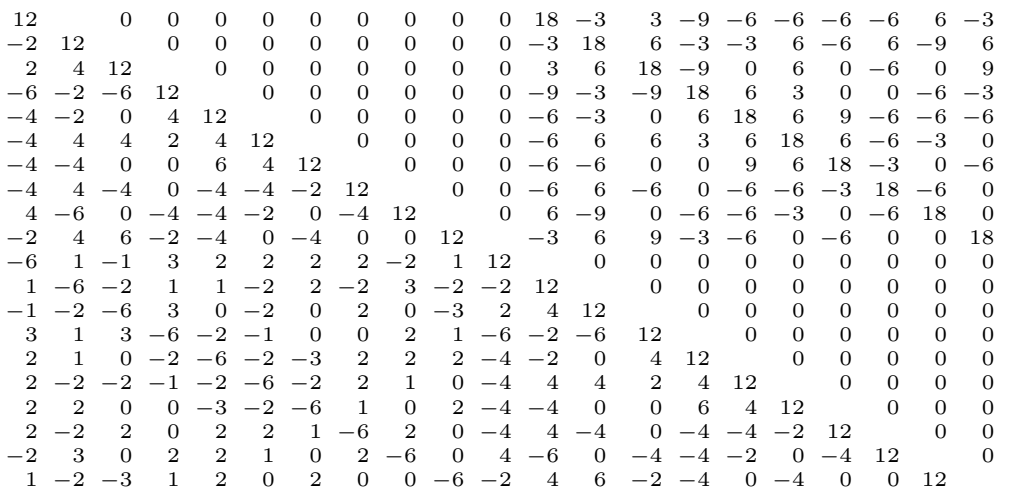

$$
\text { 20: } \infty, 2\left[\mathrm{SL}_{2}(11)\right]_{5} \circ C_{3}
$$

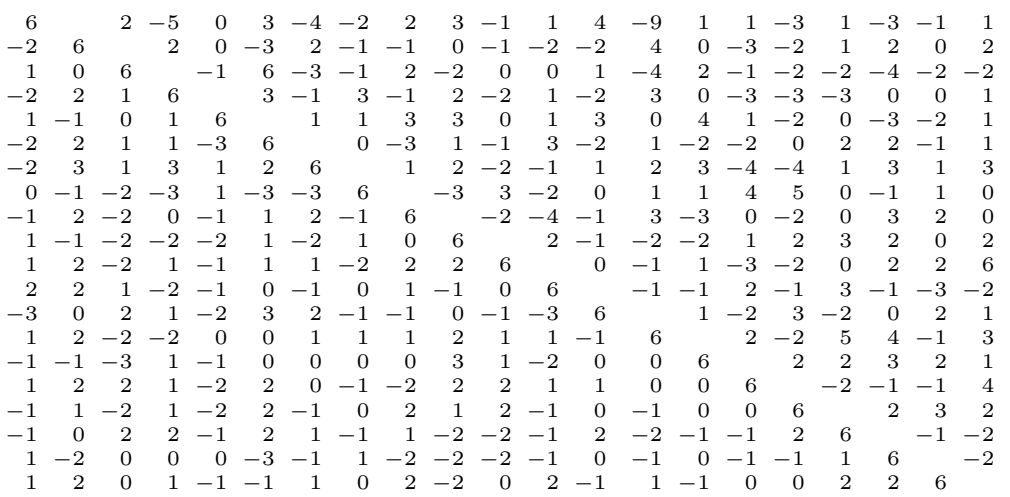


21: $\sqrt{-7}\left[2 . M_{22}: 2\right]_{10}$

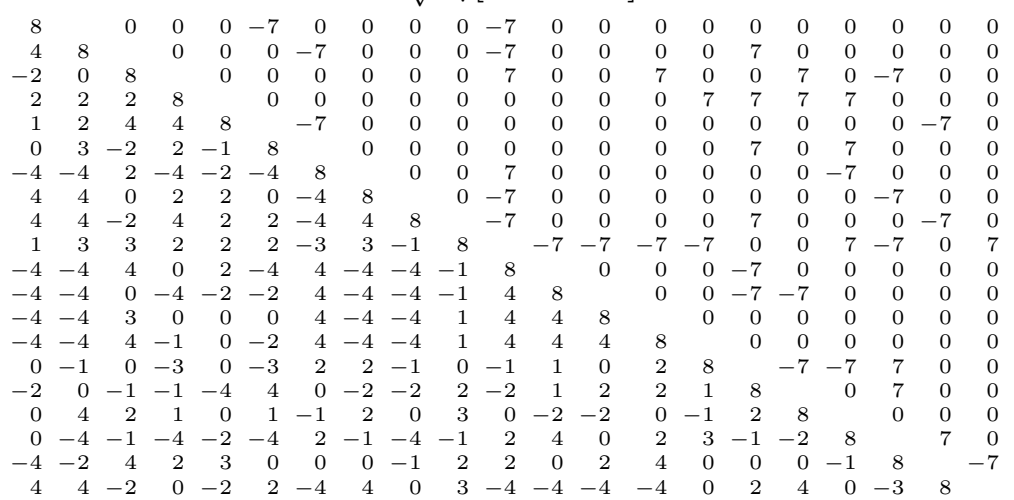

22: $\sqrt{-11}\left[ \pm \mathrm{L}_{2}(11)\right]_{5} \otimes \infty_{\infty, 2}\left[\mathrm{SL}_{2}(3)\right]_{1}$

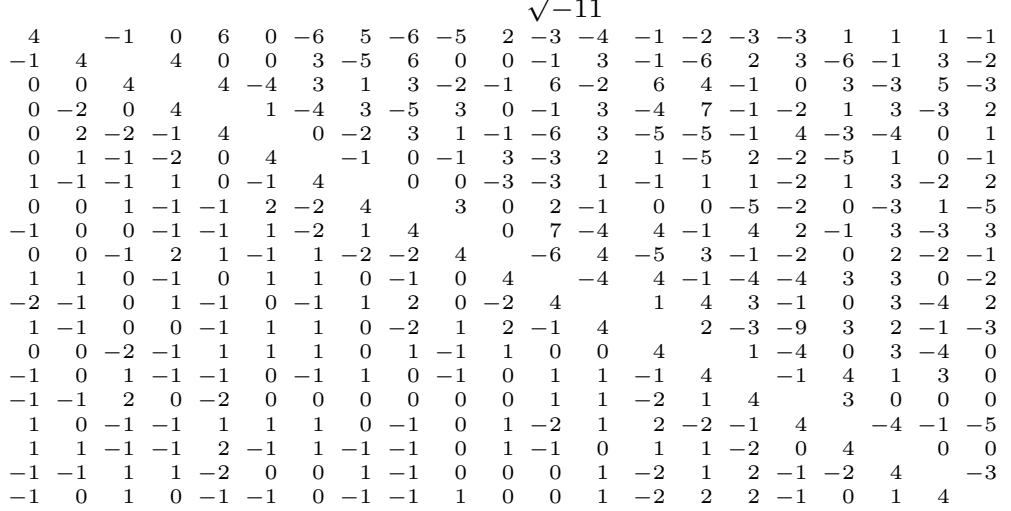

23: $\sqrt{-11}\left[ \pm \mathrm{L}_{2}(11)\right]_{5} \otimes A_{2}$

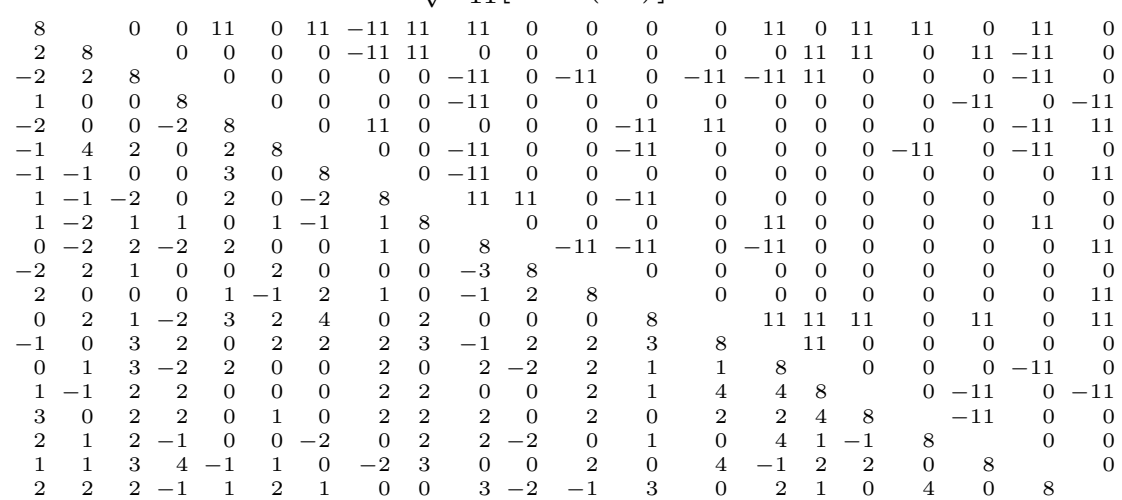

24: $\sqrt{-19}\left[\mathrm{SL}_{2}(19)\right]_{9}$

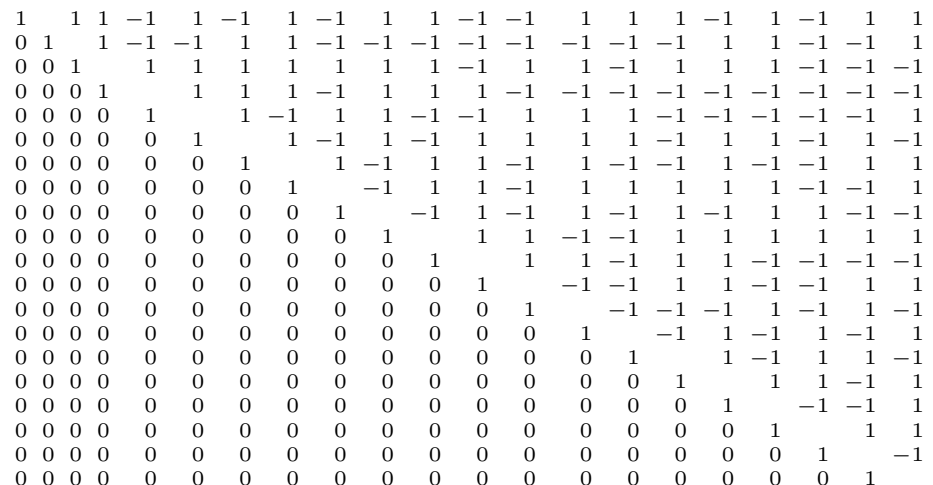


25: $\zeta_{10}\left[ \pm 5_{+}^{1+2} \cdot \operatorname{Sp}_{2}(5)\right]_{5}$

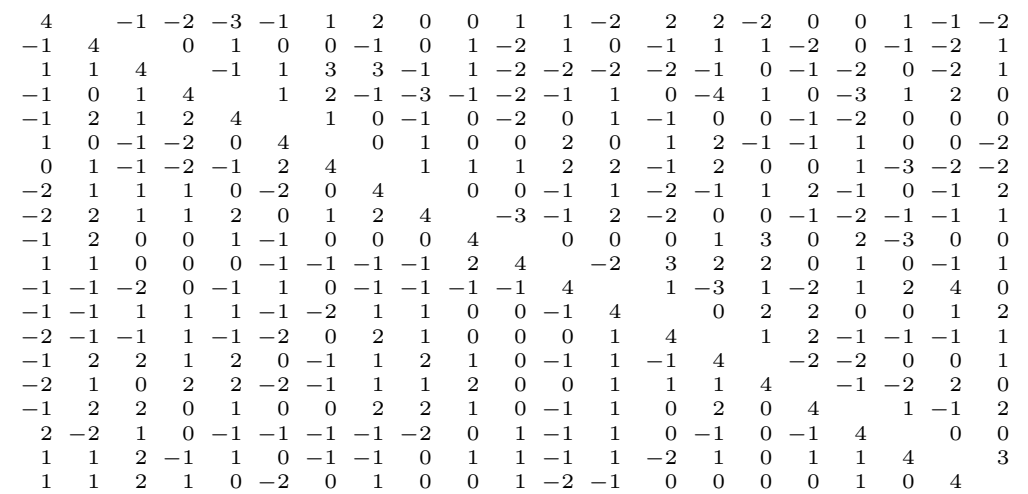

\section{A.11 Dimension 22}

1: ${ }_{i}\left[C_{4}\right]_{1} \otimes A_{11}$

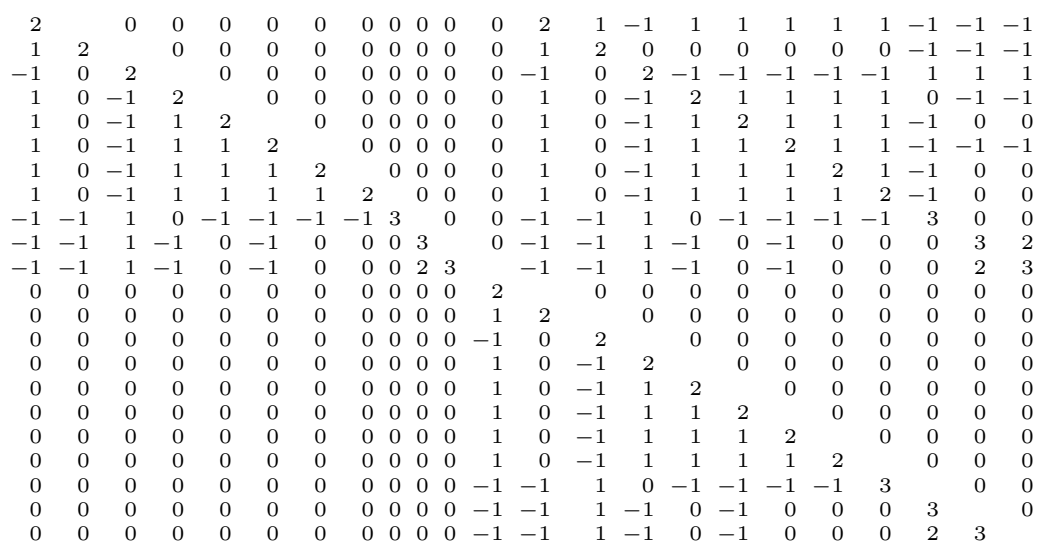

2: $\sqrt{-3}\left[C_{6}\right]_{1} \otimes A_{11}$

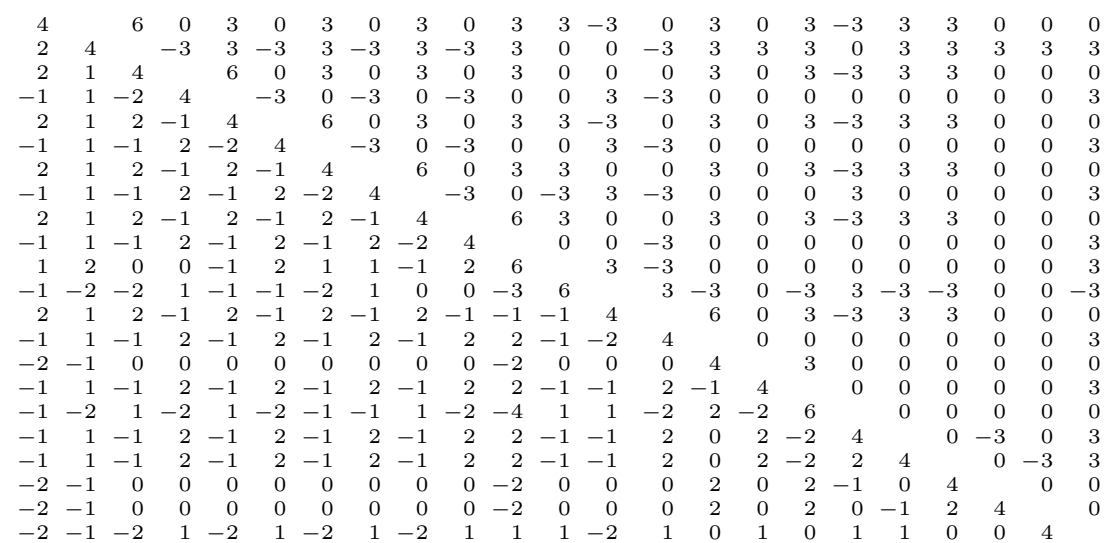


3: $\sqrt{-3}\left[ \pm U_{5}(2) \circ C_{3}\right]_{11}$

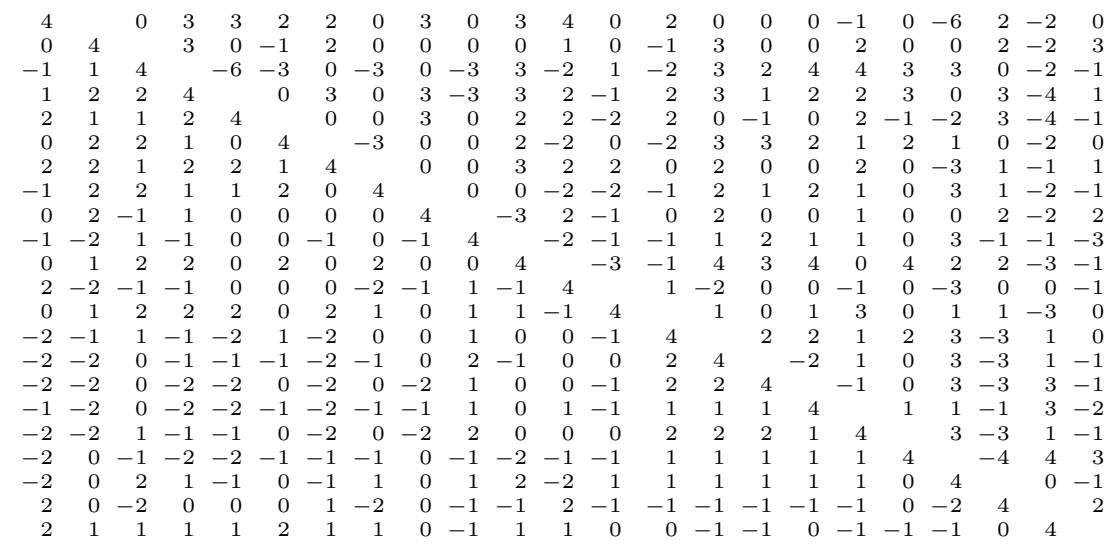

4: $\sqrt{-23}\left[ \pm \mathrm{L}_{2}(23)\right]_{11}$

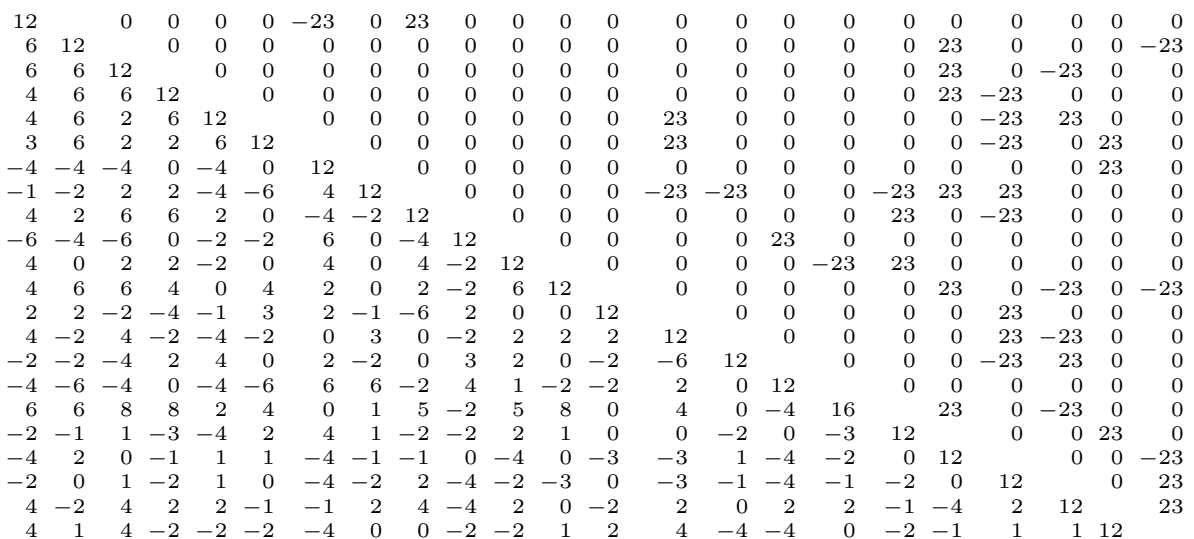




\section{Nomenclature}

Let $G<\mathrm{GL}_{m}(\mathbb{Q})$ be a matrix group and let $N$ and $H$ be any groups.

r.i.m.f. rational irreducible maximal finite

s.i.m.f. $\quad$ symplectic irreducible maximal finite

s.p.i.m.f. $\quad$ symplectic primitive irreducible maximal finite

$\Pi(k) \quad$ set of all primes dividing the integer $k$

$\tilde{\Pi}(K, k) \quad$ set of primes needed for the $m$-parameter argument cf. Definition 2.2 .10

$\mathbb{F}_{q} \quad$ finite field with $q$ elements

$\mathcal{Q}_{P_{1}, \ldots, P_{r}} \quad$ quaternion algebra with center $\mathbb{Q}$ ramified only at the places $P_{1}, \ldots, P_{r}$

$\mathcal{Q}_{\alpha, P_{1}, \ldots, P_{r}} \quad$ quaternion algebra with center $\mathbb{Q}(\alpha)$ ramified only at the places $P_{1}, \ldots, P_{r}$

$\mathbb{Z}_{K} \quad$ maximal order of an algebraic number field $K$

$\mathcal{C l}(R) \quad$ ideal class group of a Dedekind ring $R$

$\zeta_{n} \quad$ primitive $n$-th root of unity

$\theta_{n} \quad \zeta_{n}+\zeta_{n}^{-1}$

$I_{m} \quad m \times m$ identity matrix

$\mathcal{F}(G) \quad$ set of $G$-invariant forms cf. Definition 2.1.1

$\mathcal{F}_{\text {sym }}(G) \quad$ subset of symmetric $G$-invariant forms in $\mathcal{F}(G)$

$\mathcal{F}_{\text {sym }}(G) \quad$ subset of skewsymmetric $G$-invariant forms in $\mathcal{F}(G)$

$\mathcal{F}_{>0}(G) \quad$ subset of positive definite forms in $\mathcal{F}_{\text {sym }}(G)$

$\bar{G} \quad$ enveloping algebra of $G$ cf. Definition 2.1.1

$\operatorname{End}(\bar{G}) \quad$ endomorphism ring or commuting algebra $C_{\mathbb{Q}^{m \times m}}(G)$ of $G$ cf. Definition 2.1 .1

$\mathcal{Z}(\Lambda) \quad$ set of $\Lambda$-invariant lattices in $\mathbb{Q}^{1 \times m}$ for some $\mathbb{Z}$-order $\Lambda \subset \mathbb{Q}^{m \times m}$ cf. Definition 2.1 .4 
$\mathcal{Z}(G) \quad$ set of $G$-invariant lattices in $\mathbb{Q}^{1 \times m}$ cf. Definition 2.1.4

$\operatorname{det}(L, F) \quad$ determinant of the lattice $L$ in the Euclidean space $(L \otimes \mathbb{R}, F)$

$\operatorname{Aut}_{K}(L, \mathcal{F})$ group of $K$-linear automorphisms of the lattice $L$ wrt. the forms in $\mathcal{F}$ cf. Definition 2.1.4

$\mathcal{B}^{o}(G) \quad$ generalized Bravais group of $G$ cf. Definition 2.1.22

$\pm G \quad$ the group $\left\langle G,-I_{m}\right\rangle$

$O_{p}(H) \quad$ largest normal $p$-subgroup of $H$

$F(H) \quad$ Fitting subgroup of $H$, i.e. the largest normal nilpotent subgroup of $H$

$E(H) \quad$ layer of $H$, i.e. the central product of all components of $H$

$F^{*}(H) \quad$ generalized Fitting subgroup of $H$ (central product of $F(H)$ and $E(H)$ )

$A_{n}, F_{4}, E_{k}$ (automorphism groups of) root lattices

$C_{n} \quad$ cyclic group of order $n$

$S_{n}, \mathrm{Alt}_{n} \quad$ symmetric and alternating groups on $n$ letters

$D_{2 n} \quad$ dihedral group of order $2 n$

$Q D_{2^{n}} \quad$ quasidihedral group of order $2^{n}$

$Q_{2^{n}} \quad$ (generalized) quaternion group of order $2^{n}$

$2_{+}^{1+2 n} \quad$ central product of $n$ copies of $D_{8}$

$2_{-}^{1+2 n} \quad$ central product of $Q_{8}$ and $n-1$ copies of $D_{8}$

$p_{+}^{1+2 n} \quad$ extraspecial $p$-group of order $p^{1+2 n}$ and exponent $p$ ( $p$ odd prime)

$p_{-}^{1+2 n} \quad$ extraspecial $p$-group of order $p^{1+2 n}$ and exponent $p^{2}$ ( $p$ odd prime)

$G_{1} \otimes G_{2} \quad$ tensor product of the two matrix groups $G_{1}$ and $G_{2}$. See Section 2.4 for an explanation of the symbols $G_{1} \otimes G_{2}, G_{1} \underset{Q}{\otimes} G_{2}$ and $G_{1} \circ G_{2}$

$G_{1}^{2(p)} \otimes G_{2} \quad$ extension of $G_{1} \otimes G_{2}$ by $C_{2}$. See Section 2.4 for an explanation of the symbols $G_{1} \stackrel{2(p)}{\otimes} G_{2}, G_{1} \stackrel{2(p)}{\otimes} G_{2}, G_{1} \stackrel{2(p)}{\otimes} G_{2}, G_{1} \underset{Q}{\stackrel{2(p)}{\otimes}} G_{2}, G_{1} \stackrel{2(p)}{\otimes} G_{2}, G_{1} \underset{Q}{\stackrel{2(p)}{\otimes}} G_{2}, G_{1}^{2(p)} G_{2}$, $G_{1}^{2(p)} G_{2}$ and $G_{1} \stackrel{2(p)}{\square} G_{2}$

$N: H \quad$ semidirect product, i.e. a split extension of $N$ by $H$

$N \cdot H \quad$ nonsplit extension of $N$ by $H$

N.H any extension of $N$ by $H$

$H\left\ulcorner S_{k} \quad\right.$ wreath product of $H$ and $S_{k}$ 


\section{Bibliography}

[Art57] Emil Artin. Geometric Algebra, volume 3 of Interscience tracts in pure and applied mathematics. Interscience Publishers, Inc., 1957.

[Asc00] Michael Aschbacher. Finite Group Theory. Cambridge University Press, 2000 .

[BBNZ77] Harold Brown, Rolf Bülow, Joachim Neubüser, and Hans Zassenhaus. Crystallographic Groups of Four-Dimensional Space. Wiley, 1977.

[BCP97] Wieb Bosma, John Cannon, and Catherine Playoust. The Magma algebra system. I. The user language. J. Symbolic Comput., 24(3-4):235-265, 1997.

[Bli17] Hans F. Blichfeldt. Finite Collineation Groups. The University of Chicago Press, 1917.

$\left[\mathrm{CCN}^{+} 85\right]$ John H. Conway, Robert T. Curtis, Simon P. Norton, Richard A. Parker, and Robert A. Wilson. Atlas of finite groups. Clarendon Press, Oxford, 1985 .

[Dor71] Larry L. Dornhoff. Group Representation Theory. Pure and Applied Mathematics: A Series of Monographs and Textbooks. Marcel Dekker Inc., 1971.

[Fei76] Walter Feit. On finite linear groups in dimension at most 10. In Proceedings of the Conference on Finite Groups (Univ. Utah, Park City, Utah, 1975), pages 397-407, 1976.

[Fei82] Walter Feit. The representation theory of finite groups. North-Holland Publishing Co., 1982.

[GTT07] Meinolf Geck, Donna Testerman, and Jacques Thévenaz, editors. Group Representation Theory, chapter 6: Bounds of the Orders of Finite Subgroups of $\mathrm{G}(\mathrm{k})$. EPFL Press, 2007.

[HM01] Gerhard Hiss and Gunter Malle. Low-dimensional representations of quasisimple groups. LMS J. Comput. Math., 4:22-63, 2001. Corrigenda, same J., 5:95-126, 2002.

[Hup67] Bertram Huppert. Endliche Gruppen I. Springer, 1967.

[Isa94] I. Martin Isaacs. Character Theory of Finite Groups. Dover Publications, 1994. 
[Kne02] Martin Kneser. Quadratische Formen. Springer, 2002.

[KV] Markus Kirschmer and John Voight. Algorithmic enumeration of ideal classes for quaternion orders. submitted.

[Lor71] Falko Lorenz. Über die Berechnung der Schurschen Indizes von Charakteren endlicher Gruppen. J. Number Theory, 3:60-103, 1971.

[MGH98] Bernd H. Matzat, Gerd-Martin Greuel, and Gerhard Hiss, editors. Algorithmic Algebra and Number Theory, chapter The structure of maximal finite primitive matrix groups, pages 417-422. Springer, 1998.

[Min87] Hermann Minkowski. Zur Theorie der Positiven Quadratischen Formen. Journal f. d. reine und angewande Mathematik, 101:196-202, 1887.

[Neb95] Gabriele Nebe. Endliche rationale Matrixgruppen vom Grad 24. PhD thesis, RWTH Aachen University, 1995.

[Neb96] Gabriele Nebe. Finite subgroups of $\mathrm{GL}_{n}(\mathbb{Q})$ for $25 \leq n \leq 31$. Communications in Algebra, 24(7):2341-2397, 1996.

[Neb98] Gabriele Nebe. Finite quaternionic matrix groups. Representation Theory, 2:106-223, 1998.

[Nic06] Simon J. Nickerson. An Atlas of Characteristic Zero Representations. PhD thesis, University of Birmingham, 2006.

[NP95] Gabriele Nebe and Wilhelm Plesken. Finite rational matrix groups. In Memoirs of the American Mathematical Society, volume 116. AMS, 1995.

[NRS01] Gabriele Nebe, Eric M. Rains, and Neil J. A. Sloane. The invariants of the clifford groups. Designs, Codes and Cryptography, 24(1):99-122, 2001.

[Par84] Richard A. Parker. The computer calculation of modular characters (the meat-axe). In Computational group theory (Durham, 1982), pages 267-274. Academic Press, 1984.

[PH84] Wilhelm Plesken and Wilhelm Hanrath. The lattices of six-dimensional euclidean space. Mathematics of Computation, 43(168):573-587, 1984.

[Ple78] Wilhelm Plesken. On reducible and decomposable representations of orders. Journal für die reine und angewandte Mathematik, 297:188-210, 1978.

[Ple91] Wilhelm Plesken. Some applications of representation theory. Progress in Mathematics, 95:477-496, 1991.

[PS97] Wilhelm Plesken and Bernd Souvignier. Computing isometries of lattices. Journal of Symbolic Computation, 24:327-334, 1997.

[Rei03] Irving Reiner. Maximal Orders. Oxford Science Publications, 2003. 
[Sch05] Isaac Schur. Über eine Klasse von endlichen Gruppen linearer Substitutionen. Sitz. Preuss. Akad. Wiss., Berlin, pages 77-91, 1905. (Ges. Abh., Band I, nr. 6).

[Ste89] Leonid Stern. On the norm groups of global fields. Journal of Number Theory, 32(2):203-219, 1989.

[Wal62] Gordon E. Wall. On the Clifford Collineation, Transform and Similarity Groups (IV). Nagoya Mathematical Journal, 21:199-122, 1962.

[Was96] Lawrence C. Washington. Introduction to Cyclotomic Fields. Graduate Texts in Mathematics. Springer, 2nd edition, 1996.

[Win72] David L. Winter. The automorphism group of an extraspecial $p$-group. Rocky Mountain Journal of Mathematics, 2(2):159-168, 1972. 


\title{
Lebenslauf
}

\author{
Markus Kirschmer
}

27.04.1980 geboren in Giengen

1986 - 1990 Grundschule in Giengen (Bühlschule)

1990 - $1999 \quad$ Margarete-Steiff-Gymnasium in Giengen

$1999-2000 \quad$ Zivildienst in der IT-Abteilung des Rehabilitationskrankenhauses Ulm

Okt. 2000 - Mathematikstudium an der Universität Ulm

Sept. 2005

Aug. 2003 - Graduate studies in mathematics an der Syracuse University, NY, Mai $2004 \quad$ USA

Mai 2004 Master of Science, Syracuse University, NY, USA

Sep. 2005 Diplom in Mathematik von der Universität Ulm

Sep. 2005 - Stipendiat im Graduiertenkolleg "Hierarchie und Symmetrie in Aug. 2008 mathematischen Modellen" und Promotionsstudent an der RWTH Aachen

Sep. - Okt. Gastaufenthalt an der University of Sydney bei Prof. J. Cannon 2007

seit Okt. 2008 wissenschaftlicher Angestellter am Lehrstuhl D für Mathematik an der RWTH Aachen 